678 | August 2014

SCHRIFTENREIHE SCHIFFBAU

Bernadette Zipfel

Structural response of stranded ships

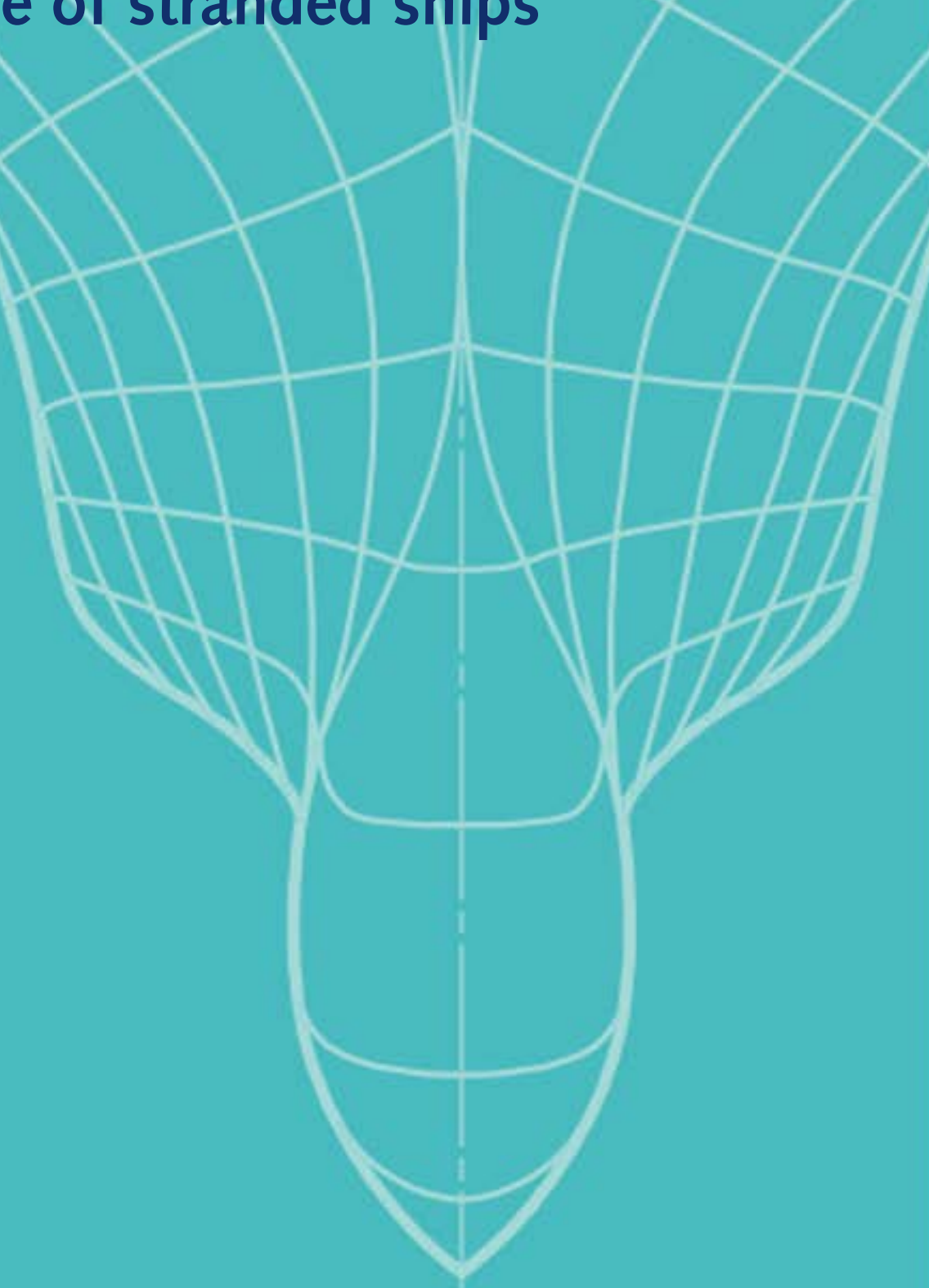





\title{
Structural response of stranded ships
}

\author{
Vom Promotionsausschuss der \\ Technischen Universität Hamburg-Harburg \\ zur Erlangung des akademischen Grades \\ Doktor-Ingenieurin (Dr.-Ing.) \\ genehmigte Dissertation
}

von
Bernadette Zipfel

aus

Berlin

2014 


\section{Gutachter}

1. Gutachter: Prof. Dr.-Ing. Dr.-Ing. E.h. Dr. h.c. Eike Lehmann

2. Gutachter: Prof. Dr.-Ing. Jürgen Grabe

\section{Tag der mündlichen Prüfung}

14. Juli 2014

(c) Schriftenreihe Schiffbau der

Technischen Universität Hamburg-Harburg

Schwarzenbergstraße 95c

D-21073 Hamburg

http://www.tuhh.de/vss

Bericht Nr. 678

ISBN 978-3-89220-678-1

URN: urn:nbn:de:gbv:830-tubdok-12838 


\section{Exclusive summary}

The thesis reports on different methods developed to assess the structural response of ships to soft grounding accidents, also referred to as stranding. These are exemplarily applied to a container vessel. During stranding a vessel comes to rest upon a soft seabed and can be subjected to the tide. If the water recedes, the hydrostatic forces change significantly and the ultimate capacity is reduced. Severe global damage can be the consequence. The ability to predict accidents and assess their consequences in order to minimise damage is of great interest. This work contributes to the design of vessels against accidental load.

A simple method based on the ideal beam theory is presented to calculate the critical combinations of stranding point and area, tidal range and load case, which lead to an exceedance of the permissible bending moment and shear force and/or to instability, as well as the additional moments and forces during stranding. The ship's form is not simplified. Of all calculations stranding incidents amidship result in the highest bending moments and shear forces. The shear force is detrimental at the vessels ends. Building on those results a simulation procedure using the finite element method is developed to realistically simulate the damage process of the structure. The comparison with a real stranding accident shows very good conformance. The advantage of the method is that the local and global damage to the structure can be examined as a function of the soil characteristics, ground geometry, the position of stranding and ebb tide. Most calculated scenarios end in global damage to the structure and a fractured outer hull. The degree of damage, the damage mode and the ultimate strength depend on the ground geometry and the soil characteristics. It is necessary to model the ground with a sand material model because the hull girder is not always less prone to hull collapse if set onto a soft bank. Furthermore, the degree of collapse cannot be concluded from the height of the external forces and moments. The bending moments and shear forces have a different influence not only on the damage but also on the hull capacity. Equations to assess moment-shear force interaction curves based on the true and combined loading conditions during stranding are developed to evaluate the influence of the shear force on the ultimate hull capacity. The true loading of the structure during stranding reduces the ultimate strength by $10 \%$.

The proposed methods are applicable to all ship types and ground geometries and to many soil characteristics.

In der Dissertation werden verschiedene Methoden vorgestellt, um die Strukturantwort von Schiffen bei weicher Grundberührung, auch Strandung genannt, zu berechnen. Sie werden exemplarisch an einem Containerschiff gezeigt. Bei Strandung liegt das Schiff auf weichem Boden auf und kann dann Tideeffekten ausgesetzt sein. Wenn das Wasser abläuft, ändern sich die hydrostatischen Kräfte und die Grenztragfähigkeit der Struktur wird überschritten. Es können starke Schäden auftreten bis hin zum Durchbrechen des gesamten Schiffes. Es ist von großem Interesse, diese Unfälle berechnen zu können, um den Schaden zu minimieren. Diese Arbeit stellt Methoden vor, um Strukturen gegen globale Schäden zu entwerfen.

Eine einfache Methode, basierend auf der idealen Balkentheorie wird eingeführt, mit der die kritischen Kombinationen aus Strandungsposition, Tideeffekten und Beladung, die zum Überschreiten des zulässigen Biegemomentes und der zulässigen Querkraft oder Instabilität führen, berechnet werden können. Die Schiffsform wird nicht vereinfacht. Von allen berechneten Strandungsfällen ergeben sich die größten Momente und Querkräfte bei 
Strandung im Mittschiffsbereich. Die Querkraft ist im Falle der Strandung im Bereich der Schiffsenden kritisch. Darauf aufbauend wird ein Verfahren unter Verwendung der Finiten-Elemente Methode entwickelt, um lokale und globale Schäden bei Berücksichtigung aller realen Effekte zu berechnen. Der Vergleich mit einem bekannten Strandungsfall zeigt gute Übereinstimmungen. Der Vorteil der Methode ist, dass der lokale und globale Schaden in Abhängigkeit der Bodeneigenschaften und -geometrie, Strandungspositionen und bei ablaufendem Wassers bestimmt werden kann. Fast alle berechneten Strandungsszenarien führen zum globalen Schaden und zum Aufreißen der Außenhaut. Die Schadensintensität, Schadensart und die Traglast hängen von der Bodengeometrie sowie den Bodeneigenschaften ab. Der Boden sollte mit einem Sandmaterial simuliert werden, weil der Strukturschaden bei Strandung auf weichem Untergrund größer sein kann als bei Strandung auf (vereinfachtem) hartem Boden. Des Weiteren kann die Schadensintensität nicht anhand der externen Momente und Querkräfte abgeschätzt werden. Die Biegemomente und Querkräfte haben einen unterschiedlichen Einfluss nicht nur auf den Schaden, sondern auch auf die Tragfähigkeit. Formeln zur Berechnung von Momenten-QuerkraftInteraktionskurven werden entwickelt um u.a. den Einfluss der Querkraft auf das Traglastmoment bei Strandung abzuschätzen. Die tatsächliche Belastung während der Strandung führt zu einer Reduzierung der Traglast um 10\%.

Die vorgeschlagenen Methoden sind auf alle Schiffstypen, Bodengeometrien und -eigenschaften anwendbar. 


\section{Danksagung}

Ich danke ganz besonders Herrn Prof. Dr.-Ing. Dr.-Ing. E.h. Dr. h.c. Eike Lehmann für die Betreuung meiner Doktorarbeit. Die intensive Zusammenarbeit, seine Erfahrung, Anregungen und Ideen sowie viele fruchtbare Diskussionen haben diese Arbeit ermöglicht. Außerdem danke ich Herrn Prof. Dr.-Ing. Jürgen Grabe für die Begutachtung meiner Arbeit.

Ich danke der Deutschen Forschungsgesellschaft (DFG) für die Finanzierung des Projekts. Diese Arbeit ist Teil des Graduiertenkollegs ,,Seehäfen für Containerschiffe zukünftiger Generationen: Interaktion von Schiff, Fluid, Struktur und Boden“ (GRK1096) an der Technischen Universität Hamburg-Harburg.

Bei meinen Kollegen am Institute für Festigkeit und Konstruktion von Schiffen und im Graduiertenkolleg an der Technischen Universität Hamburg-Harburg möchte ich mich ebenfalls bedanken. Stellvertretend danke ich Herrn Dipl.-Ing. Martin Schötteldreyer für die die ausgesprochen nette und hilfsbereite Atmosphäre. Auch die Studierenden Ingo Voigt, Jasmin Grigat, Sören Schenke, Christoph Meier, Kai-Nicolas Steimer und Tim Janele, deren Bachelor-, Master-, Projekt-, Studien- und Diplomarbeiten ich betreuen durfte, haben mit ihren Untersuchungen und Modellerstellungen zum erfolgreichen Entstehen dieser Arbeit beigetragen.

Nicht zuletzt gilt mein Dank meinem Mann Jochen, der mir, wenn nötig, den Rücken freigehalten, gegen aufflackerende Zweifel gestärkt und viele Texte gegengelesen hat. Besonders dankbar bin ich meinen Kindern Paula und Luise für ihre Geduld und Fröhlichkeit während meiner wissenschaftlichen Arbeit. 



\section{Contents}

Nomenclature $\quad$ v

$\begin{array}{ll}\text { 1. Introduction } & 1\end{array}$

2. State-of-the-art of science and technology 5

2.1. Stranding of ships . . . . . . . . . . . . . . . 5

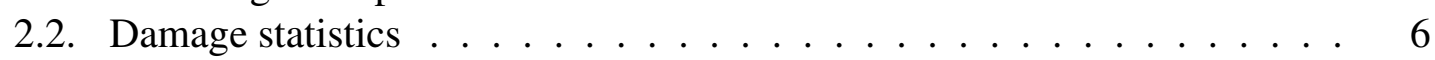

2.3. Approaches for stranding accidents . . . . . . . . . . . . . . 7

2.4. Ultimate hull girder strength . . . . . . . . . . . . . . . . . . . 13

2.4.1. Ultimate hull girder strength check in classification rules . . . . . 14

2.4.2. Interaction of ultimate bending moment and shear force . . . . . 21

2.5. Finite element method . . . . . . . . . . . . . . 27

3. Parameter study 31

3.1. The proposed method . . . . . . . . . . . . . . 31

3.1.1. Grounding calculation in $e 4 \ldots \ldots 31$

3.1.2. Simple model . . . . . . . . . . . . . . . . 32

3.1.3. Dimensions and load cases ...................... 33

3.1.4. Optimisation method . . . . . . . . . . . . . . . . . . 34

3.2. Verification of the simple method . . . . . . . . . . . . . . 39

3.3. Calculation results . . . . . . . . . . . . . . . . 42

3.3.1. Stranding case A1 . . . . . . . . . . . . . . 42

3.3.2. Stranding case A2 . . . . . . . . . . . . . . . . . 47

3.3.3. Stranding case A1y . . . . . . . . . . . . . . . . . . . 49

3.3.4. Stranding cases B1 and $\mathrm{C} 1 \ldots \ldots \ldots 50$

3.3.5. Stranding case A1f . . . . . . . . . . . . . . . 50

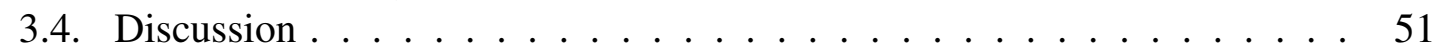

3.5. Conclusion ........................... 51

4. Simulation of stranding scenarios $\quad 53$

4.1. Description of simulation method ... . . . . . . . . . 53

4.1.1. Ship model ....................... 55

4.1.2. Ground models . . . . . . . . . . . . . . . . . 58

4.2. Verification of the simulation method . . . . . . . . . . . . . 62

4.2.1. Ship model . . . . . . . . . . . . . . . . . . . . . 62

4.2.2. Soft ground model . . . . . . . . . . . . . . . . . . 64

4.3. Specification of calculation parameters . . . . . . . . . . . . . . 66

4.3.1. Load increase . . . . . . . . . . . . . . . . . . . 67

4.3.2. Time step size . . . . . . . . . . . . . . 67 


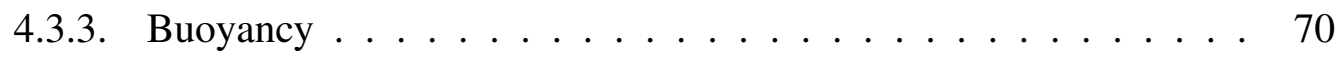

4.3.4. Element type and failure criterion . . . . . . . . . . . . . . 70

4.3.5. Summary of calculation parameters . . . . . . . . . . . . 72

4.4. Comparison with double T-beam . . . . . . . . . . . . . . . 72

4.5. Results of the midship scenario . . . . . . . . . . . . . . . . . . 74

4.5.1. Bending moment and shear force curves . . . . . . . . . . . 75

4.5.2. Bending moment $\mathrm{M}_{\mathbf{h}} \ldots \ldots \ldots \ldots 77$

4.5.3. Stress and collapse . . . . . . . . . . . . . . . . . . . . . . . . . . . . . 79

4.5.4. Influence of ground . . . . . . . . . . . . . . . 84

4.6. Analysing the Fowairet stranding incident . . . . . . . . . . . . . . . . . . . 86

4.6.1. Specifics of the Fowairet . . . . . . . . . . . . . . . . . . . . . . . . . . 87

4.6.2. Specifics of the incident . . . . . . . . . . . . . . 87

4.6.3. Comparing the damage of the Fowairet to Sa7p2 . . . . . . . . . 88

4.7. Results of the bow and the stern scenarios . . . . . . . . . . . . . 91

4.7.1. Stranding at the bow section . . . . . . . . . . . . . . . . . . . . . . 91

4.7.2. Stranding at the stern section . . . . . . . . . . . . . . . 94

4.8. Relation of moment and shear force in the midship, bow and stern scenarios 96

4.9. Discussion . . . . . . . . . . . . . . . . . . 97

4.10. Conclusion . . . . . . . . . . . . . . . . 98

5. Ultimate load calculation 101

5.1. Recalculation of box girder experiments . . . . . . . . . . . . 101

5.1.1. Model 31, 22 and 23 under pure bending moment . . . . . . . . . 102

5.2. Moment-shear force interaction of box girders . . . . . . . . . . . . . . 107

5.3. Moment-shear force interaction of simplified cross sections . . . . . . . . 110

5.4. Comparing UHGS of simplified sections, stranding scenario and classifi-

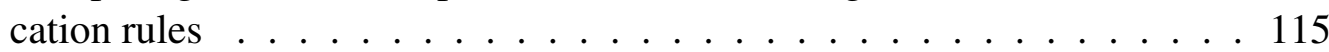

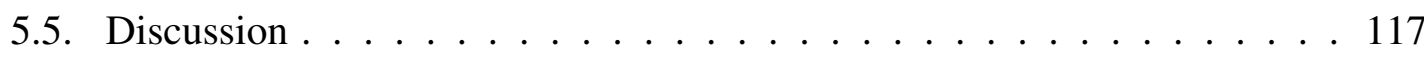

5.6. Conclusion ......................... 118

$\begin{array}{ll}\text { 6. Conclusions } & 119\end{array}$

$\begin{array}{ll}\text { Bibliography } & 123\end{array}$

A. Parameter Study 129

A.1. Optimisation method . . . . . . . . . . . . . . . . . . . . . . 129

A.2. Stranding case B1 and C1 . . . . . . . . . . . . . . . . . 133

A.3. Stranding case A1f . . . . . . . . . . . . . . . 136

B. Simulation of stranding scenarios $\quad 137$

B.1. Moment of inertia . . . . . . . . . . . . . . . . . . . . . . . 137

B.2. Construction drawings . . . . . . . . . . . . . . . . . 138

B.3. Oedometer test . . . . . . . . . . . . . . . . . . . . . . . 139

B.4. Shear force of Ha7p2 and Sa7p2 at 146, 147 and 148 m AP . . . . . . . . 140

B.5. Influence of ground . . . . . . . . . . . . . . . . . . 141

B.5.1. Ground parameters . . . . . . . . . . . . . . . 141

B.5.2. Ground geometries . . . . . . . . . . . . . . . 141 
B.6. Results of bow and stern scenarios . . . . . . . . . . . . . . . . 143

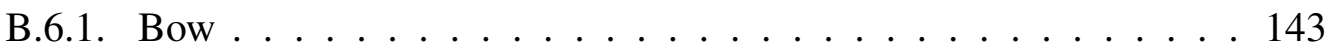

B.6.2. Stern ......................... 144

C. Ultimate load calculation $\quad \mathbf{1 4 5}$

C.1. Cross sections . . . . . . . . . . . . . . . . . . . . . . . . 145

C.2. Moment-shear force interaction of bow and stern sections . . . . . . . . 147 



\section{Nomenclature}

\section{General}

$\begin{array}{ll}\nabla & \text { displaced volume of ship } \\ \sigma_{F} & \text { yield stress } \\ \tau_{p l} & \text { plastic shear stress } \\ \text { A } & \text { cross section area } \\ \text { AP } & \text { aft perpendicular } \\ A_{s} & \text { effective shear area } \\ A_{z} & \text { waterplane area } \\ B & \text { breadth of ship } \\ c_{B} & \text { block coefficient } \\ c_{M} & \text { mainframe coefficient } \\ c_{W p} & \text { waterplane coefficient } \\ D & \text { height of ship } \\ \text { e.n.a } & \text { elastic neutral axis } \\ \text { FP } & \text { forward perpendicular } \\ g & \text { acceleration of gravity } \\ G M_{L} & \text { longitudinal metacentric height } \\ k & \text { discrete field coordinate for } x_{c o n t} \\ L_{p p} & \text { length of ship between perpendicular } \\ m & \text { discrete field coordinate } s_{d} \\ M_{p l} & \text { plastic limit moment } \\ M_{T} & \text { class permissible bending moment }=M_{S W}+M_{W V} \\ M_{S W} & \text { class permissible still water bending moment } \\ M_{W V} & \text { class permissible vertical wave bending moment } \\ n & \text { discrete field coordinate for } x_{f A P} \\ \text { p.n.a } & \text { plastic neutral axis } \\ Q_{p l} & \text { plastic limit shear force } \\ Q_{T} & \text { class permissible shear force }=Q_{S W}+Q_{W V} \\ Q_{S W} & \text { class permissible still water shear force } \\ Q_{W V} & \text { class permissible vertical wave shear force } \\ T_{A P} & \text { draught at aft perpendicular } \\ \text { UHGS } & \text { ultimate hull girder strength } \\ W_{e l} & \text { elastic section modulus } \\ W_{p l} & \text { plastic section modulus } \\ s_{d} & \text { receding tide level } \\ x_{c o n t} & \text { stranding point } \\ x_{f A P} & \text { ship length coordinate } \\ & \end{array}$




\section{Abbreviation of FE calculations}

$\begin{array}{ll}\text { case A1 } & \text { beam calculation } \\ \text { Ha7p2 } & \text { hart ground }(\mathrm{H}), \text { slope angle } 7^{\circ} \text { (a7), plateau length } 2 \mathrm{~m}(\mathrm{p} 2) \\ \text { Ha7p2 bow } & \text { identical to Ha7p2 for stranding at bow part } \\ \text { Ha7p2 stern } & \text { identical to Ha7p2 for stranding at stern part } \\ \text { Sa7p2 } & \text { soft ground }(\mathrm{S}), \text { slope angle } 7^{\circ}(\mathrm{a} 7) \text {, plateau length } 2 \mathrm{~m}(\mathrm{p} 2) \\ \text { Sa7p2 bow } & \text { identical to Sa7p2 for stranding at bow part } \\ \text { Sa7p2 stern } & \text { identical to Sa7p2 for stranding at stern part } \\ \text { Sa7p22 } & \text { soft ground, slope angle } 7^{\circ} \text {, plateau length } 22 \mathrm{~m} \\ \text { Sa7p22w10 } & \text { soft ground, slope angle } 7^{\circ}, \text { plateau length } 22 \mathrm{~m} \text {, width } 10 \mathrm{~m} \\ \text { Sa15p2 } & \text { soft ground, slope angle } 15^{\circ}, \text { plateau length } 2 \mathrm{~m} \\ \text { Sa15p2 bow } & \text { identical to Sa15p2 for stranding at bow part } \\ \text { Sa15p2 stern } & \text { identical to Sa15p2 for stranding at stern part } \\ \text { SGa7p2 } & \text { Sa7p2 with material model of sandbank } \\ \text { tbeam } & \text { T-beam geometry; material, loads, boundaries equal to e16f2 }\end{array}$

\begin{tabular}{ll} 
variations of Ha7p2 & differences to Ha7p2 \\
\hline e16f1 & element type (tab. 4.8) \\
e16f2 & failure criterion, element type 16 (tab. 4.8) \\
e16f3 & failure criterion (tab. 4.8) \\
li5 & 5 s of load increase (tab. 4.5) \\
li15 & 15 s of load increase (tab. 4.5) \\
li30 & 30 s of load increase (tab. 4.5) \\
Ha7p2B & loads due to buoyancy \\
Ha7p2el & ideal elastic material, element type 16 \\
t1a1 & area of beam elements, time step size (tab. 4.6) \\
t1a1d & area of beam elements, time step size, damping (tab. 4.6) \\
t1a2 & time step size (tab. 4.6)
\end{tabular}




\section{Introduction}

During stranding or soft grounding accidents a vessel comes to rest upon a seabed. Such incidents are more likely to happen today than in the past in spite of continuous efforts to prevent them. They can lead to loss of human life, severe environmental consequences and economic losses. With the increasing demand for safety at sea and protection of the ecosystem, the ability to predict accidents, assess their consequences and minimise the damage of an accident is of great interest.

The probability of stranding incidents occurring, especially near harbours, is on the increase for several reasons. Ship dimensions are still growing, harbour depth is more and more limited and there is less and less room to manoeuvre.

The movement of goods is rising and more cost-efficient means of transportation are still needed. A possible solution to the problem is to use larger ships. As a result, ship dimensions are continuously growing and plans to build vessels with a capacity of 22000 TEU have already been announced, (United Nations [77]). At the same time the enlargement of existing ports, especially in Europe, lags behind the commissioning of new generations of ships. A good example is the Port of Hamburg, the biggest port in Germany. The ninth deepening of the river Elbe has been planed since 2002. The dredging has not yet begun but the ships for which the enlargement was planned are already sailing. Ships with a draught of $13.5 \mathrm{~m}$ can reach the Port of Hamburg without tidal effects. Those with a maximal draught of $14.5 \mathrm{~m}$ must use tidal effects. At the moment vessels with a maximum draught of $13.5 \mathrm{~m}$ call at the Port of Hamburg during the flood tide. The currently largest container vessels ("Triple E" class) have a maximum draught of $15.5 \mathrm{~m}$.

In addition to the limited depth of harbours, the demurrage is reduced and consequently the manoeuvring room decreases.

Alongside the increasing probability of grounding accidents, the new generation of container ship poses various challenges for harbour construction, e.g. container cranes and handling facilities, quay construction, manoeuvrability of large vessels, erosions and scours of the harbour bottom. The German Research Foundation (DFG) established the Research Training Group "Ports for Container Ships of Future Generations: Interaction of Ship, Fluid, Structure and Soil" (GRK 1096) to promote basic research to meet those challenges. The present work forms part of the group's work.

Research on the field of collision and grounding has begun early. First, the collision of two ships was considered. Later, detailed studies on the punctuation of the hull structure by diverse hard indenters were performed. There is a tendency in research to concentrate on grounding on a sharp rock as a worst case scenario but the likelihood of such an occurrence is small. Research with focus on groundings on soft underground (also called strandings) such as sand, silt or clay is rare. During stranding, the energy dissipation of 
the structure is low. A punctuation of the outer hull structure does not occur. The ship's speed is reduced and if there is enough friction it is stopped. When the contact area is too large, additional measures have to be taken to drag the vessel free. A problem is that stranded ships can be subjected to the tide. If the water level drops, the hydrostatic forces change significantly and the structure can sustain a severe damage. In the worst case the vessel breaks into two. But the crew has time until the next flood tide to take the right action to save the ship.

Oil spills are the most typical consequence of grounding. A recent example is the stranding of the container vessel Fowairet in September 2005. The vessel, which has a capacity of 3800 TEU, was on its way to the Port of Antwerp (Belgium). Loaded with 1952 TEU, it grounded in the Western Scheldt (The Netherlands) one hour after high tide. There was no immediate damage to the vessel, but it started to crack as the tide receded, resulting in oil leakage. A total rupture could be prevented. The salvage operation took two days. During the third flood tide after the accident, the vessel was re-floated.

This event demonstrates that the risk level is not acceptable. Improvement of accident prevention measures is essential to obtain the desired level of safety and protection of the ecosystems. At the moment, very few tools are available to assess the consequences of stranding. There are no methods in practice or in research to calculate stranding in all details. Therefore, it is important to develop simple methods to estimate the additional forces and moments on the ship structure resulting from a stranding incident. Furthermore, for a performance-based ship design, theoretical tools should be available to determine the damage and the consequences of the accident with limited modelling and calculation effort.

Under normal conditions, the shear force needs to be very high to have an influence on the bending moment. But during grounding the following aspects change the influence of the shear force:

- The maximum shear force due to grounding is at the same position as the maximal change of the bending moment. The highest values of shear force and bending moment can coincide.

- Ships have a relatively low form factor $\alpha=W_{p l} / W_{e l}$, because their cross sections are thin walled and often open. So their cross section capacity is small.

All calculations of this work are exemplarily applied to container vessels. The dimensions of container ships are growing very fast. Especially container vessels are at risk of sustaining severe damage during stranding. They have a low plastic reserve because of their thin walled and open cross sections. Load forces are introduced into the bottom structure at the bulkheads. If the ship comes to rest on a sandbank that is positioned in the middle of a cargo hold there are no forces from the cargo on the inner double bottom.

Furthermore, container vessels are slender ships. The effect of trimming around the contact area is greater than for full-bodied ships, since bow and stern have a very different shape. 
The stranding process can be divided into two stages. The first stage begins with the initial contact, includes the sliding phase and ends in the final stranding position. The vessel does not suffer important damage during this stage (see section 2 and 3). The process can be simulated independently with the kinetic model of Simonsen and Wierzbicki [73] and Simonsen [69]. In the second stage the vessel is subjected to tidal actions. The structural damage and the integrity of the stranded vessel has to be solved. This second stage is investigated in the present work. 



\section{State-of-the-art of science and technology}

First the principles of stranding are described. Then damage statistics are given to demonstrate the importance of the research field and to help identify realistic accident scenarios. Later, existing theoretical and numerical approaches for grounding are discussed. Approaches for the determination of the ultimate hull girder strength follow, because this is the criterion used to dimension the structure against extreme loads.

\subsection{Stranding of ships}

Ship stranding is a very complex process. Large contact forces, crushing of hull structure and interaction with global motions result in a highly nonlinear process.

The vessel sails between cruising and manoeuvring speed in tidal waters. It runs on a soft shoal. The ship structure does not suffer important damage between the initial contact with the ground and the final stranding position. The impulse of the contact is basically inelastic and reduces the ship's speed. If there is enough energy the ship is stopped and then lies on the ground. The soft ground will not penetrate the hull structure and the energy dissipation of the structure is low.

If the vessel is lying on the ground and the contact area is too large, additional measures have to be taken to drag the vessel free. This has to happen immediately, otherwise the stranded ship can be subjected to the receding tide and will loose substantial buoyancy. The new distribution of the hydrostatic forces depends on the ship's form, the draught, the trim and the heel. If the contact to the ground does not exactly level with the ship's centre of gravity and the surface is drawn down, the ship's draught changes and it trims and/or heels around the contact point. The hydrostatic pressure forces are redistributed. The ship partly loses its buoyancy. The lost buoyancy forces are introduced into the hull structure at the grounding area via bedding pressure. The maximum shear force is now at the same position as the maximal change of the bending moment. The interaction of the contact force with the hogging bending moment affects the longitudinal resistance of the hull. The predominant kind of damage is elasto-plastic buckles. If the ultimate hull girder strength is reached, global damage can occur. As a result, bow and stern could immerse and rotate around the contact area. This effect may reduce the hogging moment.

Not only the structure but also the soft ground is of deformable material. The load carrying capacity of the soil depends on its properties and conditions. "Soft ground" can consist of different soil types, such as sand, silt, gravel or clay (among others). Compactness and patterns in the arrangement of the particles as well as the void ratio and pore fluid distributions define the capacity. Therefore, the contact forces between soil and structure 
also depend on the soil stress-stain behaviour.

\subsection{Damage statistics}

Data concerning the frequency and scenarios of stranding accidents are rare. In fact, most classification societies have their own damage statistics. But the data are usually not released out for publication. Open source statistics are given by some countries of registration. The Federal Bureau of Maritime Casualty Investigation (BSU) of Germany lists all accidents involving professional vessels that occur in German waters and those involving vessels under the German flag. But the statistics are sorted differently according to the requirements of the respective societies. They all collect different information on accidents. Furthermore, shipowners are not interested in reporting accident information. They are typically afraid that the occurrence of an accident could have a negative effect on their reputation. So most likely some accidents are not disclosed and some reports underestimate the extent of damage. Statistics are based on past events and they may not reflect the present situation. In damage statistics only the final damage is given. It is not immediately possible to derive the accident scenario, in particular the shape of the ground. However, some statistics are given in table 2.1 to give an overview.

Table 2.1.: Grounding statisics.

\begin{tabular}{lrrr}
\hline source & no. of accidents & groundings & years \\
\hline Hampel [23] & 3500 & $14 \%$ & $1990-1996$ \\
$G L$ & 1356 & $40 \%$ & $2000-2009$ \\
Lützen [40] & 2946 & $32 \%$ & $1950-2003$ \\
Samuelides [65] & 441 & $43 \%$ & $1993-2002$ \\
$B S U$ & 679 & $14 \%$ & $2004-2009$ \\
\hline
\end{tabular}

Hampel [23] analysed data by the classification society Germanischer Lloyd (GL) for vessel with a gross tonnage (GRT) over 100. Of the registered accidents $43 \%$ were groundings on granular soil and $57 \%$ on rocks. In $51 \%$ of the grounding accidents the place of junction was in the area of the cargo holds. 25\% happened in the aft part and $24 \%$ in the fore part of the ship.

Lützen and Simonsen [40] analysed the HARDER (a project financed by the European Union) damage database, which is collected from seven different sources. The vessels are mostly conventional cargo ships with a length between 16 and $350 \mathrm{~m}$. In $80 \%$ of the grounding incidents the damage was below the tank top of the double bottom. Most vessels were sailing at a reduced speed before the accident and the water depth was near the draught of the vessel. The location of the centre of damage was mainly forward of the midship.

Samuelides et al. [65] presented statistics for vessels over 100 GRT sailing under the Greek flag. Assuming that the ship's life is 25 years, Samuelides et al. [65] concluded 
that every fourth ship has a grounding during its lifetime.

The reviewed statistics give very different frequencies of grounding incidents. Other publications, which are summarised in Committee III.1 [9] and Committee V.1 [10] of the ISSC, do not provide more clarity. It is not possible to determine how many vessels ground during their lifetime. However around one third of the reported accidents seem to be groundings that happen inshore at reduced service speed. The structure is often damaged. And the centre of damage usually occurs in the cargo hold region. Almost one half of the grounding accidents are strandings because the vessel runs on a soft ocean bed.

\subsection{Approaches for stranding accidents}

\section{Generals}

Alsos and Amdahl [1] divided grounding accidents into three different cases depending on the indenter shape (see figure 2.1).
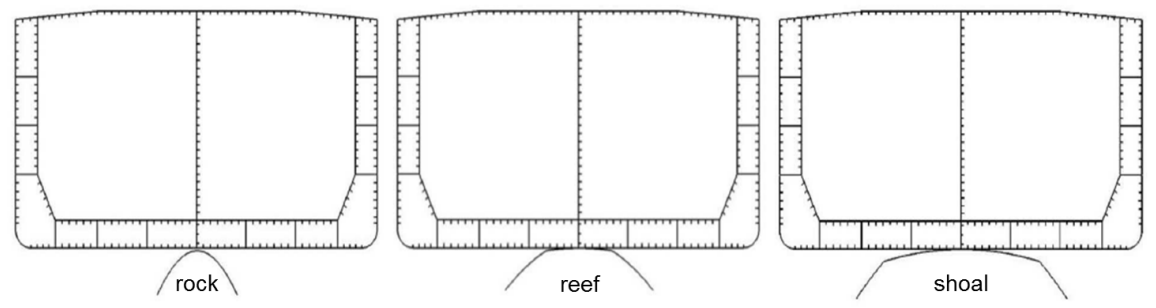

Figure 2.1.: Ocean bed conditions: rock, reef, shoal (Alsos and Amdahl [1]).

Taking the hardness of the ground into the consideration, it decreases while the contact area increases from the left to the right picture. With a bigger contact area the structural damage rises as well. The accident scenario on a shoal is also called stranding or soft grounding. If a ship hits a rock underneath the surface (left picture of figure 2.1), we talk about hard grounding. In this case the structure is immediately damaged. The damage is local e.g. punctuation while the overall structure remains intact. In hard grounding analysis authors have focused on penetration and crushing mechanisms by the hard obstruction (see e.g. Amdahl et al. [2], Kulzep [33], Naar et al. [44] Peschmann [54], Simonsen and Wierzbicki [73], Simonsen [69], [72] and Zhang and Suzuki [85]). This resembles the collision problem.

Simonsen and Wierzbicki [73] and Simonsen [69] presented a mathematical model for calculation of loads and hull girder response during grounding on a rock pinnacle. The computer program "DAMAGE" is based on this part of the work (see also Committee V.1 [10]). Amdahl et al. [2] gave a scenario-based design procedure for hard grounding and collision of tankers. The idea was applied for grounding of two tankers. Two different rigid indenters were forced into the double bottom. The use of the finite element method (FE) was also verified by model tests. Naar et al. [44] compared the crashworthiness of four different bottom structures during hard grounding with the FE programme 
LS-DYNA. Zhang and Suzuki [85] investigated the effect of boundary conditions, shell element type, residual stresses, material model, friction coefficient and rupture strain on the stiffness of a single-hull bottom hit by a rigid wedge shape rock also with the help of LS-DYNA.

\section{Approaches}

The stranding scenario is described by a small number authors. At first works with analytical/empirical approaches are given. Afterwards numerical solutions of the problem are discussed. The chapter closes with a review of grounding experiments.

Lehmann [35] published a formula to estimate the additional bending moment at the mainframe of any ship. The additional bending moment at the mainframe due to different stranding positions is:

$$
\begin{gathered}
M\left(\frac{L_{p p}}{2}\right)=\frac{\Delta T \cdot B \cdot L_{p p}^{2} \cdot c_{W p} \cdot \rho_{w} \cdot g}{8} \cdot\left(4 \frac{e}{L_{p p}}-c_{M}\right) \\
\text { with } \Delta T=T_{0}-\frac{T_{h}+T_{v}}{2}
\end{gathered}
$$

$e$ is the distance between the grounding point and half of the length between perpendicular $L_{p p} / 2$. Furthermore, it is assumed that the maximal bending moment lies at $L_{p p} / 2$, so that the shear forces are zero at $L_{p p} / 2$ in the initial position before grounding. This assumption is correct if the longitudinal centre of gravity $L_{c g}$ lies at $L_{p p} / 2$. The required coefficients $c_{W p}$ and $c_{M}$ are given by Lehmann [35] (pp. 924) for different block coefficients $c_{B}$. They are assumed to be constant for different draught.

Östergaard et al. [47] developed formulas to calculate the additional vertical force $V\left(L^{*}, \tau\right)$ and the additional bending moment $M_{v}\left(L^{*}, \tau, x\right)$ caused by stranding of a pontoon. The formula is based on geometrical examinations.

The additional bending moment is calculated with the following equations:

$$
\begin{aligned}
& \text { for } 0 \leq x \leq\left(L-L^{*}\right) \\
& \qquad \begin{aligned}
M_{v}\left(L^{*}, \tau, x\right) & =\rho g B \cdot\left(\frac{d_{h}-d_{v}}{6 L} x^{3}-\frac{D-d_{v}}{2} x^{2}\right)
\end{aligned} \\
& \text { for }\left(L-L^{*}\right)<x \leq L \\
& \qquad \begin{aligned}
M_{v}\left(L^{*}, \tau, x\right) & =\rho g B \cdot\left(\frac{d_{h}-d_{v}}{6 L}\left(x^{3}-L^{3}\right)-\frac{D-d_{v}}{2}\left(x^{2}-L^{2}\right)\right) \\
& +V\left(L^{*}, \tau\right) \cdot(x-L)
\end{aligned}
\end{aligned}
$$




$$
\text { with } \begin{aligned}
V\left(L^{*}, \tau\right) & =\rho g B L \tau \cdot\left(1+\frac{3}{2} L \cdot\left(0.5-\frac{L^{*}}{L}\right) \cdot f\left(L^{*}, L\right)\right) \\
\text { and } f\left(L^{*}, L\right) & =\frac{\left(L^{*}\right)^{2}-\left(L-L^{*}\right)^{2}}{\left(L^{*}\right)^{3}+\left(L-L^{*}\right)^{3}} \\
d_{h}-d_{v} & =\tau \cdot \frac{3}{2} \cdot L \cdot f\left(L^{*}, L\right) \\
D-d_{v} & =\tau \cdot\left(\frac{3}{2} \cdot\left(L-L^{*}\right) \cdot f\left(L^{*}, L\right)+1\right)
\end{aligned}
$$

The relevant geometrical dimensions are shown in Figure 2.2.

(a)

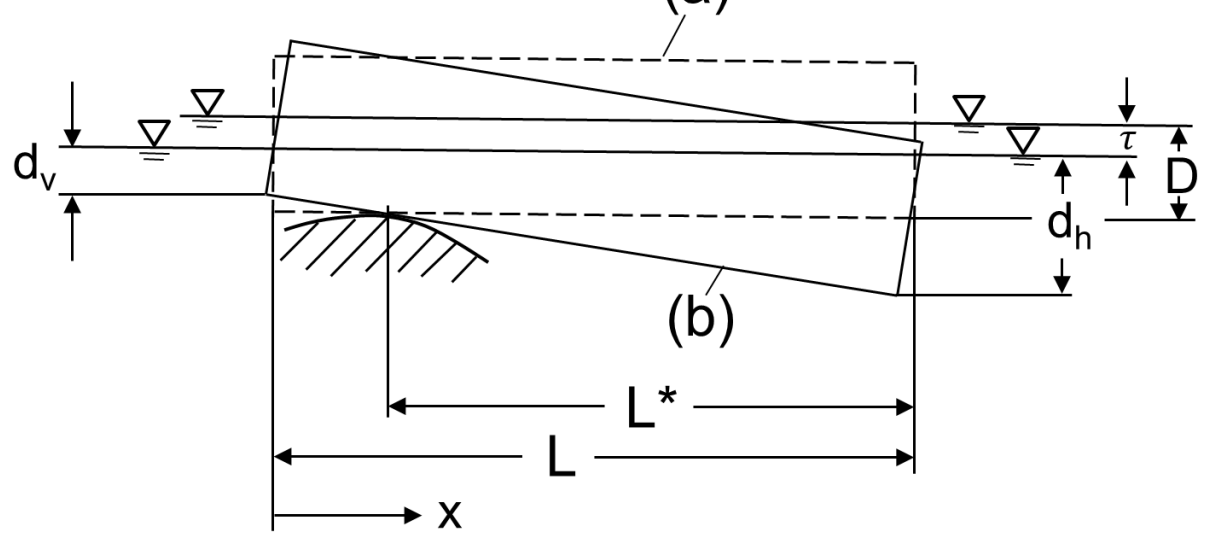

Figure 2.2.: Description of a grounded pontoon (Östergaard et al. [47])

Pedersen [52] presented a mathematical analysis model to calculate the contact force between ship and ground. He divided grounding into two phases. During the first phase the vessel experiences an impulse due to the sudden contact with the sea bottom. Pedersen [52] developed a simplified methodology based on rigid-body motion theory. The assumptions are: an inelastic impulse and a rigid ship. Therefore, the ships forward speed is reduced and it only has surge, heave and pitch velocities. In the second phase the ship slides up the ground slope. The rest of the kinetic energy from the first phase is transformed into friction and potential energy. In the final laying position the contact force is given to be:

$$
\begin{gathered}
F_{z}=\rho g A_{z} \cdot \frac{z}{\left(1+\frac{\ell^{2}}{r^{2}}\right)} \\
\text { with } r=\left(\frac{\nabla \cdot G M_{L}}{A_{z}}\right)^{\frac{1}{2}} \\
\text { and } \ell=\left(x_{c}-s\right)-x_{F}
\end{gathered}
$$

The contact force $F_{z}$ only depends on the vertical displacement of the centre of floatation (LCF). The relevant dimensions are given in figure 2.3, with $z$ for vertical displacement of the hull section, $r$ for equivalent radius of inertia and the typical ship parameter. 


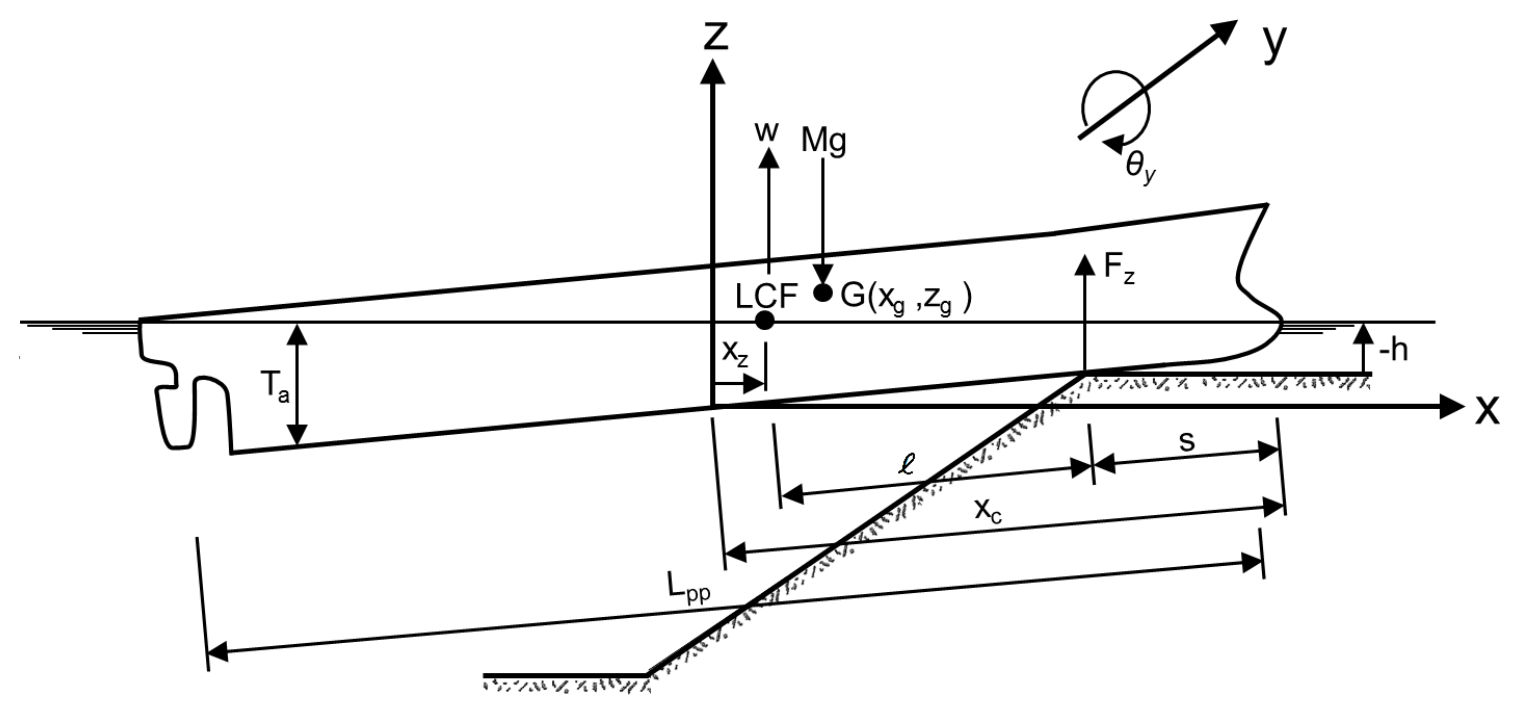

Figure 2.3.: Description of grounded ship (Pedersen [52])

The equation is only valid for small values of $z / T$. Pedersen [52] gave formulas for the additional bending moment (equation 2.4) in the final laying position, too. He assumed that the breadth is constant over the length and that the waterplane area does not change while emerging.

$$
\begin{aligned}
& M(x) \approx \frac{z \rho g A_{z} L}{\left(1+\frac{\ell^{2}}{r^{2}}\right)}\left[( \frac { x } { L } + \frac { 1 } { 2 } ) \left[\frac{l L}{2 r^{2}}\left(\frac{x}{L}\right)\left(\frac{x}{L}-\frac{1}{2}\right)+\frac{1}{2}\left(\frac{x}{L}+\frac{1}{2}\right)\right.\right. \\
& \left.\left.-\frac{4 l}{L}\left\{\left(\frac{x}{L}\right)^{2}-\frac{x}{2 L}+\frac{1}{4}\right\}\right]-\frac{x-x_{c}}{L} H\left(x-x_{c}\right)\right] \\
& \text { with } H\left(x-x_{c}\right)=\left\{\begin{array}{l}
0: x \leq x_{c} \\
1: x>x_{c}
\end{array}\right.
\end{aligned}
$$

For two different tanker vessels Pedersen [52] exemplarily compared the groundinginduced sectional shear forces and bending moments to the wave-induced shear forces and bending moments according to IACS (Nitta et al. [46]) rules. He concluded that grounding incidents of partly loaded ships are often more severe than of fully loaded ships and that the grounding-induced shear forces are more critical than the grounding-induced bending moment. Furthermore, the presented mathematical model was compared to model and full-scale grounding experiments (also see Pedersen and Sterndorff [53]) and good agreement was found. Pedersen [52] also found that large vessels are much more exposed to an overall failure than smaller vessels in a grounding accident.

The publications of Simonsen and Pedersen [70], [71] and Simonsen [69] focused on stranding of ships at the bow. A mathematical model for assessing loads and hull girder response during soft grounding is derived. In Simonsen and Pedersen [70], [69] the reaction of the ground is described. The focus is placed on the interaction between ship and 
ground. They approximated the hydrodynamic pressure forces by constant added-mass terms. The ship hull was assumed to be rigid. Later, Simonsen and Pedersen [71] considered the hydrodynamic pressure forces due to shallow water effects. The method is developed for the dynamics of ships stranding at the bow. The ship is modelled as an elastic beam. So the structural response can be determined as flexural and longitudinal stress waves cause the transient ground reaction and the hydrodynamic forces. Results, which are the furrow of the bow in the sea bed, time variation of the grounding forces, sectional shear forces and bending moments, are presented for a very large crude carrier. The dynamic grounding-induced sectional forces are 15-20\% higher than the forces derived by the method given in Pedersen [52]. Simonsen and Pedersen [70], [69] further presented a mathematical model to calculate the sea bed soil reaction forces on the ship bow. The vessel hits the soft ground with its bow. It is assumed that the penetration of the ship bow generates a flow of pore water through the grain skeleton of the soil. The flow is driven by Dacy's law and by the pressure of the pore water. Besides being subjected to the pore water pressure, the bow is also subjected to the effective stresses in the grain skeleton. The model is implemented in a computer program and compared with experiments. Good agreements of the stopping length, the furrow depths, the vertical soil reaction and the effective coefficient of friction were found.

Relevant works that use numerical approaches to solve parts of the stranding problem are those of Alsos and Amdahl [1] and Reich and Röhr [60], [61], [63].

Alsos and Amdahl [1] computed different grounding and stranding scenarios for a tanker. They modelled three different rigid indenters (see also figure 2.1) which penetrated into the ship bottom at four different locations amidships. By a prior mesh convergence study they got the element size of $150 \mathrm{~mm}$ for the FE simulations. The model had almost two tank lengths and Bernoulli boundary conditions at its ends. The rest of the vessel was not considered. However, in the calculations with the "shoal" indenter they included the global hull bending moment and the flexibility of the ship beam. The bending moment due to grounding and the change of water level is considered via linear considerations. Alsos and Amdahl [1] include the immersion of bow and stern when the ultimate resistance of the girder is reached. Then the girder starts to rotate like a rigid body around the damage location. They assumed that the waterplane area of fore and aft body are identical and do not change with the heave motion. They did not include the influence of the trim. Alsos and Amdahl [1] found that due to the contact with the "shoal" indenter, large parts of the hull structure were deformed, e.g. web crushing and grillage deformation in the double bottom. This leads to a reduced cross section and global bending resulted in buckling of the longitudinal sections. So the interaction of the hogging bending moments and the contact forces affects the longitudinal and penetration resistance of the hull. The skin may not fracture but the overall damage can in the worst case lead to the collapse of the hull beam. Collapse occurred after three to four meters of tidal effects.

Reich and Röhr [60], [61] coupled the FE method with the boundary element method to calculate stranding for a midship section of a tanker. The method was developed to compare two different constructions of the double bottom under extreme loading conditions. The midship section is modelled with finite elements and the ground is represented 
by a boundary integral formation. The vessel comes to the stranding position without any structural damage. In the first step the contact problem is solved. The qualitative distribution of the bedding pressure resulting from the vertical forces (weight, loading and hydrostatic pressure) is calculated. In the second step a global moment and a global shear force are additionally introduced into the model at each end. The global forces are increased until the structure starts to collapse. The characteristics of the ground are given by an isotropic and linear elastic behaviour with three different transversal layers. The computational model is developed further (see Reich and Röhr [63]) to couple external and internal mechanics. This ties in with the idea of Simonsen and Pedersen [70, 71]. In their hybrid computational method a quasi hydro-elastic nonlinear Timoshenko beam model for the hull girder structural responses, and a three-dimensional contact problem model for grounding loads were combined.

The only known large-scale grounding experiment on soft ground was made by Pedersen and Sterndorff [53]. A condemned fishing vessel was used. The experiments were performed on an artificial island and samples were collected from the island's ground. They measured surge, heave and pitch acceleration as well as the deformations of the ground and the ship bow for each test. The vessel suffered no damage during all ten tests. The experiments were used to verify the mathematical models for the external dynamics of stranding presented by Pedersen [52], Simonsen and Pedersen [70], [71] and Simonsen [69].

Two large-scale grounding experiments were conducted by Peschmann et al. [55], Peschmann [54] and Kulzep [33]. A double bottom section of a big tanker was scaled down to 1:3 and fixed at a condemned inland water vessel. The tanker was navigated onto a man-made, hard rock. For the second test the double bottom was filled with foam material. The results, e.g. the energy balance, the deformation and the reaction forces, were compared to non-linear FE calculations. The effect of half filled tanks was also taken into account.

Wang et al. [80] analysed the effect of different rigid indenter shapes and sizes, which represent a rock, through scaled down double bottom grounding experiments. Different penetration locations were used. Four theoretical models were given to calculate the different failure mechanisms. The results are satisfactorily equal to those of the test. The work shows that the outer hull is punctured easily by small indenters, while the internal web configuration is damaged by larger indenters before the shell plating ruptures.

Later Wang [79] published a linear approach to calculate the ultimate hull girder bending moment for vessels in hogging condition and with accidental damage. He simply compared the section modulus $S M^{0}$ of the intact vessel with those of the damaged cross section $S M^{\prime}$. The area of the damaged structure $(A)$ is removed from the cross section area. For grounding the following equation is given:

$$
\frac{S M^{\prime}}{S M^{0}}=1-\alpha_{1}\left(\frac{b}{B}\right) \text { with } \alpha_{1}=\frac{A_{b}}{A}\left(\frac{A z_{0}^{2}}{I}+1\right)
$$

The ship breadth $B$, the breadth of the damaged area $b$, the area of the bottom $A_{b}$ as well as the cross section area $A$, the height of the neutral axis $z_{0}$ and the moment of inertia 
of the intact cross section are needed.

All existing studies simplify the distribution of the hydrostatic forces due to stranding or the ground and can only be applied to a specific grounding position. Furthermore, the presented methods are applied to full-bodied ships. Due to its specific form, it is not possible to calculate the new distribution by hand for a ship without any assumptions. This is why a new method is introduced to calculate the total bending moment, shear force and heeling angle due to grounding for any ship depending on the load case, the grounding point/area and the surface drawdown (see section 3). This method is further used to control the global forces, moments and reactions in the FE simulation of stranding scenarios (see section 4). The soft ground is also modelled. Therefore the three stages of the problem, which are the external dynamics of the grounding event, the internal problem involving structural damage, and the analysis involving the integrity of a stranded ship subjected to tidal actions can be assessed. The first stage can be calculated with the kinetic models of Pedersen [52] and Simonsen [69], [70], [71] or Kulzep [33].

There are no experiments available to verify the presented procedure to simulate stranding accidents within this work. Thus, other tools need to be found or used to verify the results. For example, classification societies evaluate the capacity of the hull girder considering extreme loads to ensure safe design of a ship's hull. The known approaches to achieve the ultimate hull girder strength are given in the next part (see section 2.4).

\subsection{Ultimate hull girder strength}

The maximum capacity, that structural members and systems can sustain is called the ultimate strength. Buckling and yielding dominates the ultimate strength if compressive stress is mainly present. When tensile stress is dominant yielding is the collapse mechanism. We talk about ultimate strength if we consider small structural units. The capacity of a ship's hull on the other hand is measured by its ultimate hull girder strength (UHGS). The longitudinal strength is the ability of a ship structure to resist longitudinal bending under operational and extreme loads without failure. During stranding the structure can be subjected to extreme bending moments and shear forces.

Numerous empirical and analytical methods are available for calculating the ultimate strength of plates, stiffened plates and shells. A good summary is given in Committee III.1 [9]. Most of the design equations are based upon numerical parametric studies and not on test data.

There are two ways to calculate the ultimate hull girder strength under longitudinal bending for a ship. One is to directly calculate the UHGS and the second is to carry out a progressive collapse analysis on a hull girder.

Caldwell [7] as the first to introduce a direct method. He theoretically evaluated the UHGS of a vessel subjected to longitudinal bending. He considered the influence of buckling by reducing the yielding stress of the material at the buckled elements. The ultimate strength is generally overestimated by this method (and later improvements) because it is calculated without considering the strength reduction of the structural members beyond their 
ultimate strength.

In the progressive collapse analysis the strength reduction of each member is taken into account. The best known simplified method for a progressive collapse analysis was presented by Smith [74], nowadays called Smith's method. His idea was to consider the strength reduction of structural members after their ultimate strength and the time lag in collapse of each member. He divided the cross section into small elements (so-called patestiffener combinations). The average stress-average strain relationship of these elements is determined before performing the progressive collapse analysis. The results depend on the accuracy of the average stress-average strain relationship of each element. There are several works that focused on the development of more reliable stress-strain curves (see Committee VI.2 [11]).

Another simplified method is the Idealised Structural Unit Method (ISUM), where the structure is divided into larger structural units. Effective and simple elements are needed to consider the influences of buckling and yielding. These are still under development (see Committee VI.2 [11], Committee III.1 [9]).

It is also possible to simulate a progressive collapse analysis with the FE method. But there are few applications because the influence of material and geometric nonlinearities have to be taken into account and the hull girder may be too large. However, the FE method is the most accurate method for progressive collapse analysis.

\subsubsection{Ultimate hull girder strength check in classification rules}

Most classification societies use Smith's method to predict the UHGS and thus the capacity of the hull. Part of this work was to check whether calculation of classification societies have existing calculation rules that can be expanded to include the influence of shear forces. Besides the Common structural rules (CSR), the rules of the following classification societies are considered: Bureau Veritas (BV), Det Norske Veritas (DNV), Germanischer Lloyd (GL), Lloyd's Register (LR) and Nippon Kaiji Kyokai (ClassNK).

A comparison of the various rules is performed. With the exception of $D N V$, all societies mentioned adopted the procedure described in the Common structural rules for bulk carriers (CSR-Bulkers) [26] with small modifications. Therefore, the CSR-Bulkers form the basis of the comparison and are described in detail.

\section{UHGS check in the Common structural rules for bulk carriers}

The sum of still water and vertical wave bending moment has to be smaller then the vertical ultimate bending moment $M_{U}$ divided by a partial safety factor $\gamma_{R}$. The ultimate bending moment capacities of a hull girder transverse section are defined as the maximum values of the moment-curvature curve. The bending moment capacity $M_{U}$ versus the curvature $\chi$ of the transverse section is calculated via Smith's method. The algebraic sign of the curvature depends on the load case, positive for hogging and negative for sagging. The applied curvature, which equals the rotation angle of the hull girder transverse section around its horizontal neutral axis, is incrementally increased and induces axial strain. The basic procedure is divided into seven steps. 
First the longitudinal units of the structure are divided into stiffened elements. Three kinds of elements are defined: ordinary stiffeners (stiffener and connected plate), hard corners (in general two plates that are in different planes) and plating panels (lies in between ordinary stiffeners, hard corners or between ordinary stiffeners and hard corners). For each element the stress-strain relationships is defined depending on the mode of failure in the second step. Third, the curvature and the neutral axis for the first increment step need to be found with the value of the incremental curvature that induces a stress equal to $1 \%$ of the yield strength in the strength deck. In the fourth step the strain $\epsilon_{i}$ and stress $\sigma_{i}$ depending on the curvature $\chi_{i}$ is calculated for each element. Fifth, the neutral axis is determined at each incremental step by establishing force equilibrium over the whole transverse section. In the sixth step the contributions given by the stress $\sigma_{i}$ acting on each element is summed to obtain the moment $M_{i}$. Lastly the moment in the current incremental step is compared with the moment in the previous incremental step. If the slope in the moment-curvature curve is less than a negative fixed value the peak value is the searched ultimate bending moment. Otherwise the curvature is increased and the analysis is done again beginning at the fourth step.

Several assumptions are made. Among others, the ultimate strength is calculated at hull transverse sections, that remain plane during each increment, between two transverse webs. The material exhibits an elasto-plastic behaviour. The stress-strain curves are calculated for the failure mechanisms of the element and the lowest stress is selected of the values that are obtained from each of the considered load-end shortening curves. The failure mechanisms are: ideal elasto-plastic collapse, beam column buckling, torsional buckling, web local buckling of flanged profiles, web local buckling of flat bars and plate buckling. The effects of shear force, torsion force, horizontal bending and side pressure are neglected. Initial imperfections i.e. pre-deformation and residual stress are not considered.

The UHGS is calculated for sagging and hogging in intact, harbour and flooded conditions.

\section{Difference in the UHGS check of various classification societies}

The differences are described with respect to the UHGS check of the CSR-Bulkers. The most significant differences are also summarised in table 2.2 and 2.3.

- The incremental UHGS check of the Common structural rules for tanker (CSRTankers) [25] is similar to the method for CSR-Bulkers. It is only required to check the UHGS for sagging in the intact condition. The factors in the empirical determination of the vertical bending moment and the safety factors are a little different. The incremental curvature step is different and the CSR-Tankers do not differentiate between the yield stress in plates or stiffeners.

In addition to the progressive collapse analysis a simplified single step procedure that is based on a reduced hull girder bending stiffness accounting for buckling of the deck can be applied to calculate the sagging UHGS. If the assumption that the ultimate sagging capacity is the point at which the ultimate capacity of the stiffened deck panels is reached is fulfilled by the structural configuration this procedure is valid. 
- Bureau Veritas $(B V)[6]$ gives slightly different safety factors. There is no difference defined for the yield stress of plates or stiffeners. For the failure mechanism 'torsional buckling' the Euler torsional buckling stress is given in another way. The UHGS check needs to be performed for sagging and hogging cases in intact and harbour conditions.

- In the rules of Germanischer Lloyd (GL) [18] the incremental UHGS check has more differences to the CSR-Bulkers. The method is partly based on DIN 18800 norm (see Committee III.1 [8]). The effective width $b_{m}$ has to be determined according to the buckling strength analysis. The failure mechanism 'buckling of flat bars' is not considered. The slenderness ratio is additionally dependent on a buckling factor. The critical stresses for beam column and torsional buckling are differently defined. The failure mechanism 'plate buckling' is calculated with other definitions. $G L$ has also own safety factors and an other curvature definition. The yield stress for plates and stiffeners is equal. The check is not necessary for harbour conditions.

- The classification society Lloyd's Register (LR) [39] has no rules of its own. If the UHGS check is necessary (for bulkers and tankers), the procedures of the CSR are accepted.

- Nippon Kaiji Kyokai (ClassNK) [45] adopted the CSR-Bulkers and Tankers. The safety factors are defined differently.

Besides those rules ClassNK has a single step method based on simplified equations. It can be applied if the deck and bottom longitudinal stiffeners are arranged at almost the same interval, and that deck/bottom plating thickness is almost the same. For bulker and container vessels the yield stress is not the same in stiffeners and plates.

- For vessels over $100 \mathrm{~m}$ in length, that are classified by Det Norske Vertias (DNV) [12], a different UHGS check has to be performed. The method is not based on Smith's method. For the buckling modes of plates and stiffened plates $D N V$ uses the elastic large deflection plate theory of Marguerre and von Karman in its program PULS (for more details see Committee III.1 [8]). The bending moment capacity $M_{U}$ is the sum of an elastic moment capacity $M_{E}$ and an additional moment due to the increase in allowable stress above the elastic buckling limit $\Delta M_{U}$. There is an additional moment because plate elements subjected to extreme loading can have in-plane compressive stresses above the elastic buckling stress. Thereby, functional requirements are not allowed to prohibit large and off-plane elastic deflections.

At first the maximum allowable compressive stress is calculated for each local panel where elastic buckling is expected. This depends on the elastic buckling stress and an excess factor. For the elastic buckling stress the buckling factor is determined in the same way as described in CSR-Bulkers. Afterwards the effective breadth is derived, because elastic buckling reduces the effective breadth of plates taking part in the compression area. Now the bending moment capacity can be determined with respect to the plastic neutral axis.

The check is only necessary for intact conditions. The yield stresses of plates and stiffeners is different. 


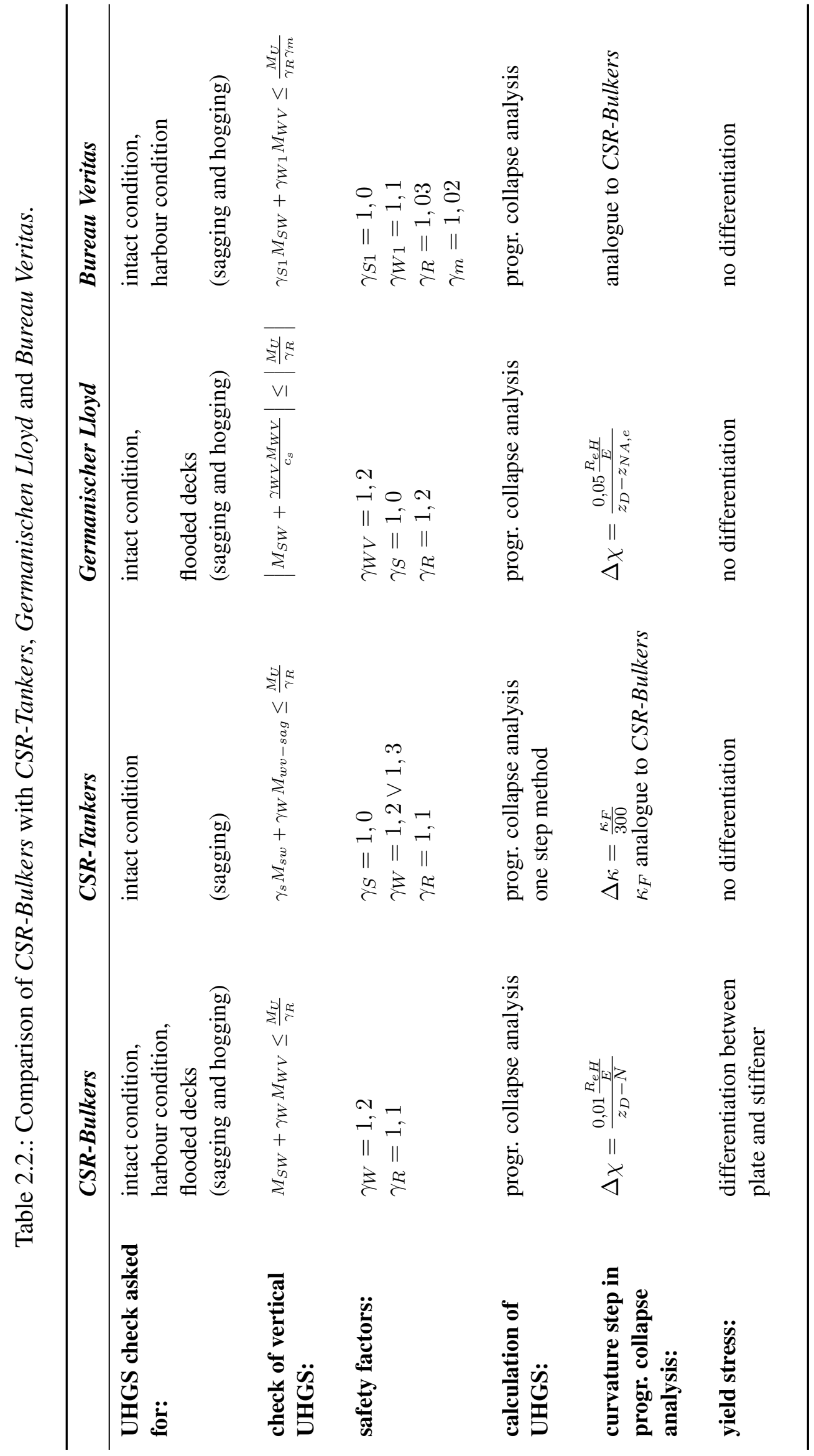




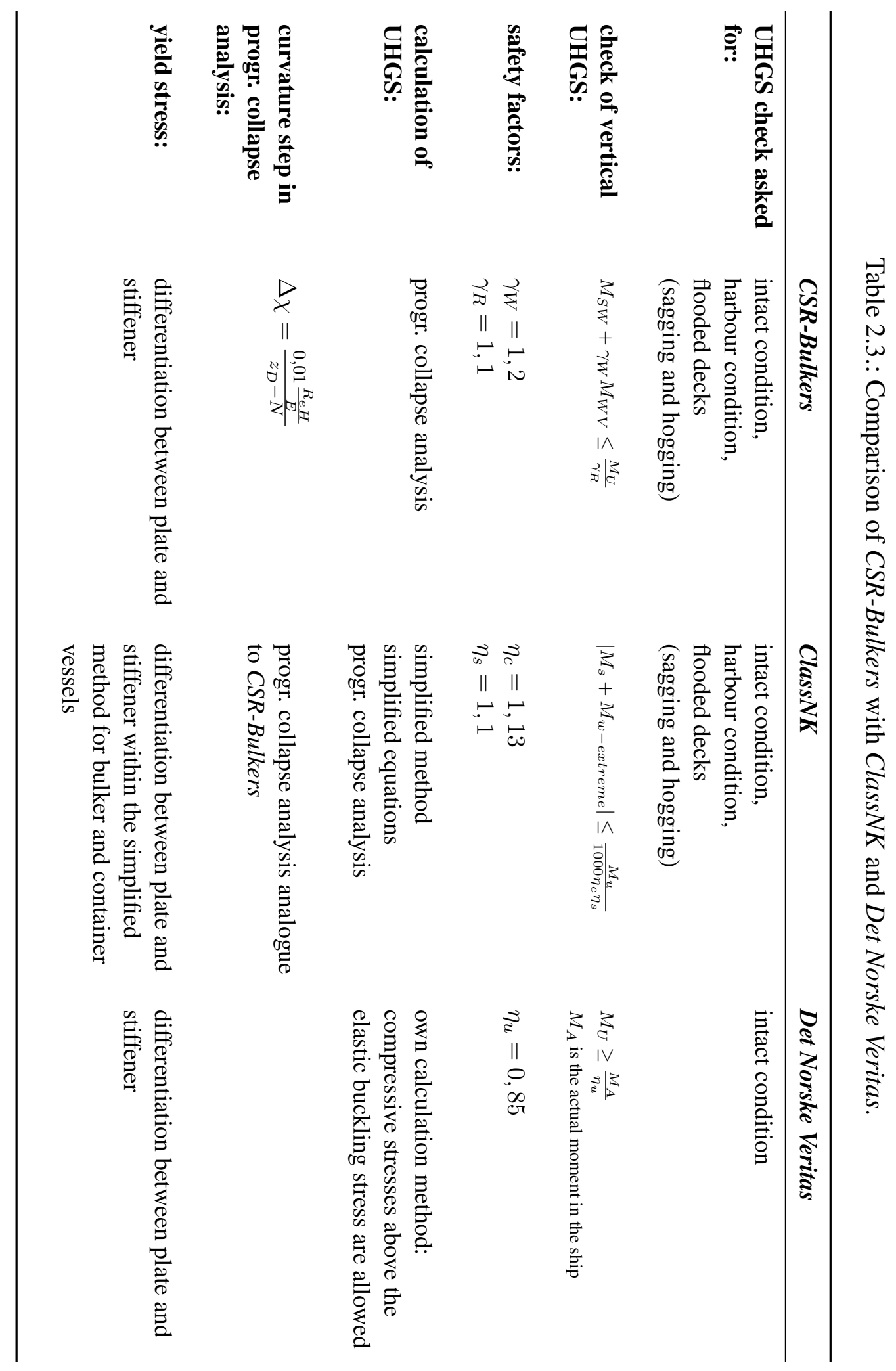


The ultimate hull girder strength is calculated for a cross section of a container vessel with the programs POSEIDON (GL), MARS2000 (BV) and NAUTICUS (DNV). The relevant dimensions of the 4300 TEU POSTPANMAX container vessel (called Postpanmax) built in 2009 are given in table 2.4 .

It is possible to additionally calculate the UHGS based on CSR-Bulkers and CSR-Tankers with the program Nauticus. This allows a comparison of the qualitative differences of the UHGS checks based on CSR-Bulkers, CSR-Tankers, GL, BV and DNV. Although a container vessel is typically in hogging the UHGS is also calculated for the sagging condition.

Table 2.4.: Dimensions of Postpanmax.

\begin{tabular}{lr}
\hline length $L_{o a}[\mathrm{~m}]$ & 294.10 \\
length $L_{p p}[\mathrm{~m}]$ & 285.60 \\
breadth $B[\mathrm{~m}]$ & 40.00 \\
height $D[\mathrm{~m}]$ & 24.20 \\
design draught $T_{d}[\mathrm{~m}]$ & 12.04 \\
block coefficient $c_{B}$ & 0.656 \\
mainframe coefficient $c_{M}$ & 0.98 \\
speed $v[\mathrm{kN}]$ & 24.00 \\
\hline
\end{tabular}

At first the geometrical results are presented in table 2.5. The moment of inertia $I_{y}$, the height of the elastic neutral axis from the base line $z$, the section modulus of the bottom $W_{B}$ at $z=0$ and the section modulus of the deck $W_{D}$ at $z=24.2 \mathrm{~m}$ are different. The programs are based on different calculation methods and have rounding errors. Therefore, it is not remarkable that the still water $\left(M_{S W}\right)$ and wave induced vertical bending moments $\left(M_{W V}\right)$ for sagging and hogging are different (see table 2.6). $M_{\text {hog }}$ and $M_{s a g}$ are the resultant bending moments at $z=0 . \gamma_{W}$ is a safety factor.

The ultimate capacity of the hull under hogging $\left(M_{U, h o g}\right)$ and sagging $\left(M_{U, \text { sag }}\right)$ is presented in table 2.8. The factors $f_{U S, s a g}$ and $f_{U S, h o g}$ reveal the ratio of the actual moment from the requested moment. For example, for hogging the equation is:

$$
f_{U S, h o g}=\frac{M_{h o g} \cdot \gamma_{R}}{M_{U, h o g}}>1 \rightarrow \text { not acceptable }
$$

The smaller the $f_{U S, s a g}$ or $f_{U S, h o g}$ the larger the reserve of the cross section against collapse. Values above one are not acceptable. In the rules of $D N V$ the security factor is $\eta_{u}$ (see table 2.3) whereas $\gamma_{R}$ is equal to $1 / \eta_{u}$.

In the hogging condition the vessel has the largest UHGS if it is calculated according to $C S R$-Bulkers, CSR-Tankers or $B V$ that assume a similar value for $f_{U S, h o g}$. The smallest UHGS is predicted via the rules of $D N V$. The results of $G L$ lie in between.

For sagging the predicted UHGS is smaller than for hogging. Only from the viewpoint of $D N V$ is the UHGS higher under hogging. The lowest factor $f_{U S, h o g}$ is achieved with the rules of $B V$ and the highest with the rules of $G L$. According to $G L$ the structure is not secure in extreme sagging conditions. All other rules lie in between those of $B V$ and $G L$. The difference arises because $G L$ refers the section modulus of the deck to a higher 
z-position.

Table 2.5.: Geometry and section modulus.

\begin{tabular}{lrrrr}
\hline & $\mathbf{I}_{\mathbf{y}}\left[\mathrm{m}^{4}\right]$ & $\mathrm{z}[\mathrm{m}]$ & $\mathbf{W}_{\mathbf{B}}\left[\mathrm{m}^{3}\right]$ & $\mathbf{W}_{\mathbf{D}}\left[\mathrm{m}^{3}\right]$ \\
\hline CSR-B & 456.78 & 10.43 & 43.79 & 33.17 \\
CSR-T & 458.55 & 10.41 & 44.07 & 33.24 \\
GL & 478.33 & 10.39 & 46.05 & 31.17 \\
BV & 477.31 & 10.28 & 46.44 & 34.28 \\
DNV & 475.69 & 10.30 & 46.19 & 34.22 \\
\hline
\end{tabular}

Table 2.6.: Bending moments for hogging.

\begin{tabular}{lrrrr}
\hline & $\mathbf{M}_{\mathbf{S W}}[\mathrm{kNm}]$ & $\mathbf{M}_{\mathbf{W V}}[\mathrm{kNm}]$ & $\boldsymbol{\gamma}_{\mathbf{W}}$ & $\mathbf{M}_{\text {hog }}[\mathrm{kNm}]$ \\
\hline CSR-B & 3390951 & 4007886 & 1.20 & 8200414 \\
CSR-T & 3221834 & 4007886 & 1.20 & 8031297 \\
GL & 3390851 & 4009026 & 1.20 & 8201682 \\
BV & 3390951 & 4007887 & 1.10 & 7799627 \\
DNV & 3390951 & 4007886 & 1.00 & 7398139 \\
\hline
\end{tabular}

Table 2.7.: Bending moments for sagging.

\begin{tabular}{lrrrr}
\hline & $\mathbf{M}_{\mathbf{S W}}[\mathrm{kNm}]$ & $\mathbf{M}_{\mathbf{W V}}[\mathrm{kNm}]$ & $\gamma_{\mathbf{W}}$ & $\mathbf{M}_{\text {sag }}[\mathrm{kNm}]$ \\
\hline CSR-B & 2748139 & 4650697 & 1.20 & 8328975 \\
CSR-T & 2192170 & 4650697 & 1.20 & 7773006 \\
GL & 2741894 & 4657983 & 1.20 & 8331473 \\
BV & 2748140 & 4650698 & 1.10 & 7863908 \\
DNV & 2748139 & 4650697 & 1.00 & 7398836 \\
\hline
\end{tabular}

Table 2.8.: Comparision of UHGS of the checked classification rules.

\begin{tabular}{lccrrr}
\hline & $\mathbf{M}_{\mathbf{U}, \text { hog }}[\mathrm{kNm}]$ & $\mathbf{M}_{\mathbf{U}, \mathbf{s a g}}[\mathrm{kNm}]$ & $\gamma_{\mathbf{R}}$ & $\mathbf{f}_{\mathbf{U S}, \text { hog }}$ & $\mathbf{f}_{\mathbf{U S}, \text { sag }}$ \\
\hline CSR-B & 12954458 & 10248029 & 1.10 & $0.70(3)$. & $0.89(4)$. \\
CSR-T & 13029483 & 10277215 & 1.10 & $0.68(1)$. & $0.83(2)$. \\
GL & 11480584 & 8804525 & 1.20 & $0.86(4)$. & $1.14(5)$. \\
BV & 11855100 & 11154100 & 1.05 & $0.69(2)$. & $0.74(1)$. \\
DNV & 9601559 & 10085414 & 1.18 & $0.91(5)$. & $0.86(3)$. \\
\hline
\end{tabular}


All discussed methods do not consider the effect of shear force, torsion force, horizontal bending and side pressure. To estimate the influence of stranding for the UHGS the shear force should be taken into account, at least.

The class rules are based on the incremental increase of the curvature, no load distribution is considered. Therefore it is not possible to derive the acting shear force. It is however possible to introduce a fictive shear force that is incrementally increased together with the fictive bending moment $M_{i}$. In each incremental step the effective cross section-area of the stiffened elements could be reduced due to loading with shear forces and then the rest of the cross section is taken for calculating the ultimate bending moment.

Although it is possible to expand the existing rule programs to include the shear force in the calculation of the UHGS, this option is not chosen. The moment-shear force interaction would depend on the assumption of the fictive shear force. The change of the global bending moment due to stranding would not be considered. The programs are optimised for the midship section and are not open source.

\subsubsection{Interaction of ultimate bending moment and shear force}

Especially the additional shear force at the stranding area has an influence on the ultimate hull girder strength. For symmetric beam structures, e.g. with double T-sections or full cross sections, interaction curves can be found mainly in the field of civil engineering (see among others Drucker [14], Petersen [56], Reckling [58], Schneider et al. [67], Windels [82], Windels [83]). Usually the effective cross section is reduced due to loading with shear forces and then the rest of the cross section is taken for calculating the ultimate bending moment. The method is easily applicable because the elastic neutral axis is equal to the plastic neutral axis.

Paik et al. [51] published analytical formulas to calculate the ultimate capacity under pure bending moment or shear forces for ship-like cross sections. A moment-shear force interaction curve is empirically derived for combined loading. Different cross sections are modelled and calculated with ISUM. The shear force is first applied incrementally and fixed at a certain value and then the moment is applied incrementally. The curves also depend on different ship types as well as on a partly different relation of applied bending moment and shear force. The formula for the ultimate bending moment is based on an earlier publication (Paik [48]) and was expanded for every cross section type. It is also compared to other analytical formulas, such as that of Caldwell [7], which overestimates the ultimate bending moment in general. Caldwell [7] suggested the formula for a simple cross section without double bottom or additional decks.

\section{Ultimate moment and shear force capacity after Paik et al. [51]}

For a pure hogging moment the ultimate moment capacity is given by Paik et al. [51] in the following equation (2.7) whereas the coordinate system is changed to the standard known coordinate system in shipbuilding ( $x$ : ship length, $y$ : ship breadth, $z$ : ship height): 


$$
\begin{aligned}
M_{V u h} & =A_{z 0} \sigma_{u B}(D-g)+A_{z 1} \sigma_{u I B}\left(D-z_{1}-g\right) \\
& -\frac{1}{H}\left(\sigma_{u S L}+\sigma_{z S U}\right)\left[\sum_{i=2}^{m-1} A_{z i}\left(D-z_{i}\right)\left\{g-\left(D-z_{i}\right)\right\}\right] \\
& -\sigma_{z S U}\left[\sum_{i=2}^{m-1} A_{z i}\left(D-z_{i}-g\right)\right]+\frac{\sigma_{u S L}}{2 D}\left(\sum_{j=0}^{n} A_{y j}\right)(D-H)(D+H-2 g) \\
& +A_{z m} \sigma_{z D} \cdot g+\frac{H}{6 D}\left(\sum_{j=0}^{n} A_{y j}\right)\left[(2 H-3 g) \sigma_{u S L}-(H-3 g) \sigma_{z S U}\right]
\end{aligned}
$$

with $g=\frac{\sigma_{z S U} \cdot H}{\sigma_{u S L}+\sigma_{z S U}}$

$$
\begin{aligned}
H & =C_{1} D+\sqrt{C_{1}^{2} D^{2}+2 C_{2} D} \\
C_{1} & =\frac{A_{z 0} \sigma_{u B}+A_{z 1} \sigma_{u I B}+\sigma_{u S L}\left(\sum_{j=0}^{n} A_{y j}\right)-A_{z m} \sigma_{z D}-\sigma_{z S U}\left(\sum_{i=2}^{m-1} A_{z i}\right)}{\left(\sigma_{u S L}+\sigma_{z S U}\right)\left(\sum_{j=0}^{n} A_{y j}\right)} \\
C_{2}= & \frac{\sum_{i=2}^{m-1} A_{z j}\left(D-z_{i}\right)}{\sum_{j=0}^{n} A_{y j}}
\end{aligned}
$$

All relevant variables are plotted in figure 2.4 and are:

$A_{y i}$ : sectional area of vertical members at $y=y_{i}$

$A_{z i}$ : sectional area of horizontal members at $z=z_{i}$

$\sigma_{u D}, \sigma_{u B}, \sigma_{u I B}, \sigma_{u S U}, \sigma_{u S L}:$ ultimate compressive stress of panels at deck, outer bottom, inner bottom, upper and lower side shell

$\sigma_{z D}, \sigma_{z B}, \sigma_{z S U}, \sigma_{z S L}:$ yield stress of outer bottom, inner bottom, upper and lower side shell

$D$ : depth of ship

$g$ : neutral linear elastic axis above base line 


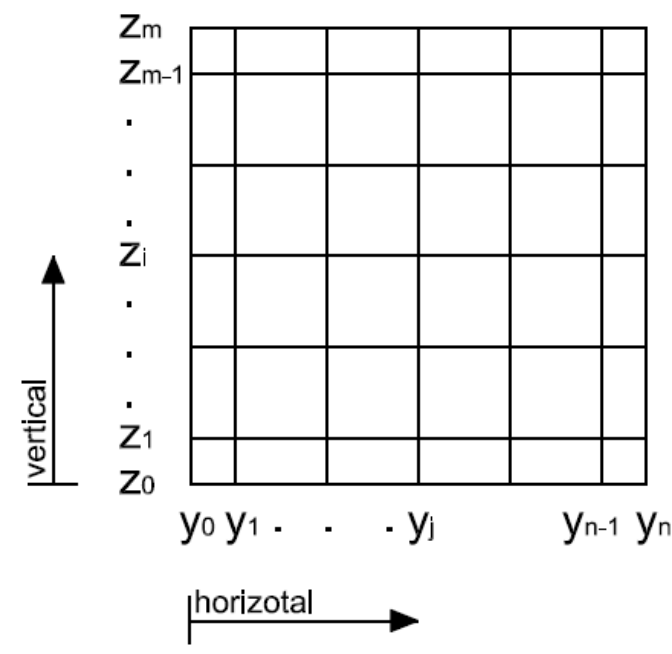

(a) cross section

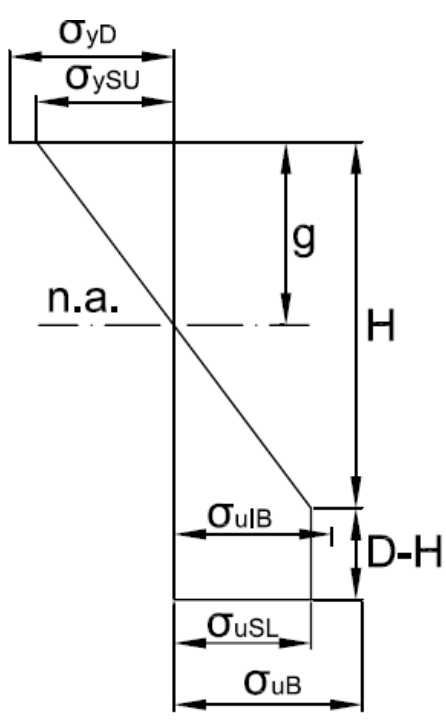

(b) hogging

Figure 2.4.: Assumed longitudinal stress over hull cross section (Paik et al. [51]).

The ultimate compressive stresses $\sigma_{u}$ can be calculated via an empirical formula, given in Paik [48]:

$$
\sigma_{u}=\sigma_{z}\left(0.995+0.936 \lambda^{2}+0.17 \beta^{2}+0.188 \lambda^{2} \beta^{2}-0.067 \lambda^{4}\right)^{-0.5}
$$

The plate slenderness ratio $\beta$, the column stiffened slenderness ratio $\lambda$ and the yield stress $\sigma_{y}$ are defined as:

$$
\begin{aligned}
\beta & =\frac{b^{\prime}}{t} \sqrt{\frac{\sigma_{z}}{E}} \\
\lambda & =\frac{a}{\pi r} \sqrt{\frac{\sigma_{z}}{E}} \\
\sigma_{y} & =\frac{b^{\prime} t \sigma_{z p}+A_{s t} \sigma_{z s}}{b^{\prime} t+A_{s t}}
\end{aligned}
$$

$b^{\prime}$ : spacing between longitudinal stiffeners

$t$ : plate thickness

$E$ : Young's modulus

$a:$ length of stiffened panel between transverse frames

$r:$ radius of gyration of stiffener with full plating

$\sigma_{z p}, \sigma_{z s}:$ yield stress of plate and stiffener

$A_{s t}$ : cross section area of one stiffener alone 
The ultimate shear force $Q_{V u}$ is given by the known approach in equation (2.10) and the ultimate shear stress $\tau_{u}$ of a rectangular plate between stiffeners was published by Paik and Lee [49] (see equation (2.11)).

$$
\begin{gathered}
Q_{V u}=2 A_{s} \tau_{u} \\
\tau_{u}=\tau_{c r} \cdot \alpha \cdot \frac{d_{2}-\sqrt{d_{2}^{2}-4 d_{1} d_{3}}}{2 d_{1}}
\end{gathered}
$$

with $d_{1}=0.0126 \cdot \tau_{z}$

$d_{2}=0.1067 \cdot \tau_{z}+\tau_{c r}$

$d_{1}=1.084 \cdot \tau_{z}$

$\alpha$ accounts for the influence of distortion of stiffeners and is set to 0.9. The critical shear buckling stress $\tau_{c r}$ in equation (2.11) is achieved by a plasticity correction to the elastic shear buckling stress $\sigma_{z}$ given by American Bureau of Shipping [3] and the elastic shear buckling stress $\tau_{E}$ is calculated according to Bleich [5] in equation (2.13).

$$
\begin{array}{crl}
\tau_{c r}=\tau_{E} & \text { for } \frac{\tau_{E}}{\tau_{z}} \leq 0.5 \\
\tau_{c r}=\tau_{z}\left(1-\frac{\tau_{z}}{4 \tau_{E}}\right) & \text { for } \frac{\tau_{E}}{\tau_{z}}>0.5 \\
\tau_{E}=k_{\tau} \cdot \sigma_{E} & \\
\text { with } \sigma_{E}=\frac{\pi^{2} E}{12\left(1-\nu^{2}\right)}\left(\frac{t}{b^{\prime}}\right)^{2} & \\
\text { and } k_{\tau}=5.34+4\left(\frac{b^{\prime}}{a}\right)^{2} & \text { for } \frac{a}{b^{\prime}} \geq 1 \\
k_{\tau}=5.34+4\left(\frac{a}{b^{\prime}}\right)^{2} & \text { for } \frac{a}{b^{\prime}}<1
\end{array}
$$

A moment-shear force interaction curve is empirically derived on the basis of the calculated values $M_{V u}$ under pure bending moment and $Q_{V u}$ under pure shear force loading whereas the factors are given as $c_{3}=2$ and $c_{4}=5$ :

$$
\left(\frac{M_{V}}{M_{V u}}\right)^{c_{3}}+\left(\frac{Q}{Q_{V u}}\right)^{c_{4}}=1
$$

Yao et al. [84] expanded their computer program HULLST, which is based on Smith's method, to calculate the influence of the shear stress and warping due to alternate loading. 
In both publications (Paik et al. [51] and Yao et al. [84]) the interaction of bending moment and shear force is examined under normal loading conditions.

There are few simple methods/formulas to estimate the influence of the shear force in combination with the acting bending moment for ship hull-like structures. Here the elastic and plastic neutral axis are different. The above mentioned methods are based on non-commercial computer programs which are not available to the author. Furthermore, the proposed methods are not for extreme loading conditions. Thus, an own method is developed based on an experiment (see section 5) to estimate the influence of the shear force on the ultimate bending moment for diverse cross sections. The aim is to predict the reserve of the ultimate capacity when the acting bending moment due to combined loading is known.

Schultz [68] tested transversely stiffened box girder models with a deck opening under pure bending. He determined the buckling and post buckling behaviour. The specimens had huge pre-deformations due to the fabrication. One longitudinal stiffened box girder was tested by Gordo and Soares [19] also when loaded with a bending moment. The specimens had their stiffeners on the outside of the box. A focus was placed on the residual stresses. Reckling et al. [59] tested seven different box girders with longitudinal stiffeners under pure bending load to determine the ultimate strength. The results were compared to existing formulas. This experiment is chosen for recalculation because all relevant data are given.

\section{Pure bending moment experiment by Reckling et al. [59]}

\section{Experimental setup:}

Seven specimens were used with a length between 1210 and $1800 \mathrm{~mm}$ and different cross sections. Three of them are recalculated with FEM (see section 5). The relevant geometrical data are given in table 2.9 and figure 2.5.

Table 2.9.: Dimensions of specimen cross section used by Reckling et al. [59].

\begin{tabular}{|c|c|c|c|c|c|c|}
\hline \multirow[t]{2}{*}{ no } & \multirow{2}{*}{$\begin{array}{c}\ell_{\mathbf{k}} \\
{[\mathrm{mm}]}\end{array}$} & \multirow{2}{*}{$\begin{array}{c}\mathbf{t} \\
{[\mathrm{mm}]}\end{array}$} & \multicolumn{2}{|c|}{ stiffeners } & \multirow{2}{*}{$\begin{array}{c}\mathbf{b}^{\prime} \\
{[\mathrm{mm}]}\end{array}$} & \multirow{2}{*}{$\begin{array}{c}\mathbf{h} \\
{[\mathrm{mm}]}\end{array}$} \\
\hline & & & top/bottom [mm] & side shell [mm] & & \\
\hline 31 & 1410 & 2.5 & $\begin{array}{l}4 \text { L profiles } \\
30 \times 20 \times 3\end{array}$ & $\begin{array}{l}2 \text { I profiles } \\
30 \times 2.5\end{array}$ & 120 & 133 \\
\hline 22 & 1210 & 2.5 & $\begin{array}{l}6 \mathrm{~L} \text { profiles } \\
30 \times 20 \times 3\end{array}$ & $\begin{array}{l}3 \text { I profiles } \\
30 \times 2.5\end{array}$ & 85.7 & 100 \\
\hline 23 & 1410 & 2.5 & $\begin{array}{l}6 \mathrm{~L} \text { profiles } \\
30 \times 20 \times 3\end{array}$ & $\begin{array}{l}3 \text { I profiles } \\
30 \times 2.5\end{array}$ & 85.7 & 100 \\
\hline
\end{tabular}

Each specimen has two transverse stiffeners which are located at $\mathrm{x}= \pm 250 \mathrm{~mm}$ (see figure 2.6). The used coordinate system is given in figures 2.5 and 2.6. All specimens collapse at the top plate between the two transverse stiffeners. 


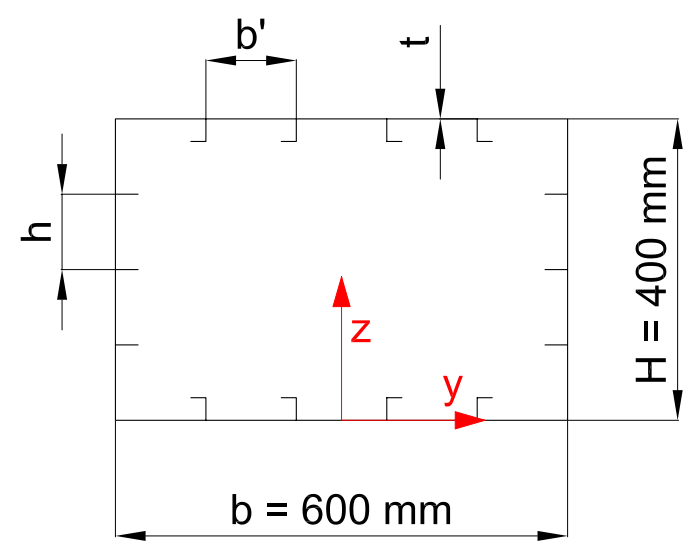

Figure 2.5.: Cross section of specimen used by Reckling et al. [59].

Most specimens are connected via box-shaped subcarriers with the HEB-400 beams. The HEB beams (European wide flange beams) are produced in accordance with DIN-1025. The subcarriers have the same cross section as its specific specimen. Three specimens, among number 31, are directly connected with the HEB beam. For the connection transverse plates are welded on each end of the specimens, of the subcarriers and of the HEB beams. The plates are screwed together.

The length between the bearings as well as the distance between the bearing and the load introduction are always the same. The load is applied via two hydraulic pillars, which have an increasing velocity of $0.3 \mathrm{~mm} / \mathrm{min}$ during the experiment. The experimental setup is shown in figure 2.6.

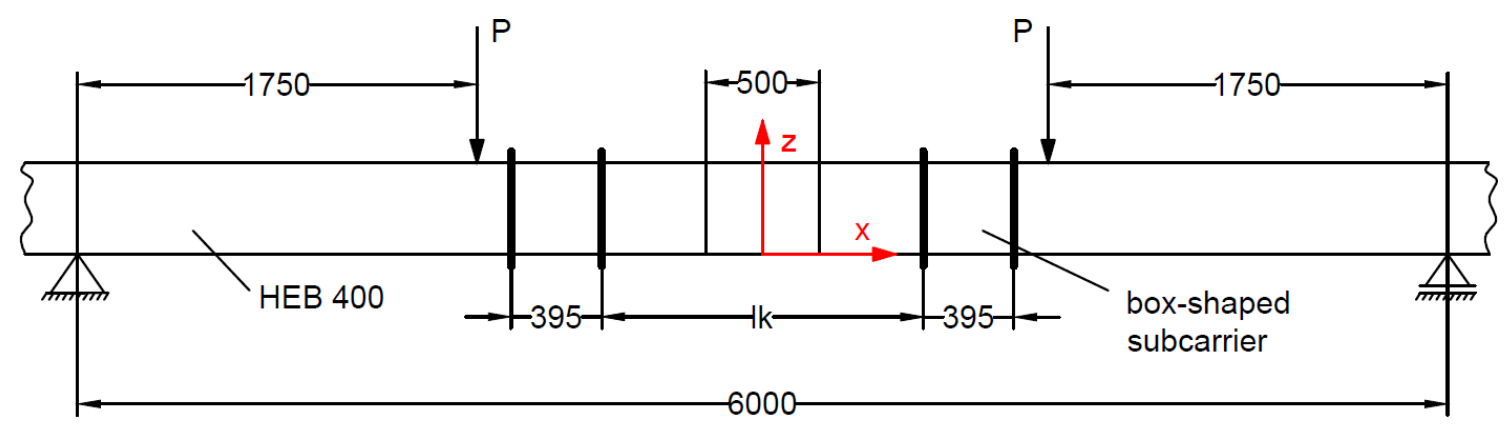

Figure 2.6.: Experimental setup used by Reckling et al. [59].

Except for one pilot test specimen all specimens are subjected to stress relief heat treatment to reduce the pre-deformation. The top plate's residual pre-deformation of specimen 31, 22 and 23 were gained by the Moiré-procedure [24] after the treatment.

To determine the yielding point of each specimen, tension tests with sheet metal samples after DIN standard were carried out. The sheet metal samples were also treated with stress relief annealing. The relevant results, which are needed for the FE calculation, are given in table 2.10 . 
Table 2.10.: Results of tension test and reference value given by Reckling et al. [59].

\begin{tabular}{ccccc}
\hline no & $\sigma_{\mathrm{F}}\left[\mathrm{N} / \mathrm{mm}^{2}\right]$ & $\mathrm{E}\left[\mathrm{N} / \mathrm{mm}^{2}\right]$ & $\mathbf{M}_{\mathbf{e}}[\mathrm{kNm}]$ & $\mathbf{f}_{\mathbf{e}}[\mathrm{mm}]$ \\
\hline $\mathbf{3 1}$ & 255 & $2.09 \cdot 10^{5}$ & 231.7 & 15 \\
$\mathbf{2 2}$ & 255 & $2.09 \cdot 10^{5}$ & 256.6 & 11.1 \\
$\mathbf{2 3}$ & 239 & $2.09 \cdot 10^{5}$ & 240.5 & 14.1 \\
\hline
\end{tabular}

\section{Analysis of tests:}

Normalised moment-displacement curves and the deformation of the top plate are given. The measured moments and displacements are divided by the elastic reference value. The elastic limit moment $M_{e}$ and the appendant displacement $f_{e}$ are calculated for a hypothetical girder, which has the same cross section as the corresponding test specimen and can be loaded by the plastic limit moment $M_{p}$ without buckling until full plasticity is reached. Both calculated reference values are given in table 2.10.

Recalculations by ANSYS [4] and MARC MENTAT [43] revealed that the displacement $f_{e}$ is given with a wrong unit in the paper. In table 2.10 the units are corrected.

The normalised moment-displacement curves of all tests have a smaller gradient than the curves of the equivalent hypothetical girders. The authors state that the different gradients are due to pre-deformations and manufacturing-related initial stresses, that are just partly reduced by the stress relief heat treatment.

All specimens collapse due to buckling in the top plate between the transverse structures. The top plate's deformation of specimen 31, 22 and 23 are given after the ultimate strength is reached and again gained by the Moiré-procedure.

The test series revealed that one cannot directly conclude from the pre-deformation to the appearance of buckles after reaching the ultimate bending moment.

\subsection{Finite element method}

As already documented in the previous sections, many complex mechanical problems are nowadays solved by the finite element method (FEM). Static and dynamic problems can be modelled with various degrees of accuracy depending on the calculating capacity. To use the method correctly knowledge in the field of mechanics is needed. The basic idea of the method is to divide the structure into finite elements. There are diverse element types, such as link, beam, plate, shell or volume. All elements are described by nodes which are connected with even or curved lines. Thus, the geometry of each element is known. The displacement within the elements is calculated via approximated relations depending on the nodes' displacements. The node displacement of neighbouring elements is equal. So the force equilibrium is reached at each node and the system of equations is given for the nodes' displacement. The dimension of the system of equation corresponds to the number of nodes' displacement. By solving the system of equation, the nodes' displacement is achieved depending on the loading conditions. Other parameters, e.g. stresses can now be calculated by the displacement of nodes. 
The finite element method is also applicable for hydrodynamic or electronic problems. If a question can be formulated with linear or partial differential equations it can be solved with the finite element method (see Lehmann [34]).

There are two different time integration methods within FEM. Depending on the problem either an implicit or an explicit time integration is used. The implicit method first calculates the global equilibrium for each integration step and then the local element variables. Equation (2.15) is solved by converting the stiffness matrix to gain the displacement vector:

$$
\begin{aligned}
& {[M]\{\ddot{x}\}+[K]\{x\}=\{f\} } \\
\Rightarrow & \{x\}=[K]^{-1} \cdot(\{f\}-[M]\{\ddot{x}\}) \\
& {[M]: \text { mass matrix } } \\
& {[K]: \text { stiffness matrix } } \\
& \{\ddot{x}\}: \text { acceleration vector } \\
& \{x\}: \text { displacement vector } \\
& \{f\}: \text { external forces vector }
\end{aligned}
$$

The matrices are calculated iteratively. The integration is often done with the Newark method. In every integration step the equilibrium is determined. Therefore, the implicit method is best applicable to:

- linear problems

- static problems (change of external forces is small)

The explicit method (Euler forward time integration) directly calculates the global variables without determining the global equilibrium. In each time step the following equation is solved with the central difference procedure:

$$
\begin{gathered}
{[M]\{\ddot{x}\}=\{f\}-[K]\{x\}} \\
\Rightarrow\{\ddot{x}\}=[M]^{-1} \cdot(\{f\}-[K]\{x\})
\end{gathered}
$$

In the explicit method, criteria are needed to guarantee the correctness and the stability of the calculation because it does not have any convergence criterion. The Courant criterion is taken. It defines the maximal calculation time step which is equal to the time that an acoustic wave needs to cross the smallest element. In each calculation step the calculation time step size is determined. Thus, the calculation time step has a size of 1 to 
$10 \mathrm{~ms}$. If a simulation of a problem takes $10 \mathrm{~s}$ real time, the calculation needs 10 to 100 thousand time steps.

The explicit time integration method is recommended for the following problems:

- dynamic problems (acceleration)

- large deformation and contact (stiffness changes)

- rigid body motion (stiffness matrix can be solved)

- ultimate strength, snap-through buckling, crippling (external forces change due to change in stiffness)

- plasticity (large non-linearities)

If structural response with extensive plasticity is simulated, as is the case for stranding, FE codes offer advantages compared to analytical techniques. The modelling allows the description of complicated geometries. The material models are far more realistic than the material models incorporated in analytical techniques. It is not necessary to assume the failure modes of the structural components as needed for using upper and lower bond theorems to predict the collapse strength of structures. However, the use of FEM is linked to uncertainties. The results depend on the selection of mesh and element types, on material deformation and failure modes.

Most of this work is based on the application of FEM. All static/linear problems (see section 3 and 5) within this work are solved with an implicit FE method. The program $A N S Y S$ and LS-DYNA are used in its latest versions. The results presented in section 4 are calculated with the explicit version of $L S-D Y N A$. 



\section{Parameter study}

A simple method is presented for calculating the total bending moment, shear force and heeling angle due to grounding for any ship depending on the load case, the grounding point/area and the surface drawdown. The ship form is not simplified. The hydrostatic forces are calculated exactly. Due to its specific form, it is not possible to calculate the new distribution by hand and therefore computer tools are used.

The purpose is to find the critical combinations of stranding point/area, surface drawdown and load case, which lead to an exceedance of the global bending moment and shear force required by classification societies and/or instability in the final position. Furthermore, the value of the additional forces and moments caused by stranding are determined and the sections at risk are identified. By way of example, this method is applied to a container vessel.

\subsection{The proposed method}

It is assumed that the ship structure does not suffer important damage between the initial contact with the ground and the final stranding position (see section 2.1). This assumption has also been made by Lehmann [35], Pedersen [52], Reich and Röhr [62] and Östergaard et al. [47].

The hull behaves predominantly as a rigid body. Therefore, the immersion of bow and stern, when the ultimate resistance of the girder is reached is neglected.

Grounding of the vessel at certain points/areas including surface drawdowns due to receding tide is simulated with the ship design program $e 4$ (Krüger [31]). Then, the resulting load forces and water pressure are applied to a beam modelled in the finite element program ANSYS 14.0 (Ansys [4]). A FE program is chosen because later the beam is partly replaced by a shell model (see section 4 ). As a result the total bending moment and the total shear force over the ship length caused by a grounding incident and changes in water level are gained for a predefined load case. Those data are transferred to a mathematical optimisation method. It is possible to predict all critical combinations of the various parameters at once although only few grounding incidents are calculated via ANSYS. Results in between the cases that are calculated in ANSYS can be determined due to approximation and interpolation methods.

\subsubsection{Grounding calculation in e4}

In $e 4$, the ship form is described by a series of curves, which are determined by points and an interpolation code. The interpolation code is a two-dimensional cubic parametric 
spline. The floating condition is calculated by the theory of stability (see Söding [75]). The vessel is considered to be rigid.

First the user chooses the load case. In the grounding subroutine the user defines the position of grounding, surface drawdown and whether the trim and the heel are fixed or free. Here, the trim and the heel are always set free. They are determined by the theory of stability, so that the moments relative to the grounding location are equalised (see Krüger [32]). The subroutine generates a sheet of hydrostatic tables. The relevant data are the trim, the displacement and the reaction force as a function of surface drawdown at the grounding point.

For each floating condition, a sectional area curve is generated. The sectional area curves are generated on the basis of volumetric calculations. Each so-called 'calculation frame' is described as a function. The function is integrated to achieve the sectional area. The areas between the frames are interpolated. The buoyancy at the centre line is converted into a line load distribution by integration of the sectional area curve.

The weight distribution is also required for the FE calculations. e4 gives all relevant weights as a line load distribution at the centre line depending on the load case.

\subsubsection{Simple model}

The total bending moments and vertical forces due to the grounding cases are calculated in ANSYS and exported as data files. The ship is modelled as a beam. The element type chosen is based on the Timoshenko beam theory. The forces resulting from weight and buoyancy are applied as line loads onto the beam elements.

The line load distribution from the floating condition generated in $e 4$ is in a ship-fixed coordinate system. For a correct FE calculation, the distribution should be in aground-fixed reference system. A test with a pontoon $\left(\mathrm{L}=100 \mathrm{~m}, \mathrm{~B}=1 \mathrm{~m}, \mathrm{~T}=20 \mathrm{~m}\right.$, trim angle $=5^{\circ}$ ) reveals that the difference of the buoyancy distribution in a ship-fixed and a ground-fixed system is marginal. A difference in the distributions only occurs at the first metre of one end. After the first metre, the difference is less than $0.4 \%$. For a real ship, the difference is insignificant since ships have proportionally the least buoyancy at the bow and the stern. The correct moment of inertia of the beam is in fact not required for the method because only the moments and forces are examined. But for later simulation the displacement is needed. Therefore the correct moments of inertia are taken from POSEIDON (see section 4). For the calculation with the grounding point, five degrees of freedom are fixed at the grounding point. Only the rotation around y is free. The grounding area is modelled with springs to simulate an elastic foundation. All springs have the same stiffness and the equilibrium position is automatically achieved. The stiffness does not influence the courses of the moments or shear forces. Therefore, the stiffness of the springs is chosen to be one. A drawing of the beam and its boundaries is presented in figure 3.1 for each grounding case. The coordinate system is plotted and the vessel is shown in light grey. 


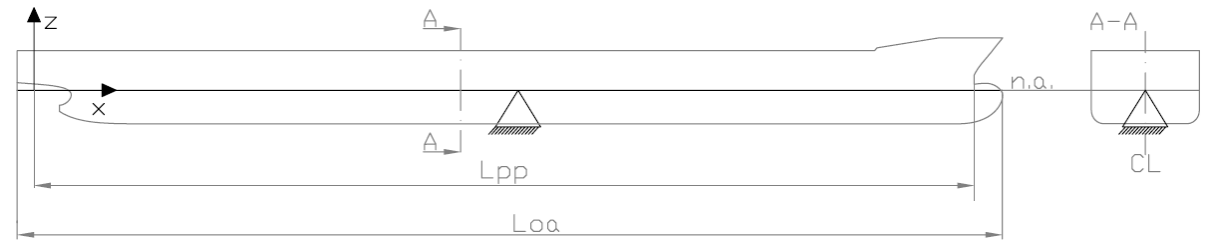

(a) Cases A1, B1 and C1

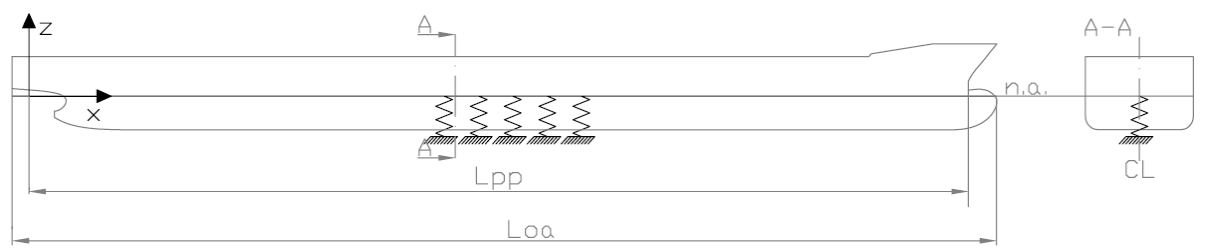

(b) Case A2

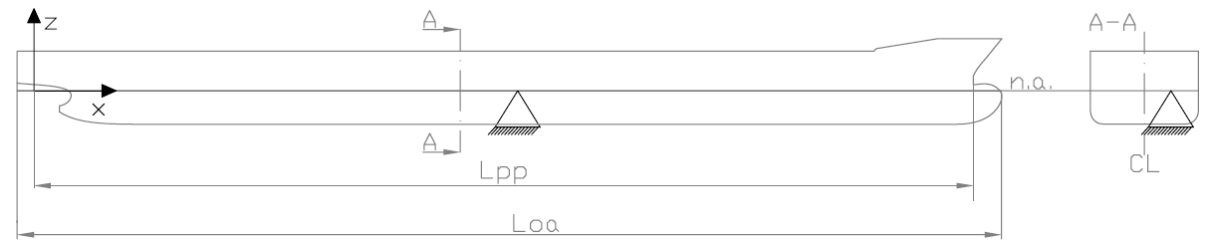

(c) Case A1y

Figure 3.1.: Beam (idealised ship).

\subsubsection{Dimensions and load cases}

The container vessel, already described in section 2 , is chosen to illustrate this method. The relevant dimensions of the Postpanmax are given in table 2.4. Postpanmax is fully inserted in $e 4$ and in POSEIDON [17].

The effects of different loading conditions are examined. Thus, three load cases are considered: the arrival (A), the ballast (B) and the departure case (C). All load cases are typical for estuary voyage.

In the arrival case, the ship has its design draugt. Figure 3.2 shows the weight distribution, shear force and bending moment calculated for this load case. The figures for case B and $\mathrm{C}$ are in appendix A.2.

During the ballast voyage, the ship's draugt is $6.26 \mathrm{~m}$. This load case has the largest bending moment and therefore defines the moments for the main frame design.

The vessel floats on the design draugt while departing from a port. The still water bending moment is the smallest compared with the other two load cases.

Load case A is chosen for most calculations because its still water bending moment lies between the one of load case B and C.

Furthermore, the effect of tank flooding on the bending moment and the shear force is examined. It is assumed that the double bottom fractures close to the stranding point during receding tide. The structure fractures due to the additional forces and moments during stranding. Water floods into the empty or partly filled tanks of the double bottom.

Table 3.1 gives an overview of the calculated cases. Most cases are calculated for different coordinates of stranding points or area. The cases A2, A1y, B1, C1 and A1f are compared with case A1. 


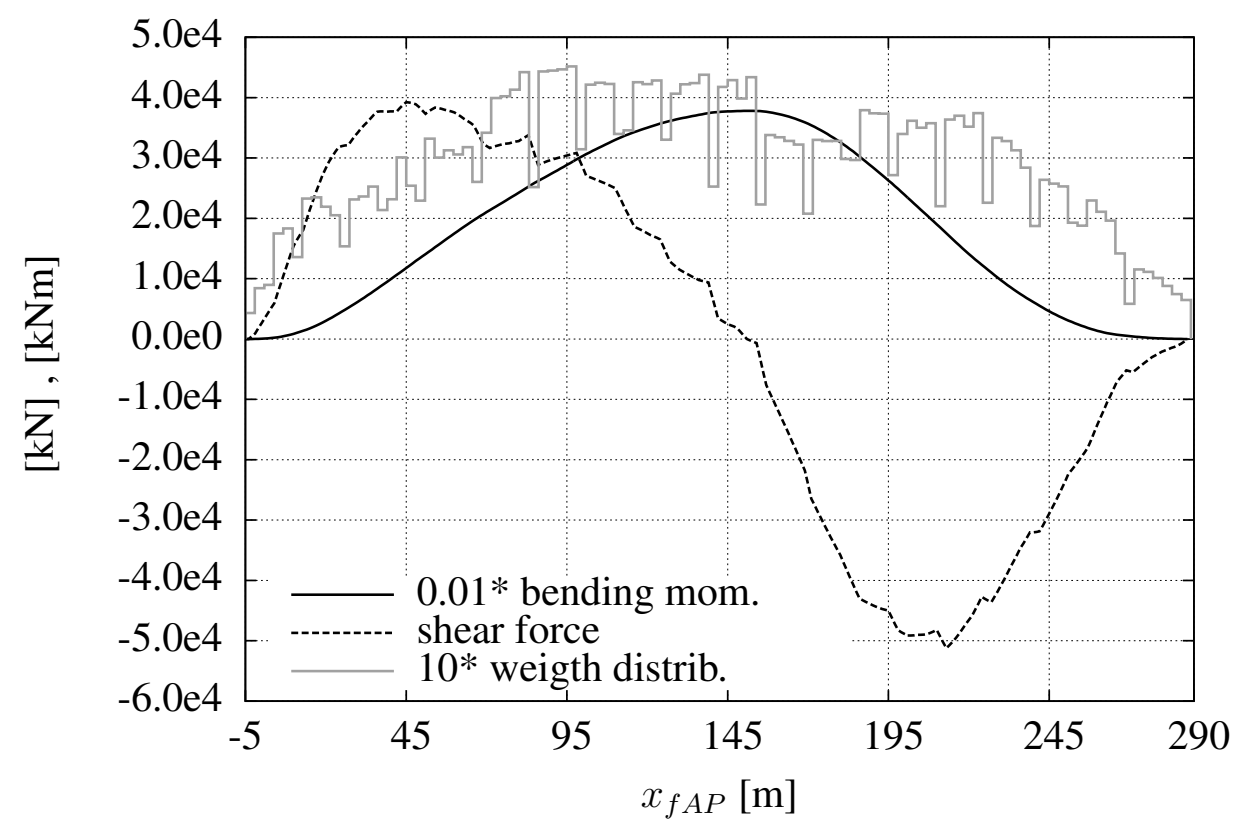

Figure 3.2.: Load case A: weight distribution, shear force and still water bending moment.

Table 3.1.: Stranding cases.

\begin{tabular}{lrrrrr}
\hline case & load case & point/area & coo. $\mathbf{x}[\mathbf{m}]$ & coo. $\mathbf{y}[\mathbf{m}]$ & flooding \\
\hline A1 & A & point & $5,25, \ldots, 265,285$ & 0 & no \\
A2 & A & area & 145 & 0 & no \\
A1y & A & point & 145 & 8 & no \\
B1 & B & point & $65,145,265$ & 0 & no \\
C1 & C & point & $65,145,265$ & 0 & no \\
A1f & A & point & $70,145,250$ & 0 & yes \\
\hline
\end{tabular}

In each stranding case, the water level is changed. The initial floating condition is calculated (no grounding, no tide) and then the surface is drawn down in $1 \mathrm{~m}$ steps to a total change of $5 \mathrm{~m}$. 5 to $6 \mathrm{~m}$ of tidal range are found e.g. at the water mouth of the river Seine.

\subsubsection{Optimisation method}

Optimisation is applied to find the best approximation to the above calculated functions of bending moment and shear force by keeping the computational effort as low as possible. The main purpose is to provide one graphical chart in which all critical combinations of the parameter $x_{f A P}$ (= ship length coordinate), $x_{\text {cont }}$ (= stranding point), $s_{d}$ (= receding tide level) can be shown for the bending moments, the shear forces or even both. Due to interpolation all parameter combinations are available even if only some incidents (combinations of $x_{f A P}, x_{\text {cont }}$ and $s_{d}$ ) are calculated with ANSYS. 
The graphical chart is assessed by limit curve points for different $s_{d}$-values as schematically shown in figure 3.3 . In the resulting charts the points are not connected with lines. In figure 3.3 the points are connected by dashed lines for a better overview. In the resulting chart there are enough points so that limit curves can be recognised. The limit curve points enclose areas where the class permissible bending moments $M_{T \text { pos } \backslash \text { neg }}(n)$ or the class permissible shear forces $Q_{T}$ pos $\backslash$ neg $(n)$ are exceeded due to critical combinations of $\left(x_{f A P}, x_{\text {cont }}, s_{d}\right)$. The class permissible bending moments and shear forces are determined according to the rules of Germanischer Lloyd. They are the sum of class permissible still water and vertical wave bending moments/shear forces.

In the following the optimisation method is exemplarily explained for the bending moments. For further details see Schenke [66].

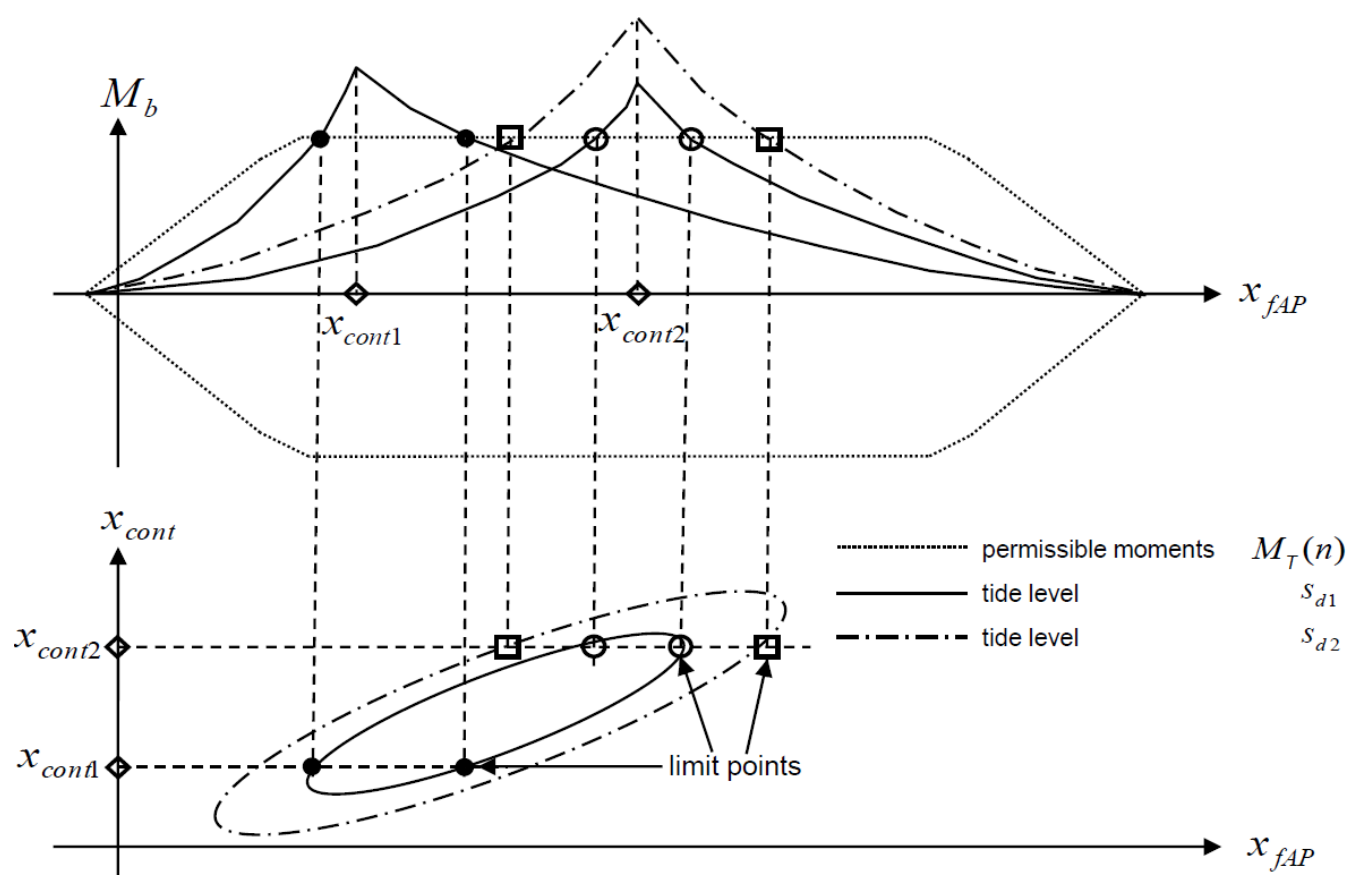

Figure 3.3.: Schematic diagram of limit curve points.

The bending moment curves calculated with ANSYS are summed up in a data field $M_{b}(n, k, m)$. The continuous ship coordinates $\left(x_{f A P}, x_{c o n t}, s_{d}\right)$ are transformed into discrete field coordinates $(n, k, m)$. Approximation and interpolation methods enable results for any coordinate even between the discrete points $(n, k, m)$. The class permissible bending moments $M_{T}(n)$ are attached to the coordinates $(n, k, m)$ of the data field. $M_{b}(n, k, m)$ and $M_{T}(n)$ are the objects to be analysed.

Limit curve points are described by the set $G=\left\{P_{G i}\right\}$ where $P_{G i}$ are discrete points of a limit curve.

Further aspects of the optimisation method to identify critical combinations are:

- Identify local maximum exceedance within one set of limiting curve points $(m=$ const) and its position. 
- Find limits of critical sub-intervals $I_{C r i t n \backslash k} \in G=\left\{P_{G i}\right\}$, if $m, n=$ const or $m, k=$ const.

- Obtain the global maximum exceedances within the critical sub-intervals. These may coincide with the local maximum exceedance.

The problem at hand is a combinatorial optimisation problem because the permissible range $S$ is finite. The data field $M_{b}(n, k, m)$ contains of discrete functional values and is limited by parameter bounds. The field coordinates $n, k$ have functional values only in a bounded interval that is limited by the ship length $\ell$. The parameter $m$ is bounded by the draught of the ship. In particular the parameter bounds are defined as:

$$
1 \leq n \leq \ell ; \quad 1 \leq k \leq b ; \quad 1 \leq m \leq t \quad \text { with } n, k, m \in \mathbb{N}
$$

These parameter bounds finally define the permissible range $S$. The parameters $b$ and $t$ depend on the resolution configuration $\left(w_{k}, w_{m}\right) . w_{k}$ and $w_{m}$ are the number of additional data generated between two known data points obtained from the ANSYS calculation. $w_{k}$ additional data sets are calculated between two known bending moment curves $\left(w_{m}\right.$ accordingly for the surface drawdown).

$M_{b}(n, k, m)$ and $M_{\text {diff }}(n, k, m)$ are nonlinear and limit curve points are not existent at the bounds of the data field. Therefore, the optimisation problems can be solved via the so-called simplex-method without the Karush-Kuhn-Tucker requirement (see Jungnickel [28] and Großmann and Terno [21]).

To solve the above given aspects different optimisation problems have to be formulated. At first the following case-by-case analysis is needed, because every point $(n, m, k)$ is related to two allowable moments $\left(M_{T \text { pos }}(n)\right.$ and $\left.M_{T \text { neg }}(n)\right)$ :

$$
\begin{array}{r}
\left|M_{b}(n, k, m)-M_{\text {T pos }}(n)\right|<\left|M_{b}(n, k, m)-M_{\text {T neg }}(n)\right| \\
\Rightarrow \quad M_{\text {diff }}(n, k, m)=M_{\text {T pos }}(n)-M_{b}(n, k, m) \\
\text { else } M_{\text {diff }}(n, k, m)=M_{b}(n, k, m)-M_{\text {T neg }}(n)
\end{array}
$$

Thereby the first optimisation problem is:

$$
\begin{aligned}
& \mid M_{\text {diff }}(n, k=\text { const, } m=\text { const }) \mid \stackrel{!}{=} \text { max with } \quad M_{\text {diff }}<0 \\
& \mid M_{\text {diff }}(n=\text { const }, k, m=\text { const }) \mid \stackrel{!}{=} \max \text { with } \quad M_{\text {diff }}<0
\end{aligned}
$$

To get the local maximum exceedance of $M_{T}(n)$ within the limiting curve, equation (3.4) is solved:

$$
M_{\text {diff }}(n, k, m=\text { const }) \stackrel{!}{=} \text { min with } \quad M_{\text {diff }}<0
$$

The problem formulation for critical sub-intervals $I_{C r i t n \backslash k}$ results for equation (3.3) in: 


$$
\begin{aligned}
& M_{\text {diff }}(n=\text { const }, k, m=\text { const }) \stackrel{!}{=} \text { min with } \quad M_{\text {diff }}<0 \\
& M_{\text {diff }}(n, k=\text { const, } m=\text { const }) \stackrel{!}{=} \text { min with } \quad M_{\text {diff }}<0
\end{aligned}
$$

Global maximum exceedance within the critical sub-intervals can be calculated by equation (3.4), if $n$ or $k$ are also constant.

All points are defined as a set by one integer in $G 0(n, k, m)$. Hence, the combinational optimisation problem is transformed into an integer problem appropriate to the classification of $S$ :

$$
\begin{aligned}
& G 0(n, k, m=\text { const })=-1, \text { if } P=(n, k, m=\text { const })^{T} \text { fulfils equation (3.3) } \\
& G 0(n, k, m=\text { const })=-2, \text { if } P=(n, k, m=\text { const })^{T} \text { fulfils equation (3.4) } \\
& G 0(n, k, m=\text { const })=-0, \text { else }
\end{aligned}
$$

Local and global approximation of the discrete function is implemented to calculate data between the known discrete points. Local interpolation is used between two discrete points with interpolating polynomials and approximation of the course of function around a discrete expansion point with Taylor polynomials. The interpolation does not reproduce the curvature. A Taylor polynomial applies only to the expansion point but any derivatives of the output function can be reproduced. Since the output function is in discrete form, the derivatives are approximated with a difference quotient within the expansion point. To globally approximate additional bending moments in $m$-direction linear interpolation is chosen, whereas in $k$-direction an equalisation calculus is used.

The optimisation method is implemented with MATLAB [41]. The program flow of the optimisation is shown in figure 3.4. The flow charts of the mentioned functions are given in appendix A.1

$M \_$interpol (see figure A.1) defines the data field $M_{b}(n, k, m)$ in the desired resolution. $M \_$diff_interpol (see figure A.2) attaches the allowable moments $\left(M_{T}\right)$ to the data field $M_{b}(n, k, m)$ and calculates the difference $M_{\text {diff }}(n, k, m)$. Limit points have a value of 0 within the field $M_{\text {diff }}(n, k, m)$. G0_interpol (see figure A.3) characterises the data $M_{\text {diff }}(n, k, m)$ by assigning integers. One integer in $G 0(n, k, m)$ stands for a defined quality. The limit curve points from $G 0(n, k, m)$ are directly plotted as single points. If the resolution $w_{k}$ is sufficient one sees curves.

Crit_Max_x_fAP_xcont (see figure A.4) calculates local maximum exceedance of $M_{T}$ for $M_{\text {diff }}(n, k, m=\mathrm{const})$. Crit_Interval_x_fAP (see figure A.5) and Crit_Interval_xcont give the limit of the critical intervals $I_{C r i t n}$ or $I_{C r i t k}$. CritMax_x_fAP and CritMax_xcont calculates the maximum exceedance within these intervals.

An additional tool of the above described optimisation method is to superimpose critical bending moments and shear forces. In one diagram one can see which parameter combinations lead to the most critical combination of bending moment and shear force. For that purpose a relative difference is introduced to get the percentage exceedance of permissible $M_{T}$ and $Q_{T}$ : 


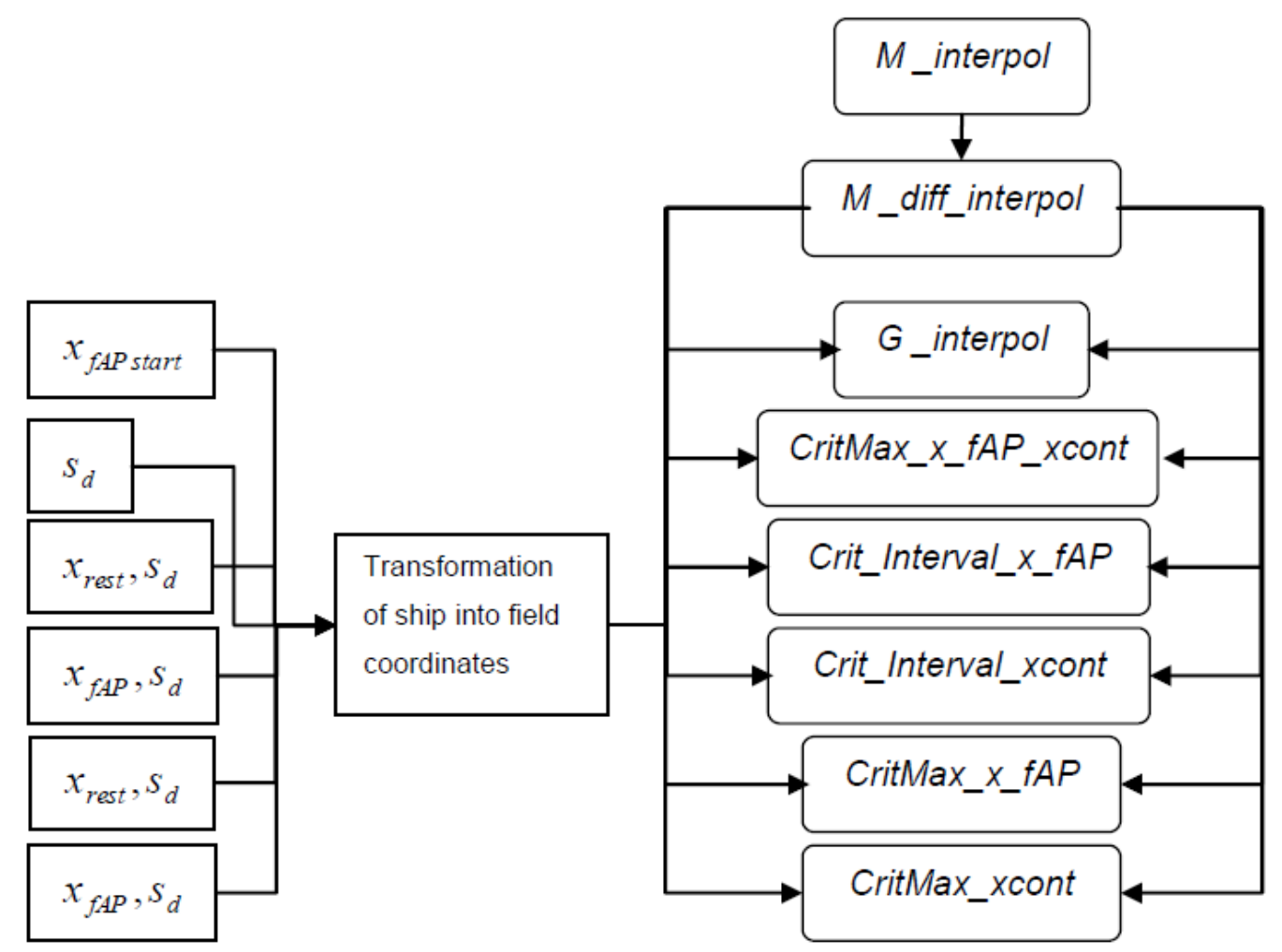

Figure 3.4.: Flow chart of the optimisation program.

$$
\begin{aligned}
& M_{\text {diffrel }}(n, k, m)=\left|\frac{M_{\text {diff }}(n, k, m)}{M_{\text {Tact }}(n)}\right| \text { if } M_{\text {diff }}(n, k, m)<0 \\
& M_{\text {diffrel }}(n, k, m)=0 \text { else } \\
& Q_{\text {diffrel }}(n, k, m)=\left|\frac{Q_{\text {diff }}(n, k, m)}{Q_{\text {Tact }}(n)}\right| \text { if } Q_{\text {diff }}(n, k, m)<0 \\
& Q_{\text {diffrel }}(n, k, m)=0 \text { else }
\end{aligned}
$$

The index act is the active positive or negative value of $M_{T \text { pos } \backslash \text { neg }}(n)$ or $Q_{T \text { pos } \backslash \text { neg }}(n)$ within $n$ (see also equation (3.2)). The dimensionless values can now be superimposed to one field $E_{M Q}(n, k, m=$ const $)=E_{M Q}(n, k)$ with the weighting coefficient $\alpha$.

$$
E_{M Q}(n, k)=\alpha \cdot M_{d i f f r e l}(n, k)+(1-\alpha) \cdot Q_{d i f f r e l}(n, k)
$$

For a simple use of all tools a graphical user interface with certain defaults is implemented. The GUI is independent of the input data which are chosen first. The step size of the surface drawdown $\left(w_{m}\right)$ can be switched from $0.1 \mathrm{~m}$ to $0.5 \mathrm{~m}$. The step size in ship length direction $\left(w_{k}\right)$ is $1 \mathrm{~m}$. The user chooses between results for moments or shear forces and can plot the limit curve points. To find limits of critical sub-intervals and global exceedance within these sub-intervals one defines a constant value either $x_{f A P}$ or $x_{\text {cont }}$. Now for any chosen tide level a result is gained. The result is given as value and is marked in the plot of the limit curve points. Furthermore, the user can get the maximum 
overstepping of $M_{T}$ or $Q_{T}$ for a constant tide level and can produce a plot of the total bending moment or of the shear force over the ship's length for any tide level and any stranding point within the limits $\left(s_{d}=0-5 \mathrm{~m}, x_{\text {cont }}=-5-289 \mathrm{~m}\right)$. Figure A.6 in appendix A shows the interface with results for $s_{d}=2 \mathrm{~m}$ and $x_{f A P}=x_{\text {cont }}=145 \mathrm{~m}$.

\subsection{Verification of the simple method}

The stranding case A1 (load case A, various stranding points located at centre line, without flooding) is also calculated with existing formulas of Lehmann [35], Pedersen [52] and Östergaard et al. [47] to verify the method. All three make the same assumptions as assumed for the proposed method: no damage during first contact with the ground and the final stranding position and the hull behaves as a rigid body (see section 2).

The additional bending moment due to grounding is calculated at three points $(65,145$ and $265 \mathrm{~m} \mathrm{AP}$ ) and again for $5 \mathrm{~m}$ of tide levels. Therefore, a total of 15 moments with each formula are assessed and compared with the results of the described method.

In order to achieve more clarity, the additional bending moments due to grounding are classified as follows:

- $M_{Z}(\mathrm{x})$ : additional bending moment at all length metres after Zipfel.

- $M_{L}$ : additional bending moment at $143 \mathrm{~m} \mathrm{AP}$ after Lehmann [35] with $c_{W p}=0.8$ and $c_{M}=0.83$.

- $M_{O}(\mathrm{x})$ : additional bending moment at all length metres after Östergaard et al. [47], multiplied by the coefficient of water plane $c_{W p}=0.8$.

- $M_{P}(\mathrm{x})$ : additional bending moment at all length metres after Pedersen [52], multiplied by the coefficient of water plane $c_{W p}=0.8$, the longitudinal centre of floatation $L C F$ is taken from the hydrostatic tables (see subsection 3.1.1).

To attain $M_{Z}(\mathrm{x})$, the still water bending moment from load case A (see figure 3.1) is subtracted from the calculated total bending moment due to the relevant grounding position.

The formula of Lehmann [35] gives only results at the position $L_{p p} / 2=143 \mathrm{~m}$ AP. The required coefficients in the formula are for a block coefficient of $c_{B}=0.7$.

The formulas of Östergaard et al. [47] and Pedersen [52] are valid for a pontoon or a square-shaped ship. The formulas are multiplied by the same water plane coefficient as required for $M_{L}$ to partly include the effect of the real water plane. The qualitative results of Pedersen [52] mostly depend on the location of $L C F$.

In figures 3.5-3.7, the additional bending moments $M_{Z}(x), M_{O}(x)$ and $M_{L}$ are plotted for three water levels $(1,3$ and $5 \mathrm{~m})$. The moments $M_{P}(x)$ are not shown to achieve more clearness. They are qualitatively equal to $M_{O}(x)$ and their maximum values lie in between $M_{L}$ and $M_{O}(143 \mathrm{~m})$. Table 3.2 gives the average deviation of $M_{L}, M_{P}(143 \mathrm{~m})$ and $M_{O}(143 \mathrm{~m})$ to $M_{Z}(143 \mathrm{~m})$. The percentage differences of the moments resulting from each water level are averaged for the considered stranding point. 
Table 3.2.: Average deviation of $M_{L}$ and $M_{O}(143 \mathrm{~m})$ to $M_{Z}(143 \mathrm{~m})$.

\begin{tabular}{lrrr}
\hline $\mathbf{x}_{\text {cont }}$ & $\mathbf{M}_{\mathbf{L}}[\%]$ & $\mathbf{M}_{\mathbf{P}}(\mathbf{1 4 3} \mathbf{~ m})[\%]$ & $\mathbf{M}_{\mathbf{O}}(\mathbf{1 4 3} \mathbf{m})[\%]$ \\
\hline $\mathbf{6 5} \mathbf{~ m}$ & 48.85 & 58.90 & 66.08 \\
$\mathbf{1 4 5} \mathbf{~ m}$ & 7.65 & 24.17 & 40.22 \\
$\mathbf{2 6 5} \mathbf{~ m}$ & 5.96 & 8.15 & 9.42 \\
\hline
\end{tabular}

For stranding in the midship or the bow part of the vessel the moments after Lehmann [35] do not differ much from the moments achieved by the method presented here. The formula after Lehmann [35] is a conservative estimation for these cases. If the vessel strands in the aft part the moments $M_{L}$ are different to $M_{Z}(\mathrm{x}=143 \mathrm{~m})$.

Östergaard et al. [47] and Pedersen [52] moments take good courses of the moment for running aground in the forward part (see figure 3.7). At the stranding point the moments are almost the same, whereas amidships at $143 \mathrm{~m}$ a deviation is seen. In the other two cases, the absolute value of the moments $M_{O}(\mathrm{x})$ and $M_{P}(\mathrm{x})$ at the stranding point are explicitly higher than $M_{Z}(\mathrm{x})$. For grounding at $145 \mathrm{~m}$ the curve of $M_{O}(\mathrm{x})$ equals the one of $M_{Z}(\mathrm{x})$ (see figure 3.6).

Because all three formulas do not consider the real ship form the results differ from the method presented here. The real buoyancy distribution of a slender ship is not included. In particular, the formulas do not produce good estimations for grounding in the aft part of slender ships. Then the bow immerses and the vessels' bow produces less over-plus of buoyancy than assumed by Lehmann [35], Östergaard et al. [47] and Pedersen [52]. The coefficient of water plane $c_{W p}$ does not adequately considers the bow shape.

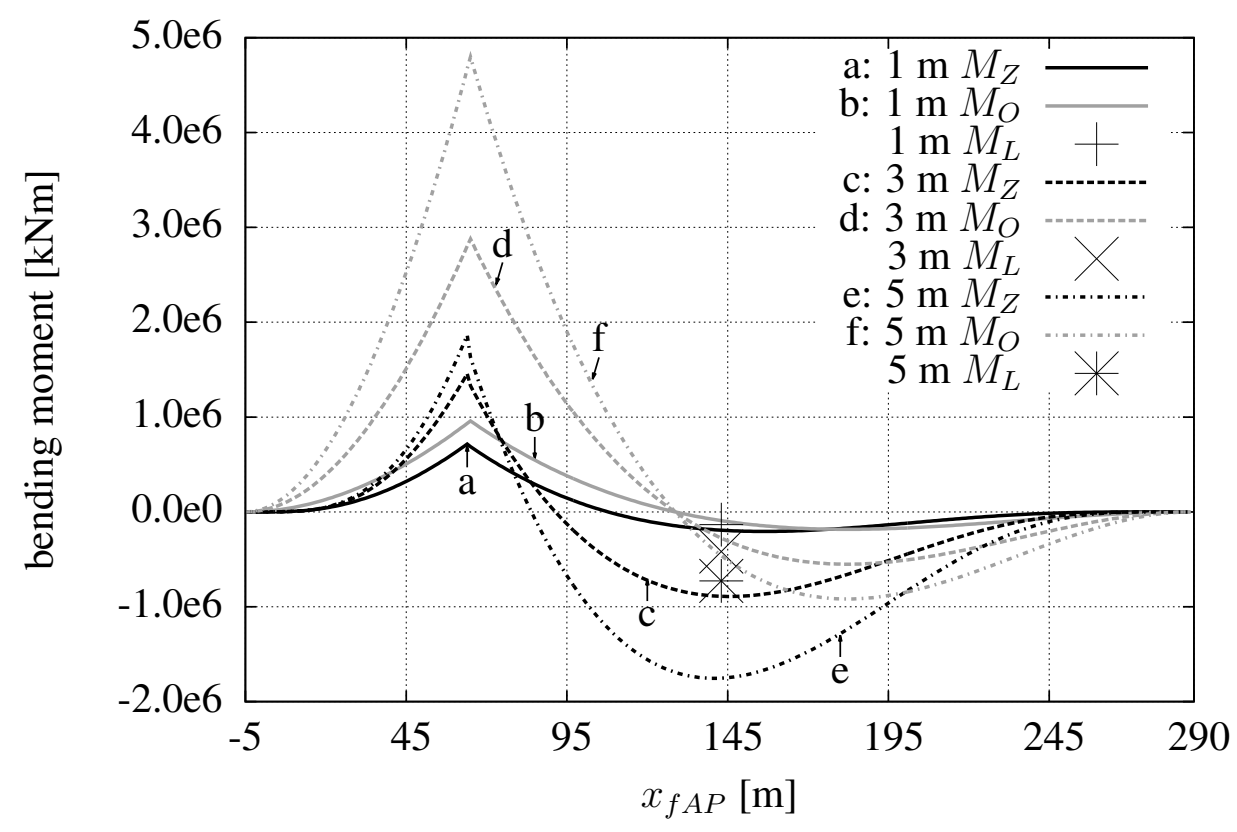

Figure 3.5.: Additional bending moments due to stranding at $65 \mathrm{~m}$ AP. 


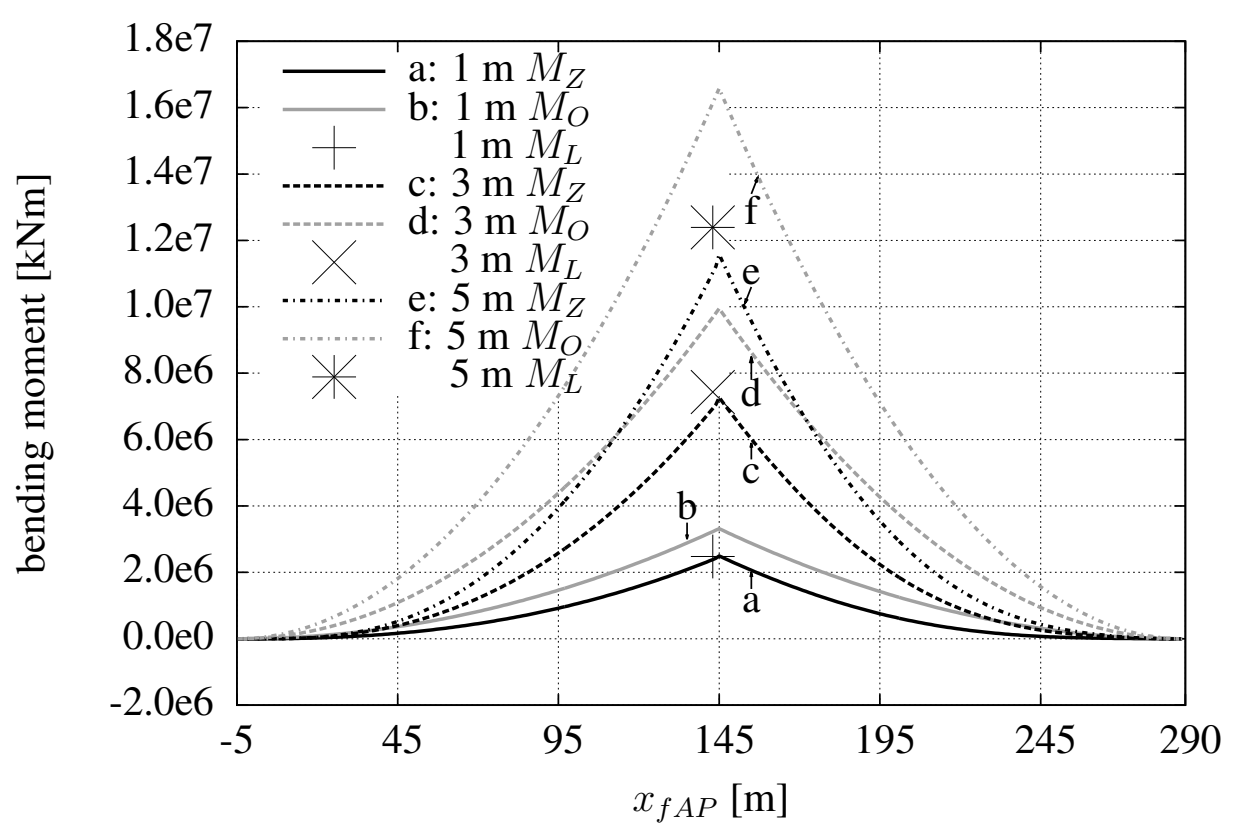

Figure 3.6.: Additional bending moments due to stranding at $145 \mathrm{~m}$ AP.

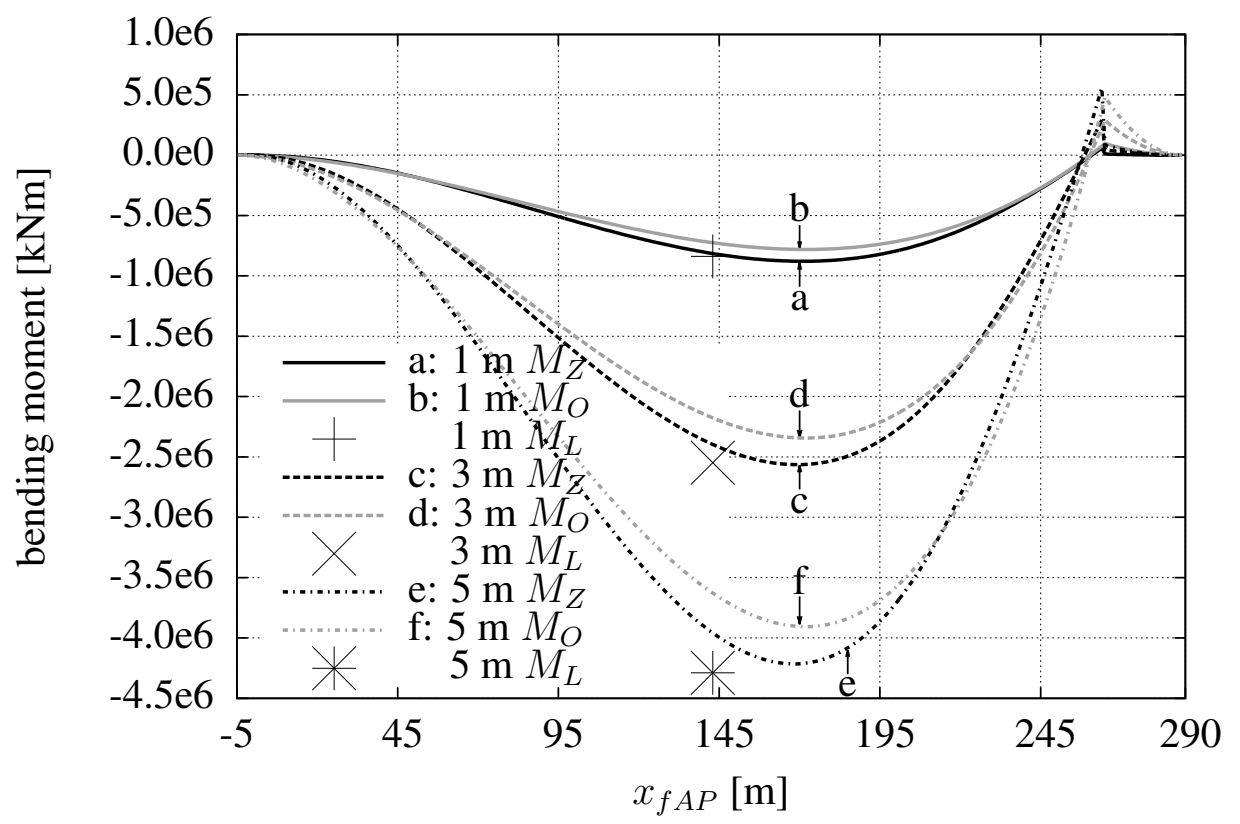

Figure 3.7.: Additional bending moments due to stranding at $265 \mathrm{~m}$ AP.

If the stern immerses all methods give similar results. Thus, for this case (stranding in the fore body and midship part) the ship form can be simplified. But if the bow immerses one needs to take the real ship form into account.

Similar results to the method presented here could be achieved for accidents amidships by combining the formula of Lehmann [35] and Östergaard et al. [47] (see figure 3.6). 


\subsection{Calculation results}

The results of the ANSYS calculation and the optimisation method are presented in the following. The ANSYS results provide the input data for the optimisation method. Therefore the quality of the limit curves depends on the amount of calculated cases in ANSYS.

For selected grounding positions the total bending moments and shear forces are plotted over the ship length. Thereby, the class permissible bending moments $\left(M_{T}\right)$ and shear forces $\left(Q_{T}\right)$ are always plotted with a grey line. The $M_{T}$ respectively shear forces include the reserve between the still water and the seagoing conditions of the structure. The grounded vessel is probably not subjected to wave loads (see section 2), because soft grounding happens close to the coast during estuary voyage. So, if the curves are exceeded, the structure will sustain severe damage.

\subsubsection{Stranding case $\mathbf{A} 1$}

Stranding case A1 is calculated with load case A, without flooded tanks and at 15 stranding points that are located at the centre line. The positions are from 5 to $285 \mathrm{~m}$ AP in $20 \mathrm{~m}$ steps. The results of the optimisation method are shown in figure 3.8 and 3.9. In figures 3.8 and 3.9 the limit curve points, the critical intervals of $s_{d}=2,3,4$ and $5 \mathrm{~m}$ for $x_{f A P}=x_{\text {cont }}=145 \mathrm{~m}$, the maximum exceedance within the critical interval (red cross) and the maximum overstepping of $M_{T}$ for a tide level of $2 \mathrm{~m}$ are plotted (box only for the moments). The absolute values of the last three listed results for the moments can also be seen in the interface (see figure A.6). Figures 3.10-3.12 show by way of example the bending moments and shear forces when the vessel rests on the ground at 65, 145 and 265 $\mathrm{m}$ from AP for each tide level.

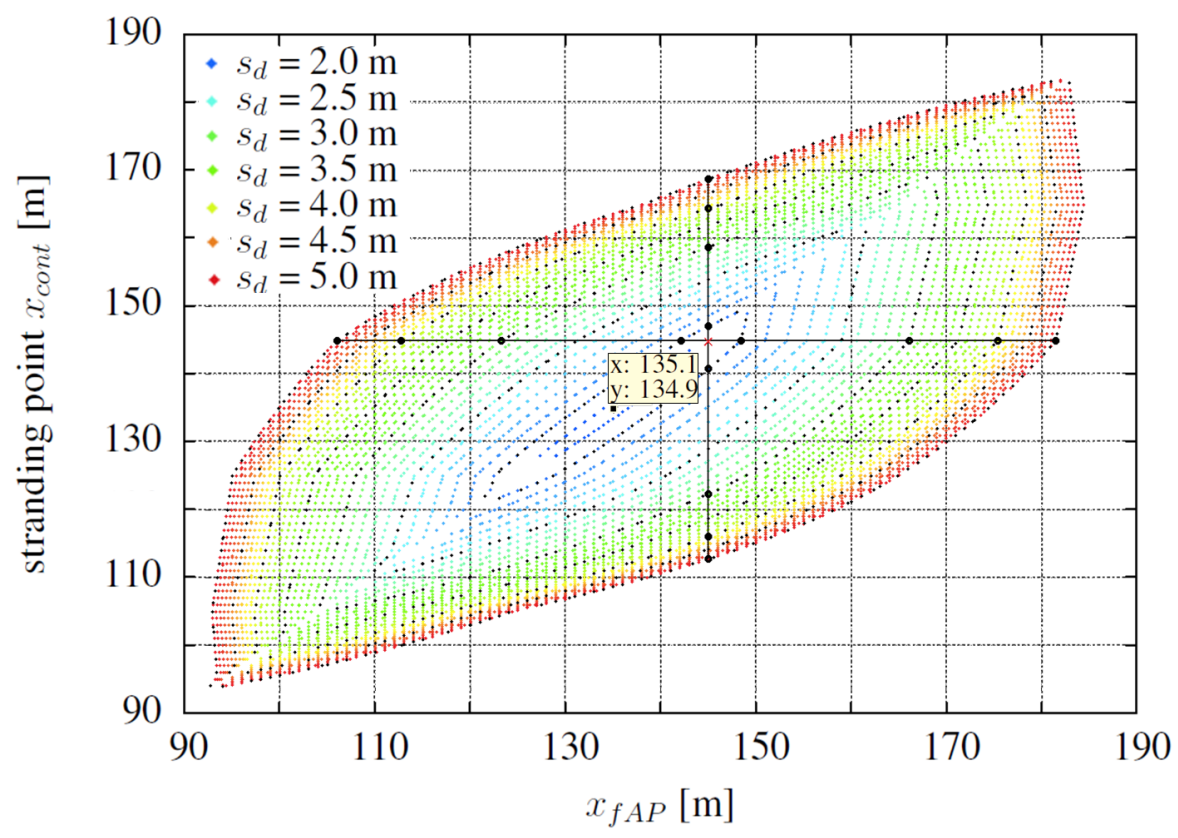

Figure 3.8.: Case A1: limit curve points of bending moments. 


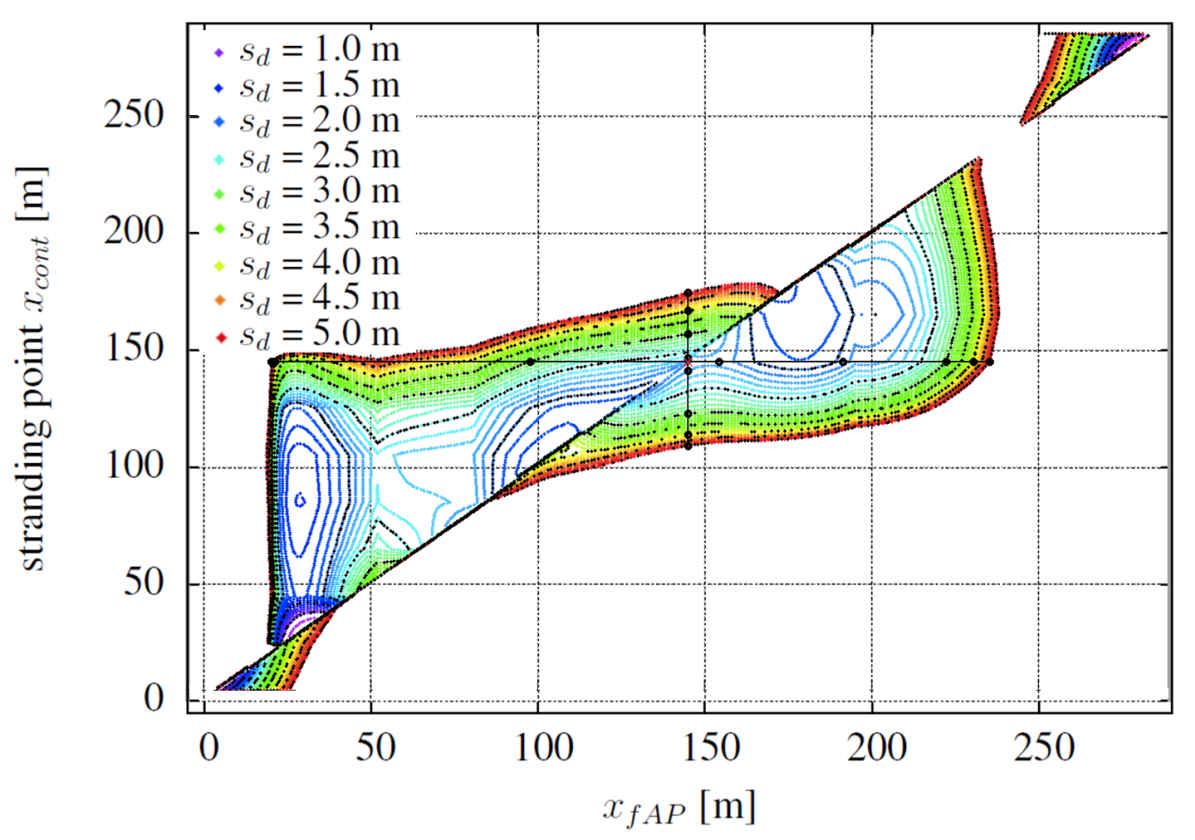

Figure 3.9.: Case A1: limit curve points of shear forces.

The exceedance of the class permissible $M_{T}$ always occurs around the contact point. The critical stranding points for the bending moment are amidship between $94 \mathrm{~m}$ and 184 $\mathrm{m}$ from AP (see figures 3.8 and 3.11). Running aground between 99 and $179 \mathrm{~m} \mathrm{AP}$ is critical for $4 \mathrm{~m}$ of receding tide. $3 \mathrm{~m}$ of surface drawdown lead to overstepping of $M_{T}$ for grounding between 106 and $169 \mathrm{~m}$ AP. If the vessel stands between 122 and $149 \mathrm{~m}$ AP all tide levels higher than $2 \mathrm{~m}$ are critical.

The maximum bending moments for fixed tide levels always have the same coordinates $x_{f A P}=x_{\text {cont }}$ and are located amidship. For a water level reduction of $s_{d}=2 \mathrm{~m}$ the maximum is reached for $x_{f A P}=x_{\text {cont }}=135 \mathrm{~m}\left(s_{d}=3 \mathrm{~m}: x_{f A P}=x_{\text {cont }}=140 \mathrm{~m}, s_{d}=4 \mathrm{~m}\right.$ : $x_{f A P}=x_{\text {cont }}=143 \mathrm{~m}$ and $\left.s_{d}=5 \mathrm{~m}: x_{f A P}=x_{\text {cont }}=145 \mathrm{~m}\right)$. The values of the maximum bending moment increase with decreasing water levels. The highest bending moment out of all positions occurs for grounding at $145 \mathrm{~m}(1.29 \mathrm{E} 7 \mathrm{kNm})$, measured directly at the grounding point.

The shear force at the grounding position is remarkably high compared with the force in the initial floating condition. The effect of running aground can clearly be seen for every position by the large jump of the force value (see figure 3.10-3.12).

The permissible $Q_{T}$ according to $G L$ is exceeded for all stranding points except for the position 232 to $246 \mathrm{~m} \mathrm{AP}$ (see figure 3.9). Groundings at the stern and the bow (stern to $10 \mathrm{~m} \mathrm{AP}, 26$ to $36 \mathrm{~m} \mathrm{AP}$ and $273 \mathrm{~m} \mathrm{AP}$ to bow) lead to critical shear forces for all tide levels. The shear force is outside the envelope for stranding points at stern to $16 \mathrm{~m} \mathrm{AP}, 24$ to $137 \mathrm{~m} \mathrm{AP}, 142$ to $188 \mathrm{~m} \mathrm{AP}$ and $270 \mathrm{~m}$ AP to bow and a surface drawdown higher than $2 \mathrm{~m} .3 \mathrm{~m}$ of tide level results in critical shear forces for the positions stern to 219 and 263 $\mathrm{m}$ AP to bow. And $4 \mathrm{~m}$ water level reduction is critical for accidents that happen at stern to 227 and $253 \mathrm{~m}$ AP to bow. 

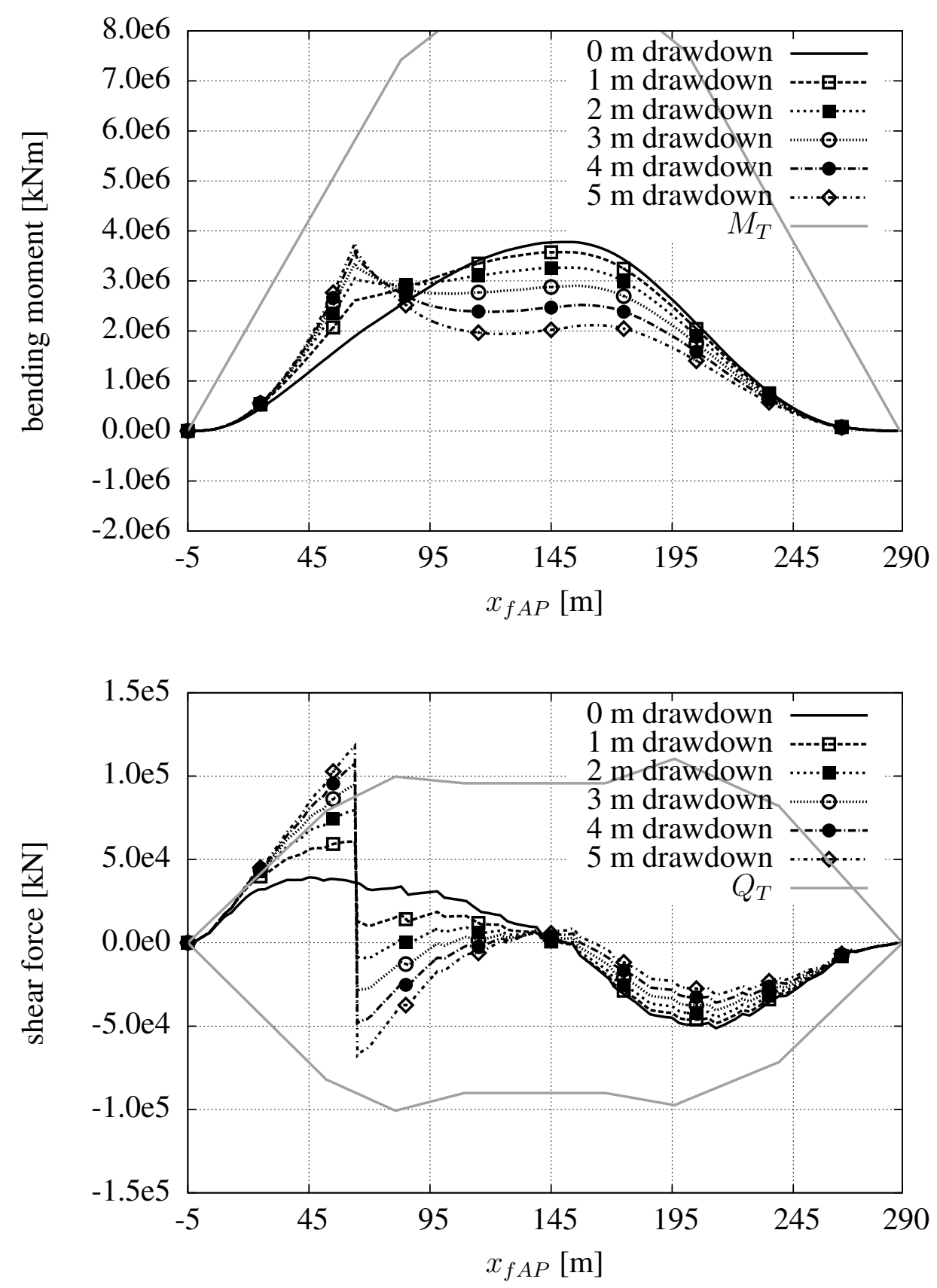

Figure 3.10.: Case A1: bending moments and shear forces for stranding at $65 \mathrm{~m}$ AP. 

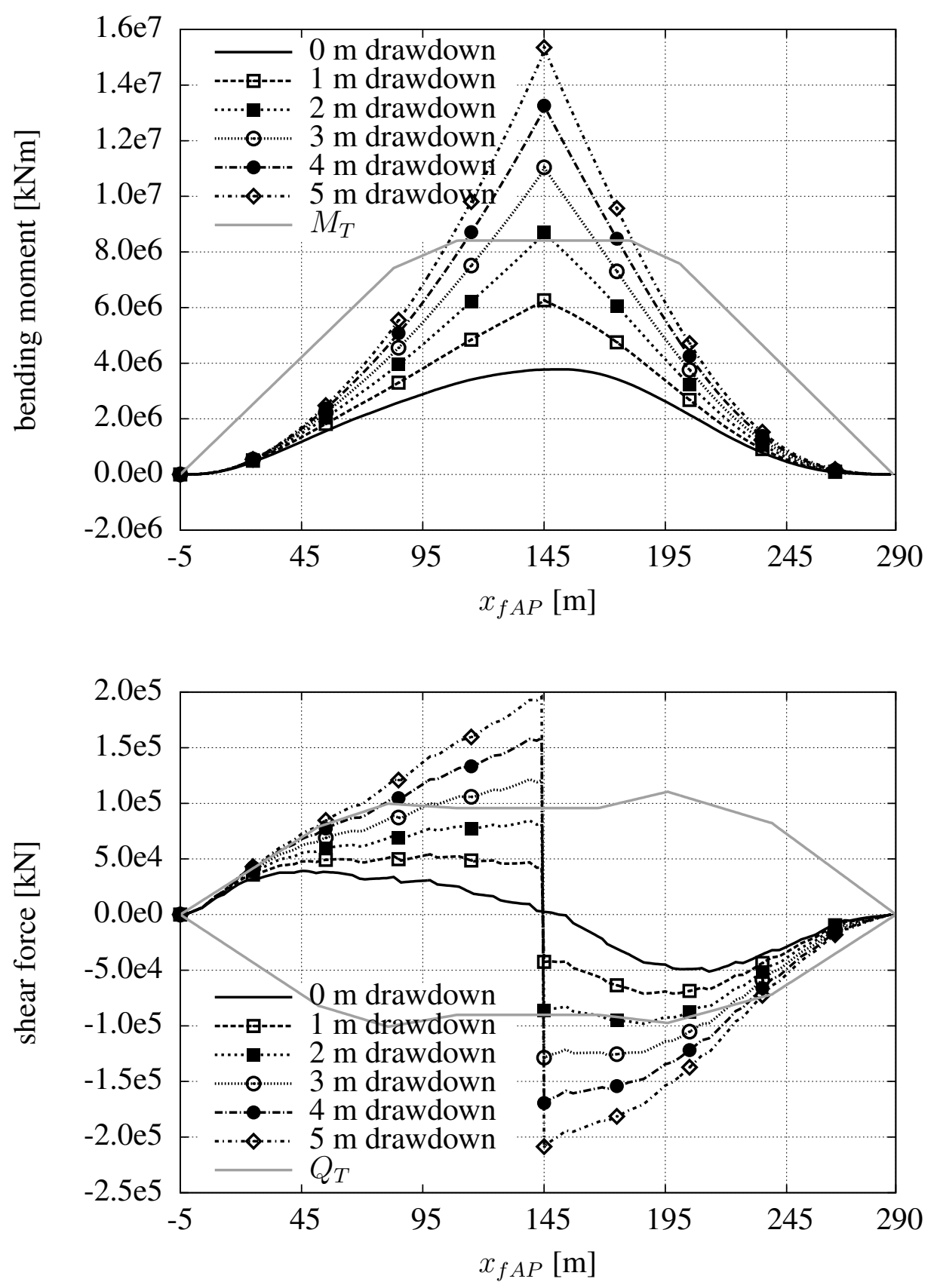

Figure 3.11.: Case A1: bending moments and shear forces for stranding at $145 \mathrm{~m} \mathrm{AP}$. 

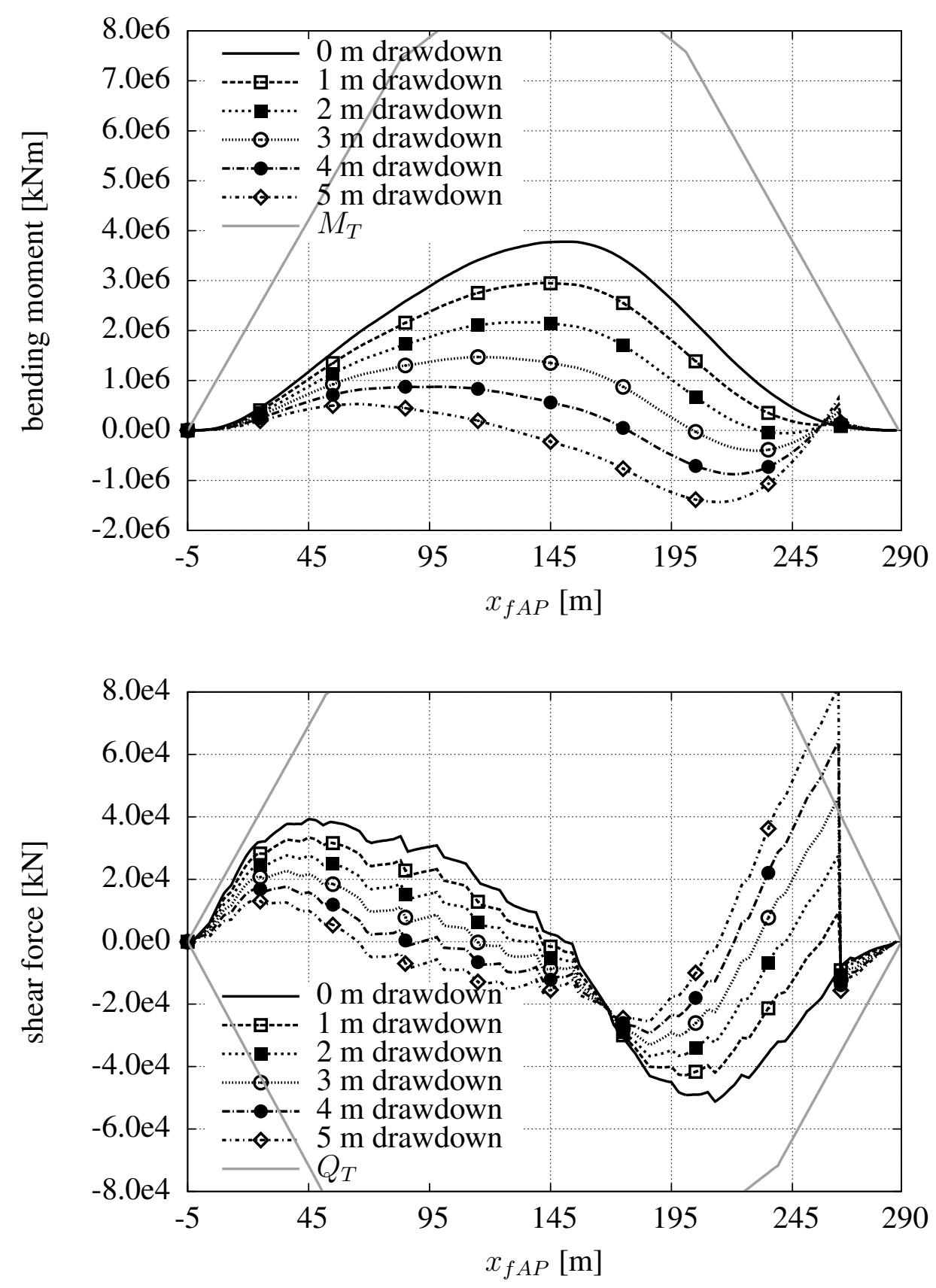

Figure 3.12.: Case A1: bending moments and shear forces for stranding at $265 \mathrm{~m} \mathrm{AP}$. 
The maximum shear force is at the position where the bending moment changes the most. When the vessel strands amidship, the maximum shear force and the maximum bending moment act at the same position. The maximum shear forces for fixed tide levels of $s_{d} 1$ to $2 \mathrm{~m}$ are reached for stranding at the bow $\left(x_{\text {cont }}=279 \mathrm{~m}\right)$. For accidents at $x_{\text {cont }}$ $=125 \mathrm{~m}$ the highest shear forces are measured when the water level is reduced by 3 to 5 $\mathrm{m}$. Thus, stranding at $125 \mathrm{~m}$ AP generates the highest shear force of $1.89 \mathrm{E} 5 \mathrm{kN}$ for $5 \mathrm{~m}$ of surface drawdown out of all calculations. For an accident at $145 \mathrm{~m}$ AP the shear force has a maximum value of $-1.76 \mathrm{E} 5 \mathrm{kN}$. This is five times more than the shear force in the initial floating condition (see figure 3.11) and the contact force is as high as $3.41 \mathrm{E} 5 \mathrm{kN}$.

If one superimposes the critical bending moments and the shear forces with a weighting coefficient $\alpha=0.5$ for a tide level of $5 \mathrm{~m}$ the above described results can be summed up in figure 3.13. In case of $\alpha=0.5$ the exceedance of $M_{T}$ and $Q_{T}$ is equally weighted. The areas in which either the permissible moment or shear force is exceeded are shown in colour. The higher the functional value of $E_{M Q}$ corresponding to the colour scale the more critical is the superimposition.

The plot underlies the results discussed above: The overstepping of $M_{T} / Q_{T}$ is always close to the contact point. The critical cases occur for standing in the midship area and at both ends of the vessel. The critical cases outside amidships are due to the shear forces, which result in the highest value of $E_{M Q}$. The two critical zones in figure 3.13 are also existent in the plots for lower tide levels. The zones expand with increasing $s_{d}$.

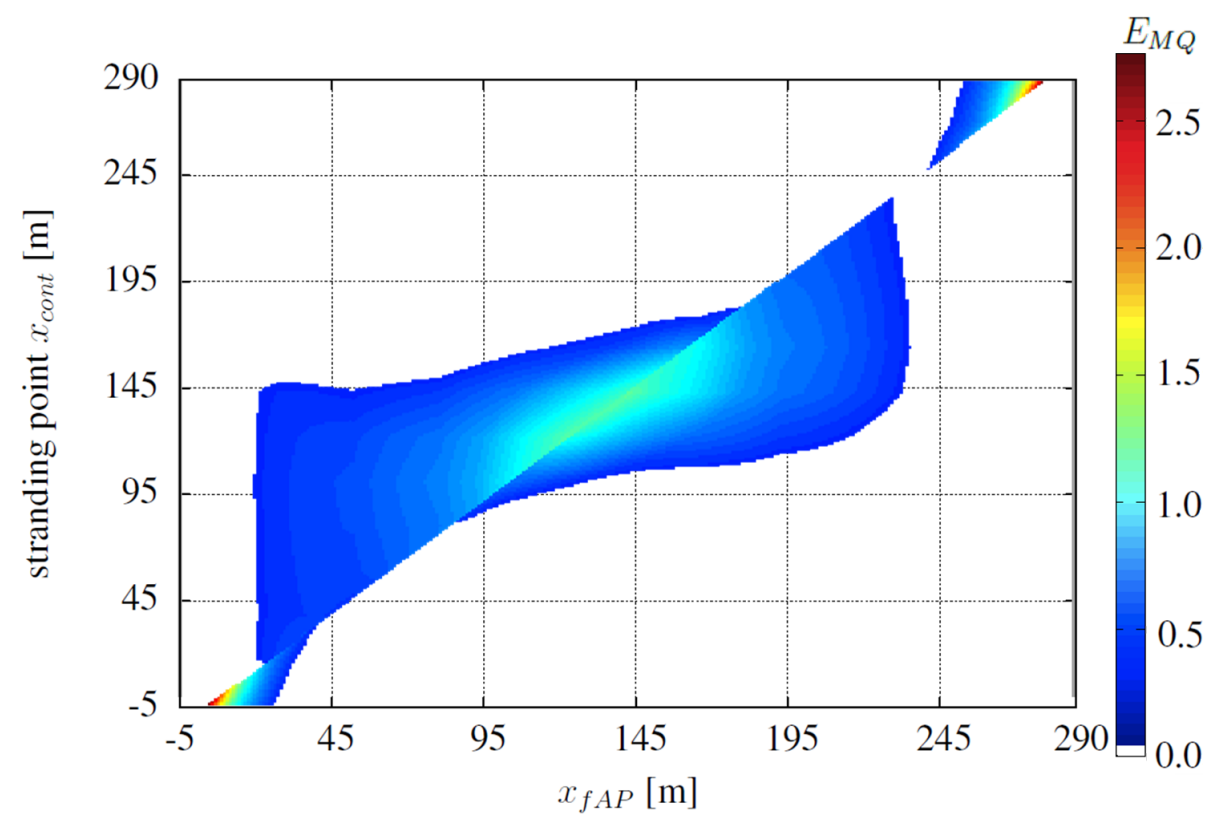

Figure 3.13.: Case A1: superimposition of critical bending moments and shear forces for $5 \mathrm{~m}$ of surface drawdown.

\subsubsection{Stranding case $\mathbf{A 2}$}

Stranding case $\mathbf{A} \mathbf{2}$ is calculated with load case A, without flooded tanks and the reaction force is constantly distributed over $30 \mathrm{~m}$ (10\% Loa). 
Figure 3.14 compares the total bending moments and shear forces for stranding at the area of 130 to $160 \mathrm{~m}$ AP to grounding at the point $145 \mathrm{~m}$ AP for three different tide levels.
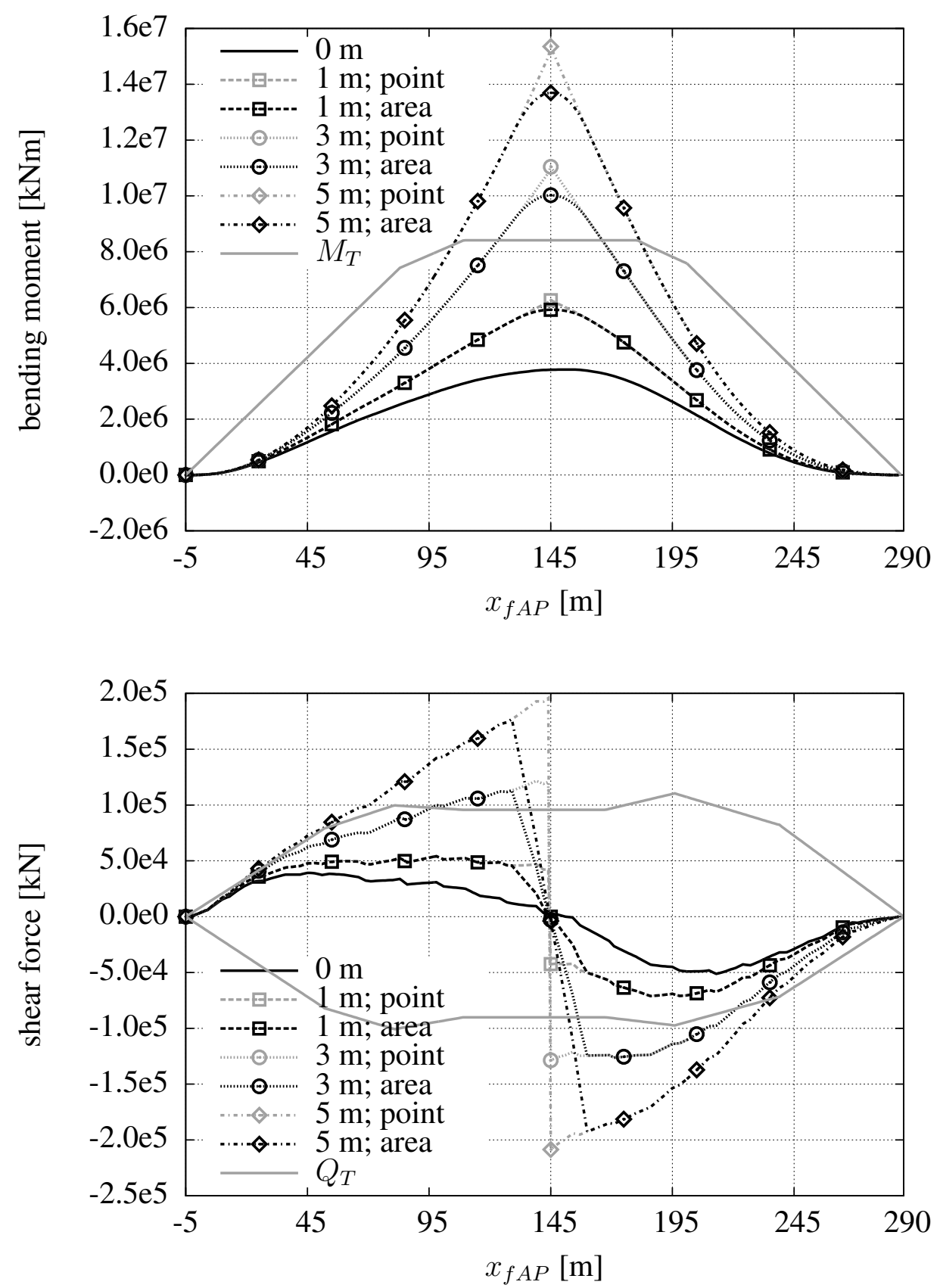

Figure 3.14.: Case A2: bending moments and shear forces for stranding at $145 \mathrm{~m} \mathrm{AP.}$

The bending moments and shear forces are smaller than for grounding at one point around midship. The effect increases with higher tide levels. The average deviation between the moments of case A1 and case A2 measured at $145 \mathrm{~m}$ AP is 8.7\%. The significant shear force change differs by $1.5 \%$. The jump of the shear force now has a gradient different to zero. The force does not jump at the same position, but changes over the 
contact area. A larger but still rigid contact area does not significantly reduce the acting moments or shear forces.

\subsubsection{Stranding case A1y}

Stranding case A1y is calculated with load case A, without flooded tanks and at one stranding points that is located beside the centre line. Stranding outside of the centre line primarily produces a heeling moment. The force due to stranding is small. Table 3.3 gives the stranding force $F_{S}$, the trim and the heel angle for the vessel resting at the point $\mathrm{x}=$ $145 \mathrm{~m} \mathrm{AP}$ and $\mathrm{y}=8 \mathrm{~m}$.

In this case, stability is the problem. The structure is only loaded with low forces and moments compared with case A1. Figure 3.15 reveals that the stranding force $F_{S}$ of case A1 is 10 times higher than in case A1y. Thus, this case will not be considered further.

Table 3.3.: Case A1y: force, heel and trim angle.

\begin{tabular}{rrrrr}
\hline $\mathbf{S}_{\mathbf{d}}$ & $\mathbf{T}_{\mathbf{A P}}[\mathrm{m}]$ & trim $\left[^{\circ}\right]$ & heel $\left[^{\circ}\right]$ & $\mathbf{F}_{\mathbf{S}}[\mathrm{kN}]$ \\
\hline $\mathbf{1}$ & 11.858 & -0.007 & 5.860 & 8547.951 \\
$\mathbf{2}$ & 11.519 & 0.105 & 11.063 & 14721.860 \\
$\mathbf{3}$ & 11.083 & 0.249 & 15.767 & 20439.133 \\
$\mathbf{4}$ & 10.581 & 0.395 & 20.081 & 26543.148 \\
$\mathbf{5}$ & 10.026 & 0.536 & 24.084 & 33413.660 \\
\hline
\end{tabular}

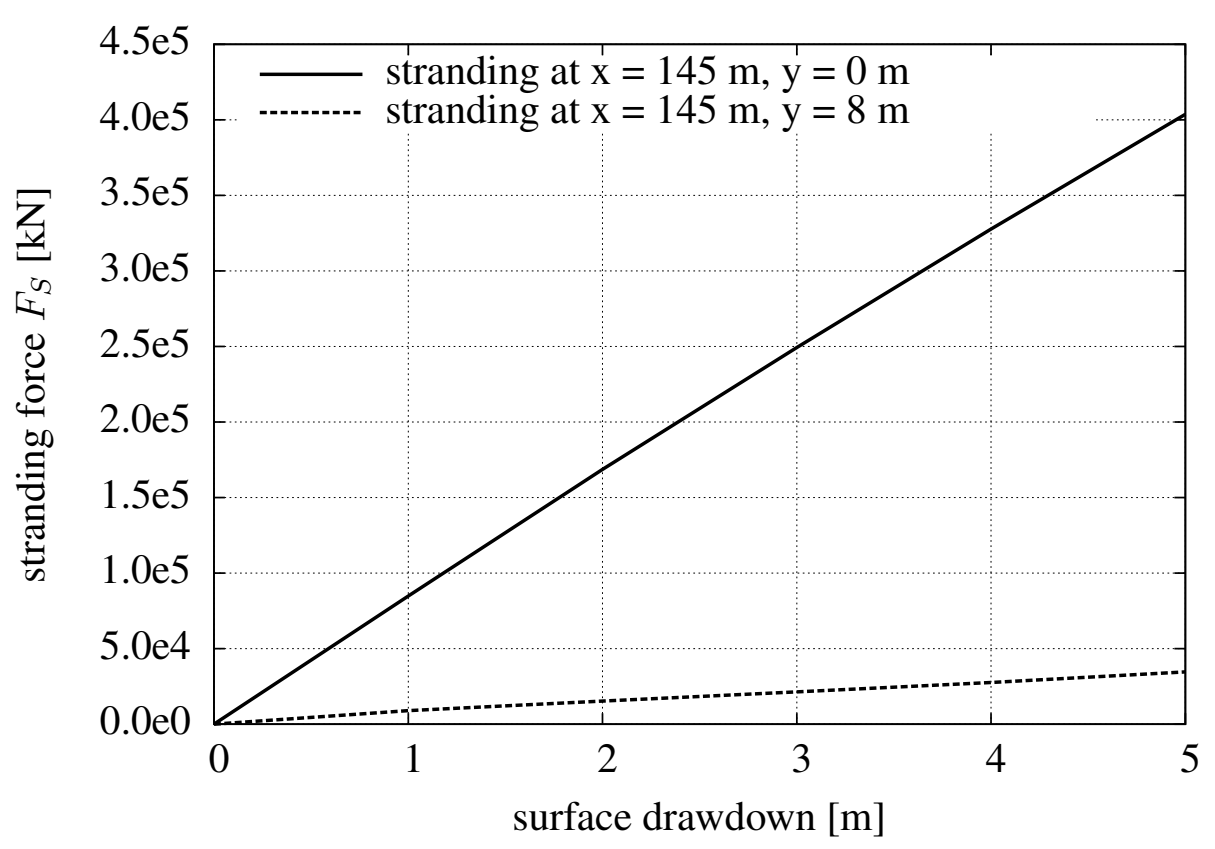

Figure 3.15.: Case A1 vs. A1y: stranding force $F_{S}$. 


\subsubsection{Stranding cases $B 1$ and $C 1$}

Stranding cases B1 and C1 are calculated with load case B and C, without flooded tanks and at three different stranding points $(65,145$ and $265 \mathrm{~m} \mathrm{AP})$ that are located at the centre line. Stranding case B1 and C1 are summed up together. The graphs are shown in appendix A.2.

Load case A is more critical for stranding than any position as load case B. The average deviation between case $\mathbf{A 1}$ and $\mathbf{B} 1$ for the moments and the shear force jump at the stranding point is $41 \%$. Amidships (145 m AP) the five moments are $17.27 \%$ and the shear forces are $18.15 \%$ smaller than in load case A.

Load case $\mathrm{C}$ (departure) is similarly critical to load case A even though the bending moment in the initial floating condition is slightly higher than in load case A. In case C1, the moments are smaller than in case A1. The average deviation of the moments compared to case A1 is $15.58 \%$ when the vessel grounds amidships (see figure A.10). For running aground in the area of aft body, the bending moments are almost the same at the grounding point (e.g. for grounding at $265 \mathrm{~m} \mathrm{AP:} 2.47 \%$ ). The jump of the shear forces is $1.09 \%$ higher for stranding at 65 and $265 \mathrm{~m} \mathrm{AP}$. When stranding amidships the shear forces are almost the same $(0.13 \%$ less than in case A1).

\subsubsection{Stranding case A1f}

Stranding case A1f is calculated with load case A, with flooded tanks and at stranding points that are located at the centre line. Three positions are chosen for the flooding case: 70,145 and $250 \mathrm{~m}$ AP. These positions are located in the middle of empty or partly filled tanks that could be flooded due to a fracture of the outer hull. The effect of flooding is best seen for standing at $145 \mathrm{~m}$ AP because the most water could flood into the tanks compared to the other positions.

There are a half filled ballast tank (x-position: 139.98 to $154.12 \mathrm{~m} \mathrm{AP}$ ) and a $10 \%$ filled HFO tank (x-position: 125.84 to $154.12 \mathrm{~m} \mathrm{AP}$ ) on each side (starboard and portside). It is assumed that the structure fractures shortly after the vessel reached its final stranding position on the ground and the water level reduction begun. So for one meter of surface drawdown the tanks are already flooded and filled with $100 \%$ seawater. If they are flooded a total weight of $1437.54 \mathrm{t}$ is additionally loaded on the structure. Figure A.11 in the appendix A.3 shows the differences in the weight distribution with and without flooding.

In all three calculated cases the influence of the additional weight on the bending moment or shear force distribution is so small that one cannot see a difference in the graphs. That is why in this case no courses of the bending moments and shear forces are shown. The maximum bending moments are $3.96 \%$ smaller than in case A1. Whereas the average jump of the shear forces is $0.55 \%$ bigger. For stranding at $70 \mathrm{~m}$ AP the moments are also smaller $(0.72 \%)$ and the jump of the shear forces is $1.39 \%$ on average higher. If the vessel runs aground at $250 \mathrm{~m} \mathrm{AP}$ and tanks are flooded the effect is reversed. The average deviation of the bending moments to case $\mathbf{A 1}$ is $0.61 \%$ and the shear force jump at the stranding point is $1.26 \%$ smaller.

All tanks located directly at the outer hull are at least partly filled in load case A, so that no significant forces are additionally introduced. The flooding scenario during the already 
extreme conditions of stranding does not have a significant influence on the total bending moment and shear force in this case.

\subsection{Discussion}

In stranding case A1, B1 and C1 (load cases A, B and C, various grounding points at centre line), the bending moments exceed the class permissible $M_{T}$ if the vessel strands in the midship region. Considering the shear force all positions of grounding except 232 to $246 \mathrm{~m} \mathrm{AP}$ are critical. Of all calculations stranding incidents amidship result in the highest shear forces.

In case $\mathbf{A 2}$ (stranding on an area), the moments and forces at the grounding position are reduced compared with case A1. The results of case $\mathbf{A 1}$ are conservative. In reality, the ship lies on an area when running on soft ground.

Stability is the problem when the grounding point/area lies outside the centre line. The forces and moments due to grounding are small. This case does not need to be further examined for the structural analysis.

The three calculated load cases and the flooding case reveal that different loading conditions do not have much influence on the total moments and shear forces. Stranding already produces very high changes in the bending moments and shear forces.

The shear force due to grounding seems to be critical. Especially in combination with the maximum bending moment at the same position, the structure can sustain severe damage. The bending moment around the grounding position can be detrimental. This effect is not significantly reduced when the vessel runs aground on a sandbank.

\subsection{Conclusion}

A method is introduced for calculating the total bending moment, the shear force and the heel angle for a ship during soft grounding. Flooding of tanks and different load cases can be taken into account. An optimisation tool is implemented for an easy and accurate representation of the results. It is possible to superimpose the critical cases and even weight the influence of bending moment versus shear force.

If the ship lies in the final position on the ground and the water level is reduced, the resulting bending moments and shear forces depend on the external forces. The distributions of the weight and of the hydrostatic forces are relevant. The weight does not change unless flooding is included. The hydrostatic forces on the hull depend on the water level and the trim, which result from the new equilibrium position. In the proposed method, the new equilibrium position is determined on the basis of the real ship form. The advantage of the proposed method is that the correct hydrostatic forces, as a function of water level and stranding position, are used to determine the bending moments and shear forces due to stranding. The method is not limited to a specific ship type. Any vessel with any load case can be used.

The critical combinations of grounding point/area, surface drawdown and load case (with or without flooding) that lead to an exceedance of the class permissible $M_{T}$ and $Q_{T}$ can 
be calculated. With the help of the optimisation tool all critical combinations can be determined at once even for parameters that lie in between calculated cases. The comparison with existing formulas showed that the presented method gives reasonable results. The method is quick and applicable for every existing vessel and to every ship design to estimate the acting moments and forces.

Once the acting forces and moments as well as the sections at risk are known, the next step is to reduce the simplification. The method cannot be used to give information about local and global damage, the influence of the ground and the point of total collapse of the structure. The method is further used to control the global forces, moments and reactions in the simulation of stranding scenarios.

Load case A is chosen for further calculations. Stranding case A1 serves as a comparison. 


\section{Simulation of stranding scenarios}

This chapter ties in with the previous chapter and the underlying stranding scenario is the same as already defined in subsection 2.1. The acting forces and moments as well as the sections at risk are known from chapter 3 . But this method is not capable of giving information about the structural resistance to the accidental load, the local and global damage of the structure, the interaction of bending moment and shear force and the influence of the ground. Thus in this chapter a simulation method based on the FE method is presented to gain all relevant details of stranding. The method can be used to calculate real incidents.

The sections at risk are modelled according to the construction drawings and the ground is represented by a geotechnical material model. To accurately simulate limit load capacity the loads rather than a curvature are applied. The load increase has to be physically realistic and it is proven that the ship remains stable and does not capsize. This is the reason why the method introduced in section 3 is used to control the global forces, moments and reactions in the FE simulation of the stranding scenarios.

Three stranding positions are chosen for simulation: 65,147 and $250 \mathrm{~m}$ AP. Thus in each part of the ship stranding is exemplarily simulated. The part of the vessel that experiences the highest moments and shear forces is amidships. The fore body and aft body parts are additionally analysed because here the shear forces are detrimental. At each stranding location the geometry of the ground is varied. In addition all scenarios are also simulated with a rigid ground to have a conservative comparison case.

A special focus is placed on the ultimate hull girder strength and the influence of the combination of high external bending moments and shear forces.

First the simulation method is described and verified and followed by the specification of different parameters that are discussed with regard to optimal results and short calculation time. One scenario is additionally compared to a simpler model. A double T-beam is chosen to reveal the stress distribution over the model's height. Then the results of the midship scenarios are given in detail. The results of the method are further compared to the Fowairet stranding incident. At the end of the chapter the results for bow and stern scenarios are discussed briefly.

\subsection{Description of simulation method}

Although the problem is quasi-static, it is solved with $L S$-DYNA [38] explicitly because of the complex contact problem (see section 2.5). Comparative calculations with an implicit 
FE program (ANSYS) revealed that the model is extremely instable due to the contact and the highly different material properties of sand and steel.

The FE models consists of three different parts. A detailed shell-model of three cargo holds at the stranding point is generated. The rest of the ship is represented by beamelements. The ground is reproduced with shell or volume elements depending on the parameters. Half of the vessel and the ground are modelled and symmetry conditions are used. The structural arrangement in the midship and bow section are identical on starboard and port side. At the aft body the bearing structure is the same on both sides, only the spaces above the machinery room differ. Thus the important structural arrangement are reproduced. It is assumed that the collapse is symmetrical and that the direction of the crack is irrelevant. Using symmetry is standard within ultimate load calculation and classification to save computational effort (see among others Germanischer Lloyd [18]).

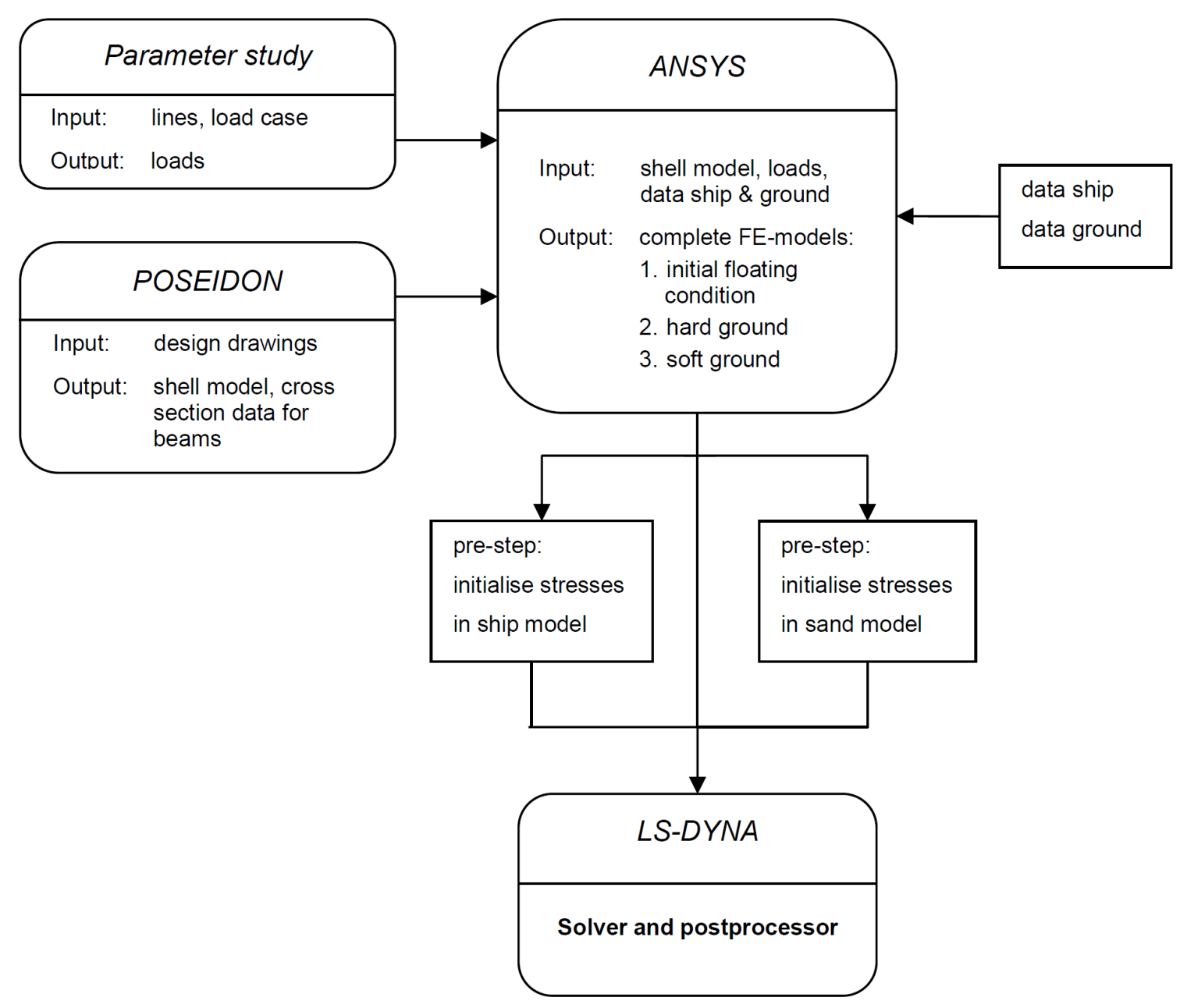

Figure 4.1.: Flow chart of the simulation method.

Figure 4.1 shows the procedure of the simulation method. The shell model is generated with POSEIDON [17] and exported to ANSYS. All other information (cross section data for the beam elements, ship and ground geometries, material data etc.) are also passed to ANSYS, which is used as preprocessor. The light ship and dead weight as well as the equivalent buoyancy depending on the tide level are calculated as in the parameter study 
(see chapter 3) and transferred to ANSYS as load curves. Three models are the output. The first model represents the vessel and exists of beam and shell elements. It is loaded with the forces according to the initial floating condition to initialise stresses and strains via a pre-step in Implicit $L S$-DYNA. The second model only includes the hard ground. The third model represents the soft ground, which is also transferred to Implicit LS-DYNA to initialise stresses and strains due to its dead weight. The results from the pre-step of the first model are combined with the hard or soft ground and the load curves depending on the tide levels. The problem is finally solved in Explicit LS-DYNA.

\subsubsection{Ship model}

Three detailed parts of the vessel Postpanmax (see table 2.4) are modelled with shell elements to consider three different stranding points. The extensions are listed in table 4.1. The centre of the stranding area lies in the middle of the shell section to exclude boundary effects. Only in the stern part the stranding point is not exactly in the middle as a result of the aft body construction. The models include all relevant structural elements given in the construction drawings. A drawing of a midship frame is shown in figure 4.3. For the drawings of the stern and bow section please refer to the appendix B.2.

The generation of the ship model is exemplarily described for the midship section and applies to the other sections. Figure 4.2 presents the entire midship model with one possible sandbank geometry.

Table 4.1.: Shell models for stranding simulation.

\begin{tabular}{lrrr}
\hline & stranding point & extension & shell elements \\
\hline stern & $65 \mathrm{~m} \mathrm{AP}$ & 37 to $82 \mathrm{~m} \mathrm{AP}$ & 168000 \\
midship & $147 \mathrm{~m} \mathrm{AP}$ & 126 to $168 \mathrm{~m} \mathrm{AP}$ & 163000 \\
bow & $250 \mathrm{~m} \mathrm{AP}$ & 228 to $270 \mathrm{~m} \mathrm{AP}$ & 153000 \\
\hline
\end{tabular}

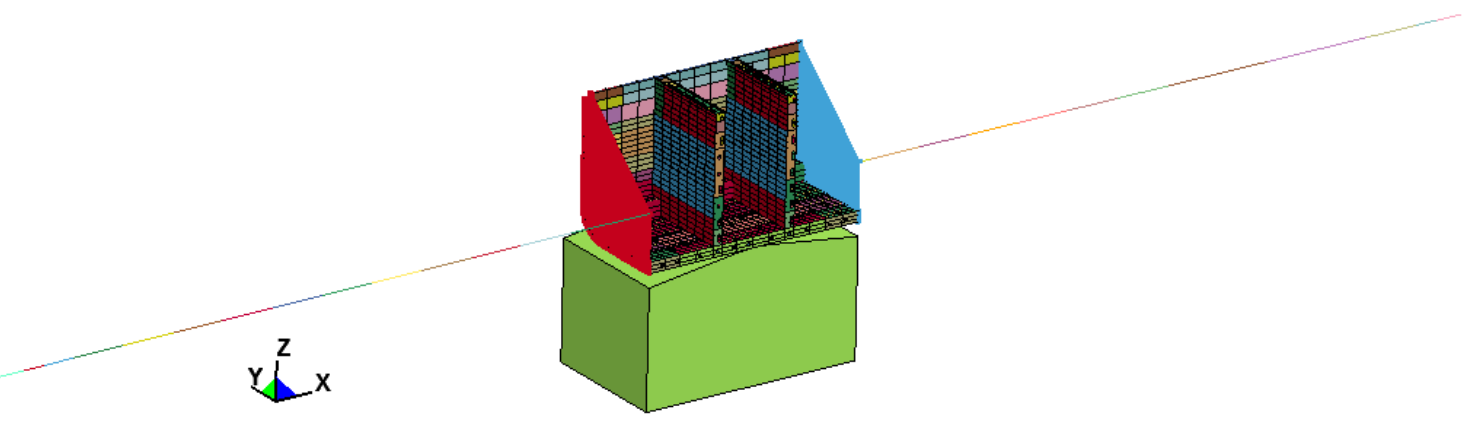

Figure 4.2.: Midship model with sandbank.

The detailed part of the vessel is modelled with Belytschko-Tsay elements (type 2) (see LS-DYNA manual [38]). The element type is simple integrated, very fast and each node has eight degrees of freedom. The mesh is regular and has a size of 200-250 mm 
and the number of shell elements is given in table 4.1.

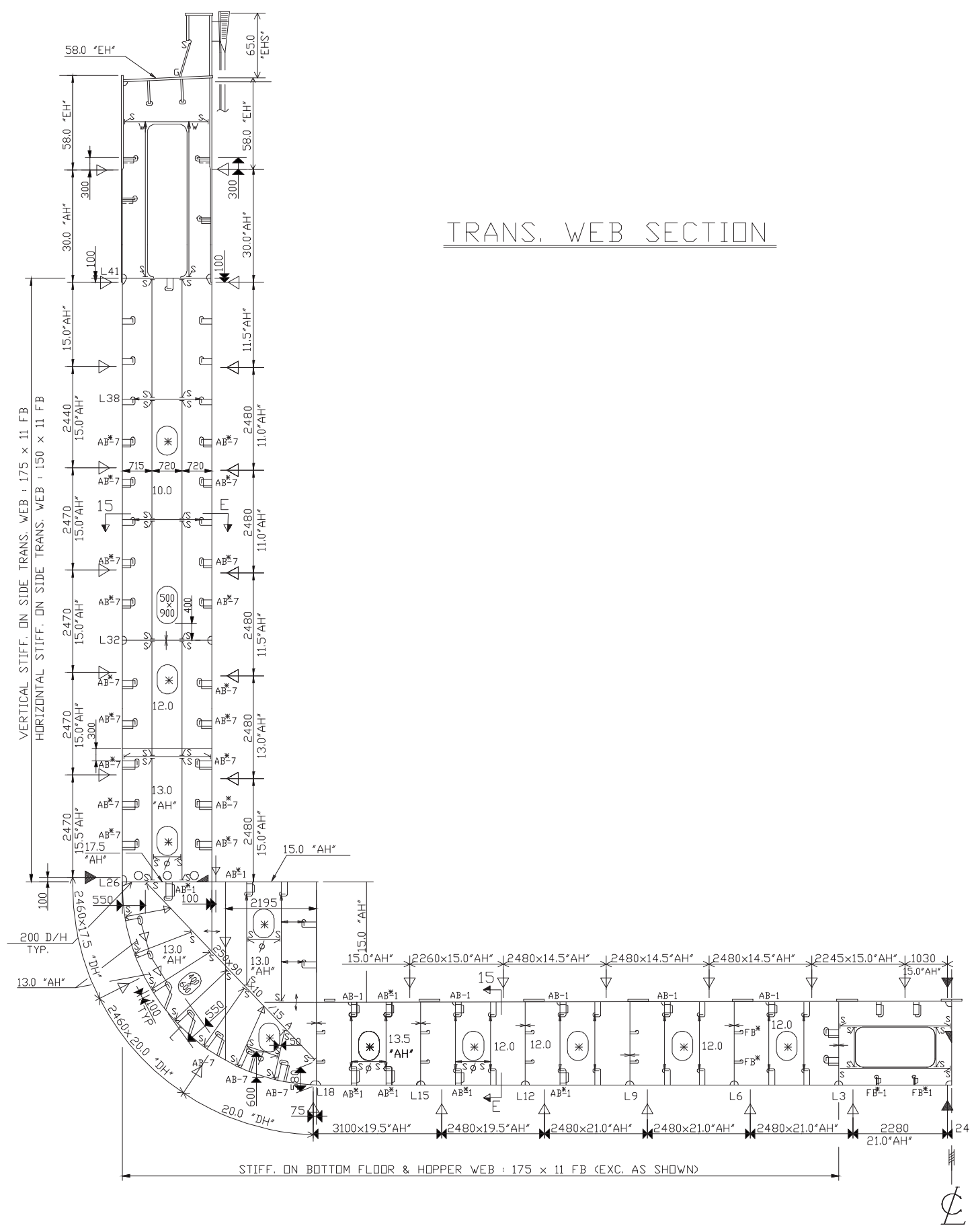

Figure 4.3.: Construction drawing of midship frame.

The material model of the shell elements is nonlinear with regard to strain hardening. True stress-strain curves and the coefficient of friction between steel and steel are known from experiments performed by the Institute of Ship Structural Design and Analysis located at Hamburg University of Technology. The curves are scaled for the different yield 
stresses and taken into account via the modified piecewise linear plasticity material model (type 123) (see figure 4.4). The yield stresses according to the construction drawings are 235 (A), 315 (A32) and $355 \mathrm{~N} / \mathrm{mm}^{2}$ (A36). The coefficient of friction for all steel materials is 0.23 , also known from Karlsson et al. [29].

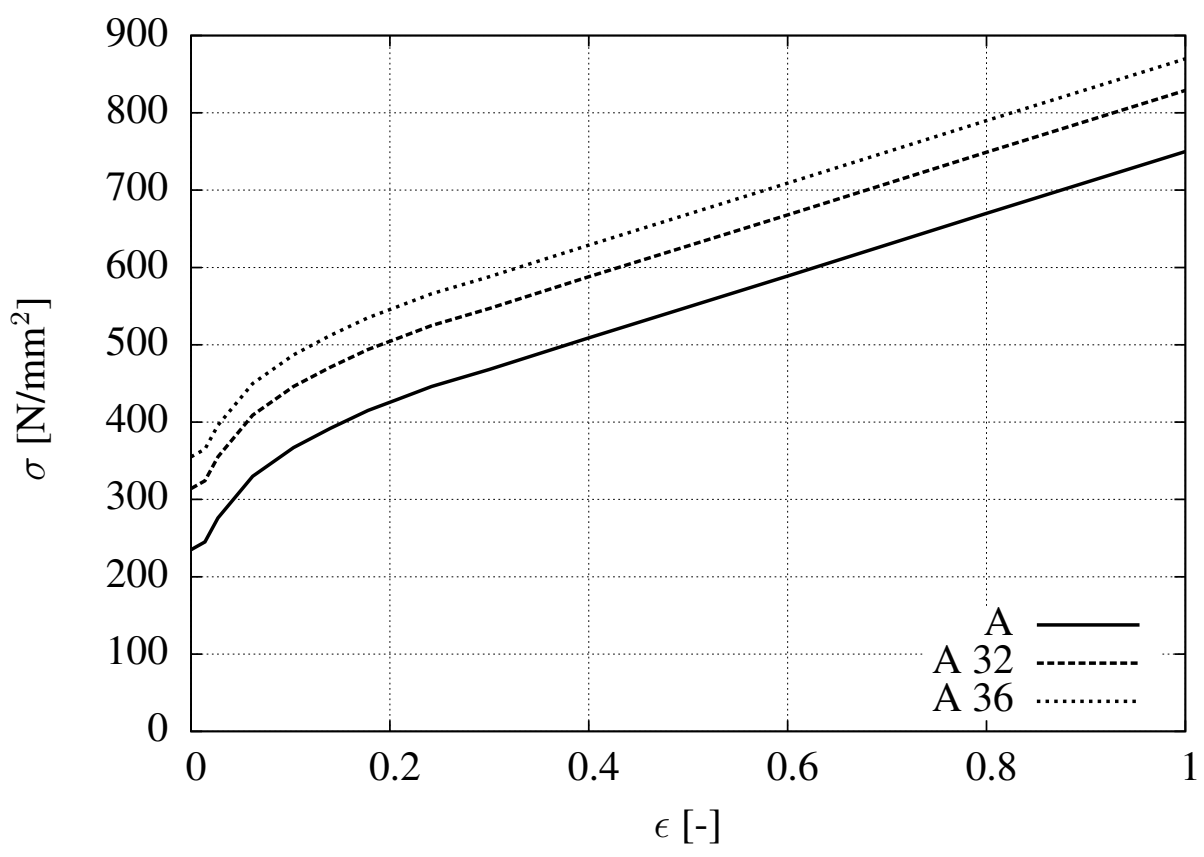

Figure 4.4.: True stress-stain curves.

The universal failure criterion developed by Peschmann [54] is implemented as given in equation (4.1). The criterion was developed by fitting experimental results with the FE program $L S$-DYNA for numerical simulations.

$$
\begin{array}{ll}
\epsilon_{k}=0.1+0.8 \cdot \frac{t}{\ell} \quad \text { for } t<12 \mathrm{~mm} \\
\epsilon_{k}=0.08+0.65 \cdot \frac{t}{\ell} \quad \text { for } t>12 \mathrm{~mm}
\end{array}
$$

The critical strain $\epsilon_{k}$ depends on element thickness $t$ and element edge length $\ell$. When the strain of an element reaches the value $\epsilon_{k}$ the element is deleted and loads, moments or stresses are no longer carried. Since the calculation is quasi-static, the strain rate has no influence and can be disregarded.

One half of the edge cargo holds has no failure criterion. Otherwise the introduction of the global forces and moments cannot be guaranteed. These edges of the shell model will not be used for any analysis to exclude boundary effects.

The beam elements represent the rest of the ship (fore and aft body) and level with the neutral axis. The beams are connected with constrained equations at both ends of the shell-model. The elements (Belytschko-Schwer resultant beam) are $2 \mathrm{~m}$ long and their cross-sectional data equal those of the real ship. In the appendix, figure B.1 shows the 
implemented and original moment of inertia $I_{y}$. The real moment of inertia has peaks according to the stiff cross sections as bulkheads. The peaks are smoothed as usual for finite-element calculations. The constrained equations guarantee that the cross sections remain planar. The material model of the beam elements is ideal elastic. All beam nodes are restricted in y-direction because of the symmetry condition.

Line and pressure loads are applied to the beam and shell elements according to the stranding scenario. The forces equivalent to the sum of loading, weight and buoyancy forces are generated with the method presented in section 3 whereas the buoyancy at the initial contact area (between hull and sand) is deleted. The calculated forces are distributed over the entire inner double bottom within the shell model. All forces are always normal to the element axis. The cargo load application over the entire inner double bottom is conservative for a container vessel, because in reality loading forces are only exerted on the bottom structure at the bulkheads. There are no forces from the cargo on the inner double bottom which counteract the contact forces.

The initial floating condition is solved via a preliminary calculation. Thus the model has initial stresses and strains as well as displacement due to the initial floating condition at the start of the explicit calculation. The loads are applied in the time domain during the explicit solution. The load curves start with the initial floating condition and end with the loads equal to a maximum of $5 \mathrm{~m}$ of water level reduction. Each meter of surface drawdown is applied over $10 \mathrm{~s}$.

A single-surface contact including all elements is chosen to prevent nodes penetrating element surfaces. Tests revealed that the contact type is not more expensive than a locally applied contact algorithm.

\subsubsection{Ground models}

Two different ground models are used. The Mohr-Coulomb material model is considered for the soft ground calculation. All simulations with the soft ground are named with ' $\mathbf{S}$ ' as the first character. For comparison the ground is also calculated with rigid material (first character of the titles is ' $H^{\prime}$ ). The geometry and the mesh of the ground surface is in both cases the same.

\section{General description of the ground}

Four sea floor geometries are listed in table 4.2. The slope angle $a$, the length $p$ and the width $w$ of the plateau are varied and are shown in figure 4.5 (case Sa7p2). The geometries are considered to be representative for typical ground conditions in discussion with members of Institute of Geotechnical Engineering and Construction Management located at Hamburg University of Technology and with members of Emergency Response Service of GL. 


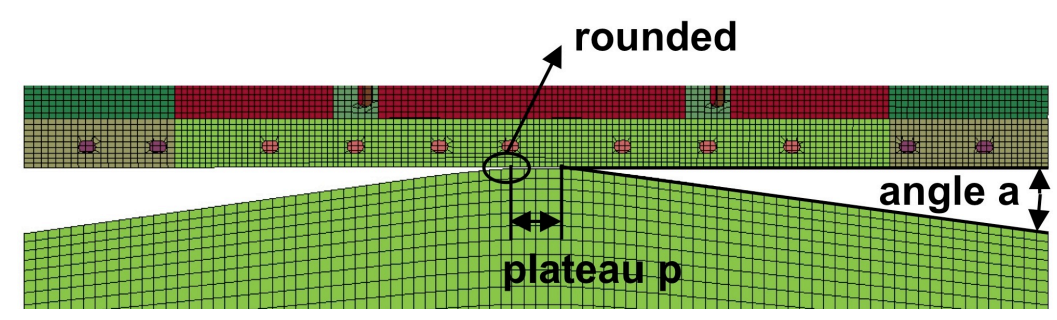

Figure 4.5.: Geometry of ground (Sa7p2).

Table 4.2.: Geometry of ground.

\begin{tabular}{lrrr}
\hline case & angle a & plateau p & width w \\
\hline a7p2 & $7^{\circ}$ & $2 \mathrm{~m}$ & $\infty$ \\
a7p22 & $7^{\circ}$ & $22 \mathrm{~m}$ & $\infty$ \\
a7p22w10 & $7^{\circ}$ & $22 \mathrm{~m}$ & $10 \mathrm{~m}$ \\
a15p2 & $15^{\circ}$ & $2 \mathrm{~m}$ & $\infty$ \\
\hline
\end{tabular}

The modelled ground always has the same length as the shell model. The sharp edges at the plateau are rounded to exclude structural damage due to a sharp edge. The middle of the plateau levels with the stranding points 65,147 or $250 \mathrm{~m}$ AP.

The above described single-surface contact is sufficient to guarantee no penetration of ship and ground. If elements of the outer shell plating are deleted elements of the double bottom still have a contact condition with the ground.

\section{Hard ground}

The hard ground model represents a bluff rock. It is a conservative but rare stranding scenario (see e.g. Wang et al. [81]) and represents the upper bound of ground stiffness. The hard ground calculations are also used to identify the influence of different ground stiffness on the collapse of the hull girder.

The ground is represented by four-node Belytschko-Tsay elements and material type rigid (type 20). Young's modulus is the same as for the elements of the outer shell plating, which is only needed to calculate the contact stiffness. Boundary conditions are defined via the material definition. The ground is fixed in all directions.

\section{Soft ground}

The material properties of soil are needed to adequately represent the so-called soft ground. Compactness and patterns in the arrangement of the particles as well as the pore size and pore fluid distributions define the capacity of the soil.

A typical soil type in rivers, at coasts or in harbours is sand where the pores are filled with water. Thus the soft ground consists of saturated sand. Sand is typically non-cohesive ( $c$ $=0)$ i.e. the physical properties of the grains are determinant. The unit weight $\gamma_{r}$ gives 
the ratio of mass and volume. For saturated sand the buoyant unit weight $\gamma^{\prime}$ is relevant. It includes the unit weight of water $\gamma_{w}$ :

$$
\gamma^{\prime}=\gamma_{r}-\gamma_{w}
$$

There are different models to describe the deformation and strength behaviour (see e.g. Grabe [20], Gudehus [22] and Kolymbas [30]). Most relationships used to characterise the stress-deformation and strength properties are empirical and based on phenomenological description of soil behaviour. The Coulomb equation (4.3) is by far the most widely used for strength. Equation (4.3) and figure 4.6 clarify that the shear strength $\tau_{f}$ is dependent on the cohesion $c$, the stress $\sigma$ and the angle of friction $\varphi$. A ground with a stress state that lies above the failure surface collapses.

$$
\begin{aligned}
& \tau_{f}=c+\sigma \cdot \tan (\varphi) \\
& \tau_{f}=c^{\prime}+\sigma^{\prime} \cdot \tan \left(\varphi^{\prime}\right)
\end{aligned}
$$

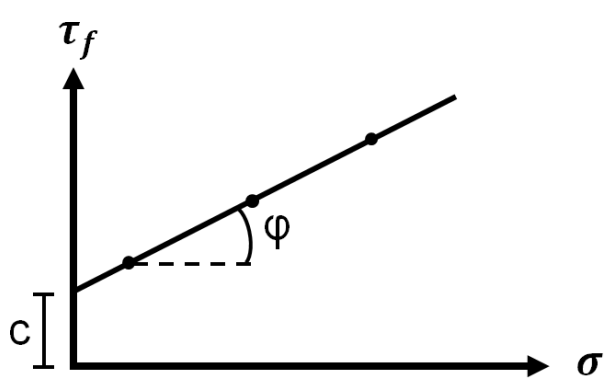

Figure 4.6.: Shear strength $\tau_{f}$ as function of normal stress $\sigma$ (Grabe [20]).

In saturated sand only effective stresses $\sigma^{\prime}$ are active because water cannot carry shear stresses. Thus equation (4.4) is relevant with the effective cohesion $c^{\prime}$ and the effective angle of friction $\varphi^{\prime}$. The effective stress $\sigma^{\prime}$ adds up to:

$$
\sigma^{\prime}=\sigma-u \text { with } \sigma^{\prime}=\gamma^{\prime} \cdot z
$$

Parameter $z$ is the depth below ground surface and $u$ is the pore-water pressure. It is disregarded that depending on the permeability excess pore-water pressure may occur during stranding.

The stiffness of the soil is given by the stiffness modulus, whereas the void ratio $e$ can be calculated by equation (4.7):

$$
E_{s}=\sigma^{\prime} \cdot \frac{1+e}{C_{c}}
$$




$$
e=e_{0}-C_{c} \cdot \ln \frac{\sigma^{\prime}}{\sigma_{0}}
$$

$C_{c}$ is the compression index and $e_{0}$ is the initial void ratio. Both values are given in table 4.3 for the reference stress of $\sigma_{0}=10 \mathrm{kN} / \mathrm{m}^{2}$ of compact fine sand (see Gudehus [22]).

Soil can increase its volume by reducing the compactness. This happens if shear forces are acting and until the shear strength is reached. This property is described by the (effective) angle of dilatancy.

The Mohr-Coulomb model includes no post-failure modelling and no strain rate effects. It is perfectly elsato-plastic. However, the limited number and simple interpretation of input parameters make this model a good choice for the purpose of this work. The model enables soil to be modelled using familiar geotechnical parameters. It captures soil plasticity behaviour very well and ensures a unique solution.

In $L S$-DYNA material type 173 is based on equation (4.3). The material has a MohrCoulomb linear shear failure surface, where $\tau_{f}$ is the maximum shear stress on any plane, $\sigma$ is the normal stress on that plane. The tensile strength is not relevant for non-cohesive soil and not used within this work. After the material reaches its tensile strength, further tensile straining leads to volumetric voiding. Volumetric response to hydrostatic pressure is linear and defined by the elastic shear modulus and Poisson's ratio values provided by the user. All input parameters are listed in table 4.3. The buoyant unit weight $\gamma^{\prime}$, effective angle of friction $\varphi^{\prime}$, effective angle of dilatancy $\Psi^{\prime}$, effective cohesion $c^{\prime}$ and Poisson's ratio $\nu$ are given for compact fine sand.

Table 4.3.: Material data of saturated sand.

\begin{tabular}{lr}
\hline buoyant unit weight $\gamma^{\prime}\left[\mathrm{kN} / \mathrm{m}^{3}\right]$ & 11.00 \\
effective angle of friction $\varphi^{\prime}\left[{ }^{\circ}\right]$ & 35.00 \\
effective angle of dilatancy $\Psi^{\prime}\left[{ }^{\circ}\right]$ & 5.00 \\
cohesion $c^{\prime}[\mathrm{kPa}]$ & 0.00 \\
Poisson's ratio $\nu[-]$ & 0.30 \\
initial void ratio $e_{0}$ & 0.50 \\
compression index $C_{c}$ & 0.005 \\
\hline
\end{tabular}

The material model is only valid for Lagrangian solid elements. The chosen element type is a constant stress solid element (type 1) (see LS-DYNA manual [38]). The elements are eight nodes cuboid and the nodes have six degrees of freedom each. The element type has a trilinear displacement approach and is single-point integrated.

The mesh is only finer at the top of the sandbank to represent the imprint of the vessel and to save calculation time. Different mesh sizes with different numbers of elements are tested. The calculation time depends on the element numbers whereas the results of the vessel do not depend on the mesh size of the ground.

The bottom elements are restricted in z-direction. The side elements have either the symmetry condition or are allowed to move in z-direction. The total height of the sandbank is 
determined by weighting up the height versus the calculation time. An adequate height of the sandbank is reached if no influence of the loading is measured at the bottom elements. For the finally chosen height of $25 \mathrm{~m}$ the vectors representing the first main stress axis are horizontal in the bottom elements. The calculation time is not increased compared to a height of $15 \mathrm{~m}$ if the element number is the same. The mesh size at the top is the same for all tested heights.

The stiffness of the soil is calculated with equation (4.6). In LS-DYNA a gradient of the shear modulus versus the height of the sandbank can be provided by the user for the depth dependency.

Initial stresses in the sandbank are needed for an accurate calculation (see equation (4.5)). To initialise stresses the sand is loaded with gravity in a quick pre-calculation step. The implicit solver is used for the pre-step.

The friction between sand and steel is determined by equation (4.8) with the angle of wall friction $\delta$ given in Grabe [20]. There is no relative movement between sandbank and vessel, so a dynamic coefficient of friction is not relevant.

$$
\mu=\tan (\delta) \text { with } \delta=\frac{2}{3} \cdot \varphi
$$

\subsection{Verification of the simulation method}

\subsubsection{Ship model}

The model with the rigid ground is calculated with an ideal elastic material and without the failure criterion (Ha7p2el).

Figure 4.7 shows in black the total bending moment of the cross section at $147 \mathrm{~m} \mathrm{AP}$ for Ha7p2el. The acting moment and shear force are achieved by equations 4.9 and 4.10 .

$$
\begin{gathered}
M_{h}=\int_{A} \sigma_{x} \cdot z d A \\
Q_{h}=\int_{A} \tau_{z} d A
\end{gathered}
$$

The bending moment $M_{h}$ and shear force $Q_{h}$ are plotted over the surface drawdown (see figure 4.7 and 4.8). The corresponding moments of case $\mathbf{A 1}$ from chapter 3 are given in grey. The moment of Ha7p2el is almost equal to that of case A1. In simulation Ha7p2el the vessel lies on a small area, whereas the beam has one contact point. The differences are comparable to those between case $\mathbf{A 1}$ and $\mathbf{A 2}$ (see subsection 3.3.2). With receding tide the differences increase because the initial contact length of $2 \mathrm{~m}$ rises. It is doubled for a surface drawdown of $5 \mathrm{~m}$. If the beam is also calculated with, e.g. a contact area of $4 \mathrm{~m}$, the moments and forces are identical for $5 \mathrm{~m}$ of receding tide. Case $\mathbf{A 1}$ is chosen as a reference in all graphs because the contact area is not known before the stranding scenarios are solved. 


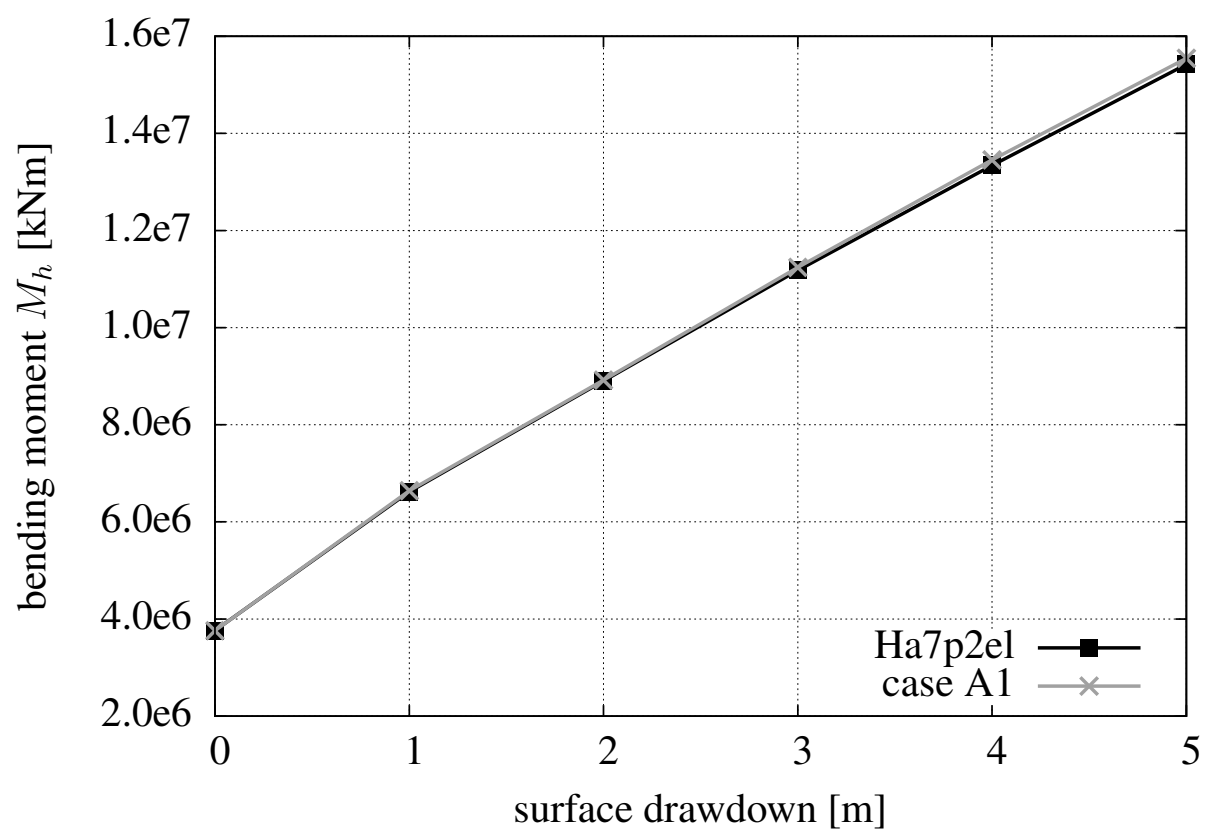

Figure 4.7.: Ha7p2el: bending moment $M_{h}$ at $147 \mathrm{~m} \mathrm{AP}$.

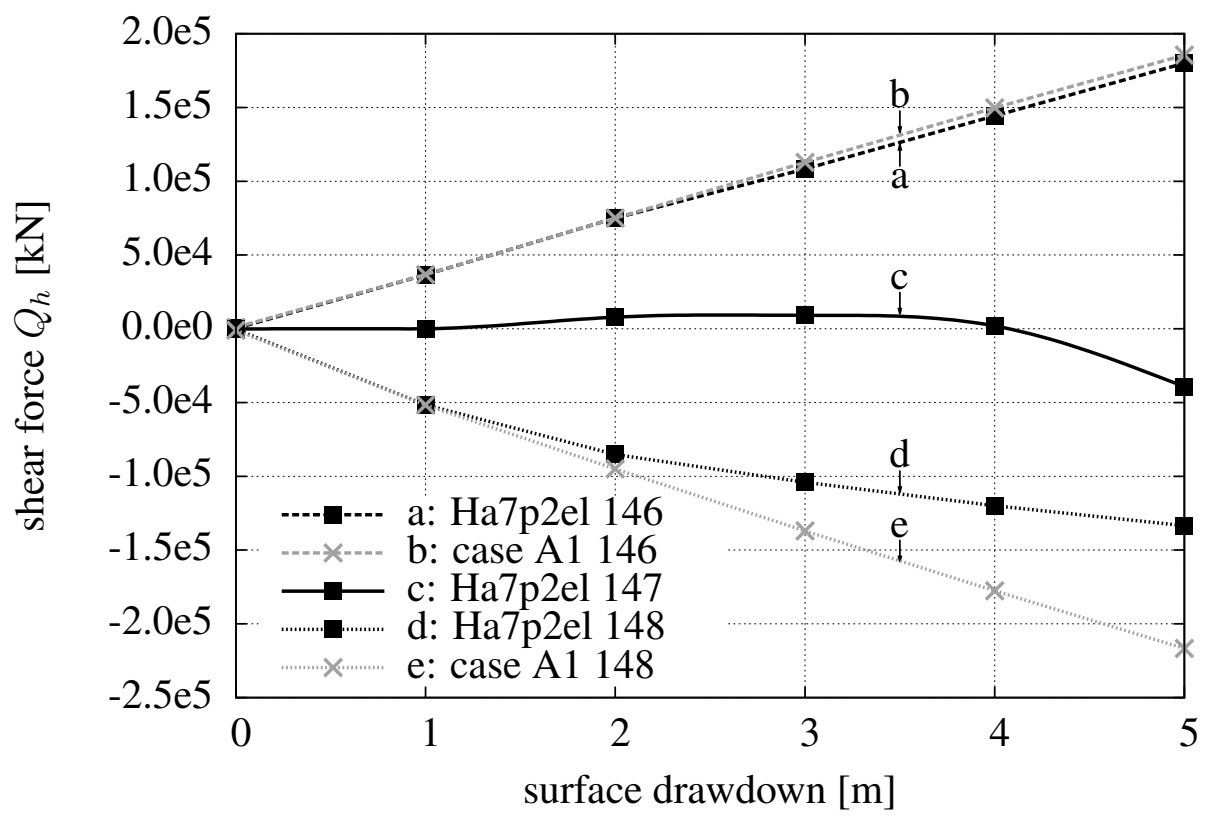

Figure 4.8.: Ha7p2el: shear forces $Q_{h}$ at 146, 147 and $148 \mathrm{~m} \mathrm{AP.}$

The vessel can bear the same forces and moments that are globally applied. The simple beam theory is valid for both cases, because no nonlinear effects are existent.

The corresponding shear forces can be found in figure 4.8. The shear force at $147 \mathrm{~m} \mathrm{AP}$ of case A1 cannot be given because of the shear force jump. Thus the total shear forces at 146 and $148 \mathrm{~m} \mathrm{AP}$ are additionally given for both methods. The forces accentuate the shear force jump around the contact point/area (see also fig. 3.11). The discrepancy be- 
tween case A1 and Ha7p2el reveals the effect of the contact area. The vessel trims to the bow and the contact area is increased in bow direction. This effect cannot be reproduced by the method presented in chapter 3. Thus the forces at $148 \mathrm{~m}$ AP differ to a greater extent than those at $146 \mathrm{~m}$ AP. The change of sign in curve $147 \mathrm{~m}$ AP is the result of the increasing contact area only in one direction.

The longitudinal stresses are correctly distributed over the ship's height. In the double bottom we find the maximum compression stress and at the hatch coaming we find the maximum tensile stress (see figure 4.12). The maximum tensile and compression stress is equal to the stress calculated with the beam theory from the pure beam calculation.

The load application is correct and the constrained equations introduce the global forces and moments into the shell model. The equilibrium of forces is fulfilled and the contact forces are equal to the reaction forces given in $e 4$.

The trim of the vessel during receding tide is also reproduced. The data of the trim are given in $e 4$ for a rigid vessel without bending (see subsection 3.1.1). The trim from $e 4$ is compared to the values of Ha7p2el. The displacement at FP is slightly higher whereas the aft body emerges more for the rigid vessel compared to Ha7p2el. The trim calculated in $e 4$ is overestimated at all water levels because of the rigid material model. The shell section of Ha7p2el bends around the ground. It forms a sharp bend in the displacement curve and the displacement of the aft body is attenuated.

The buoyancy forces are based on the floating condition of a rigid vessel (see section 3). At the moment it is not possible to calculate the new floating condition for a flexible vessel depending on the grounding area and the tide level with $e 4$. There are no other ship line based tools known to generate the new buoyancy forces due to grounding and a flexible vessel. If the buoyancy forces are applied correctly the method would need multiple iteration loops. The inaccuracy is accepted in favour of a simple and fast method. In addition, it is shown that different buoyancy forces have a insignificant influence on the ultimate hull girder strength (see subsection 4.3.3). Furthermore, the assumption is conservative because now less buoyancy forces are assumed in the aft body. In fact for a flexible ship the buoyancy forces are higher at the stern and that would counter the light ship and dead weight.

\subsubsection{Soft ground model}

One oedometer and one triaxial test are calculated in $L S-D Y N A$ with the sand parameters given in table 4.3. The above described methodology is used to guarantee the behaviour of soil (implicit pre-step, explicit solution). The verification cases are also calculated with the FE program $A B A Q U S$. The results of $A B A Q U S$ are kindly provided by the Institute of Geotechnical Engineering and Construction Management.

The configuration of the oedometer and triaxial test are shown in figure 4.9. A cubic element is taken because the sandbank is meshed with such element geometries. There are no different stresses at the corners of the element. In each case one volume element is modelled and loaded with gravity in the pre-calculation step. The bottom nodes are 

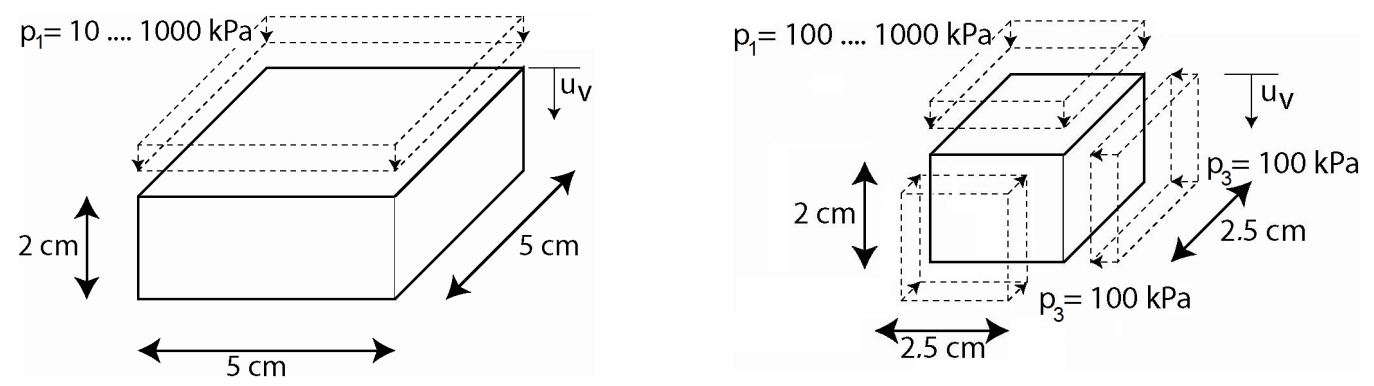

Figure 4.9.: Oedometer and triaxial test configuration.

fixed in vertical direction (z). For the oedometer test all nodes are also restricted in both horizontal directions $(\mathrm{x}, \mathrm{y})$. In the triaxial test the loading sides can slide. The pressures $p_{1}$ is applied in vertical direction. It starts with $10 \mathrm{kPa}$ and is increased up to $1000 \mathrm{kPa}$ for the oedometer test. The triaxial test is calculated for three different starting pressures $p_{3}$ $=50,100$ and $200 \mathrm{kPa}$ which are applied constantly to the sides as support.

In both FE programs the same results are achieved. Therefore only the results from $L S$ DYNA are presented.

The correct preloaded state can be checked via the vertical $\sigma_{1}^{\prime}$ and horizontal stresses $\sigma_{3}^{\prime}$. They depend on the height and the buoyant unit weight of sand (see eq. 4.5 ):

$$
\begin{aligned}
& \sigma_{1}^{\prime}=\gamma^{\prime} \cdot z \\
& \sigma_{3}^{\prime}=0.5 \cdot \sigma_{1}^{\prime}
\end{aligned}
$$

Stresses are calculated at one point in the middle of the element because of the singlepoint integration. Thus the initial stresses due to gravity are averaged over the height of the elements. In both tests the initial stresses are achieved for $h=1 \mathrm{~cm}, \sigma_{1}^{\prime}$ has to be -110 $P a$ and $\sigma_{3}^{\prime}=-55 P a$ according to equation (4.11). Exactly those values are found after the pre-step.

A simple stress-strain diagram of the oedometer test is shown in appendix B.4. The curves start at $\sigma_{1}^{\prime}=-110 P a$ due to the initial condition. After the solution the horizontal stresses equal the horizontal pressure that was loaded onto the sand.

For the triaxial tests the p'-q-diagram is given. The ordinate is half of the principle stress difference and the abscissa is half of the effective principle stress sum. The three calculations are shown in figure 4.10. The maximum values of $\sigma_{1}$ that the soil can carry are connected with a straight line, according to DIN 18137-2 [13]. The angle between the straight line and the $\mathrm{x}$-axis is called $\alpha^{\prime}$. Via $\alpha^{\prime}$ the effective angle of friction can be determined.

$$
\sin \left(\varphi^{\prime}\right)=\tan \left(\alpha^{\prime}\right)
$$

For the recalculated triaxial tests $\tan \left(\alpha^{\prime}\right)$ equals 0.574 . The effective angle of friction is $35^{\circ}$ after equation (4.12), which is the value previously defined. 


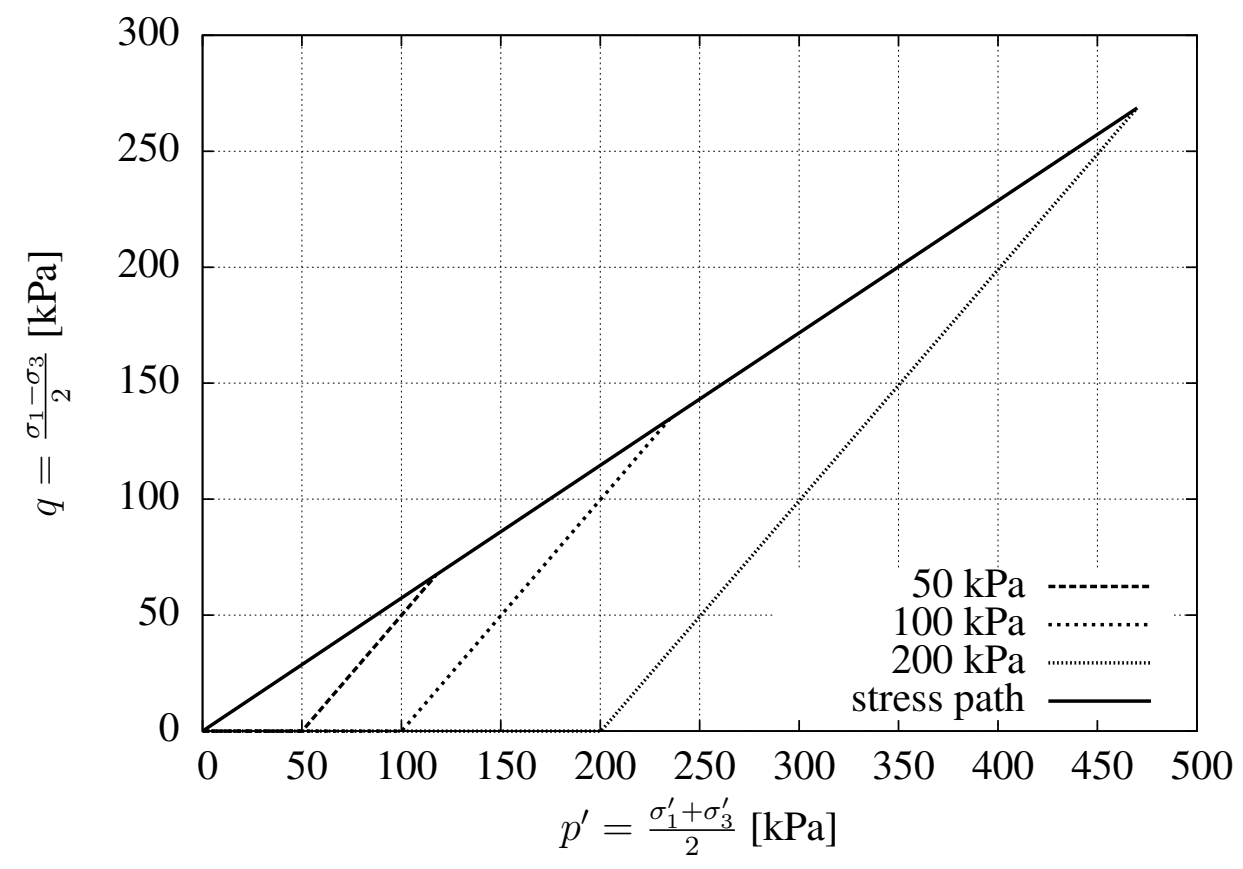

Figure 4.10.: Stess path of triaxial test.

The chosen soft ground model correctly represents the soil characteristics according to equation (4.4). The effect of stresses on the strength of soil are considered. The initial stresses are reproduced.

\subsection{Specification of calculation parameters}

All relevant parameters are listed in table 4.4 in the same order in which they are discussed now. The choice of some parameters as element type is the result of weighting up their influence on the results and on the calculation time. If no modifications are made, the calculations are very expensive. For other parameters, e.g. failure criterion, there are different possibilities that need to be evaluated.

Table 4.4.: Calculation parameters.

\begin{tabular}{ll}
\hline speed of load increase & 10 seconds per tide metre \\
time step size & $1.98 \mathrm{E}-5 \mathrm{~s}$ (mass scaling) \\
buoyancy at contact area & $\begin{array}{l}\text { constant: buoyancy deleted at initial contact area } \\
\text { element type }\end{array}$ \\
type 2 \\
failure criterion & according to Peschmann [54] \\
stress-strain curve & see figure 4.4, material type 123 \\
initial deformation & no initial deformation, welded joints not modelled \\
soft ground parameter & see table 4.3 \\
\hline
\end{tabular}


The different calculation parameters are analysed at the midship section for a rigid ground Ha7p2, because it is $5 \mathrm{~h}$ faster than Sa7p2. The average calculation time of model Ha7p2 is 50 hours (using 4 cpu of cluster with 8 cores and 48 GB RAM). Primarily the differences are compared for the total bending moment of the cross section at $147 \mathrm{~m} \mathrm{AP}$ and the collapse mode to determine the hull capacity.

At the beginning of each subsection the executed calculations are listed in a table.

\subsubsection{Load increase}

Table 4.5.: Calculations with different load increases.

\begin{tabular}{lr}
\hline case & increase \\
\hline li30 & 30 s per tide metre \\
li15 & 15 s per tide metre \\
Ha7p2 & 10 s per tide metre \\
li5 & 5 s per tide metre \\
\hline
\end{tabular}

Within the explicit solver any time-dependent values can play a role. Furthermore applying loads is not a usual procedure in explicit solutions. To check the influences of the load increase speed on the simulation results and to exclude dynamic effects different speeds are analysed (see table 4.5). The computational effort directly depends on the load increase, e.g. case li30 needs to be simulated $150 \mathrm{~s}$ whereas case Ha7p2 only needs $50 \mathrm{~s}$. The total calculation time has no linear dependency on the increase.

The differences in the bending moment for cases li30, li15 and Ha7p2 are very small, whereby case li5 gives different results. The first eigenfrequency of the whole system is $2 \mathrm{~Hz}$. It is clearly necessary in an explicit solution to increase the external loads more slowly than one cycle period; $5 \mathrm{~s}$ is obviously to short. If the bending moments and shear forces of case li5 are checked at the beam elements it becomes clear that the applied loads are not fully acting. The system needs more time until the forces are active.

A load increase within 10 s provides a reasonable result and has a comparably short computation time.

\subsubsection{Time step size}

Table 4.6.: Calculations with different time step sizes.

\begin{tabular}{llrrr}
\hline case & time step $[\mathrm{s}]$ & area $\left[\mathrm{m}^{2}\right]$ & contact stiffness & damping \\
\hline t1a1 & $\Delta \mathrm{t}=1.97 \mathrm{E}-6$ (auto) & $\mathrm{A}=4.8$ & penalty & no \\
t1a1d & $\Delta \mathrm{t}=1.97 \mathrm{E}-6$ (auto) & $\mathrm{A}=4.8$ & penalty & yes \\
t1a2 & $\Delta \mathrm{t}=4.90 \mathrm{E}-6$ (auto) & $\mathrm{A}=480$ & penalty & no \\
t2a2 & $\Delta \mathrm{t}=1.98 \mathrm{E}-5$ (mass scaling) & $\mathrm{A}=480$ & penalty & no \\
Ha7p2 & $\Delta \mathrm{t}=1.98 \mathrm{E}-5$ (mass scaling) & $\mathrm{A}=480$ & soft constraint & no \\
\hline
\end{tabular}


The time step size is 1.97 E-6 s without any adjustments (t1a1). It is very small and does not change during the solution. The size and thereby the overall calculation time needs to be reduced by coincidentally guaranteeing the correct solution to the problem. The explicit solver has a time step problematic (see section 2). The integration method is stable if the global time step size is smaller than the critical time step. The critical time step depends on the highest eigenfrequency of the system and is given without viscous damping in the following equation:

$$
\Delta t \leq \Delta t^{c r i t}=\frac{2}{\omega_{\max }}
$$

The global time step size is the minimum value out of all finite elements. It is determined after each time step with the Courant-Levy stability criterion (see LS-DYNA theoretical manual [37]) given in equation (4.14).

$$
\Delta t=0.9 \cdot \frac{\ell}{c}
$$

The characteristic element length $\ell$ is the shortest element edge length. For the chosen element types the determination of the sound speed $c$ depends on Young's modulus, Poisson's ratio and mass density:

$$
\begin{aligned}
& \text { solid element: } c=\sqrt{\frac{E(1-\nu)}{\rho(1+\nu)(1-2 \nu)}} \\
& \text { shell element: } c=\sqrt{\frac{E}{\rho\left(1-\nu^{2}\right)}} \\
& \text { beam element: } c=\sqrt{\frac{E}{\rho}}
\end{aligned}
$$

For the used beam elements the global time step size given in equation (4.14) and (4.15) is not used because the bending-related time step size is smaller. This is calculated by multiplying equation (4.14) with factor fact (see equation (4.16)). Thus not only the element size but also the cross section area $A$ and moment of inertia $I$ directly influence the time step size.

$$
\text { fact }=1.8 \cdot\left(\sqrt{3 I\left[\frac{3}{12 I+A \ell^{2}}+\frac{1}{A \ell^{2}}\right]}\right)^{-1}
$$

A good way to enlarge the time step size is to increase the mass density of the critical elements, so-called mass scaling. Mass scaling influences the amount of inertial mass but not the gravitational mass. The new time step size is provided by the user and will not change during the solution. The new mass density of each critical element is achieved by solving equation (4.14) for the density. Doubling the time step size quadruplicates the 
density and therefore mass scaling has to be used carefully.

In addition to the very small time step the model oscillates. Especially the beam elements oscillate because of the relative low mass. They have a relative small area $A$ of maximal $4.8 \mathrm{~m}^{2}$ compared to the moment of inertia. If the area $A$ is increased to $480 \mathrm{~m}^{2}$ $(H \cdot B / 2)$ there are two positive effects: the model no longer oscillates and the time step size is more than doubled to $4.9 \mathrm{E}-6 \mathrm{~s}$ (t1a2). The bending moment of calculation t1a1 and $\mathbf{t 1 a 2}$ differ by $0.9 \%$.

The other way to reduce the oscillations is to damp the system with a mass proportional Rayleigh-damping. This type of damping only effects the mass matrix, damps the lower eigenfrequencies and thus reduces global speeds. The mass proportional damping does not influence the time step, because the highest eigenfrequency is almost unchanged. However damping should not be used if not absolutely necessary, because it could distort the results. Furthermore, the calculation time raises with the use of damping. In different simulations the best damping coefficient was found to be 0.2 and this is used in calculation t1a1d. Although t1a1d gives similar results as t1a2 the latter is the better choice concerning damping.

If the time step size is set to $1.98 \mathrm{E}-5 \mathrm{~s}(\mathbf{t} 2 \mathbf{a} 2)$ the calculation is 10 times faster than t1a2. Thereby the total mass is increased by $2.33 \%$. The critical elements that are loaded with added mass are around the manholes.

Increasing the time step size a little bit more the added mass becomes disproportional high. Then many elements would reach a critical size resulting in a high added mass.

The time step size has a small influence on the results. The maximum deviation between case $\mathbf{t 1 a} 2$ and $\mathbf{t} \mathbf{2 a} 2$ is less than $1 \%$ for the bending moment at $3 \mathrm{~m}$ of surface drawdown.

The provided time step size of $1.98 \mathrm{E}-5 \mathrm{~s}$ is higher than the size recommended due to the contact if the default penalty method is used. The contact stiffness depends again on the size of the elements involved in the contact. Here all elements are involved in the contact condition (see section 4.1). The recommended time step size for the implemented contact should be $1.16 \mathrm{E}-5 \mathrm{~s}$ to avoid contact instabilities. Thus the soft constraint option is chosen (Ha7p2). The contact stiffness $k$ is thereby determined as needed for the specified time step size and multiplied by the factor sof scl (see equation (4.17)).

$$
k=\operatorname{sofscl} \cdot \frac{m_{s}}{\Delta t^{2}}
$$

Both methods to control the contact stiffness, t2a2 versus Ha7p2, give exactly the same results and need the same calculation time. The penalty method does not lead to instabilities even if the time step size is larger than the recommended size. However the soft constraint method is chosen. 


\subsubsection{Buoyancy}

Table 4.7.: Calculations with different buoyancy applications.

\begin{tabular}{ll}
\hline case & type \\
\hline Ha7p2 & buoyancy at contact \\
Ha7p2B & no buoyancy at contact \\
\hline
\end{tabular}

In the initial floating condition the buoyancy is applied at all vessel lengths. If the water level is reduced contact with the ground arises. The contact area increases with receding tide. There are no buoyancy forces at the contact area. For simplification the buoyancy is only deleted at the initial contact area (between 146 and $148 \mathrm{~m} \mathrm{AP)}$ ).

The changing length of the contact area is known from case Ha7p2 and thus the buoyancy can be correctly deleted for the next case Ha7p2B. The enlarging contact area length is given in table 4.9. When the water level is reduced by $5 \mathrm{~m}$ the three modelled cargo holds are in contact with the ground.

The bending moment distribution is almost the same for both cases. Up to $1 \mathrm{~m}$ of receding tide the distribution of case Ha7p2 is shifted by $1 \%$ in the direction of the surface drawdown. Thus this model suffers a little later the same loads as case Ha7p2B. The difference is so small that it is not necessary to consider the lost buoyancy at the contact area.

\subsubsection{Element type and failure criterion}

Table 4.8.: Calculations with different element types and failure criterion.

\begin{tabular}{lrl}
\hline case & type & criterion \\
\hline Ha7p2 & 2 & Peschmann [54] criterion \\
e16f1 & 16 & Peschmann [54] criterion \\
e16f2 & 16 & no failure criterion \\
e16f3 & 2 & GL criterion \\
\hline
\end{tabular}

Using under-integrated shell or volume elements (single point integration) can lead to so-called hourglass modes. Hourglass modes are non-physical, zero-energy modes of deformation that produce zero strain and no stress due to a rank drop in the stiffness matrix. Hourglassing can be introduced by single loads or contact forces at single nodes. There are two possibilities in LS-DYNA to control hourglassing. One is to automatically stabilise the single integrated element against zero-energy deformation. A way to entirely eliminate hourglassing is to switch to fully-integrated element formulations. But there is a downside to this approach. They are much more expensive than the single point integrated elements. Secondly, they are much more unstable in large deformation applications. And third, they have some tendency to behave too stiffly in applications where the element shape is poor. 
Hourglass modes do not occur in case Ha7p2. The hourglass control is sufficient and critical elements are deleted because of the failure criterion before they have zero-energy modes. If the model is simulated without the failure criterion houglassing becomes a problem and the fully integrated element type 16 is chosen for the cargo hold section (e16f2). Type 16 is a fully integrated formulation with enhanced assumed strain (EAS) that is an extended distortion approach to compensate shear stiffening. To compare the different failure criteria a third case is needed. To exclude the influence of the different element types case e16f1 has the same failure criterion as $\mathbf{H a 7 p 2}$ and the element type of e16f2. In addition, the so-called $G L$ failure criterion is tested, whereby the critical strain $\epsilon_{k}$ also depends on the element thickness $t$ and the element edge length $\ell$. For more details reference is made to Egge et al. [15].

$$
\epsilon_{k}=0.056+0.54 \cdot \frac{t}{\ell}
$$

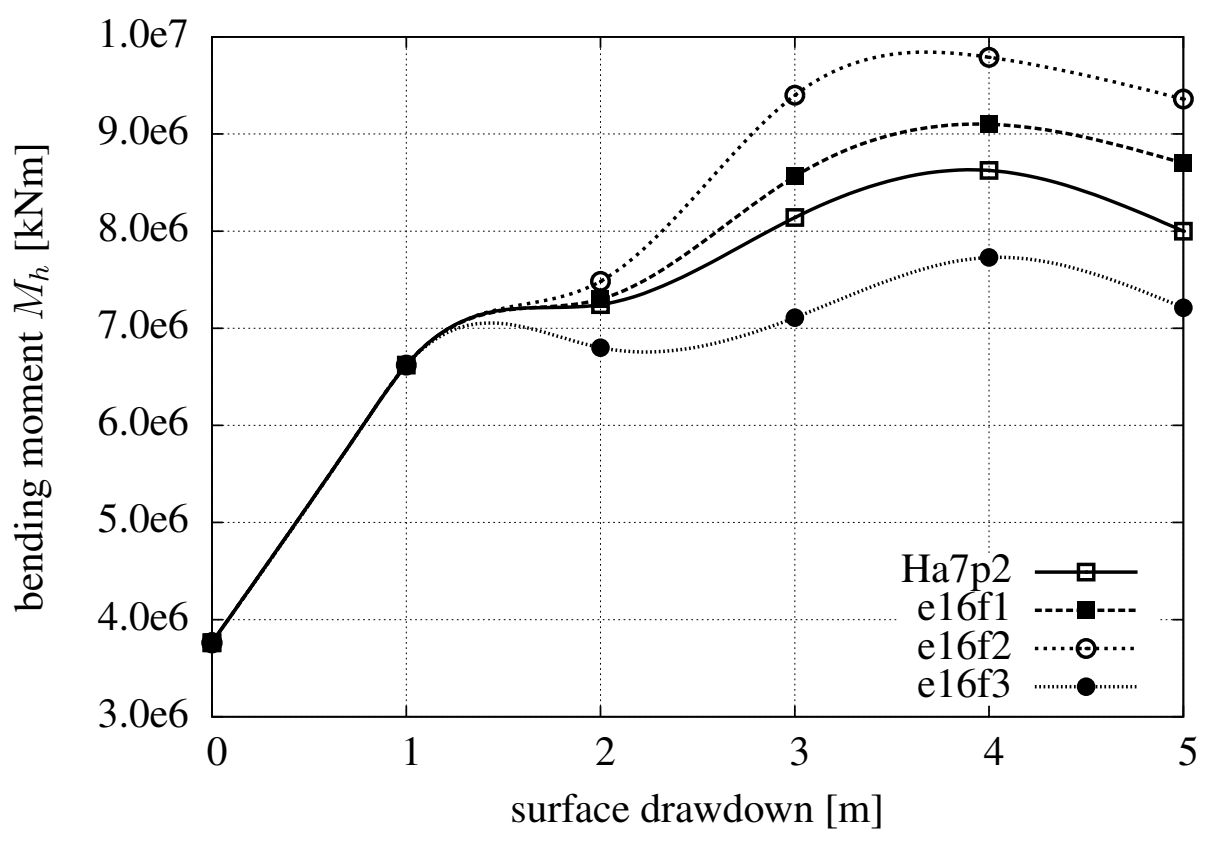

Figure 4.11.: Ha7p2, e16f1, e16f2 and e16f3: bending moment $M_{h}$ at $147 \mathrm{~m} \mathrm{AP.}$

Figure 4.11 presents the ultimate strength measured at cross section $147 \mathrm{~m}$ AP of the four cases. Between 0 and $1 \mathrm{~m}$ of surface drawdown the whole model is in the elastic range of the material and all cases have the same total bending moment. After $1 \mathrm{~m}$ of water level reduction the vessel enters the plasticity range and the different parameters give other results. The course of the ultimate strength is the same. The highest bending moment is achieved by case e16f 2 and the lowest by e16f3. The distribution of e16f1 and Ha7p2 lie in between. Case e16f1 and e16f2 are stiffer than Ha7p2 or e16f3 and can sustain higher loads. The third downside mentioned above increases the stiffness of the system when element type 16 is chosen. Without failure the loads are distributed to more elements and transmission to intact structural elements is better. Therefore, cases Ha7p2 and $\mathbf{e 1 6 f 3}$ are conservative considerations. e16f3 can sustain lower moments than Ha7p2. 
Elements are deleted earlier because of the smaller critical strains (compare equation (4.1) and (4.18)).

Using shell type 16 leads to longer calculation times. For example, e16f2 needs twice the time of Ha7p2.

Of all parameters, the element type and failure criterion have the greatest influence on the results. The quality of the results is the same and only the quantity varies. Ha7p2 is chosen for the upcoming variations because its results lie in between the other cases. It is cost-efficient and excludes the effects described above.

\subsubsection{Summary of calculation parameters}

The system is in any case very insusceptible to modification. The chosen modification to achieve a faster calculation time does not cause a loss in the quality of the results. The parameters used for further calculations are already given in table 4.4.

\subsection{Comparison with double T-beam}

A double T-beam, called Tbeam, is calculated with the length, height and the loading condition of Ha7p2. The results of the calculation are useful to check the plasticity effects of the simulation method. To compare the results to the theory of ultimate load-bearing capacity for simple structures a failure criterion is not used. Thus the vessel is also calculated without any failure criterion (e16f2, see section 4.3.4).

An unsymmetrical double T-beam is modelled with the above described shell elements, material models and boundary conditions. Thus strain hardening and different yield stresses are taken into account. The total length and height are the same as that of the vessel. The flanges are very short to exclude the effect of effective breadth because the double bottom in $\mathbf{e 1 6 f 2}$ has also no effective breadth. The plate thickness are chosen to achieve best agreement with the cross-section area and the elastic/plastic section modulus.

The longitudinal stress distribution at cross section $147 \mathrm{~m}$ AP of Tbeam (in grey) and e16f2 (in black) are compared in figure 4.12 for the initial floating condition $(0 \mathrm{~m}), 1,3$ and $5 \mathrm{~m}$ of surface drawdown. The different yield stresses and their distribution in the models over the height are given in colour: yellow for 235, green for 315 and red for 355 $\mathrm{N} / \mathrm{mm}^{2}$. The thickness of the colour scale represents the amount of structural elements with the specific yield stress. So, most elements have a yield stress of $315 \mathrm{~N} / \mathrm{mm}^{2}$. Furthermore, the height of the structural elements stringer s1, s3 and inner bottom ib as well as the elastic (e.n.a.) and plastic neural axis (p.n.a.) are given. The results of Tbeam are given in grey with many sampling points and the results of $\mathbf{e 1 6} \mathbf{2} 2$ are given in black with dots for the sampling points.

Until $1 \mathrm{~m}$ of receding tide the stress distribution is linear and the stresses are below the yield stresses. Because of the three different yield stresses the straight line has different 


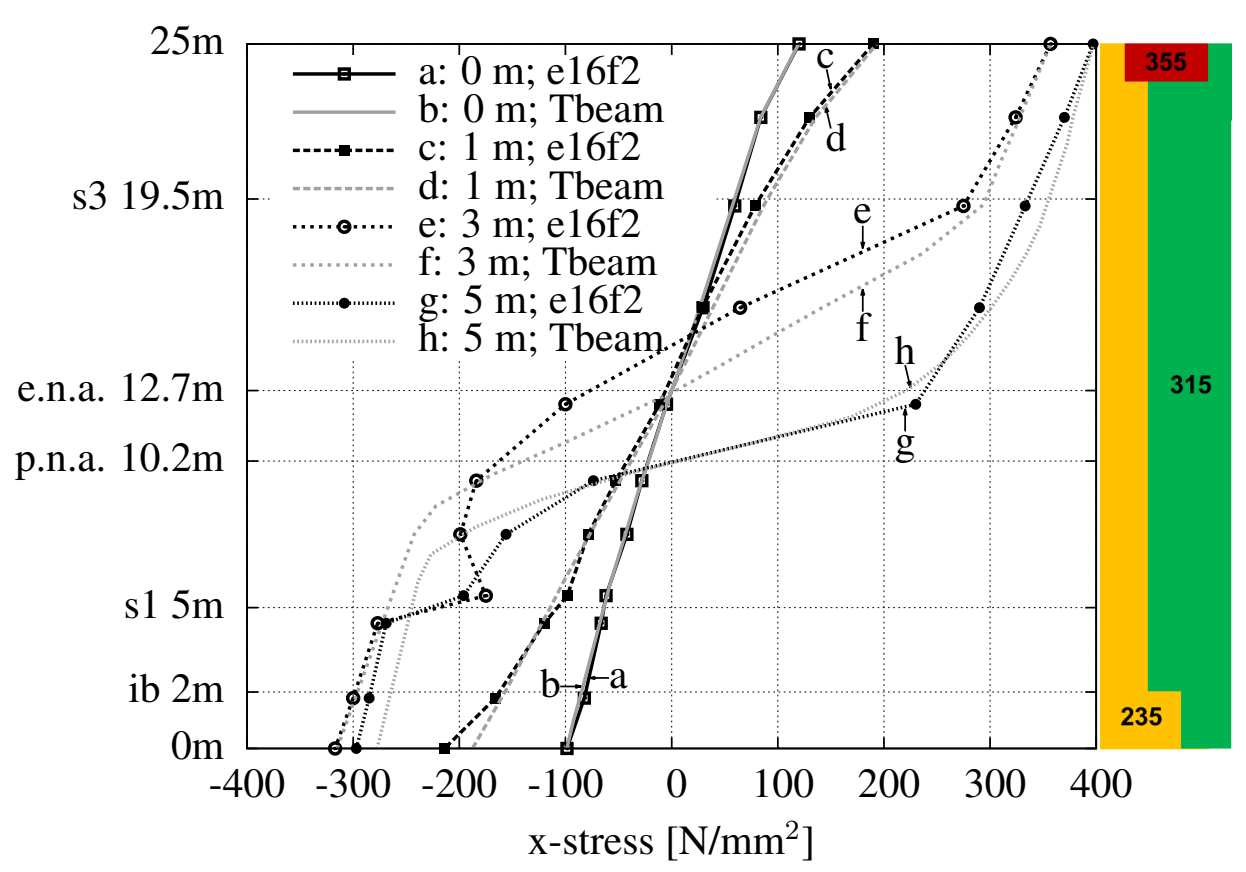

Figure 4.12.: e16f2 and Tbeam: longitudinal stress distribution at $147 \mathrm{~m} \mathrm{AP.}$

gradients. At $3 \mathrm{~m}$ of water level reduction the cross section starts to plasticise and zerocrossing is above the elastic neutral axis for case $\mathbf{e 1 6 f} \mathbf{2}$, whereas for Tbeam the axis does not move. In both cases the neutral axis slides down with receding tide. After buckling in the double bottom (see figure 4.13) the neutral axis moves towards the tension side and further loads can be sustained until the deck yields. There is a reserve strength beyond collapse of the compression side i.e. the double bottom. This phenomenon is also reported by Rutherford and Caldwell [64] and Paik and Mansour [50] and comparable results for a passenger ship are also presented in a benchmark study published in Committee III.1 [9]. For $5 \mathrm{~m}$ of surface drawdown the height of the plastic neutral axis is the same as the one achieved by the following simplified formula given by Lehmann and Zhang [36].

$$
\text { p.n.a. }=\frac{A_{1}-A_{2}+A_{s t}}{2 \cdot s_{s t}}=10.2 \mathrm{~m}
$$

Equation (4.19) applies for unsymmetrical double T-girders and $A_{1}$ is the area of the top flanges, $A_{2}$ the area of the lower flanges, $A_{s t}$ and $s_{s t}$ the area/thickness of the web. If p.n.a. is calculated for the cross section of e16f2 at $147 \mathrm{~m} \mathrm{AP}$ by hand with the method also proposed by Lehmann and Zhang [36] the same result is achieved.

The stress distribution for $5 \mathrm{~m}$ of receding tide of Tbeam is suitable with the plastic hinge theory. It is not rectangular above and below the plastic neutral axis and the maximum stresses in top and bottom are higher than the predominant yield stresses due to the different yield stresses.

The differences between Tbeam and e16f 2 are because of the different cross sections. The joint of the side structure to the bottom structure (called stringer s1) is $5 \mathrm{~m}$ over the 
base line (see figure 4.13) which leads to a sharp change of the stress distribution for 3 and $5 \mathrm{~m}$ of tidal range. Even for the initial floating condition and a surface drawdown of $1 \mathrm{~m}$ it is seen. The inner double bottom ib also has an influence on the distribution. The influence of stringer $\mathrm{s} 3$ can be identified in the stress distribution for $3 \mathrm{~m}$ of water level reduction.

Figure 4.13 shows the deformation at cross section $147 \mathrm{~m}$ AP for e16f2. The buckling of the double bottom structure until stringer $\mathrm{s} 3$ can be seen. The immense buckling phenomenon explains why $\mathbf{e 1 6} \mathbf{2} \mathbf{2}$ reaches higher stresses at the base line as Tbeam for $5 \mathrm{~m}$ of receding tide.

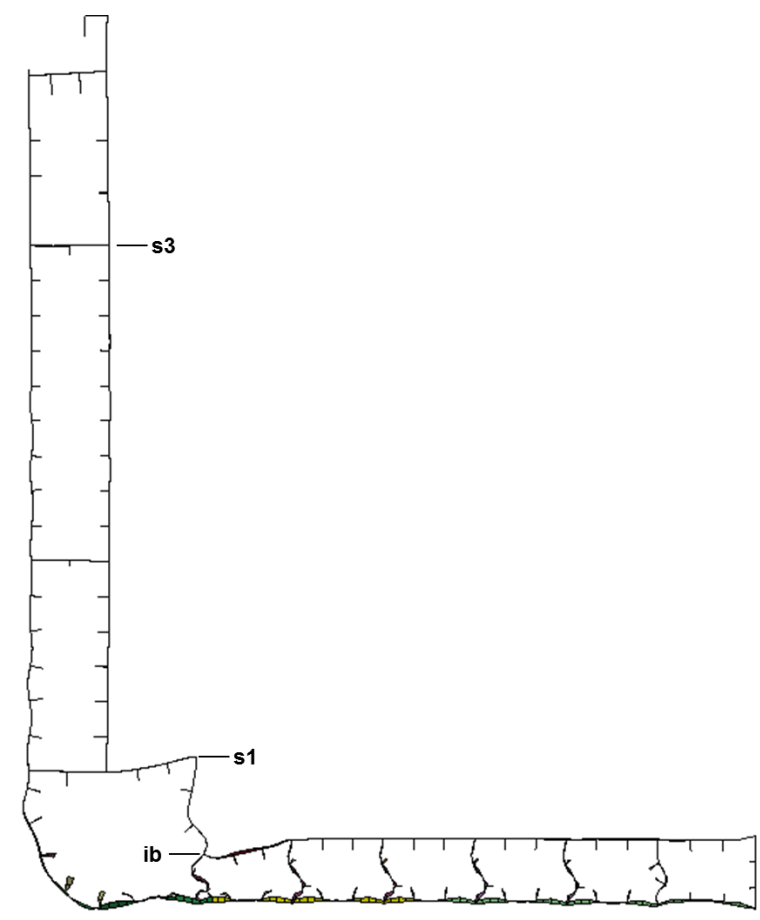

Figure 4.13.: e16f2: deformation of cross section $147 \mathrm{~m} \mathrm{AP.}$

The Tbeam calculation provides good results with respect to the stress distribution and verifies model e16f 2 beyond the elastic beam theory. But to analyse all effects of stranding to a ship structure a T-beam model is not sufficient.

\subsection{Results of the midship scenario}

The results of the midship scenario are given in detail and those of the aft and the fore body scenarios are given in brief at the end of the section. Most phenomena are discussed using ground geometry $\mathbf{a 7 p 2}$. Bending moment and shear force curves, ultimate strengths, stresses, collapse modes and the influence of ground parameters and geometries are shown.

Furthermore the stranding incident of the container vessel Fowairet is recalculated with the presented method and the results are compared. 


\subsubsection{Bending moment and shear force curves}

Figure 4.14 shows the bending moment and shear forces of case Sa7p2 over the ship length for 1, 3 and $5 \mathrm{~m}$ of tide level. In all graphs the results of case $\mathbf{A} \mathbf{1}$ of the pure beam calculation are shown as a reference.
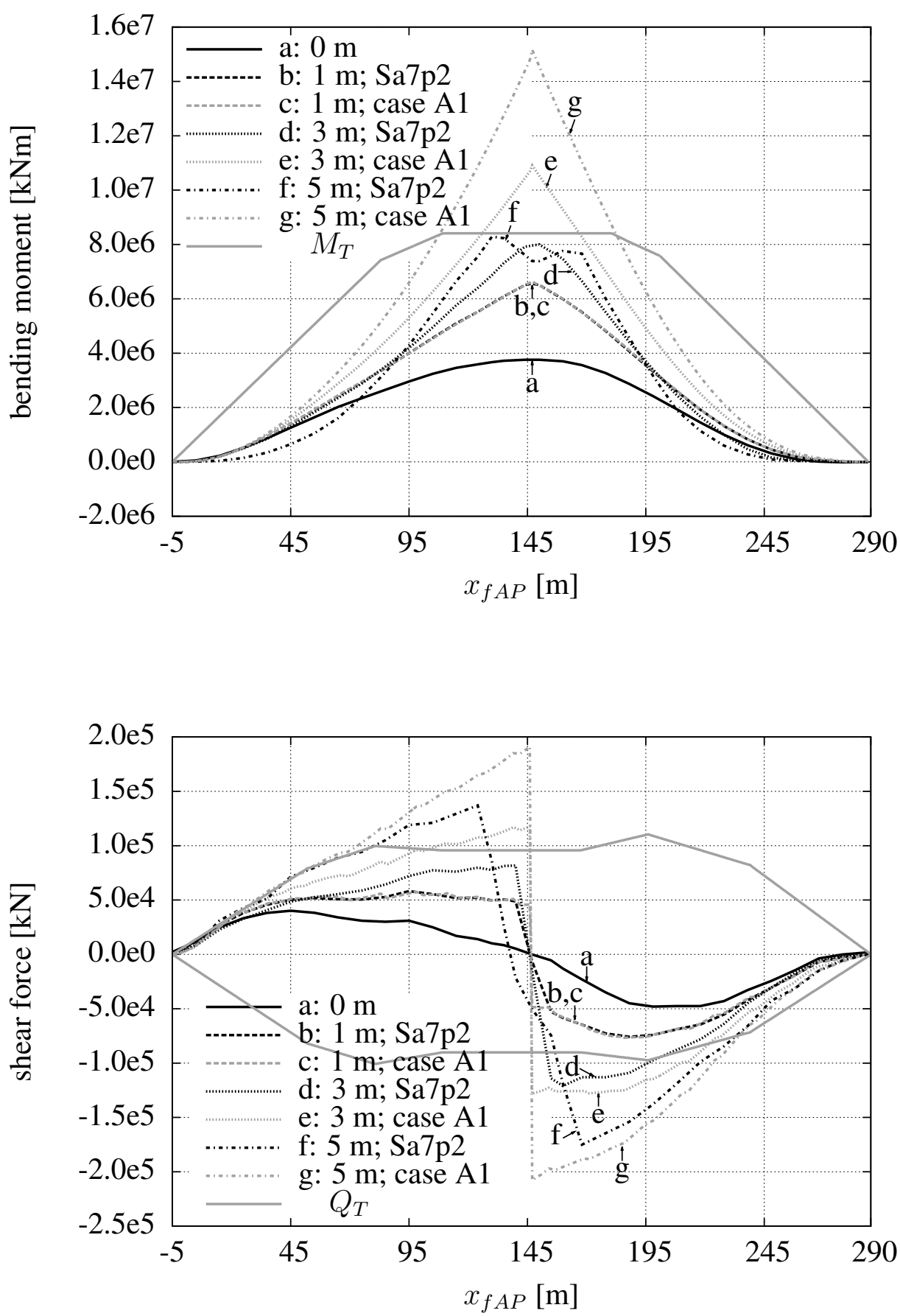

Figure 4.14.: Sa7p2 and case A1: bending moments and shear forces.

For $1 \mathrm{~m}$ of surface drawdawn the bending moment and shear force curves are almost 
the same as for the pure beam calculation. The stresses are in between the elastic range, being smaller than the yield stresses. The model behaves according to the elastic beam theory.

The differences are at the stranding point/area according to the differences between

Ha7p2el and case A1 described in subsection 4.2.1. In simulation Sa7p2 the vessel already lies on an area of $9.3 \mathrm{~m}$, whereas the beam has one contact point.

The contact area of Sa7p2 increases with receding water. The effect can be seen very well for the shear force curve. The moments are clearly smaller than for the pure beam case. The structure can not absorb the high forces and moments as predicted by the elastic beam theory and the difference in the shear force capacity is smaller than for the bending moments. The bending moments of $\mathbf{S a 7 p 2}$ stay inside the envelope of $M_{T}$. The shear forces are higher than the maximum permissible forces $Q_{T}$ up to $3 \mathrm{~m}$ of surface drawdown. For $3 \mathrm{~m}$ of receding tide the curves are qualitatively the same as for case A1. The larger contact area with a length of $16.5 \mathrm{~m}$ results in the rounded peak and in the gradient of the shear force jump. But the main difference is a result of the different material properties. As already shown in figure 4.12 the structure soon enters the plastic range. The bending moment curve for $5 \mathrm{~m}$ of surface drawdown indicates the post collapse mode where the whole shell-section is in contact with the ground.

In figure 4.15 the normalised bending moment at $147 \mathrm{~m} \mathrm{AP} M_{S a 7 p 2} / M_{c a s e}{ }_{A 1}$ is plotted over the normalised shear force jump $Q_{S a 7 p 2} / Q_{\text {case } A 1}$. To obtain this the moment/shear force jump of Sa7p2 is divided by those of case A1. The shear force jump is built up by the maximum positive and maximum negative shear force at the contact. The normalisation shows that the shear forces are better absorbed by the structure than the bending moment, especially in the post-collapse range. The quantity of the points changes if another reference, e.g. case A2, with the same contact area is taken, but the quality is the same.

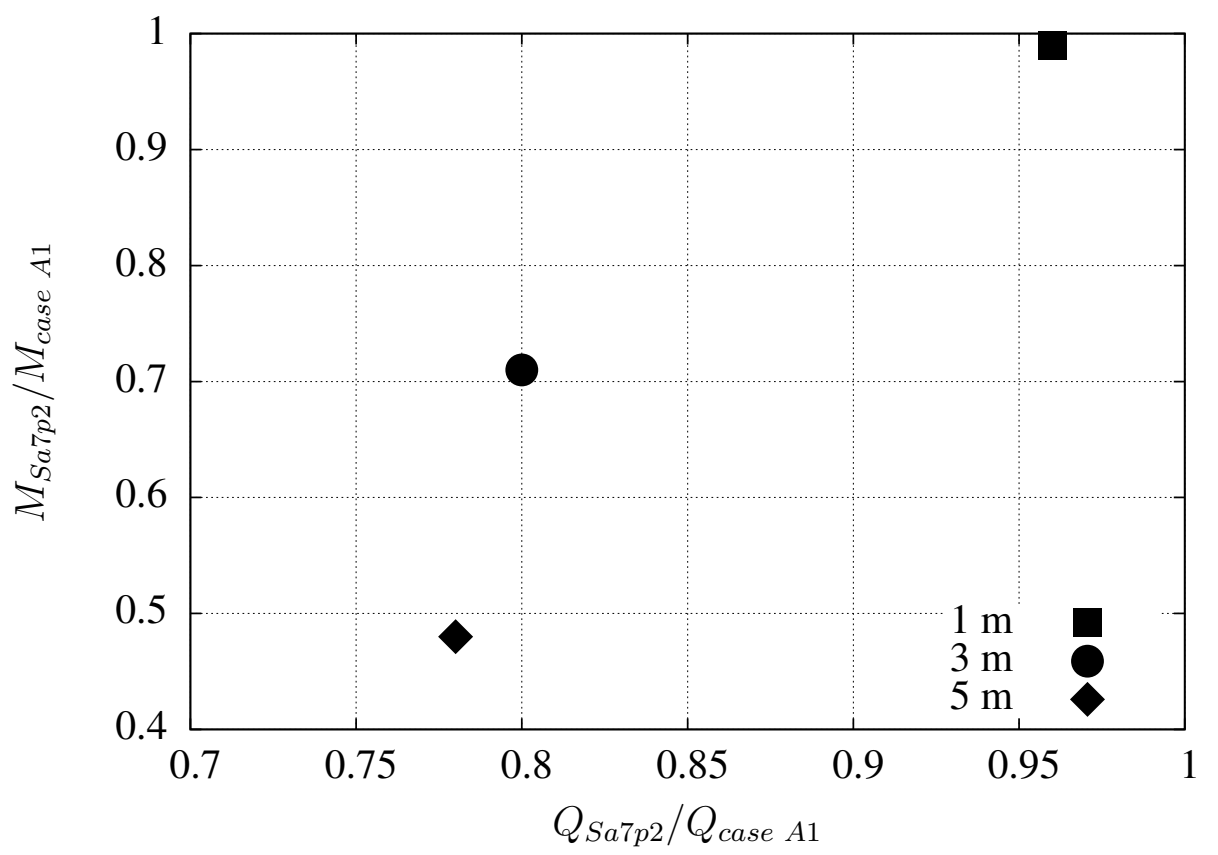

Figure 4.15.: Sa7p2: normalised moments and shear forces at $147 \mathrm{~m}$ AP. 


\subsubsection{Bending moment $M_{h}$}

Figure 4.16 and 4.17 show the total bending moments and shear forces over the water level reduction of calculation Ha7p2 and Sa7p2 measured at all intact elements of cross section 147 m AP (see equation (4.9)). It implies how much of the globally applied moments the cross section can bear. Again the bending moment of case A1 is shown as a reference. For the shear forces there is no reference line from the beam calculation because the cross section lies at the shear force jump. Thus calculation Ha7p2el is taken as a reference case. Ha7p2el includes the effects of trim, bending and increasing contact area which are relevant for the shear forces at that section (see subsection 4.2.1).

In both stranding simulations the moments lie below the pure beam results after $1 \mathrm{~m}$ of water level reduction. The cross section cannot absorb such a high bending moment as that predicted by the elastic beam theory. The highest yield stress of $355 \mathrm{~N} / \mathrm{mm}^{2}$ is already exceeded in positive and negative direction for $1.2 \mathrm{~m} \mathrm{(Sa7p2)/1.4} \mathrm{m}(\mathbf{H a} 7 \mathbf{p 2})$ of surface drawdown. The first elements start to fail at $1.5 \mathrm{~m}$ of water level reduction in both simulations. The ultimate hull girder strength is reached shortly after $3 \mathrm{~m}$ of receding tide for Sa7p2 and at $4 \mathrm{~m}$ for Ha7p2. Ha7p2 reaches even higher moments and thus is not the conservative case. After the ultimate strength is reached the vessel is still in an equilibrium position due to the support of the ground. The ultimate strength of the section during stranding in both cases is around $30 \%$ less than predicted by the classification societies (see subsection 2.4.1).

For $1 \mathrm{~m}$ of surface drawdawn all bending moments are almost the same. The differences are again due to the different contact areas (see subsection 4.5.1 and table 4.9). The moment of Ha7p2 lies between Sa7p2 and case A1. The longitudinal stresses are below the yield stresses and thus the models are still in the elastic range of the true stress-strain curves.

When the water level is reduced further, the contact area and the differences to the ideal elastic beam theory increase. After $1.2 \mathrm{~m}$ or $1.4 \mathrm{~m}$ of surface drawdown calculations Sa7p2 and Ha7p2 are outside the ideal elastic range. Now the length of the contact area has the contrary effect. The longer the contact area the higher the moments that can be absorbed by the structure (see table 4.9 and figure 4.16). For $3 \mathrm{~m}$ of receding tide the contact length is almost the same and so are the moments. This does not apply for $5 \mathrm{~m}$ of receding tide because both simulations are in the post-collapse mode.

The vessel trims in both simulations to the bow and so the contact area length enlarges faster in bow direction.

Table 4.9 also gives the z-displacement of the cargo hold section relative to the base line of $0 \mathrm{~m}$. The values are achieved by averaging the displacements of the double bottom at the two bulkheads. At $3 \mathrm{~m}$ of surface drawdown the bulkheads of Ha7p2 touch the ground and no more displacement is possible due to the rigid ground. In simulation Sa7p2 the vessel is constantly pushed into the soft ground. Between 4 and $5 \mathrm{~m}$ of receding tide the bulkheads are lifted. A main fold in the middle of the cargo hold lifts the midship section.

The shear forces are hard to asses at cross section $147 \mathrm{~m} \mathrm{AP}$ due to the shear force 


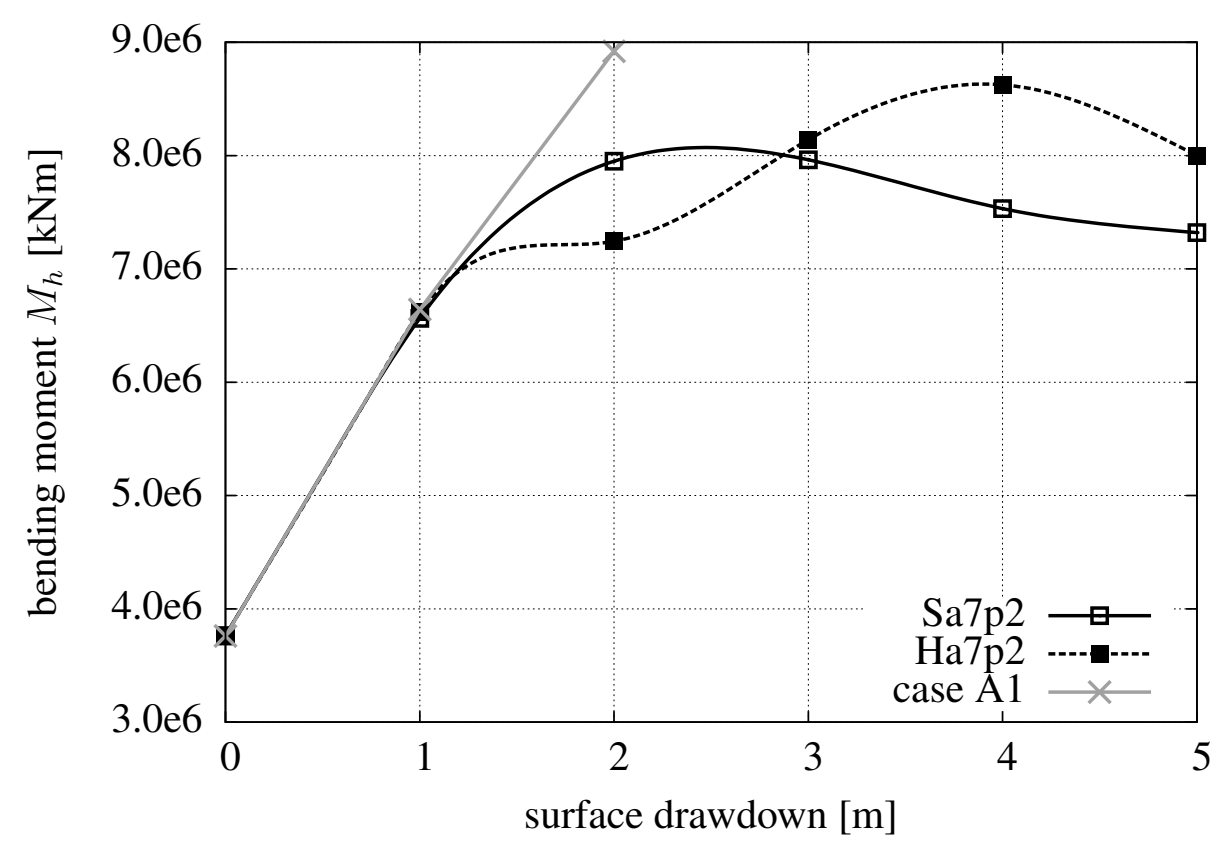

Figure 4.16.: Sa7p2 and Ha7p2: bending moment $M_{h}$ at $147 \mathrm{~m} \mathrm{AP.}$

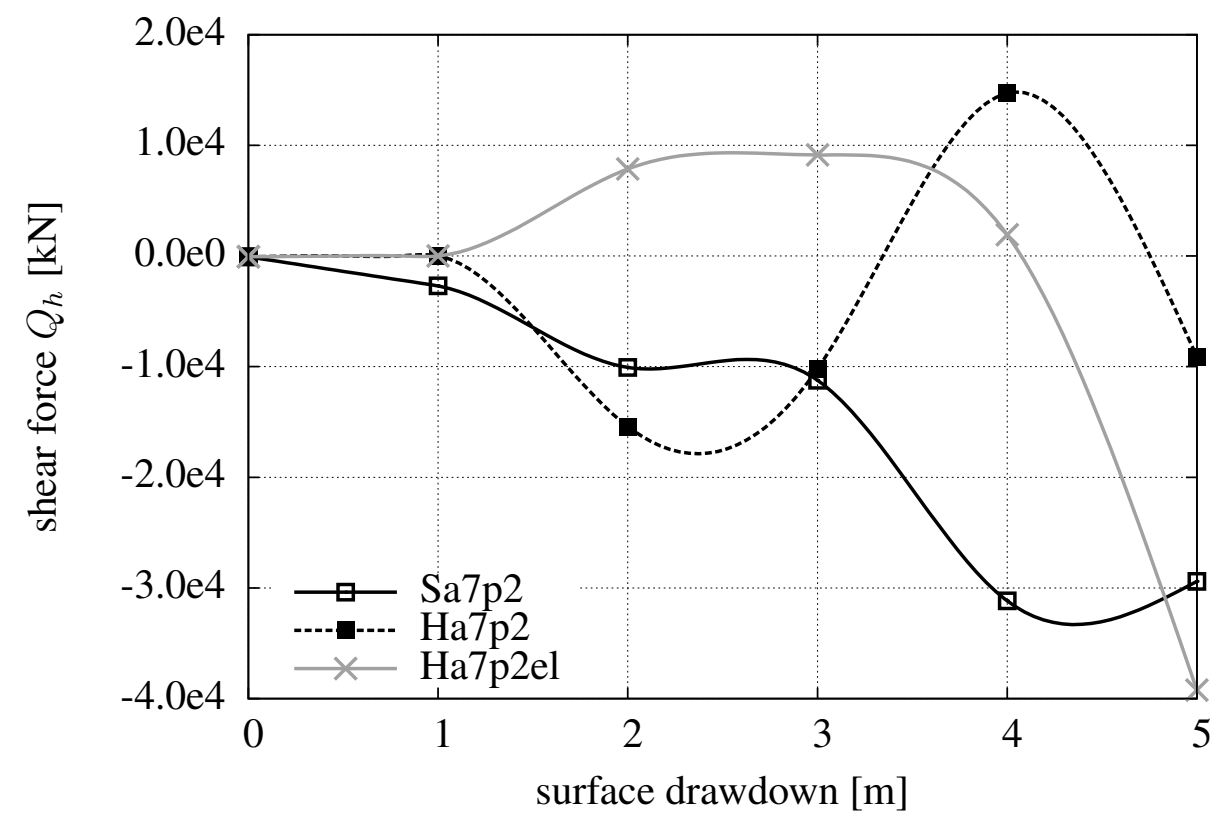

Figure 4.17.: Sa7p2 and Ha7p2: shear force $Q_{h}$ at $147 \mathrm{~m} \mathrm{AP.}$

jump. The gradient of the jump is differently increased during receding tide. The shear forces of Sa7p2 and Ha7p2 at cross section 146, 147 and $148 \mathrm{~m} \mathrm{AP}$ are also given in appendix B.4 to clarify the effect of different contact lengths. Ha7p2 and case A1 are identical up to $1 \mathrm{~m}$ of surface drawdown and Sa7p2 differs due to the different contact length. The gradient change of the curves, which is described in subsection 4.2.1 for Ha7p2el, is immediately valid for Sa7p2. Up to a surface drawdown of $1 \mathrm{~m}$ it is also 
Table 4.9.: Sa7p2 and Ha7p2: contact length and z-displacement.

\begin{tabular}{crrrr}
\hline \multirow{2}{*}{$s_{d}$} & \multicolumn{2}{c}{ Sa7p2 } & \multicolumn{2}{c}{ Ha7p2 } \\
{$[\mathrm{m}]$} & contact [m] & Z-displ. [m] & contact [m] & Z-displ. [m] \\
\hline $\mathbf{1}$ & 9.30 & -0.42 & 2.00 & -0.02 \\
$\mathbf{2}$ & 13.00 & -0.66 & 6.30 & -0.29 \\
$\mathbf{3}$ & 16.50 & -0.88 & 18.80 & -0.59 \\
$\mathbf{4}$ & 25.40 & -0.98 & 35.50 & -0.56 \\
$\mathbf{5}$ & 42.00 & -0.88 & 42.00 & -0.57 \\
\hline
\end{tabular}

valid for Ha7p2, because the contact length increases in direction of the fore body. The shear force curve of $\mathbf{S a 7 p 2}$ reflects the bending moment between 0 and $4 \mathrm{~m}$ of receding tide, whereas the shear force of Ha7p2 shows effects that are not indicated by the associated moment. In fact almost the same shear forces are found at $3 \mathrm{~m}$ of receding tide for both simulations. The outlier at $4 \mathrm{~m}$ of water level reduction arises because the double bottom at that section and between the bulkheads is suddenly lifted $0.03 \mathrm{~m}$ from the ground. Shortly afterwards it is lowered again. The midship section of Sa7p2 is lifted at $5 \mathrm{~m}$ water level reduction and thus the shear force also increases.

\subsubsection{Stress and collapse}

The following figures always show the results of Ha7p2 on the left side and the corresponding results of $\mathbf{S a 7 p 2}$ on the right side. The mesh is not shown. For a better orientation some structural elements, the centre line (CL), the bilge and $147 \mathrm{~m} \mathrm{AP}$ are marked (see table 4.10).

Table 4.10.: Structural elements marked in figure 4.18, 4.20-4.22 and 4.26- 4.27.

\begin{tabular}{llll}
\hline number & mark & \multicolumn{2}{c}{ structural element } \\
& & double bottom & side structure \\
\hline $\mathbf{1}$ & $\mathbf{b 1 / b 2}$ & bulkhead (floor) & bulkhead (web) \\
$\mathbf{2}$ & $\mathbf{f l 1 / f 1 2 / f l 3}$ & floor & web frame \\
$\mathbf{3}$ & - & bottom longitudinal frame & longitudinal frame \\
$\mathbf{4}$ & $\mathbf{s 2 / s 3}$ & longitudinal girder & stringer \\
\hline
\end{tabular}

In figure 4.18 the final collapse of $\mathbf{H a 7 p 2}$ and Sa7p2 is plotted. The deformation of the middle cargo hold is given scale of 1:1. The transverse main folds are marked in red and the longitudinal main folds/buckle fields are marked in yellow.

In both cases the structure starts to collapse at the connection of bilge plates and longitudinal girders between 1 and $2 \mathrm{~m}$ of receding tide. From there the collapse moves into the side and double bottom structure. In the double bottom there is an additional failure starting point. The longitudinal girders start to buckle around their manholes between floor fl1 and fl2, shorty after the failure of the bilge structure. At $5 \mathrm{~m}$ of surface drawdown 
there are one to two deep transverse and longitudinal folds and diverse deep buckles. The outer shell of the side structure buckles isotropically around the main fold. In the outer shell of the double bottom there are orthotropic buckles. In both simulations the main transverse fold can be compared to a plastic hinge in beam theory and is mainly caused by the huge bending moment at both ends of the modelled cargo hold section. Whereas the longitudinal failure and the buckling of the side shell are due to the shear force. After the collapse of the bilge structure the side is pushed into the ground. The supporting effect of the ground and the contact area length depend on the ground material.

In particular the double bottom of Ha7p2 has a main orthotropic and inwards buckling field in the middle of the cargo hold between floor fl1 and fl2. It levels with the manholes of the longitudinal girders. The structure loses its contact with the stiff bank due to the inward deformation (see also table 4.9). The deepest buckle in the bottom plating is 0.25 $\mathrm{m}$. The buckling field becomes an outwards fold in the bilge and side structure that continues until stringer s2. At the fold elements are deleted due to high strains. In addition a second but less distinct buckling field appears beside the bulkhead floor b1. One inward longitudinal fold is found in the bilge at the change of the double bottom into the bilge structure with a maximum depth of $0.8 \mathrm{~m}$.
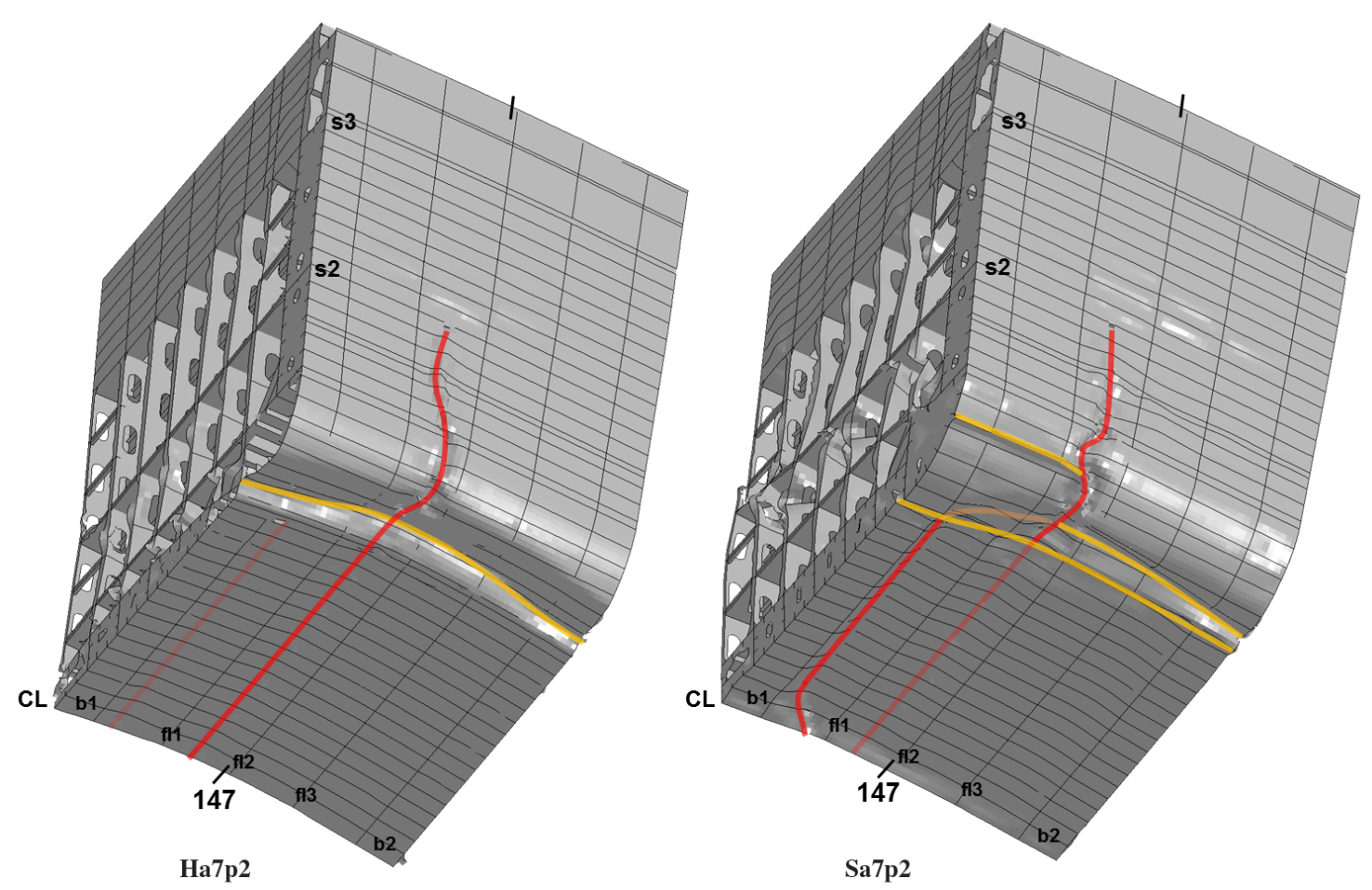

Figure 4.18.: Ha7p2 and Sa7p2: deformation at $5 \mathrm{~m}$ of surface drawdown.

In model Sa7p2 there are one inward transverse fold and buckling field as well as two longitudinal folds. The longitudinal fold is beside the bulkhead structure b1. It has a maximum depth of $0.74 \mathrm{~m}$ compared to the bulkhead floor and is thus $0.5 \mathrm{~m}$ deeper than the buckling field of Ha7p2 at the same position. The outer shell even starts to fracture close to the longitudinal girder at centre line. The longitudinal and orthotropic buckles of Sa7p2 between floor fl1 and fl 2 also level with the manholes of the longitudinal girders 
and are $0.3 \mathrm{~m}$ deeper than those of Ha7p2. At the same longitudinal direction there is the fold of the bilge and side structure that deforms partly inwards and partly outwards. It continues to stringer s 2 and results in fracturing of the outer shell at many positions. The outer shell folds at both sides of the longitudinal girder at the bilge structure, at which point the backmost fold passes through the whole model including the outer shell at both bulkheads. The second longitudinal fold continues from the aft bulkhead b2 through the bilge fold into the longitudinal fold before the fore bulkhead structure b1. It connects the main fold of the double bottom with the fold of the side structure. Furthermore the outer shell of the bilge folds outwards between two longitudinal stiffeners, which underlines the effect that the side structure is pushed into the ground. The collapse of the double bottom is relocated at the indentation of soft ground. Figure 4.19 gives the z-displacement of the sand. The sand is squeezed to the side of the bilge and piles up at the folds. The maximum displacement of $1 \mathrm{~m}$ is found at the bilge radius. The sand piles up to $0.6 \mathrm{~m}$ at the area of the main transverse fold.

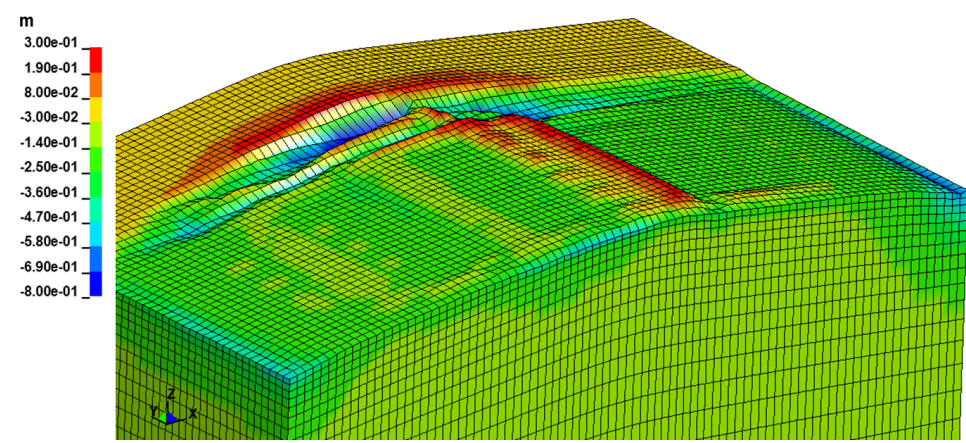

Figure 4.19.: Sa7p2: indentation of sandbank in z-direction at $5 \mathrm{~m}$ of surface drawdown.

Figure 4.20 to 4.22 show the longitudinal stresses of Ha7p2 and Sa7p2 for 1, 3 and $5 \mathrm{~m}$ of tide levels. In the first row of each figure (a) the shell model without the edges is plotted and the scale is fitted to the maximum yield stress of $\pm 355 \mathrm{~N} / \mathrm{mm}^{2}$. The second row (b) shows the outer shell plating and the scale is now fitted to $\pm 315 \mathrm{~N} / \mathrm{mm}^{2}$, which is the highest yield strength of the outer shell. Besides the stresses, the contact length, the faster increasing length to the bow, the failure and the height of the plastic neutral axis are found.

For $1 \mathrm{~m}$ of receding tide the stresses are below the yield stresses. The distribution is similar in both simulations. The stresses of Sa7p2 indicate the longer contact area (see figure 4.20 (b)).

In figure 4.21 the folds and buckling fields of final collapse are already illustrated. The figures show that not only the outer shell of the vessel fails. The above described failure modes are also found in the inner shell plating of the side and bilge structure. The double bottom is lifted up between the bulkheads. The predominant kind of damage is elasto-plastic buckles. Furthermore it becomes clear that the rigid ground has a higher supporting effect onto the double bottom between the bulkheads than the soft ground. Both bulkheads are already in contact with the ground in simulation Ha7p2, whereas in Sa7p2 only the fore bulkhead b2 is in contact. The height of the plastic neutral axis is 
close to stringer s3, comparable to figure 4.12 .
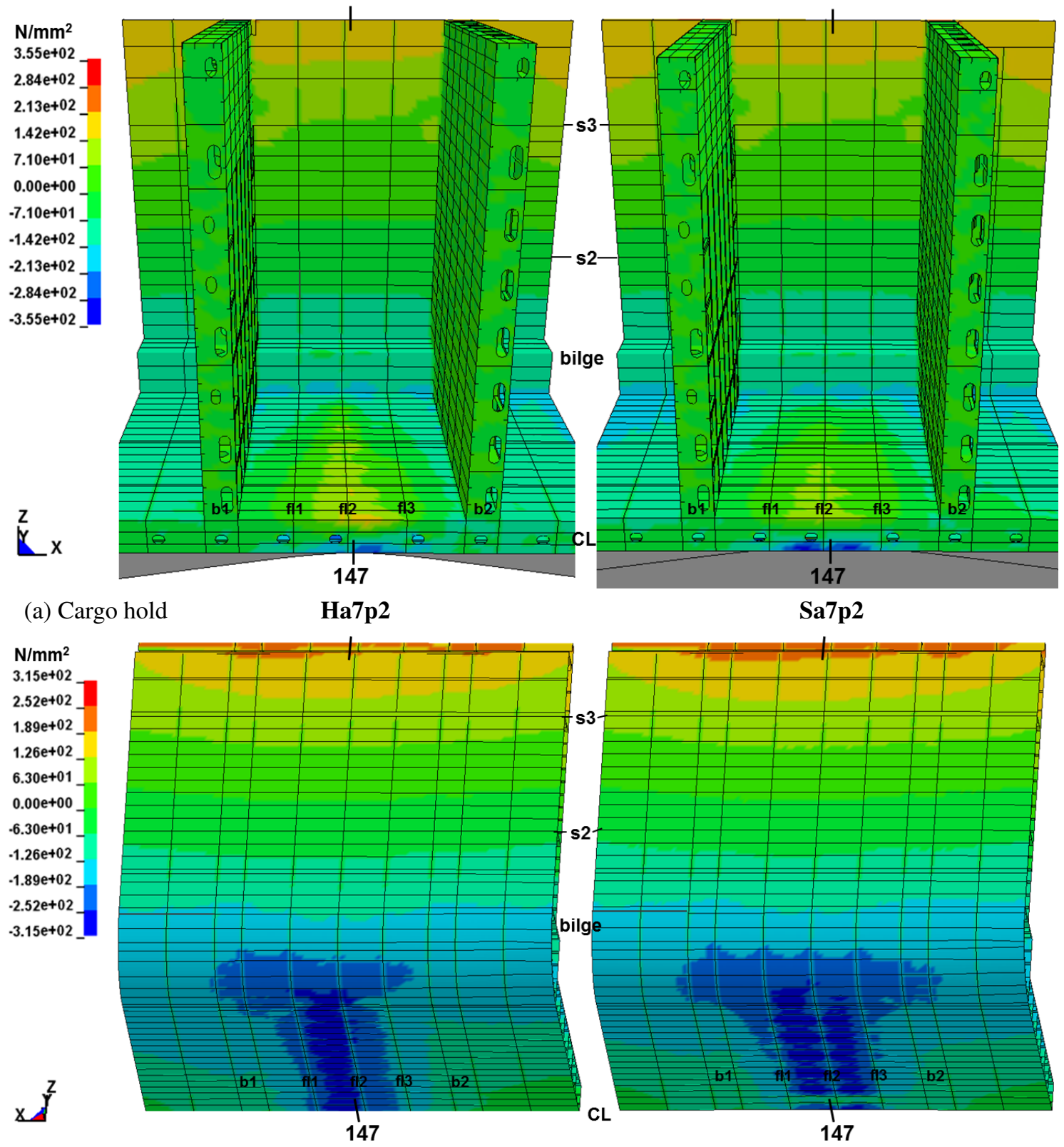

(b) Outer shell

Figure 4.20.: Sa7p2 and Ha7p2: longitudinal stresses for $1 \mathrm{~m}$ of surface drawdown.

If the water level is reduced by $5 \mathrm{~m}$ the modelled double bottom lies on the ground. The hogging bending moment is increased and a significant curvature of the hull can be seen. The curvature of Ha7p2 is smaller than in Sa7p2. A fold in both bulkheads of Sa7p2 can be seen. The folds continue inclined from the stringer of the bilge plating to the height of stringer s2. In the bulkhead plating we again find isotropic buckling as a buckle between each stiffener. The bulkheads of Ha7p2 do not have such a fold and only have a few buckles. Compared to the rigid bank calculation the buckles are spread more 
globally in case Sa7p2. The maximum stresses in both cases are higher than $\pm 500 \mathrm{~N} / \mathrm{mm}^{2}$ in the box girder and in the outer shell at the connection of bilge and double bottom.

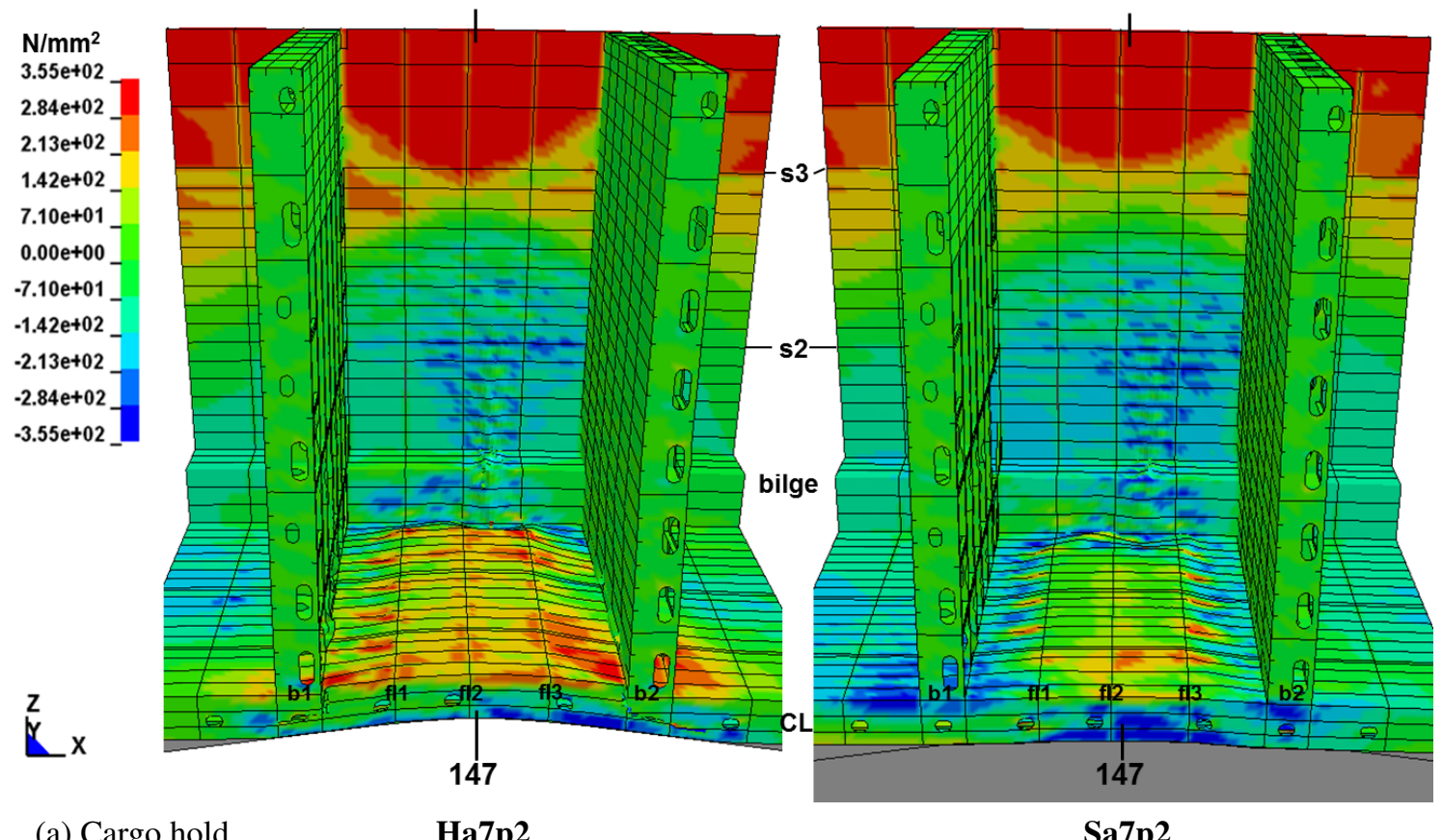

(a) Cargo hold

Ha7p2

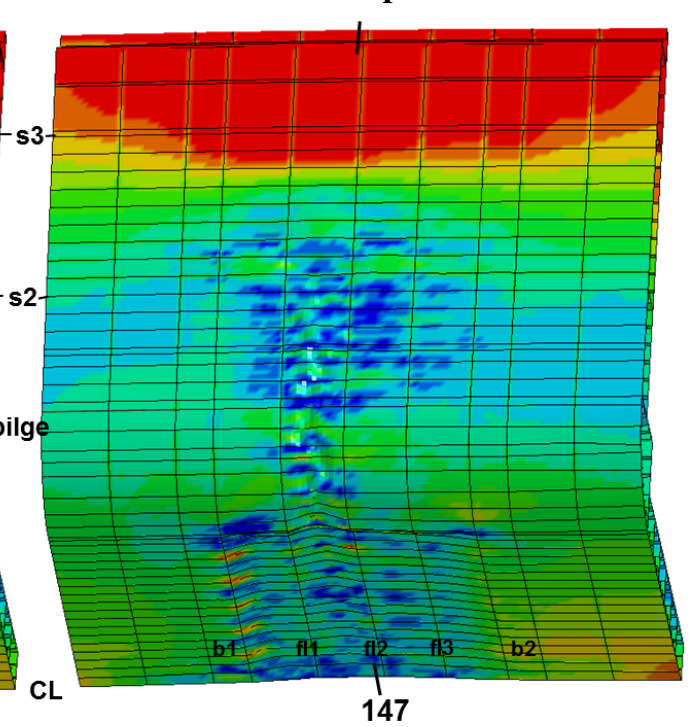

(b) Outer shell

Figure 4.21.: Sa7p2 and Ha7p2: longitudinal stresses for $3 \mathrm{~m}$ of surface drawdown. 

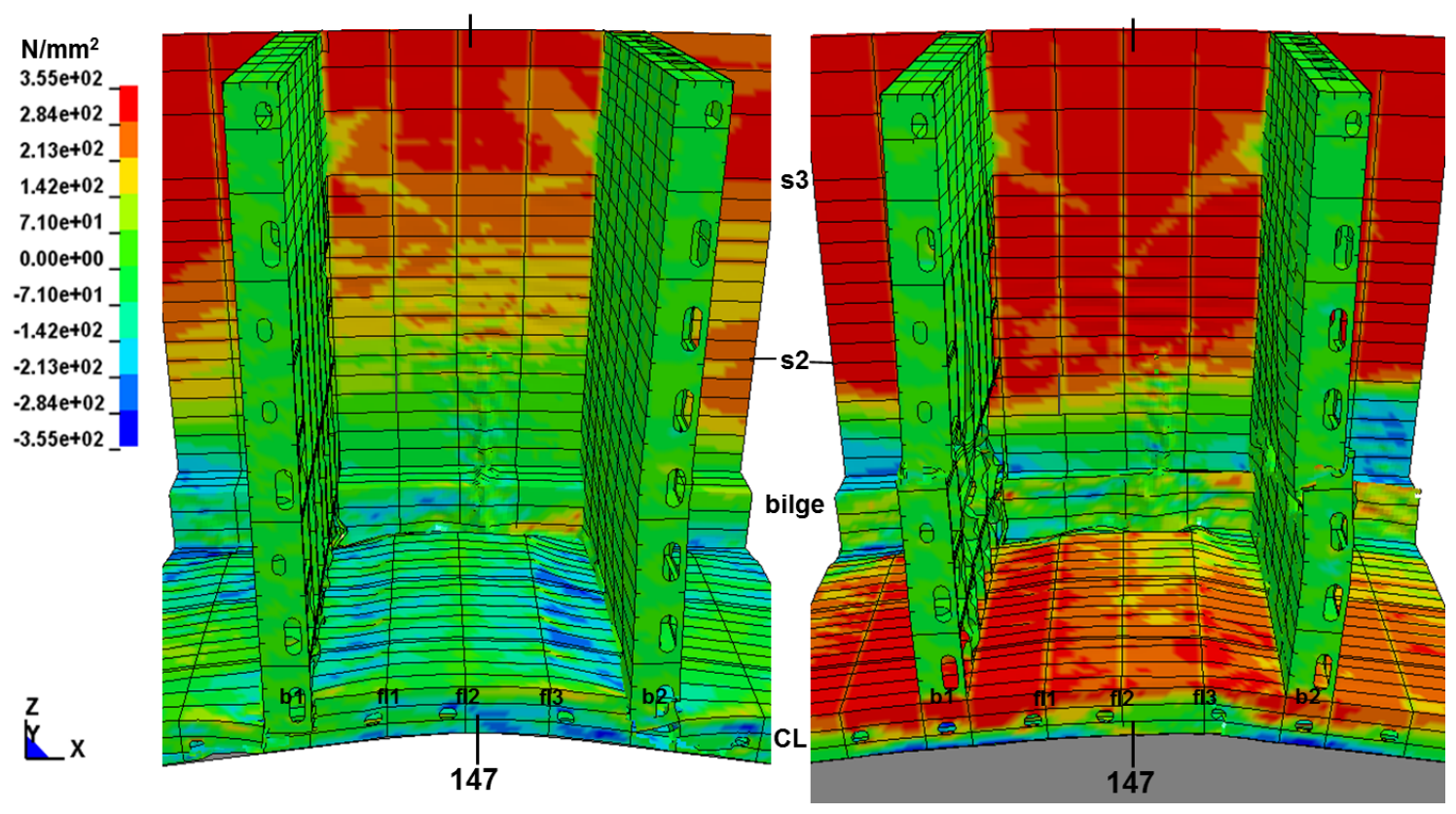

(a) Cargo hold

Ha7p2

Sa7p2

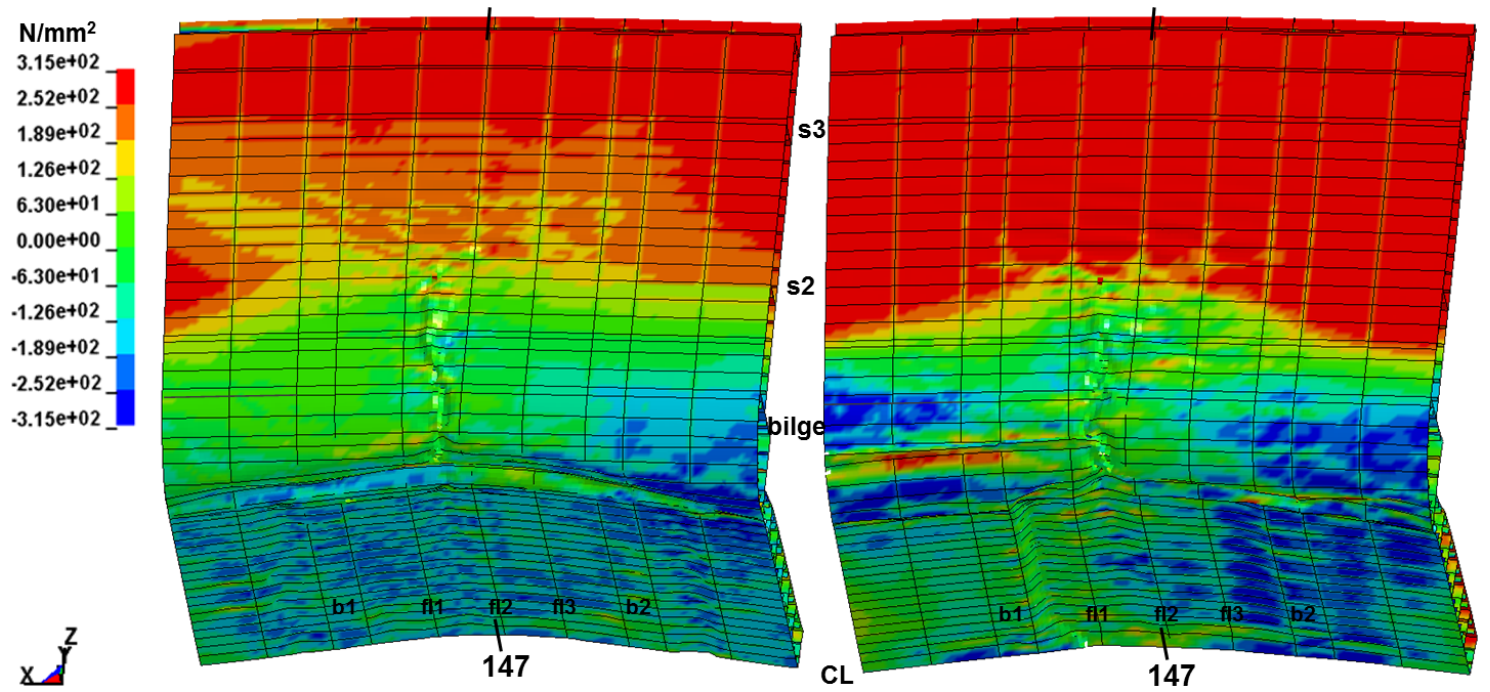

(b) Outer shell

Figure 4.22.: Sa7p2 and Ha7p2: longitudinal stresses for $5 \mathrm{~m}$ of surface drawdown.

\subsubsection{Influence of ground}

\section{Ground parameters}

One calculation is performed with a stiffer sandbank (SGa7p2). The shear modulus is simply considered to be constant over the height of the soft ground. The moment case SGa7p2 is compared to Sa7p2 and Ha7p2 in figure 4.23. 


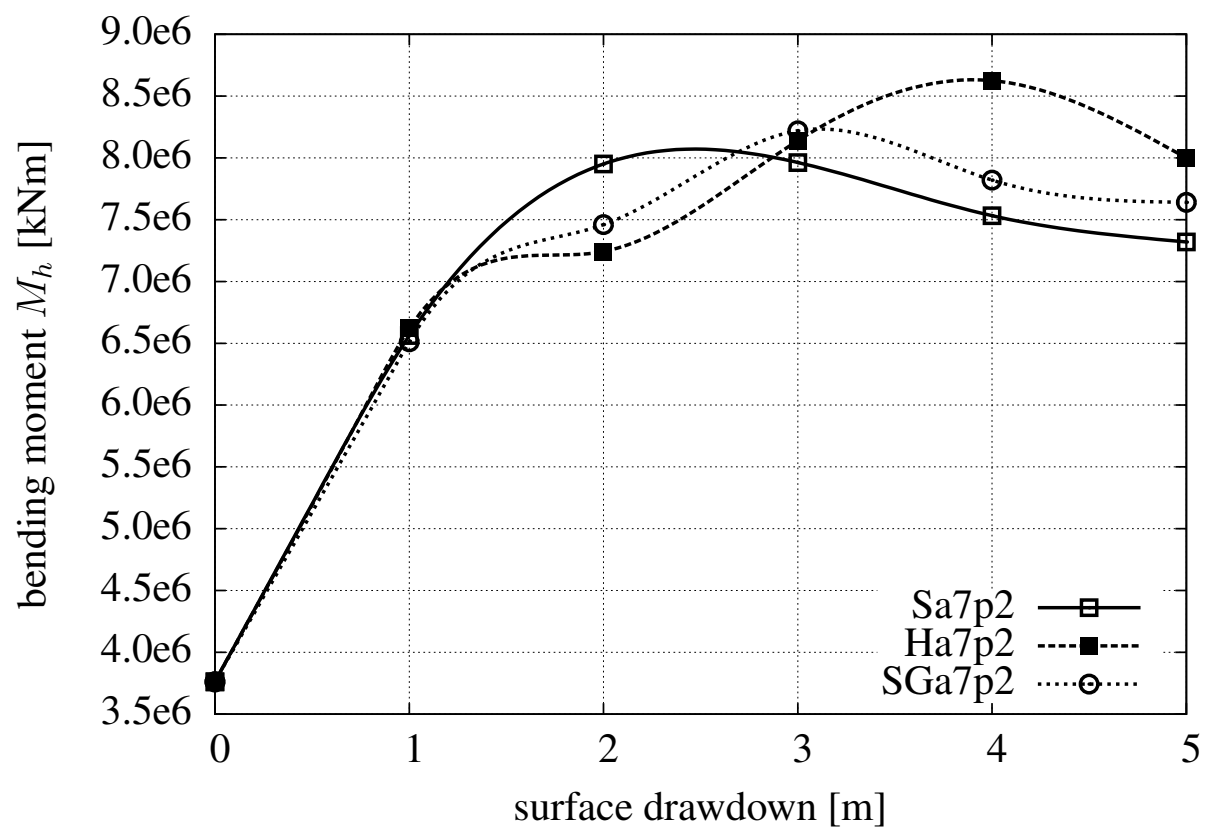

Figure 4.23.: Sa7p2, Ha7p2 and SGa7p2: bending moment $M_{h}$ at $147 \mathrm{~m}$ AP.

The curve of SGa7p2 is qualitatively equal to Ha7p2 for 0 to $3 \mathrm{~m}$ of receding tide. The ultimate strength of SGa7p2 lies between Sa7p2 and Ha7p2 and is reached with $3 \mathrm{~m}$ of water level reduction.

The collapse of SGa7p2 is shown in the appendix in figure B.7. It resembles Sa7p2: one transverse fold in the double bottom next to bulkhead b1, one buckling field between floor fl1 and fl 2 and one transverse fold. Only the fold of the side structure is between the web girder fl 1 and fl 2 and the second longitudinal fold is continuous on the other side of the transverse girder in the bilge plating. The maximum displacement of SGa7p2 in $\mathrm{z}$-direction at $147 \mathrm{~m} \mathrm{AP}$ is $0.23 \mathrm{~m}$ less than that of $\mathbf{S a 7 p 2}$.

\section{Ground geometry}

Three other ground geometries are calculated as given in table 4.2. Their moments are given in figure 4.24 together with Sa7p2. Their final collapse modes appear in appendix B.5.2. In all cases the ultimate strength is reached with $3 \mathrm{~m}$ of receding tide. The bigger the initial contact area or the smaller the slope angle the higher the maximum moments of cross section $147 \mathrm{~m}$ AP.

Sa15p2 has the lowest ultimate hull girder strength and the most severe failure. In this case the double bottom fractures over its complete breadth between floor fll and the bulkhead floor b1 (see figure B.8) so that the moment falls below the value of $1 \mathrm{~m}$ of water level reduction at the end of the simulation.

The curves of simulation Sa7p22 and Sa7p22w10 are qualitatively the same. The smaller breadth of the sandbank results in a $10 \%$ smaller ultimate strength. The final collapse mode of both simulations is different. In Sa7p22 there is one transverse fold that is lo- 


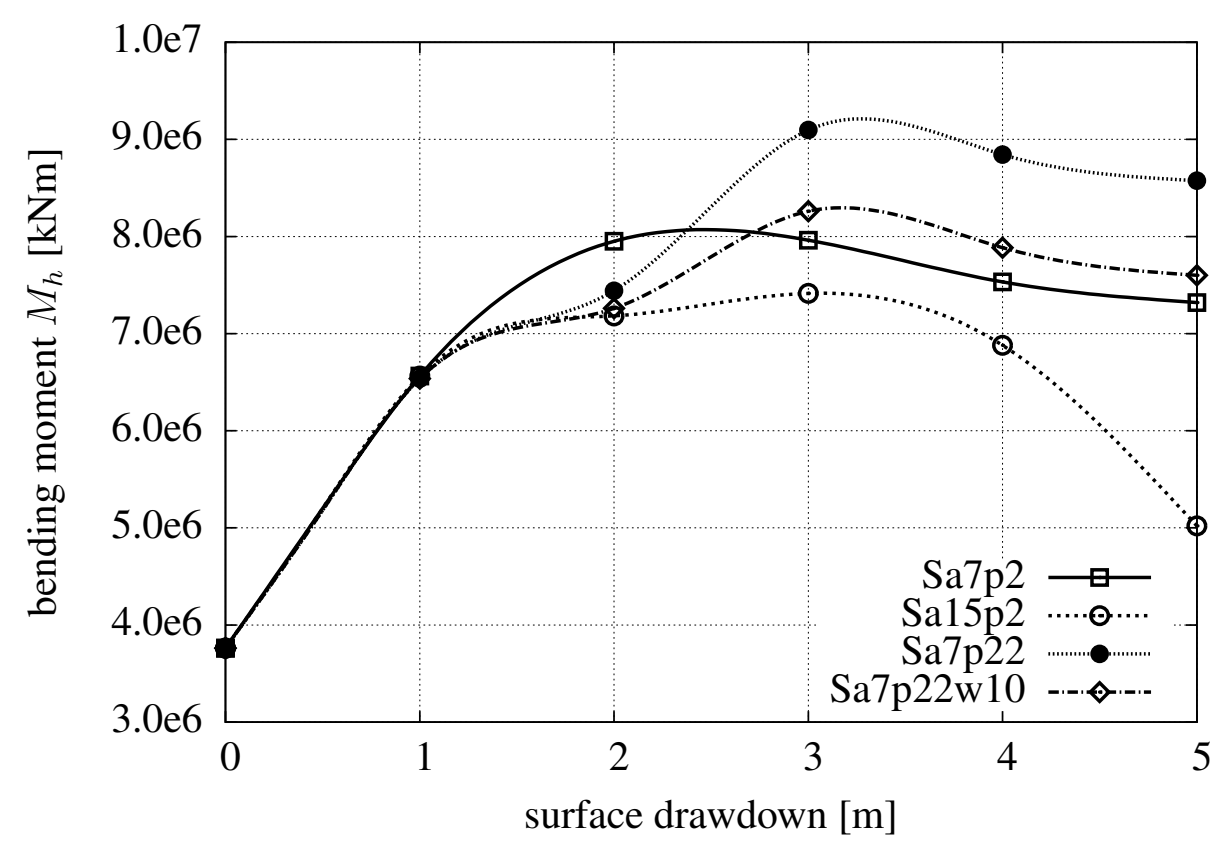

Figure 4.24.: Sa7p2, Sa15p2, Sa7p22 and Sa7p22w10: bending moment $M_{h}$ at $147 \mathrm{~m} \mathrm{AP.}$

cated in front of bulkhead b1 and continues from the centre line to the bilge into the side structure until stringer s2. On the other side of the cargo hold beside bulkhead b2 there is a single fold in the bilge structure (see figure B.9). The longitudinal fold is again beside the longitudinal girder close to the bilge. In contrast, in case Sa7p22w10 two continuous longitudinal folds are found on each side of the longitudinal girder which is the closest to the the sandbank edge (refer to figure B.10). Furthermore, Sa7p22w10 has only one transverse fold from the longitudinal girder just mentioned until stringer s2.

\subsection{Analysing the Fowairet stranding incident}

In the introduction (chapter 1) the stranding incident of the container vessel Fowairet is already described. This incident is compared to the above introduced method to analyse its applicability to real scenarios. The listed information had to be reconstructed or assumed with the help of photographs or the internet:

- ship: main dimensions, construction, load case, changed buoyancy

- ground: geometry, parameters, position relative to ship, tidal range

The following subsection reveals that the vessel Postpanmax, for which all information is known, is comparable to the Fowairet. Modern container vessels have a similar construction, especially in the midship section. 


\subsubsection{Specifics of the Fowairet}

The dimensions the Fowairet are given in table 4.11 and compared to the Postpanmax.

After the accident the fractured midship section was removed. Figure 4.25 shows the sliced Fowairet in the dock before the new midship section is floated into position. The photograph is overlaid with a suitable construction drawing of a main frame. When compared to the main frame construction drawing of the Postpanmax in figure 4.3 it is clear that they are similar. The differences result from their different height. Examining the outer shell damage at the starboard side and the bottom shell damage of the Fowairet, given in the first row of figures 4.26 and 4.27 , one can identify missing construction details.

The Fowairet was on its way to the Port of Antwerp, probably travelling with load case arrival. The draught before the incident was reconstructed to be equivalent to the draught for the arrival load case of the Postpanmax.

Table 4.11.: Dimensions of the Fowairet and the Postpanmax.

\begin{tabular}{lrr}
\hline & Fowairet & Postpanmax \\
\hline length $L_{o a}[\mathrm{~m}]$ & 276.50 & 294.10 \\
length $L_{p p}[\mathrm{~m}]$ & 259.90 & 285.60 \\
breadth $B[\mathrm{~m}]$ & 32.20 & 40.00 \\
height $D[\mathrm{~m}]$ & 21.20 & 24.20 \\
scant. draught $T_{\text {scant }}[\mathrm{m}]$ & 12.50 & 13.50 \\
speed $v[\mathrm{kN}]$ & 24.00 & 24.00 \\
\hline
\end{tabular}

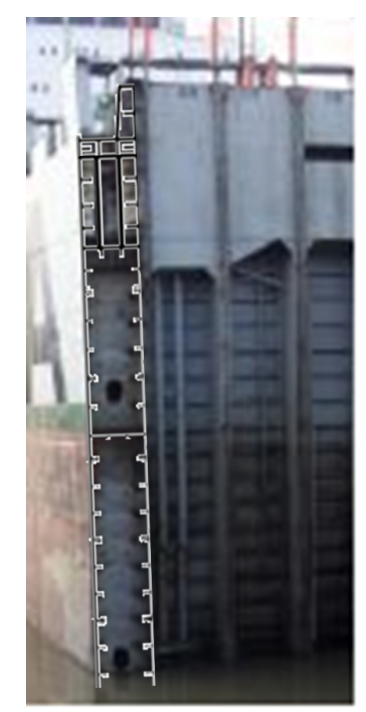

Figure 4.25.: Main frame of the Fowairet.

\subsubsection{Specifics of the incident}

Loaded with 1952 TEU, the Fowairet grounded in the Western Scheldt close to Walsoorden vis-á-vie to Hansweert one hour after high tide. The observed spring tidal range 
at Hansweert is $5 \mathrm{~m}$ and in Antwerp as high as $5.85 \mathrm{~m}$ (see van Rijn [78]). The salvage operation took two days. During the third flood tide after the accident the vessel was refloated.

The river bed of the Scheldt consists of sand (see among others European Union [16]). The vessel stranded at the midship section. The photographs show that the plateau must have been short compared to the ship's length.

\subsubsection{Comparing the damage of the Fowairet to Sa7p2}

The Fowairet stranding incident is compared to Sa7p2. As described above, most assumptions made for $\mathbf{S a 7 p 2}$ are similar to the accident.

In figures 4.26 and 4.27 the damage to the Fowairet (the first row) is compared to the damage of Sa7p2 after $5 \mathrm{~m}$ of surface drawdown (second row). The damage pictures of Sa7p2 have the same colours as Fowairet. The construction below the shell is given with red lines. The numbers refer to the constructional elements, already given in table 4.10. The spacing of web frames/floor, (bottom) longitudinal frames and the bulkhead length can be identified from the photograph and are given in table 4.12. The cargo hold length between two bulkheads is identical for both vessels due to normed container sizes. The spacing between the aft (left) bulkhead and the next two web frames is $3250 \mathrm{~mm}$ each for Postpanmax. The following two frame spacings are $3050 \mathrm{~mm}$ long.

Table 4.12.: Spacing of the Fowairet and the Postpanmax.

\begin{tabular}{lrrr}
\hline & number & Fowairet & Postpanmax \\
\hline web frame/floor spacing [mm] & 2 & 3150 & $3250 / 3050$ \\
longitudinal frame spacing [mm] & 3 & 860 & 970 \\
cargo hold length [m] & - & 126000 & 126000 \\
bulkhead length [mm] & 1 & 1500 & 1850 \\
\hline
\end{tabular}

The side pictures of the Fowairet and Sa7p2 show similar buckles of the outer shell. Both show the isotropic buckling and the main fold. The Fowairet folds directly at the middle web frame of the cargo hold and Sa7p2 has its fold between web frames (fl1 and fl2). The fold of Sa7p2 has the same distance from the aft bulkhead as the fold of the Fowairet due to the different frame spacing. In addition, both deep folds end at the stringer (4/s2) and have at their ends an immense deformation of the outer shell.

The main fold of the bottom shell plating can be identified very well in both damage pictures of figure 4.27. The outer shell plasticises and deforms inward. The fold is close to the bulkhead floor and extends with a slope into the bilge plating by crossing one floor. The bilge structure has in both cases the most severe failure. The bilge plating of Fowairet totally plasticises (see right corner of damage picture). It is known from accident reports that the shell fractured. Although it is not visible at the picture the shell plating of the Fowairet probably fractured in the area of the bilge because in the bilge plating of Sa7p2 elements are deleted. Furthermore, in both cases there are orthotropic buckles between the longitudinal girders. 

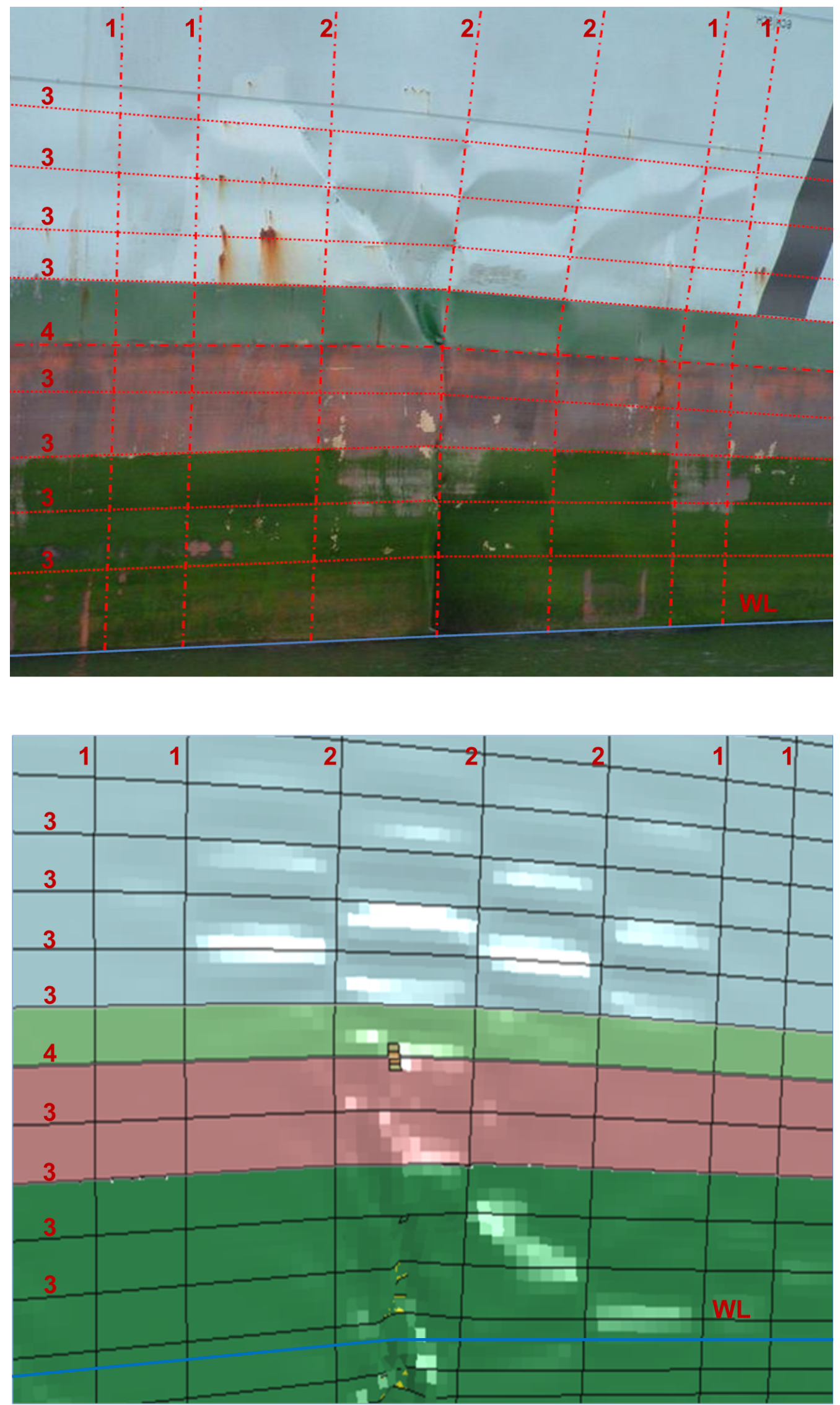

Figure 4.26.: Fowairet vs. Sa7p2: deformation of starboard outer shell. 

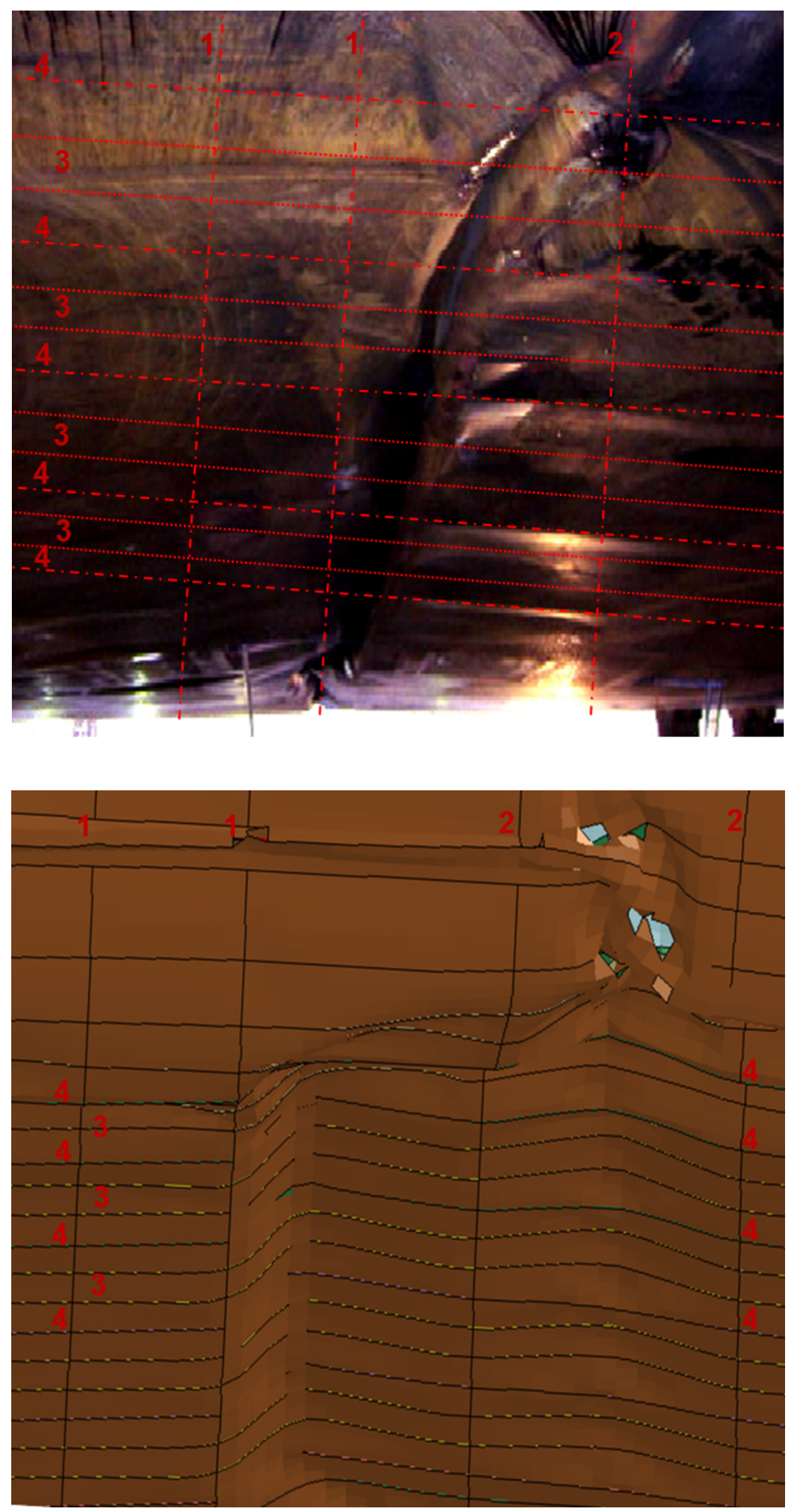

Figure 4.27.: Fowairet vs. Sa7p2: deformation of bottom shell. 
Figure 4.28 shows the Fowairet during the incident with its overall bending. The vessel is still stranded on the sandbank and the structure has already failed. On the right hand side the FE model from simulation Sa7p2 is overlaid to show the similarities of the hull bending in the damaged section.
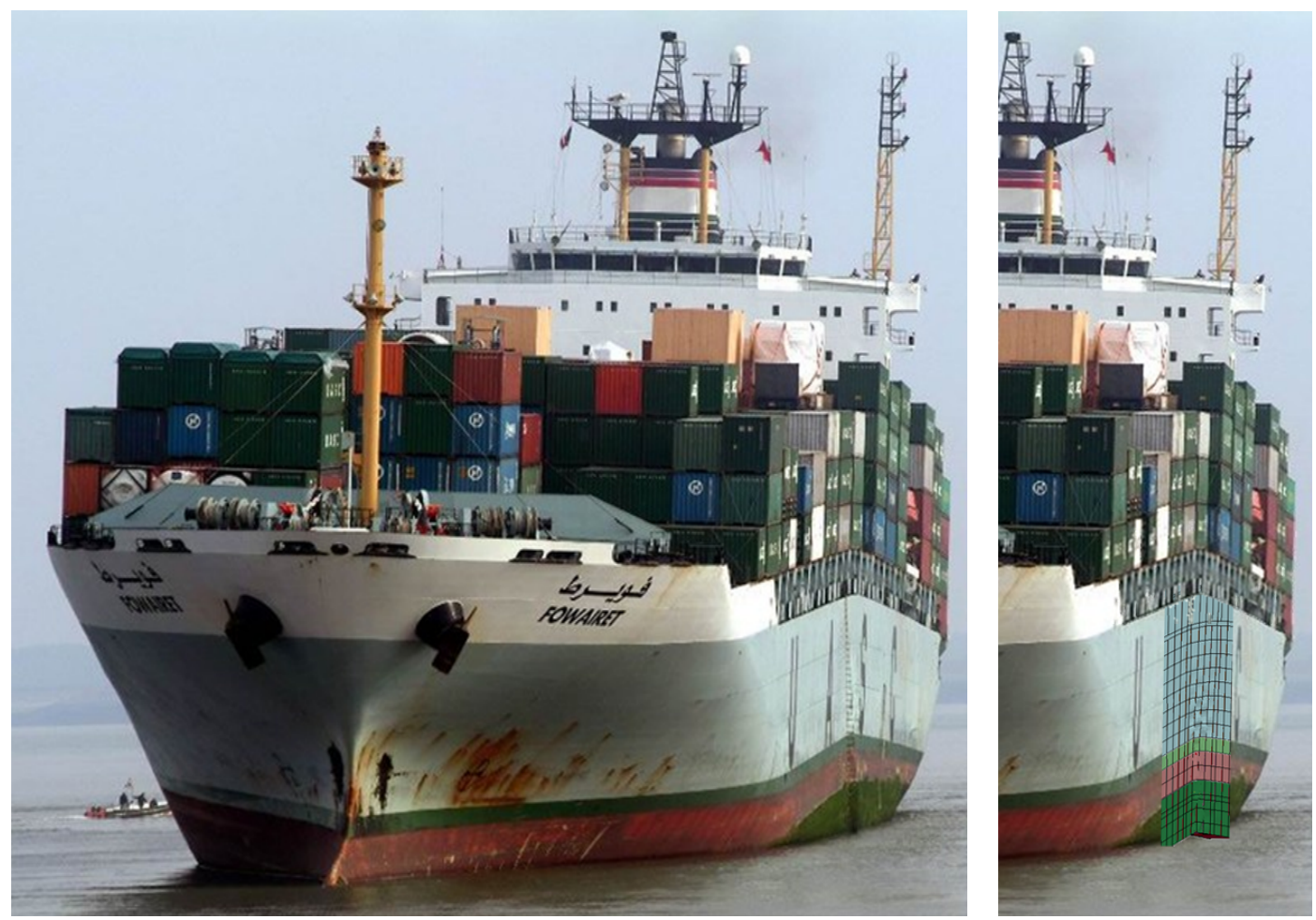

Figure 4.28.: Fowairet vs. Sa7p2: bending of hull.

The FE simulation Sa7p2 reproduces the damage to the container vessel Fowairet. The other results of Sa7p2, described above, should be valid for the incident of Fowairet.

\subsection{Results of the bow and the stern scenarios}

The results for stranding in the bow $(250 \mathrm{~m} \mathrm{AP})$ and the stern region $(65 \mathrm{~m} \mathrm{AP})$ are again described for geometry a $7 \mathrm{p} 2$.

\subsubsection{Stranding at the bow section}

The damage to the structure is mainly attributable to the indentation of the ground, namely the shear forces. In contrast to stranding in the midship section the external bending moments at the edges of the modelled cargo hold section are very small. The bending moment at $250 \mathrm{~m}$ AP is more than 10 times smaller than for the midship scenario. Furthermore the bow section is a relatively stiff section.

The curves of the bending moment at $250 \mathrm{~m} \mathrm{AP}$ and the shear forces at 239 and $261 \mathrm{~m} \mathrm{AP,}$ 
are given in figures 4.29 and 4.30 together with the results of case A1. The cross section at 239 and $261 \mathrm{~m}$ AP lie beside the bulkheads (see figure 4.31).

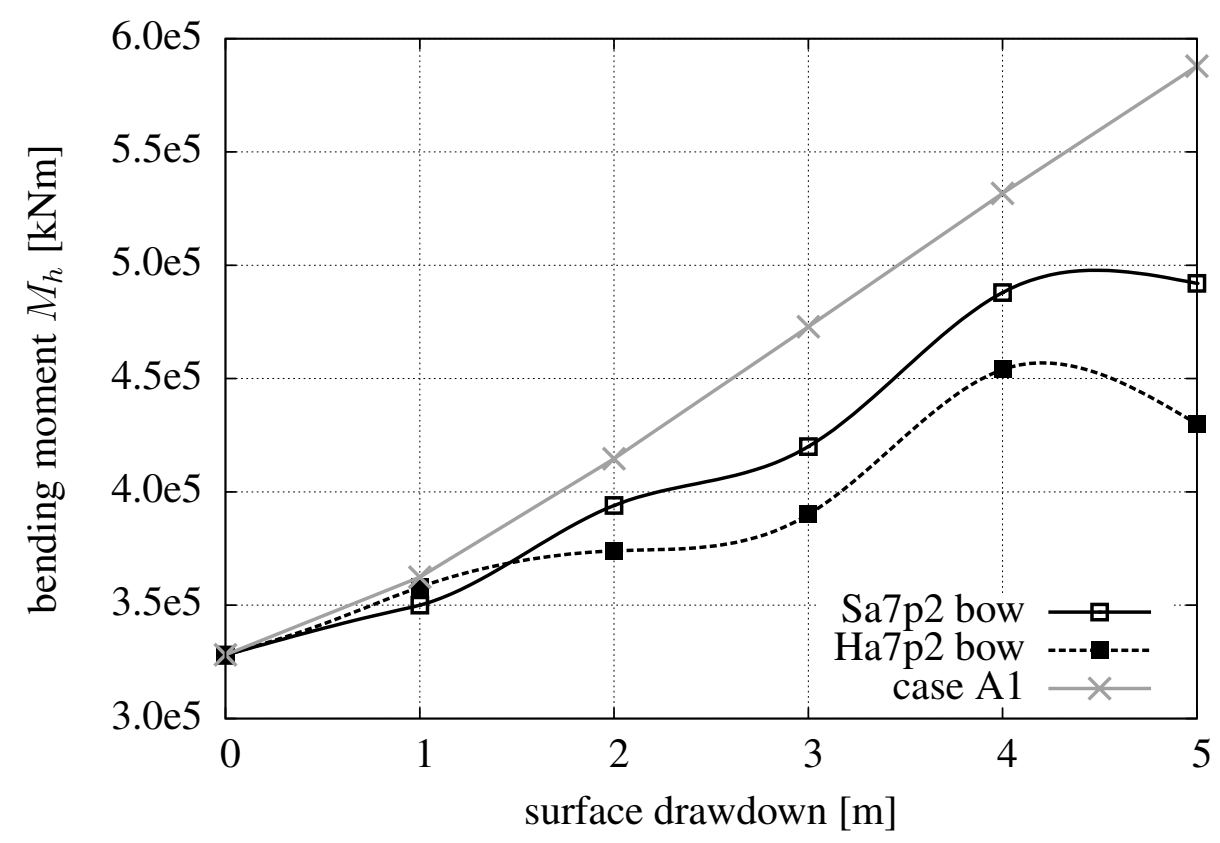

Figure 4.29.: Sa7p2 bow and Ha7p2 bow: bending moment $M_{h}$ at $250 \mathrm{~m} \mathrm{AP.}$

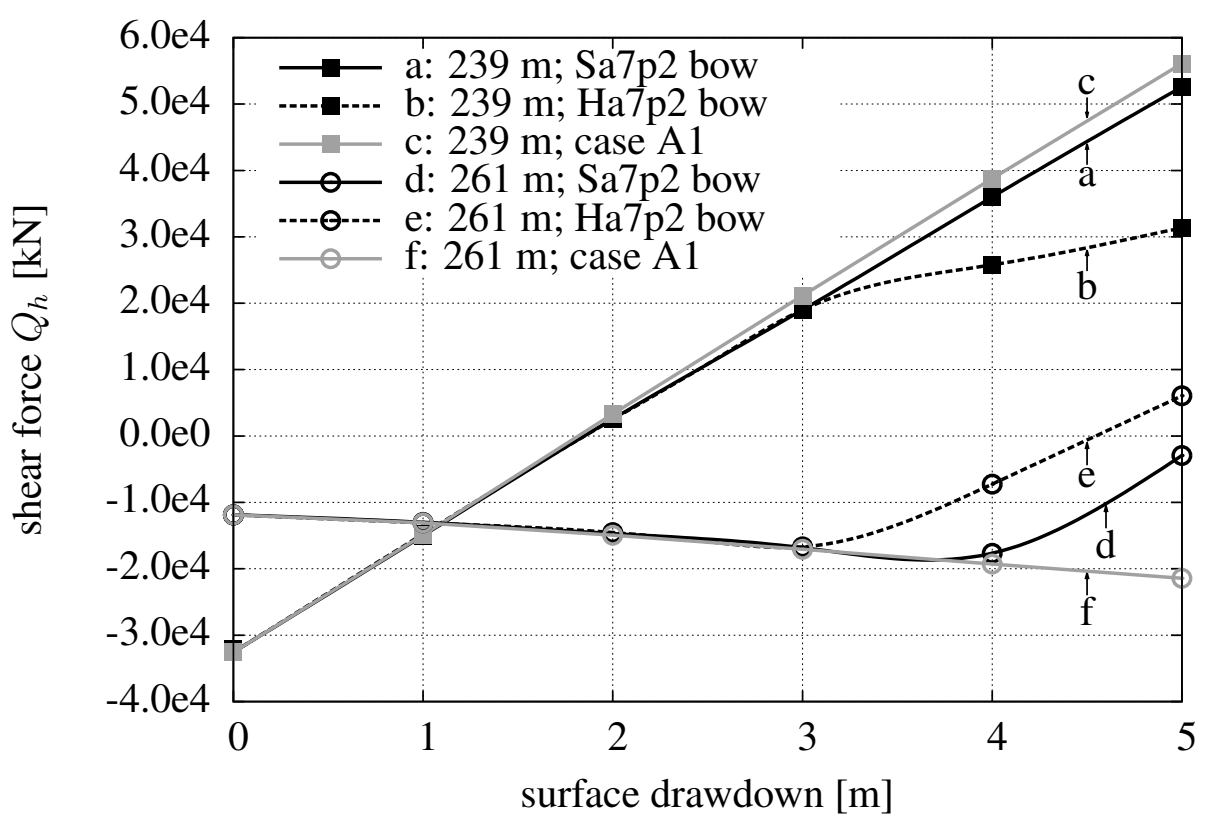

Figure 4.30.: Sa7p2 bow and Ha7p2 bow: shear forces $Q_{h}$ at 239 and $261 \mathrm{~m} \mathrm{AP.}$

The moment curves for Ha7p2 bow and Sa7p2 bow are comparable. Sa7p2 bow can bear higher moments and forces and therefore the hard ground is the conservative 
estimation. In simulation Ha7p2 bow the curve declines after $4 \mathrm{~m}$ of receding tide. The moments at $1 \mathrm{~m}$ of surface drawdown differ again due to different contact lengths. Up to $3.8 \mathrm{~m}$ of receding water Sa7p2 bow has the longer contact area length than Ha7p2 bow and from $3.8 \mathrm{~m}$ onward this reverses. The effect is reflected by the shear forces (see figure 4.30). If the water level is reduced by $5 \mathrm{~m}$ the contact lengths are: $33 \mathrm{~m}$ for case $\mathbf{S a 7} \mathbf{p} 2$ bow and $35.5 \mathrm{~m}$ for case Ha7p2 bow.

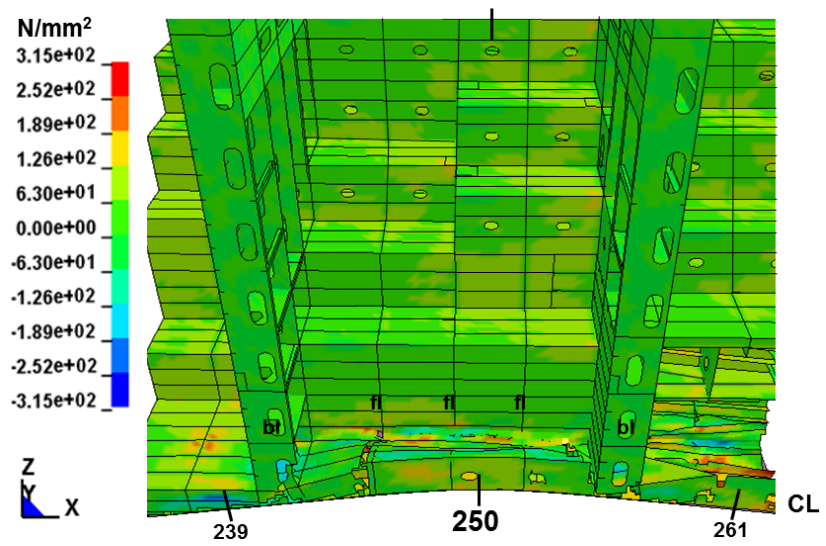

(a) Cargo hold

Ha7p2

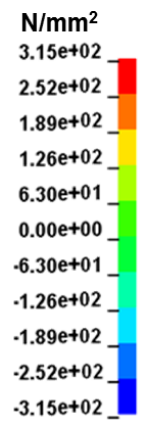

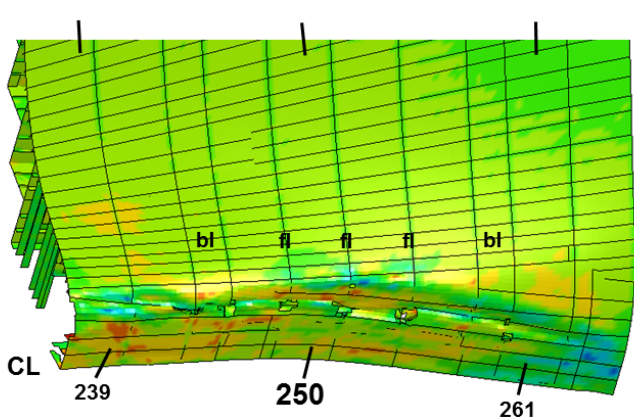

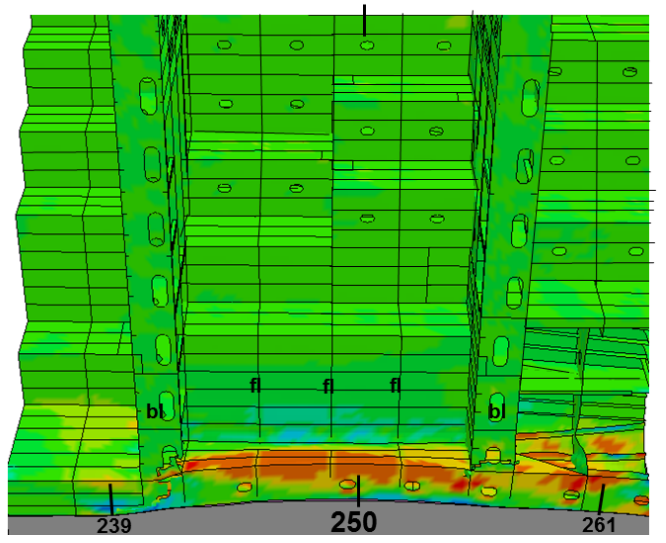

Sa7p2

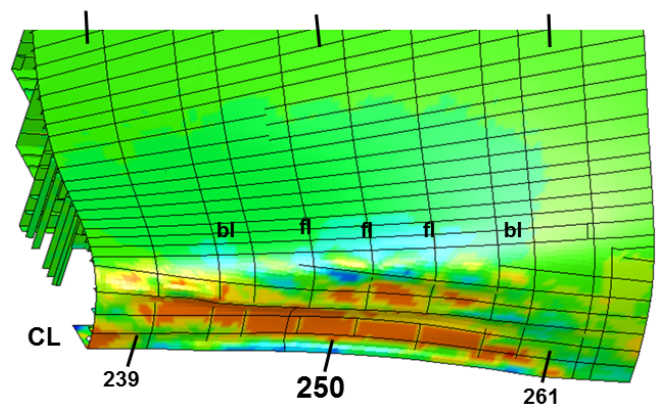

(b) Outer shell

Figure 4.31.: Sa7p2 bow and Ha7p2 bow: longitudinal stresses for $5 \mathrm{~m}$ of surface drawdown.

The first elements fail at $1.5 \mathrm{~m}$ for Ha7p2 bow and the yield stresses are reached at 2 $\mathrm{m}$ of water level reduction. For Sa7p2 bow the elements start to fail and the yield stresses are not exceeded until the receding tide reaches $2.9 \mathrm{~m}$. Only internal structural elements of Sa7p2 bow fail, whereas the outer shell of Ha7p2 bow already ruptures at $3.1 \mathrm{~m}$ of receding tide.

In both simulations high stresses and strains are found in the double bottom only at the contact (see figure 4.31). In case Sa7p2 bow the high stresses are spread wider. But most parts of the model have stresses below the yield strength and an influence of the external bending moment cannot be found.

The figures of the final collapse given in appendix B.6.1 show again that the damage to Ha7p2 bow is more severe than to that Sa7p2 bow. In case Ha7p2 bow there is one deep longitudinal fold through the model around the longitudinal stiffener of the bilge that is 
directly at the transition from vertical to horizontal plating. At the fold the outer plating fractures. In Sa7p2 bow the damage is spread more globally. There are three shorter longitudinal folds in the area of the bilge. The deepest and longest one develops at the same position as for Ha7p2 bow.

The bending mode of the complete vessel changes from hogging into sagging because the vessel trims to the stern and produces a buoyancy over-plus at the stern.

The different ground geometries have the same influence on the ultimate hull girder strength as for the midship scenario. The vessel survives the stranding scenario Sa7p22 bow without any damage and reaches the moments and shear forces forecast by the beam theory, whereby case Sa15p2 bow can sustain the smallest forces and moments and it has the most severe damage out of the soft ground variation. The double bottom tears open directly at the fore bulkhead and the three transverse folds described for Sa7p2 bow deepen. The final collapse of Sa15p2 bow is also given in appendix B.6.1.

Further details concerning the damage mode and the damage description of each case are documented in Steimer [76].

\subsubsection{Stranding at the stern section}

If the vessel strands at $65 \mathrm{~m}$ AP the structure does not sustain severe damage. The small deformations of the structure are caused by the indentation of the ground. Again the external bending moments at the edges of the modelled cargo hold section are very small and the aft body construction is the stiffest section of the vessel. In addition, the stern section can bear high shear forces due to its closed rooms in the machinery room.

The shear forces at cross sections 58 and $74 \mathrm{~m} \mathrm{AP}$ are somewhat smaller than for case A1 corresponding to the different contact length and are given in figure 4.33. Figure 4.32 shows the moments Sa7p2 stern, Ha7p2 stern and case A1 at cross section $65 \mathrm{~m}$ AP. Ha7p2 stern reaches its ultimate strength for $4 \mathrm{~m}$ of receding tide, whereas Sa7p2 stern could bear even higher forces up to a tidal range of $5 \mathrm{~m}$.

The longitudinal stress distribution for a surface drawdown of $5 \mathrm{~m}$ in figure 4.34 reflects the different capacities. Sa7p2 stern reaches only at the contact area stresses higher than the yield stresses. In Ha7p2 stern the high stresses and strains are found in more parts of the vessel. Via the stresses at the coaming in both cases a minimal influence of the bending moment is recognised. Only in calculation Ha7p2 stern some elements of the internal structure are deleted.

The figures of the final collapse given in appendix B.6.2 show that the damage to Sa7p2 stern is insignificant. In case Ha7p2 stern there is one main longitudinal isotropic buckling field through the model. It is located at the longitudinal bulkhead to which the brackets are connected. The brackets also buckle (see figure 4.34 (a)).

The different ground geometries have a very small influence on the ultimate hull girder strength. The vessel survives the stranding scenario Sa7p22 stern without any damage and even in case Sa15p2 stern the final damage is less than Ha7p2 stern (given additionally in appendix B.6.2). 


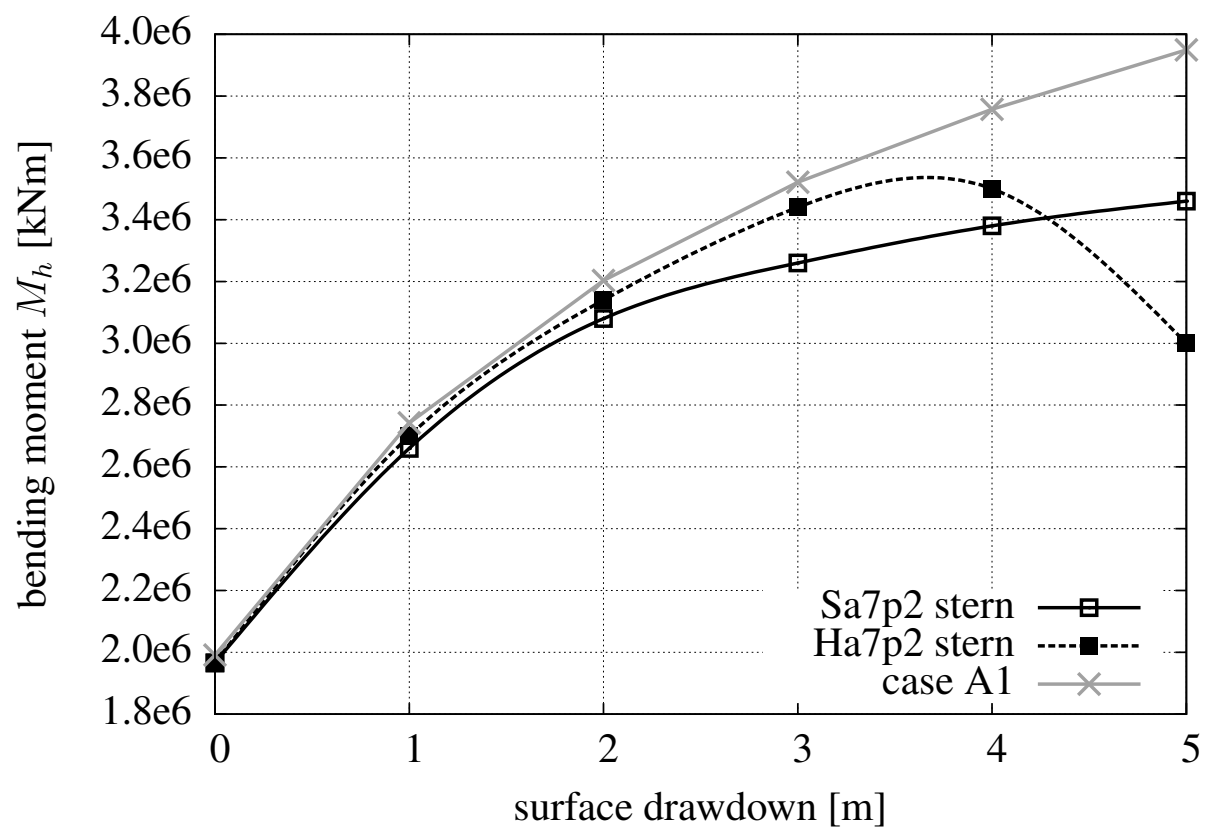

Figure 4.32.: Sa7p2 stern and Ha7p2 stern: bending moment $M_{h}$ at $65 \mathrm{~m} \mathrm{AP.}$

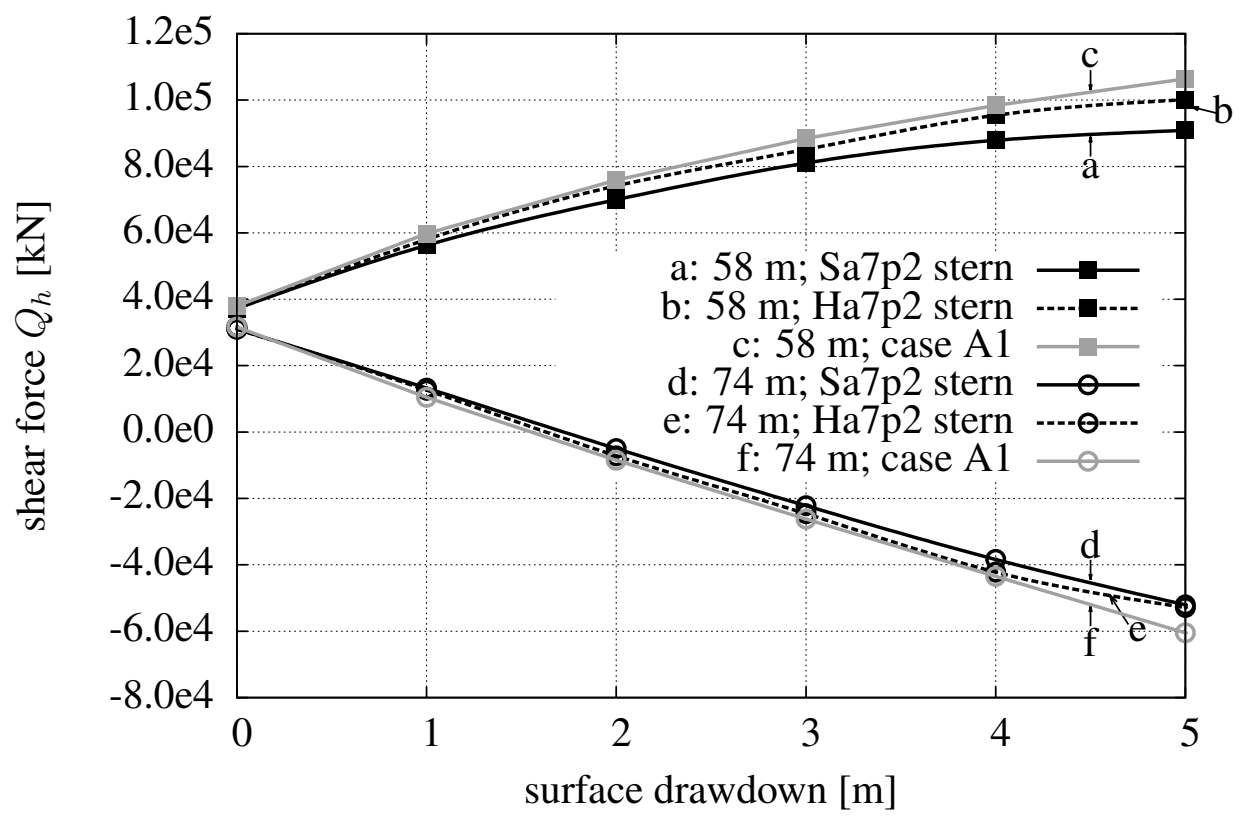

Figure 4.33.: Sa7p2 stern and Ha7p2 stern: shear forces $Q_{h}$ at 58 and $74 \mathrm{~m} \mathrm{AP.}$

A detailed discussion of stranding at $65 \mathrm{~m} \mathrm{AP}$ can be found in Meier [42]. 

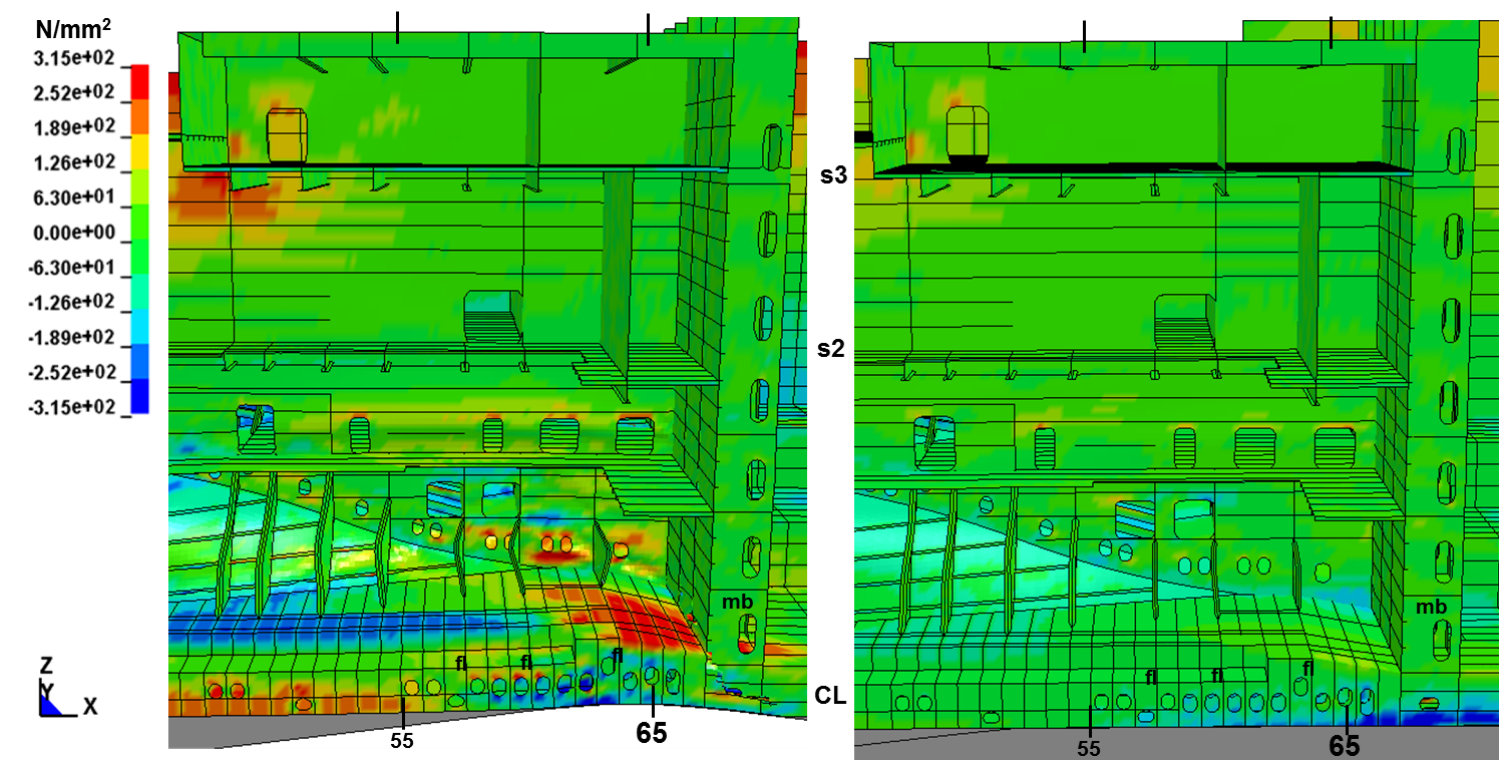

(a) Cargo hold

Ha7p2

Sa7p2

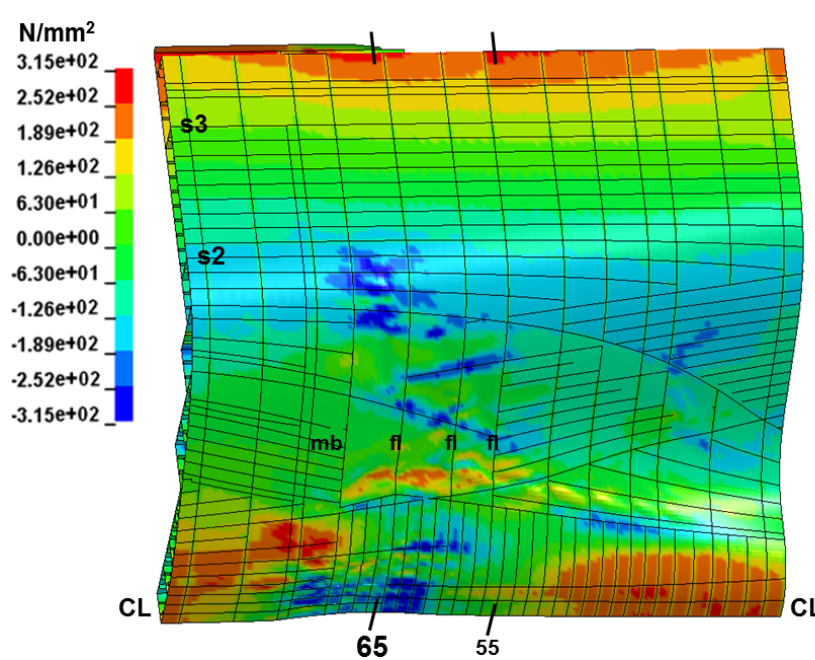

(b) Outer shell

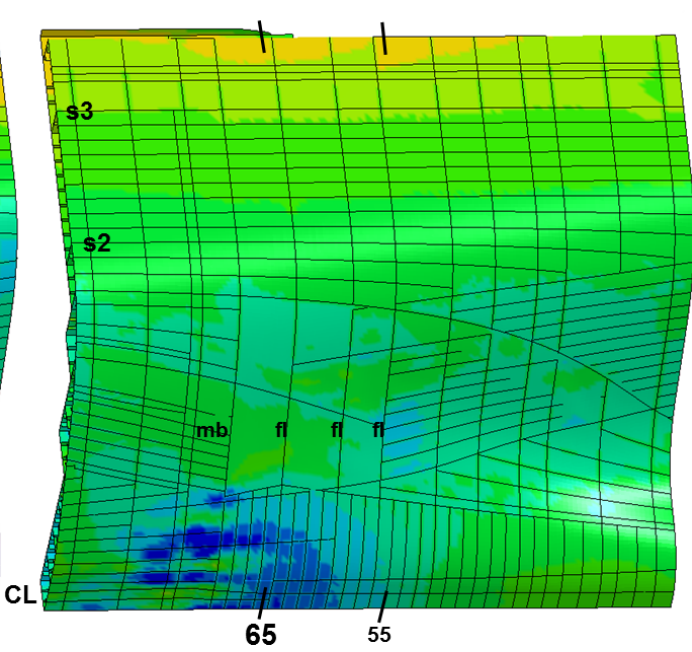

Figure 4.34.: Sa7p2 stern and Ha7p2 stern: longitudinal stresses for $5 \mathrm{~m}$ of surface drawdown.

\subsection{Relation of moment and shear force in the midship, bow and stern scenarios}

The relation of moment to shear forces is compared for all six a7p2-cases (Ha7p2 stern, Sa7p2 stern, Ha7p2, Sa7p2, Ha7p2 bow and Sa7p2 bow) according to section 4.5.1. The bending moments and shear force jumps for $5 \mathrm{~m}$ of surface drawdown are scaled by those of case A1, e.g. for case Sa7p2: $M_{S a 7 p 2} / M_{\text {case } A 1}$ at $147 \mathrm{~m} \mathrm{AP}$ as already given in figure 4.15 .

Figure 4.35 gives one point for each of the six cases. The normalised moments are plot- 
ted over the normalised shear force jumps. The figure shows that the ability to absorb both external moment and shear force influences the degree of damage. The smaller the normalised moment and shear force the greater is the degree of damage. The stern scenario shows the least damage followed by the bow and the midship scenario (stern $<$ bow $<$ midship). The degree of damage cannot be concluded from the height of the external forces and moments. Ordering the scenarios by the height of the forces/moment results in bow $<$ stern $<$ midship. When stranding amidships the structure has to sustain by far the highest moments and shear forces.

The moments and shear forces are related. But the ability to absorb the moment is decisive for the degree of damage. The points of the more severe cases Ha7p2 stern, Sa7p2 and Ha7p2 bow reach smaller $M_{a 7 p 2} / M_{\text {case } A 1}$ and higher $Q_{S a 7 p 2} / Q_{\text {case } A 1}$ than the cases with less damage.

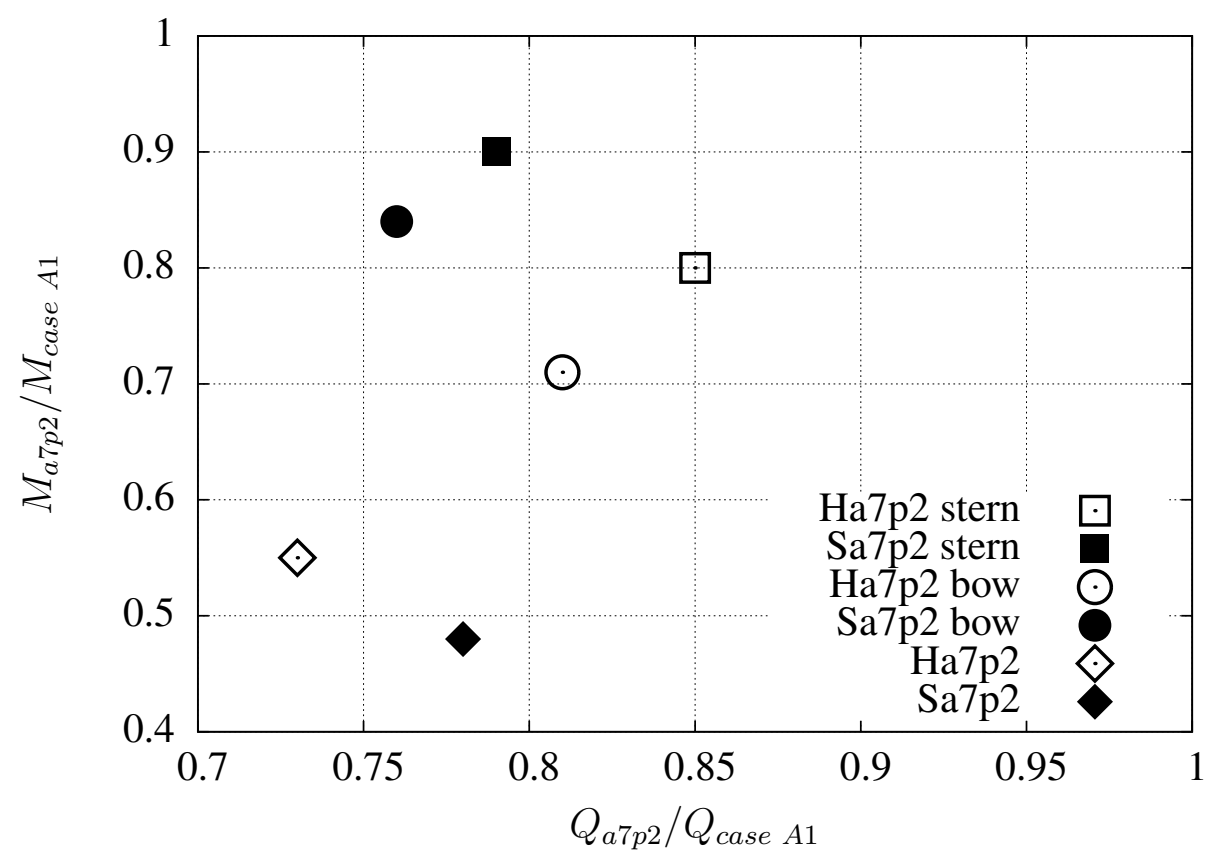

Figure 4.35.: Ha7p2 stern, Sa7p2 stern, Ha7p2, Sa7p2, Ha7p2 bow and Sa7p2 bow: normalised moments and shear forces at $147 \mathrm{~m}$ AP.

\subsection{Discussion}

The midship stranding scenario is the worst-case scenario. The maximum global bending moment is very high and the maximum shear force is at the same position as the maximum bending moment in the middle of the cargo hold. The ultimate strength and the damage mode of the structure depend on the soil characteristics and the ground geometry. Similarities in all presented midship scenarios are the main transverse fold/fracture in the double bottom and side structure as well as at least one longitudinal fold in the bilge structure. The transverse folds are mainly due to the bending moment whereas the isotropic buckles in the side structure and the compression of the bilge structure (longitudinal folds) are 
caused by shear forces. After $1 \mathrm{~m}$ of surface drawdown the models are outside the ideal elastic material range. In the plastic range it is valid that the longer the contact area is the higher the moments that can be absorbed by the structure. No significant cargo forces decrease the influence of the contact forces. The interaction of the contact forces with the hogging bending moment affects the longitudinal resistance of the hull. In all presented cases the ship structure reaches its ultimate hull capacity and collapses. The ultimate bending moments are all below the averaged UHGS value of $1.18 \mathrm{E}+07 \mathrm{kNm}$ predicted by classification societies (see table 2.8 in section 2.8). Thus during stranding amidship the structure collapses clearly before the class permissible capacity is reached. In the postcollapse stage all variational calculations are completely in contact with the ground.

The rigid ground is not a conservative border for soil characteristics. The soft ground simulations reaches earlier and lower values for the ultimate strength and results in more severe damage.

The bending moment curve given over the surface drawdown for different soft ground geometries can be estimated if one simulation is solved because the curves are qualitatively equal. The larger the ground plateau and the smaller the slope angle the higher the ultimate strength, whereby the detailed failure mode is different in each case.

The FE simulation Sa7p2 has almost the same damage in the side and bottom structure as the container vessel Fowairet. The other results of $\mathbf{S a 7 p 2 ~ s h o u l d ~ b e ~ v a l i d ~ f o r ~ t h e ~ i n c i d e n t ~}$ of Fowairet.

Stranding in the bow results in the second highest degree of damage. The damage is almost exclusively caused by the shear forces. For the stern scenario the damage is more or less insignificant. For the bow and stern scenarios the rigid ground is a conservative border. The effects of different ground geometries on the hull capacity and the collapse mode are comparable to the midship scenario. Calculating stranding in the stern section gives only a little more information than the estimation with the pure beam model, whereas stranding in the bow region results in an unexpected early failure of the outer shell plating. The degree of collapse cannot be concluded from the height of the external forces and moments.

Comparing the three stranding positions by normalising the absorbed moments and shear forces it becomes clear that the smaller the normalised moments and shear forces the greater the degree of damage, whereby the ability to absorb the global moments is more decisive with regard to the degree of collapse. The more severe case of each position reaches smaller normalised moments but higher normalised shear forces than the case with less damage.

\subsection{Conclusion}

In this chapter a simulation method is introduced for calculating stranding scenarios. Until now there are no methods to calculate stranding incidents with respect to all important factors. The method is based on the physics and mechanics of the phenomenon. The damage process is realistically simulated and the method is applicable to all ship types, 
ground geometries and many soil characteristics. The method can simulate real stranding incidents with very satisfactory results.

Neither the ship nor the ground nor the external forces are simplified. All referenced works simplify at least one of the three factors. The method is based on true loading conditions that are gained from hydrostatic calculations. The chosen ship is completely modelled and the ground is simulated with a well-known ground model. For the purpose of stranding simulations the Mohr-Coulomb model is sufficient.

The verification of the ship and ground model shows that the method gives reasonable results. The advantage of the method is that the local and global damage of the structure can be examined as a function of the soil characteristics, ground geometry, the position of stranding and the ebb tide. It is also possible to simulate multiple tide cycles. The method is very insusceptible to modifications and robust so that modifications or even simplifications to achieve, e.g. a faster calculation time, do not cause a significant loss in the quality of the results.

The presented scenarios underline the importance of research on the field of stranding. Most scenarios end in global damage to the structure. The degree of damage and the damage mode depend on the ground geometry and the soil characteristics. It is necessary to model the ground with a sand material model because the hull girder is not always less prone to hull collapse if set onto a soft bank. To improve accident prevention measures, the ground cannot be simplified to be rigid.

Furthermore, it is shown that real incidents can be calculated with very good conformance. The damage caused by the stranding of the Fowairet is reproduced by applying the method even though not many data are known.

Depending on the stranding position the bending moments and shear forces have a different influence not only on the damage but also on the hull capacity. In the following chapter a special focus is placed on the influence of the shear forces. A simplified method is presented to find moment-shear force interaction curves. Those curves are compared to the results of stranding scenarios to verify the influence of the shear force on the ultimate hull girder strength at different cross sections. 



\section{Ultimate load calculation}

The influence of the shear force on the ultimate bending moment for diverse cross sections under extreme loading conditions is achieved by simplified finite-element calculations. Moment-shear force interaction curves are gained to predict the reserve of the ultimate capacity under combined loading.

A pure bending moment experiment of a box girder is recalculated with the FE method. When the results of the FE method are adequately similar to those of the experiment the shear force is introduced into the model. In this way the procedure used in the FE method is verified and a realistic moment-shear force interaction curve can be found.

The box girder, which represents a very simplified single-hull tanker ship, is replaced by an open box girder. The open box girder is a major simplification of the midship section of Postpanmax. Its moment-shear force interaction curves are compared to two formulas known from the literature.

Then the simplifications are further reduced and moment-shear force interaction curves for the three slightly simplified cross sections- stern, midship, bow- already known from chapter 4 , are given. The cross sections consist of all main structural elements.

In the next step the ultimate hull girder strength of the simplified, fully modelled cross sections and of classification societies are compared. The determined interaction curves are opposed to those of the equivalent fully modelled cross sections. Finally, the influence of true loading conditions (during stranding) is analysed and compared to the fictive load cases.

\subsection{Recalculation of box girder experiments}

Three specimens (number 31, 22 and 23) of the experiment described in section 2.4.2, are recalculated with the FE method to validate the computational procedure. The geometry of the specimens and the setup are rebuilt. In the following three specimens of the experiment and their results are referred to as specimen 31,22 or specimen 23 whereas the FE results are referred to as model 31, 22 or model 23.

All models are built with the fully integrated shell element (type 16) and the material model described in section 4 is built without a failure criterion. The yield stress is constant over the entire model and corresponds to the yield stresses measured in the experiment (table 2.10). This applies for all models presented in this chapter.

The geometry of the three models is given in section 2.4.2. The boundary conditions are applied at the nodes of the lower flange of the HEB structure (European wide flange 
beams). In each calculation time step of $0.1 \mathrm{~s}$ a displacement of $0.3 \mathrm{~mm}$ is brought into the model. The total displacement is $30 \mathrm{~mm}$ at each side.

\subsubsection{Model 31, 22 and 23 under pure bending moment}

All models collapse because of buckling in the top plate. The resulting deformation and the ultimate strength depend on the material model, the element size, the time step size, the pre-deformation and the initial stress.

The material model is given by the experiment. The element size is optimised in respect of the computing time and the results. The pre-deformation and the time step size are varied for model 31 to find the sensitivity of the results and to save modelling and computational time. These calculations reveal that it is also necessary to introduce initial stress to get satisfactory agreement with the experimental results.

\section{Model 31}

\section{Pre-deformation and time step size}

Model 31 is calculated with three different pre-deformation conditions of the top plate: no deformation, a circular pre-deformation and the original pre-deformation given in the paper (Reckling et al. [59]). Figure 5.1 shows the pre-deformation and the end-deformation measured close to the ultimate bending moment and the division of the top plate into five areas for the upcoming discussion.
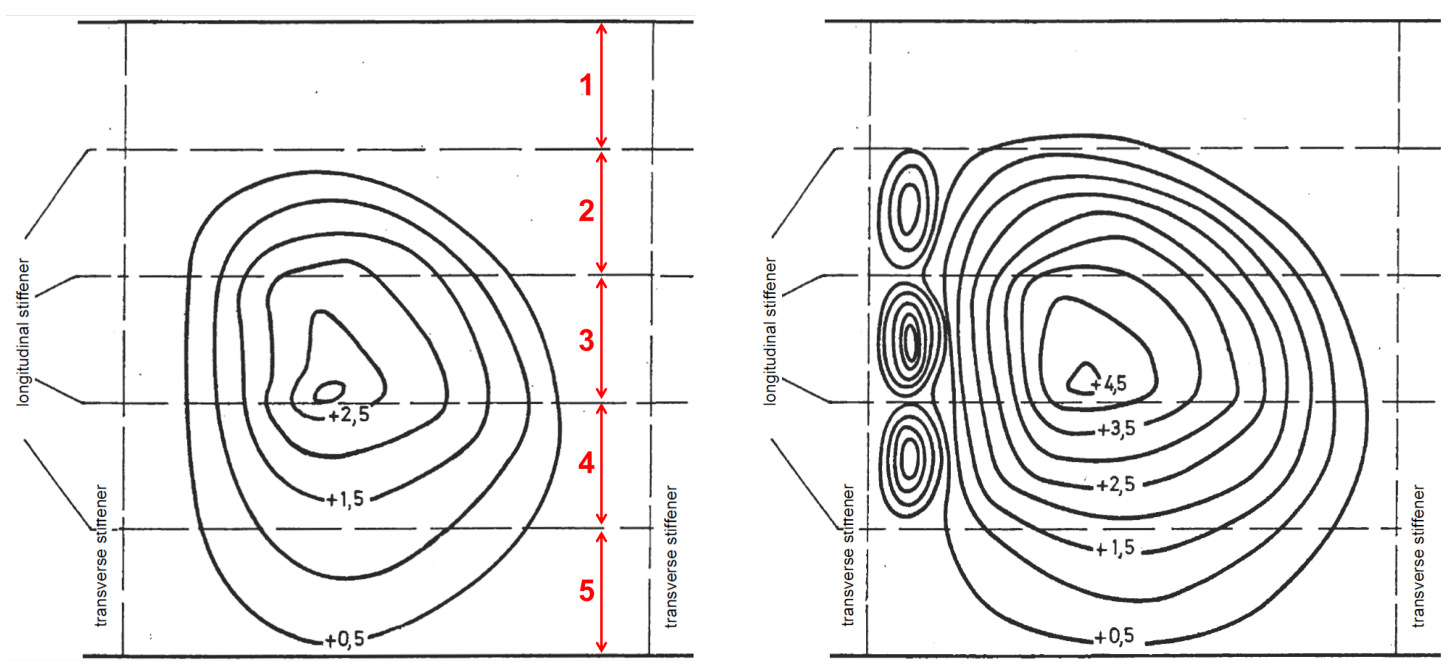

Figure 5.1.: Specimen 31: pre- and end-deformation in [mm] (Reckling et al. [59]).

In all three cases the buckling picture after reaching the ultimate strength as well as the ultimate strength itself differ. The following figures 5.2 (a), (b) and (c) show the displacement in z-direction in $[\mathrm{mm}]$ of the top when the ultimate strength is reached. The colour scale is fitted to the top plate and the node with the maximum displacement is also shown. 
If no pre-deformation is applied buckles establish between all stiffeners in the middle of the top plate (see figure 5.2 (a)). Whereas area 2 and 4 buckle in positive, the areas 1, 3 and 5 deform in negative z-direction. The maximal displacement is at node number 2 in area 3 that lies in the symmetry plane.

For the circular pre-deformation one buckle between each stiffener develops as shown in figure 5.2 (b). They are located at the edge of the circular pre-deformation. Now the deepest buckle is in area 4 with a displacement at node 4159. The outer areas (1 and 5) deform in positive z-direction.

The deformation of the model with the original pre-deformation, given in figure 5.2 (c), is similar to this with the circular pre-deformation. The position of the buckles can be distinguished by the different geometry of the pre-deformation. The maximal buckle occurs in area 3 . In contrast to the model with the circular pre-deformation only area 1 buckles in positive z-direction.

The collapse mode of the model with the original pre-deformation is more similar to the experiment, although the buckles between the stiffeners develop on the left side of the pre-deformation in the experiment.

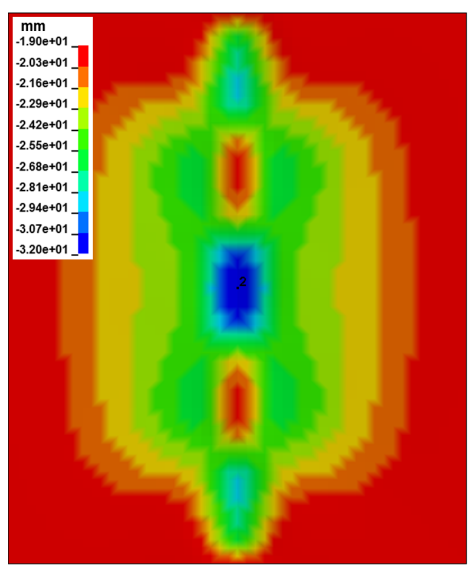

(a) no pre-deformation

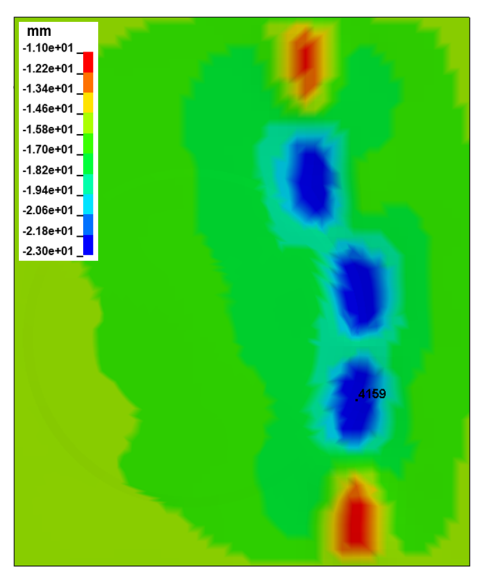

(b) circular pre-deformation

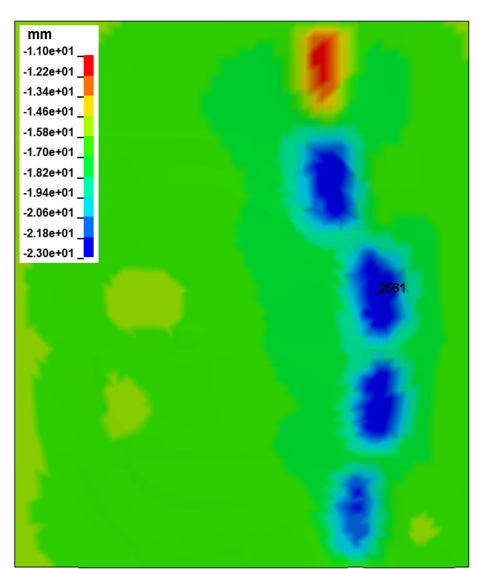

(c) original pre-deformation

Figure 5.2.: Model 31: end-deformation with different pre-deformations.

Figure 5.3 plots the bending moment $M_{h}$ of the model measured at the middle cross section $\mathrm{x}=0 \mathrm{~m}$ over the displacement $\mathrm{f}$ at $\mathrm{x}=0 \mathrm{~m} ; \mathrm{y}=0 \mathrm{~m}$.

The gradient of all curves is the same until the ultimate bending moment is reached. But the gradient of the moment-displacement curve for the experiment is different. The highest moment is reached by the model without any pre-deformation and the lowest by the calculation with the circular pre-deformation. The ultimate strength of the model with the original pre-deformations lies in between. It is $1.38 \%$ higher than the moment measured in the experiment, whereas the one of the calculation with the circular pre-deformation almost has the same height (see also table 2.10).

All curves have a jump because the buckles deepen suddenly, from one to the next time step. But using a smaller time step cannot significantly reduce the sudden decline. The buckling picture and bending moment are similar and only the computational time increases. For the moment- shear force interaction the maximum bending moment and 
shear force are needed. The post-buckling phenomenon is not considered here. Thus for all following simulations the time step size is not changed.

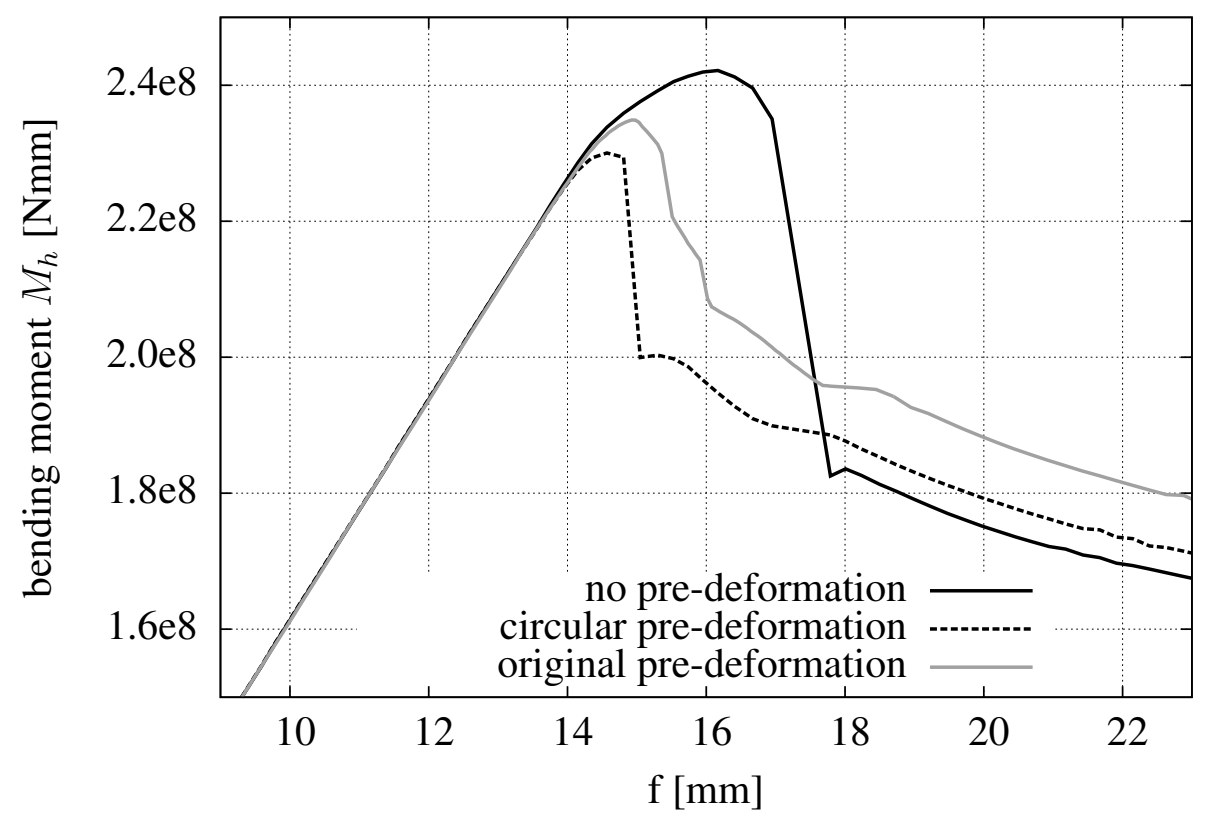

Figure 5.3.: No, circular and original pre-deformation: moment-displacement curves.

\section{Initial stresses}

The gradient of the moment-displacement curves in figure 5.3 are identical to the gradient of the ideal case. In the ideal case the material model is ideal elastic and no predeformation is applied. The moment-displacement curve in the ideal case is similar to the curve of the hypothetical girder until the girder starts to plasticise.

As stated by Reckling et al. [59] the manufacturing-related initial stresses are partly reduced by the stress relief heat treatment so that a smaller gradient is seen in the momentdisplacement curves. To gain satisfactory results the model with the original pre-deformation is also calculated with initial stresses.

Initial stresses at the stiffeners close to the yield stress are introduced in $\mathrm{x}$-direction by pre-loading the stiffeners of the top plate with temperature. There is no closed analytical solution to calculate the initial stresses due to temperature introduction for complicated geometries as existing (see Radaj [57]). The procedure is simple and fast (see also Lehmann [34]). After the temperature solution the displacement is applied onto the model as described above.

In figure 5.4 four moment-displacement curves are plotted: ideal $\mathbf{3 1}$ for ideal case, specimen 31 for experiment, model 31 for original pre-deformation given in figure 5.3 and model 31 with initial stress. The gradient of the model with initial stresses is very close to the gradient of specimen 31 . The course of the moments is not identical but the maximum moment is exactly the same $(231.7 \mathrm{kNm})$.

The deformation of the experiment is overlaid on the results of model 31 with initial 
stress in figure 5.5. The buckling picture shows a good conformance with the results of the experiment. The particular buckles are on the left side of the pre-deformation and the deepest deformation also occurs in area 3.

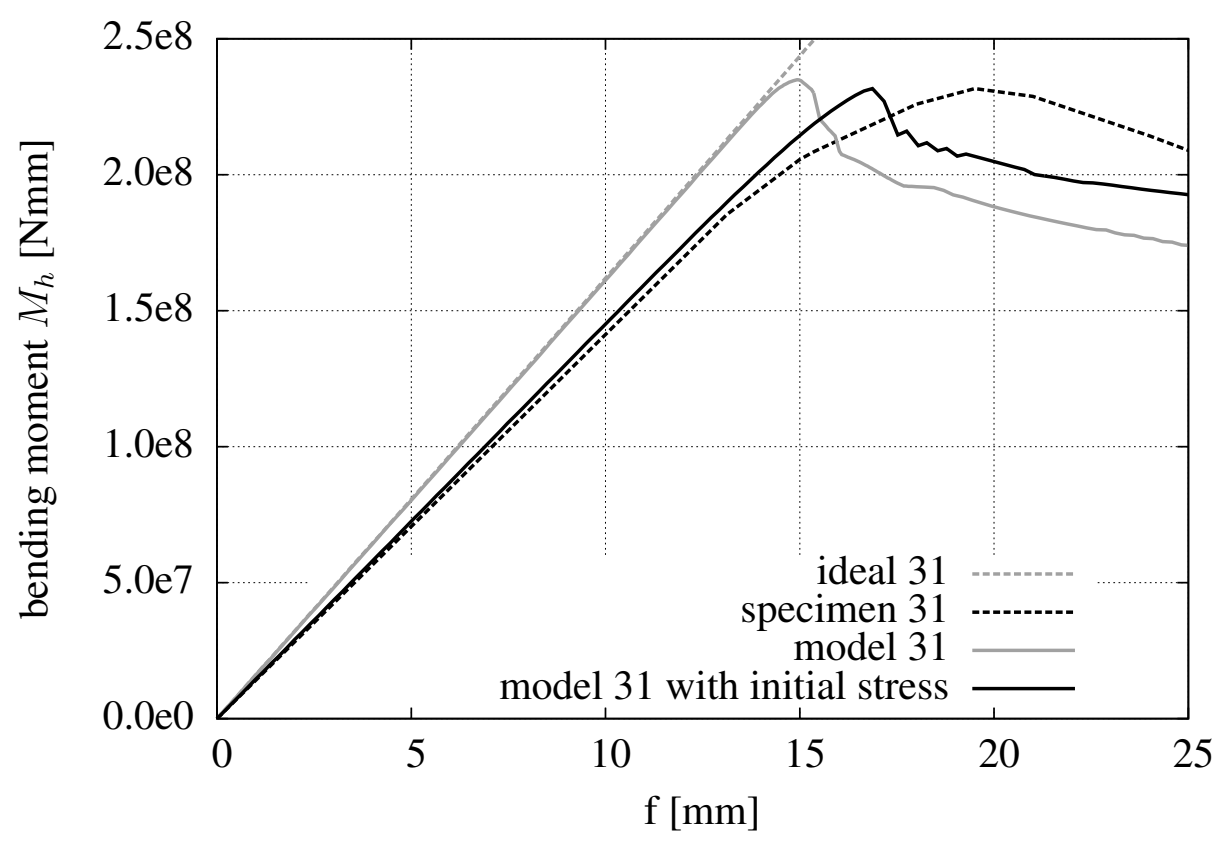

Figure 5.4.: Model 31 and specimen 31: moment-displacement curves.

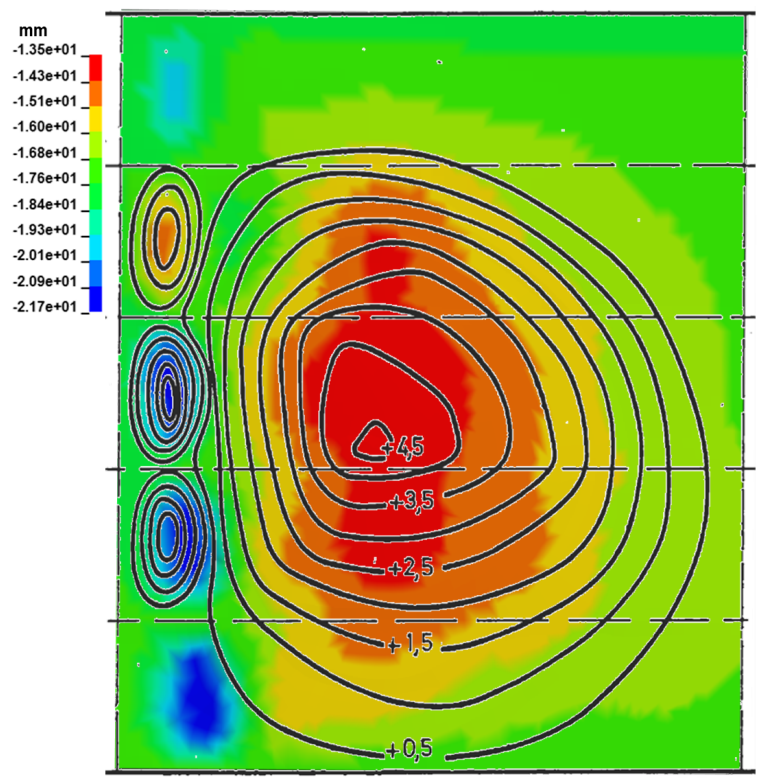

Figure 5.5.: Model 31 with initial stresses and specimen 31: end-deformation.

The FE results are satisfactorily equal to those of the experiment. The FE model is thus validated by reality and further calculations give reasonable results. There is no difference for the relation of moment and shear force if simulated with or without initial 
stress (see Paik et al. [51]). To save computational effort the initial stresses are not applied in the following calculations.

\section{Model 22 and 23}

Model 22 and model 23 are calculated with the original pre-deformation and without initial stress. The moment-displacement curves of all three models are given in figure 5.6. The cross section of model 22 and model 23 are equal and stiffer than model 31. Model 22 is shorter than model 23 and model 31. The effect of different cross sections and lengths between the bulkheads can be re-found in the height of moment-displacement curves. The stiffest model (model 22) reaches the highest maximal moment. The decrease of the moment curves are much smoother for model 22 and model 23 than for model 31. The collapse of the top plate does not occur so suddenly, which can also be seen in the buckling pictures. Model 22 and model 23 are stiffer than model 31 and therefore the total collapse takes more time. The curves of specimen 22 and specimen 23 do not decrease. All curves gained by FEM decrease after they reach the ultimate bending moment. The maximum bending moment of model 22 is $3.4 \%$ higher than in the experiment. And model 23 reaches $2.17 \%$ less of the experimental ultimate moment. Model 22 and model 23 also have a different gradient of the moment-displacement curves.

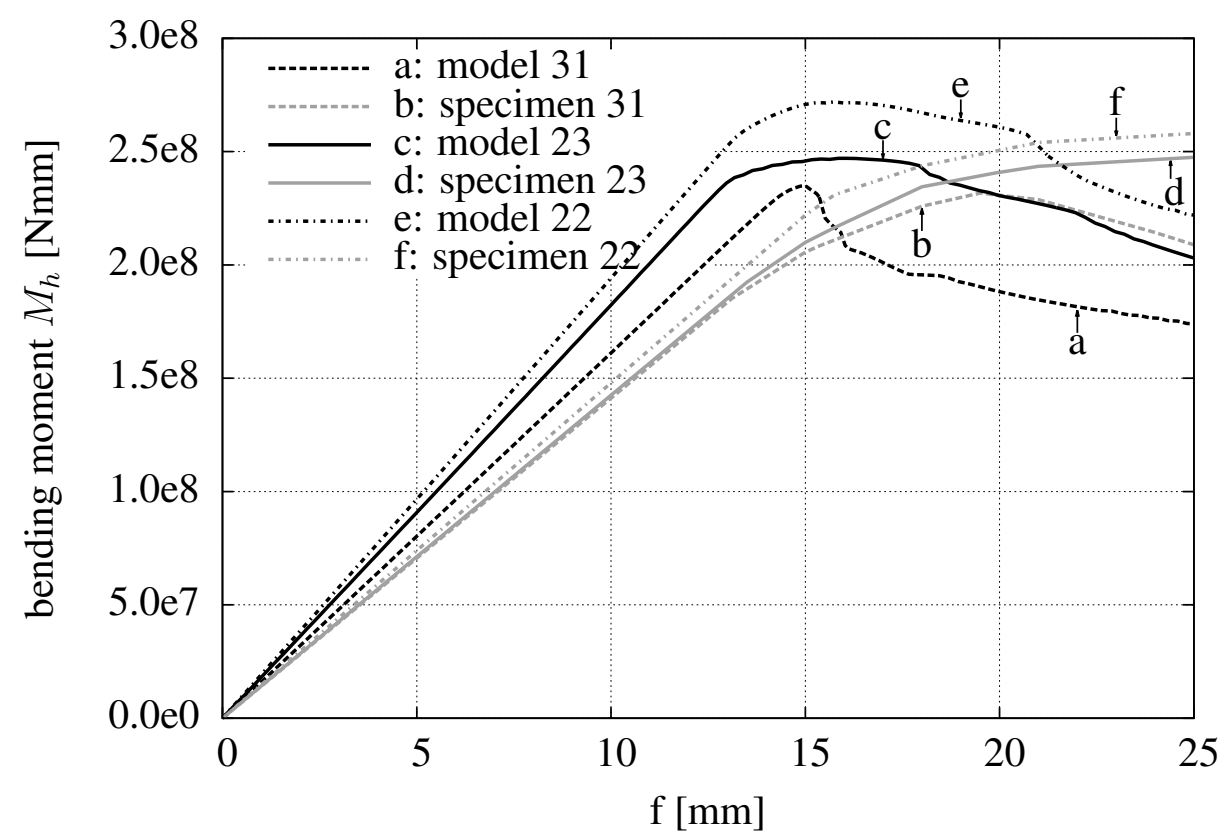

Figure 5.6.: Model 31, 22 and 23: moment-displacement curves.

The differences between specimen 22 and specimen 23 to model 22 and model 23 are comparable to those between specimen 31 and model 31. 


\subsection{Moment-shear force interaction of box girders}

To introduce the shear force two additional load cases are used. The pure bending load case of the experiment is called 'lc 1'. In 'lc 2' only one force is acting, whereas in 'lc 3' the second force operates in the opposite direction. Figure 5.7 shows the relation between the bending moment and the shear force and table 5.1 gives the relative size of force P2 for all presented cases.
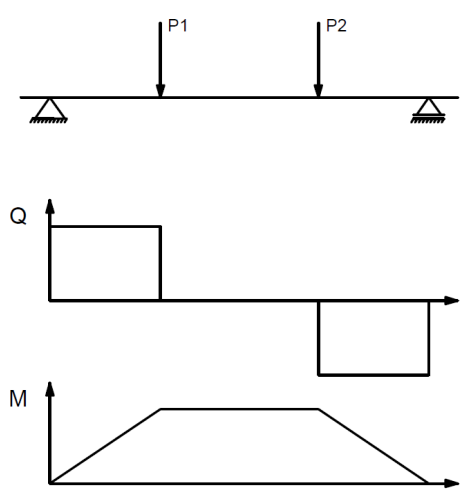

(a) lc 1
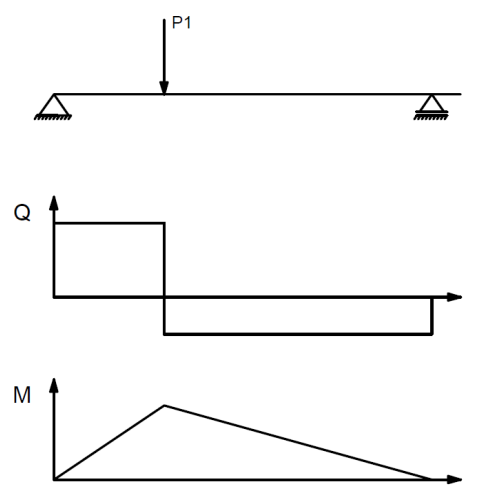

(b) lc 2
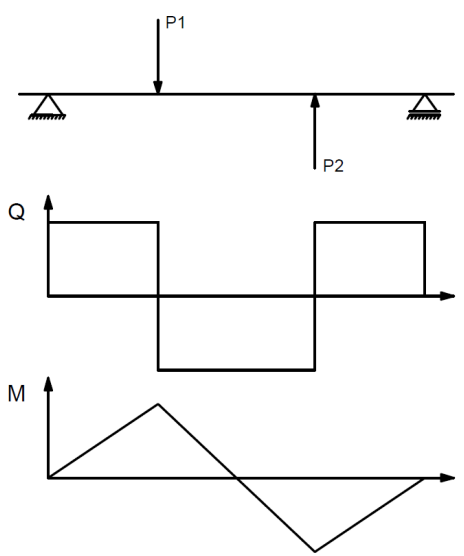

(c) 1 c 3

Figure 5.7.: Load cases 1-3

Table 5.1.: Load cases.

\begin{tabular}{lrr}
\hline lc & force P2 & comment \\
\hline 1 & $1 \cdot \mathrm{P} 1$ & pure bending \\
$1 \mathrm{a}$ & $1 / 2 \cdot \mathrm{P} 1$ & \\
2 & $0 \cdot \mathrm{P} 1$ & \\
3 & $-1 \cdot \mathrm{P} 1$ & pure shear force \\
$3 \mathrm{a}$ & $-1 / 2 \cdot \mathrm{P} 1$ & \\
\hline
\end{tabular}

\section{Model 31}

Figure 5.8 gives the moment-displacement curves for each load case. The associated shear forces are plotted in figure 5.9. In both figures the evaluation points for the moment-shear force interaction curves are marked. At the displacement where cases 1, 1a and 2 reach the maximum moment the equivalent shear force is read out. In cases 3 and $3 \mathrm{a}$ the maximum shear force determines the evaluation points.

The maximum bending moments and shear forces are normalised via two different approaches. On the one hand they are normalised by their analytical plastic limit values given in equations (5.1), whereas $\sigma_{F}$ is the yield stress, $W_{p l}$ is the plastic section modulus and $A_{s}$ is the effective shear area built of the side shells. The plastic shear stress $\tau_{p l}$ is given among others by Reckling [58] or Petersen [56]. 


$$
\begin{aligned}
M_{p l} & =\sigma_{F} \cdot W_{p l} \\
Q_{p l} & =\tau_{p l} \cdot A_{s} \text { with } \tau_{p l}=\frac{\sigma_{F}}{\sqrt{3}}
\end{aligned}
$$

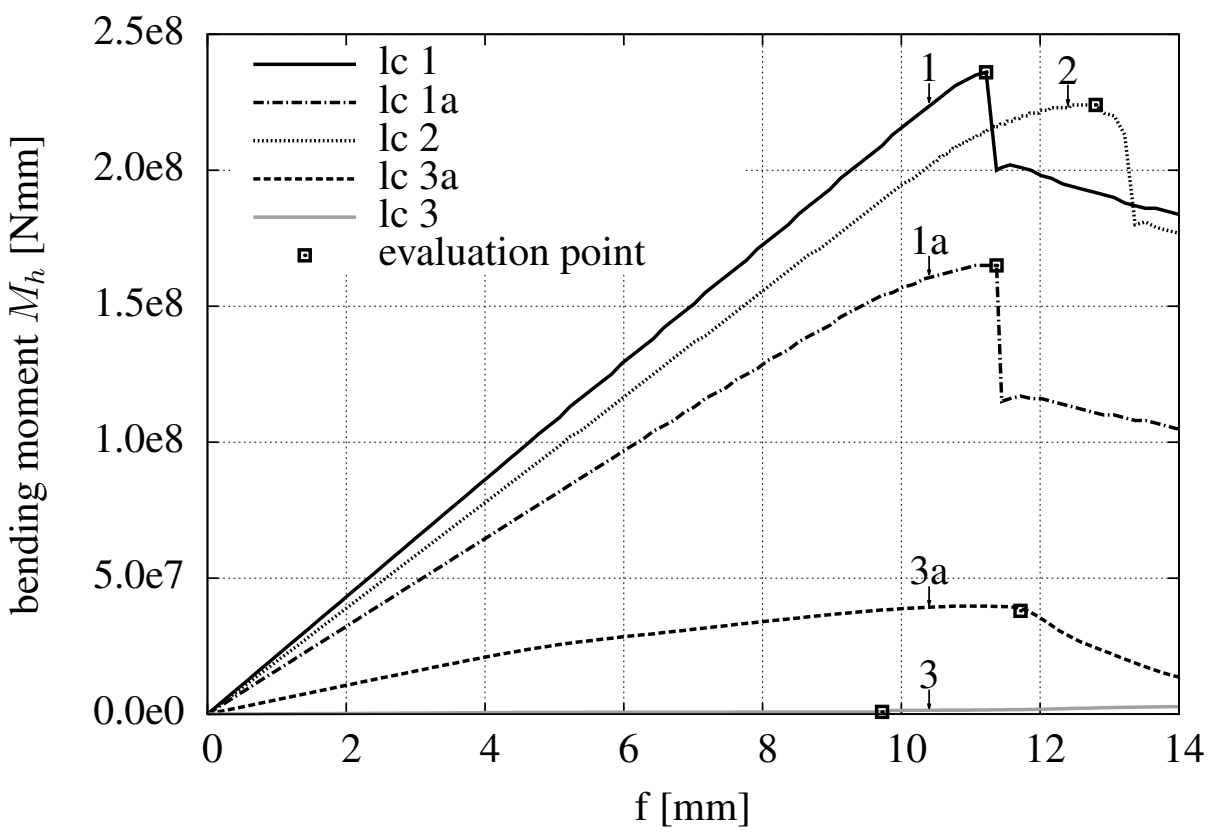

Figure 5.8.: Model 31: moments of of load cases 1, 1a, 2, 3, 3a.

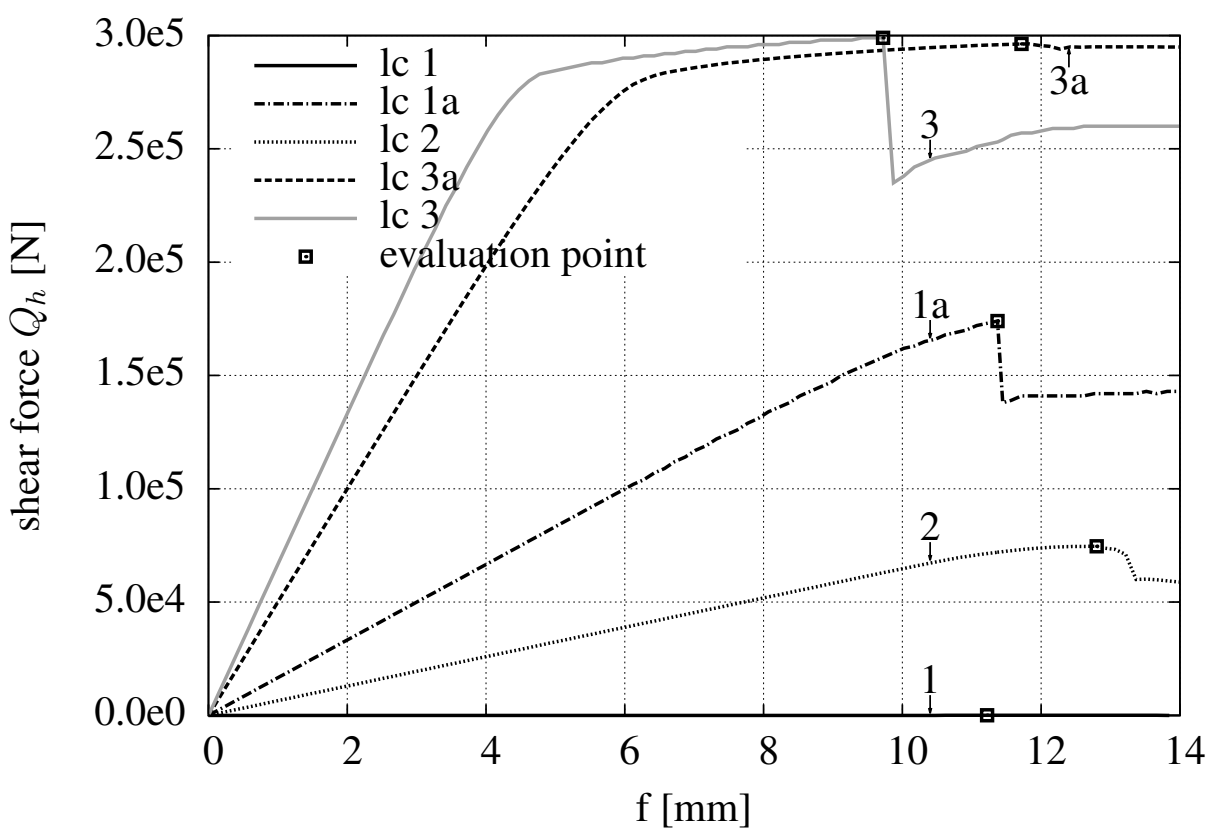

Figure 5.9.: Model 31: shear forces of load cases 1, 1a, 2, 3, 3a. 
On the other hand, the ultimate capacity of the girder under pure bending moment $M_{V u}$ and pure shear force $Q_{V u}$ is calculated by the formulas of Paik et al. [51], given in section 2.4.2 and called 'P-M'. The following figure 5.10 gives three moment-shear force interaction curves. The two continuous lines are based on the evaluation points of figure 5.8 and 5.9. They are normalised either by their plastic limit $M_{p l}$ and $Q_{p l}$ (black) or by $M_{V u}$ and $Q_{V u}$ (grey). The third line is the result of equation (2.14) (P-M equation) for the combined loading. It starts and ends with 1 because it is normalised by its own values $M_{V u}$ and $Q_{V u}$. Additional variations of lc 1 are calculated to get sampling points between lc $1 \mathrm{a}$ and 2 . Those additional cases lie exactly on the connection line between lc 1a and 2, which is why they are disregarded.

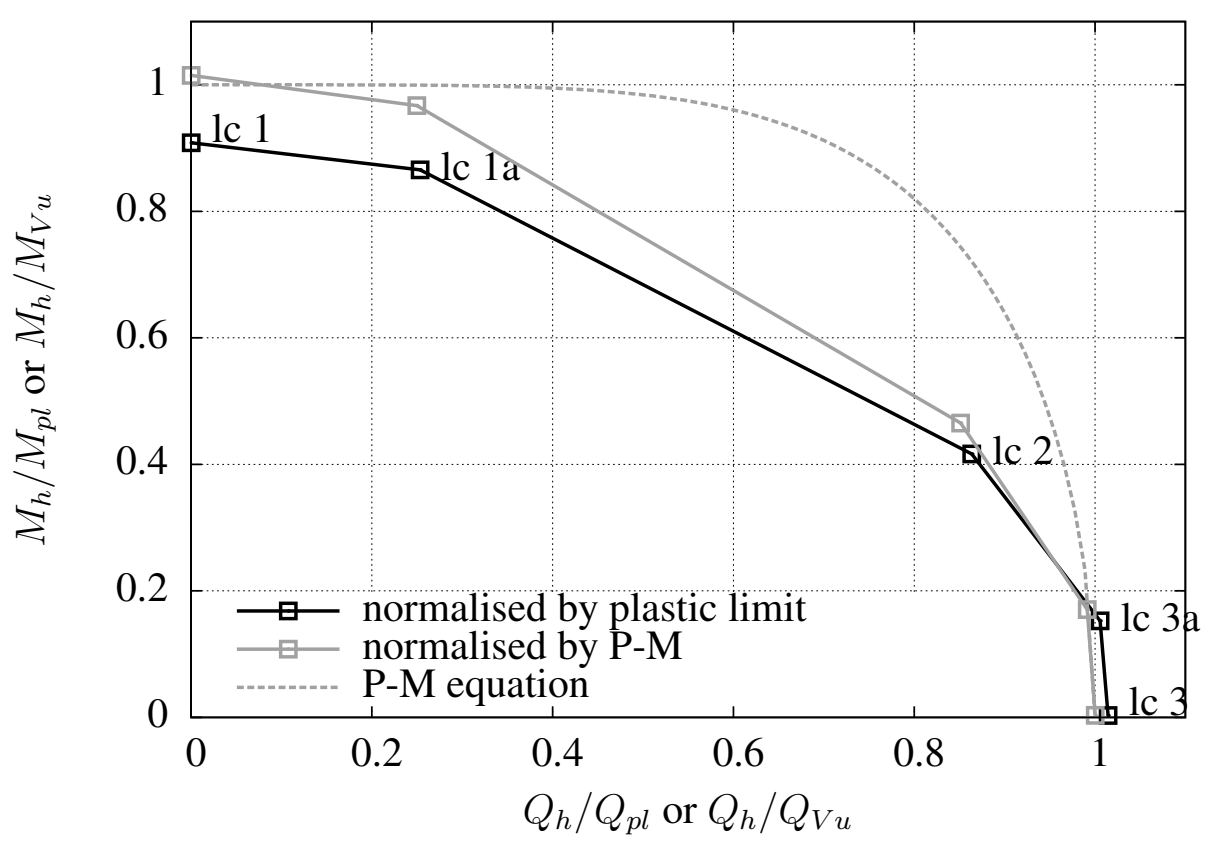

Figure 5.10.: Model 31: moment-shear force interaction curve.

The formula for shear force $Q_{V u}$ of Paik et al. [51] result in the same value as for FE lc 3. The ultimate bending moment $M_{V u}$ is slightly smaller than in lc 1. Equation (2.14) does not give adequate results for the combined load case of bending moment and shear force. The area under the curve is also larger resulting in an overestimation of the capacity.

\section{Model 22 and 23}

The moment-shear force interaction curves for model 22 and model 23 are given in figure 5.11 together with model 31. All examination points of the load cases are normalised by their plastic limit $M_{p l}$ and $Q_{p l}$. In addition the points of $M_{V u} / M_{p l}$ and $Q_{V u} / Q_{p l}$ for model 22 (P-M 22) and model 23 (P-M 23) are plotted.

Model 22 has the highest moment-shear force interaction curve and model 31 the lowest. The curve of model $\mathbf{2 3}$ again lies in between the two other models (compare figure 5.6). If more stiffeners are used in the side, bottom and top plate, higher normalised forces 
and moments can be carried by the structure. The stiffer the cross section the higher is the moment-shear force interaction curve although the values are normalised by their geometric specifics. The curves of model 22 and model 23 are qualitatively similar but the numeric quantity values are different due to unequal model lengths. Not only diverse cross sections lead to a different moment-shear force interaction but also different lengths of the models.

In load case 1 all values are close together and lie below the plastic limit moment. The ultimate capacity of the girder is not equal to the full plastic capacity due to the predeformation and the post buckling behaviour.

Whereas all values of $Q_{l c 3} / Q_{p l}$ are higher than the plastic limit. Apparently the shear force is not only carried by the side shell as given in equation (5.1).

The formulas of Paik et al. [51] cannot appropriately predict the ultimate capacity especially for loading with pure shear force. Therefore, upcoming curves are normalised with their plastic limit values.

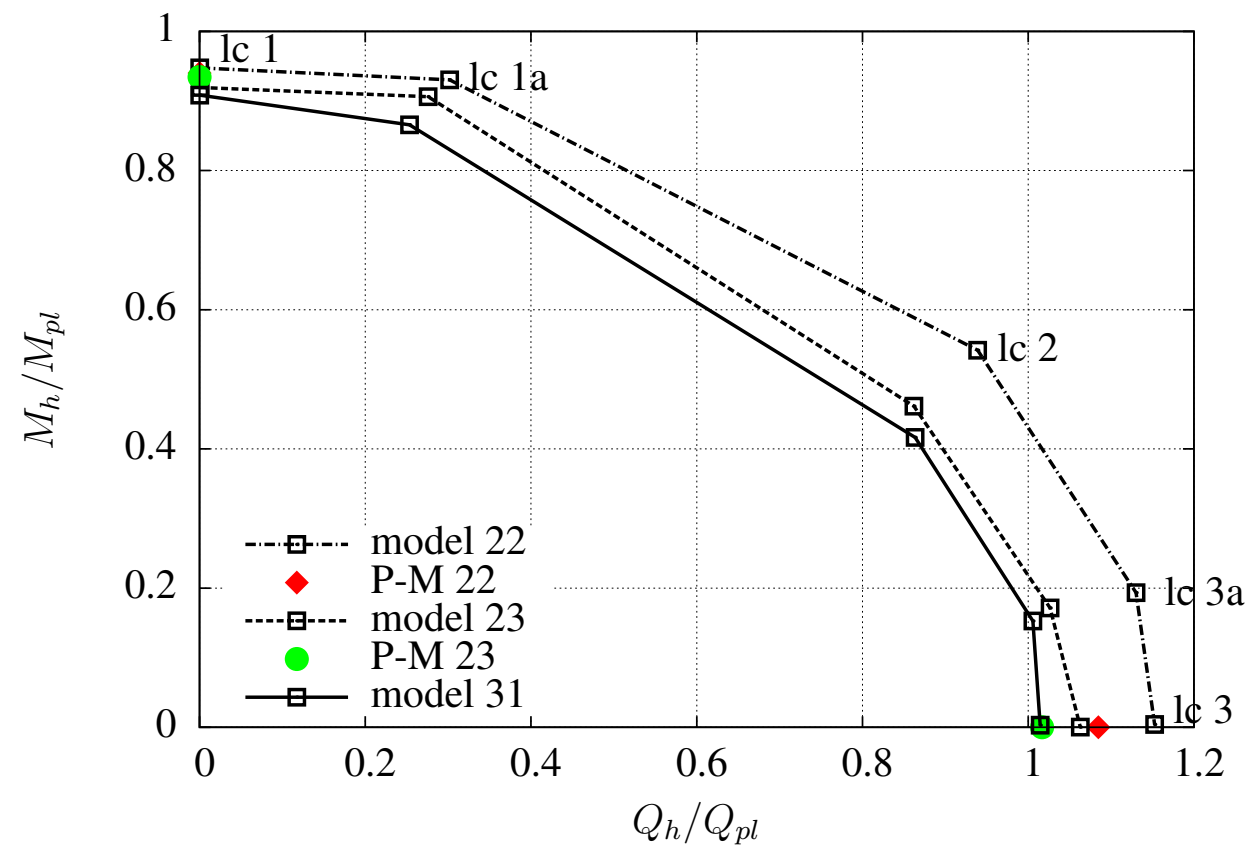

Figure 5.11.: Model 22, 23 and 31: moment-shear force interaction curve.

\subsection{Moment-shear force interaction of simplified cross sections}

The interaction curves of the box girder (model 31) is compared to curves of an open box girder and the simplified midship section of the vessel Postpanmax.

The tanker vessel-like cross section from the experiments of Reckling et al. [59] is replaced by a major simplification of the Postpanmax midship section (open box). Minor modifications at the connection of HEB-400 and subcarrier are needed and the model is turned so that hogging bending arises. The rest of the experimental setup is identical to 
that described above. The new cross section is shown in appendix C.1. The load cases of table 5.1 are applied to achieve the interaction curves.

For the open box girder it is better to apply external moments instead of node displacements. So for the following analyses of different cross sections the loading is introduced into the models via moments instead of displacements (see Janele [27]). The size of the external moments is chosen to achieve identical combinations of moment and shear force in the examination plane as given in figure 5.7 and table 5.1. They are applied at the particular height of the neutral axis at one master node. The master node is connected to the model's end via constrained equations so that the cross section ends remain planar. The HEB beam and the subcarriers are disregarded since they are only needed for the introduction of displacement. The open box girder is also calculated with the new setup and identical interaction curves are achieved as for the first setup.

The midship section and later the bow as well as the stern section are simplified cross sections (called simple mid CS, simple bow CS and simple stern CS). The dimensions are as built, all main structural elements and the frame shape are taken from the construction drawings of Postpanmax. The longitudinal stiffeners and the manholes are not modelled. In all three cases the model extends over three complete cargo holds. The frame shape does not change over the model's length. The simplified cross sections of the FE calculations are also given in appendix C.1.

All interaction curves are gained according to the procedure presented in section 5.2. The average calculation time for all models is 4 minutes on a common PC. Additionally at the examination point the longitudinal stress distribution and the buckling mode is analysed to guarantee the correctness. A detailed analysis is given in Janele [27].

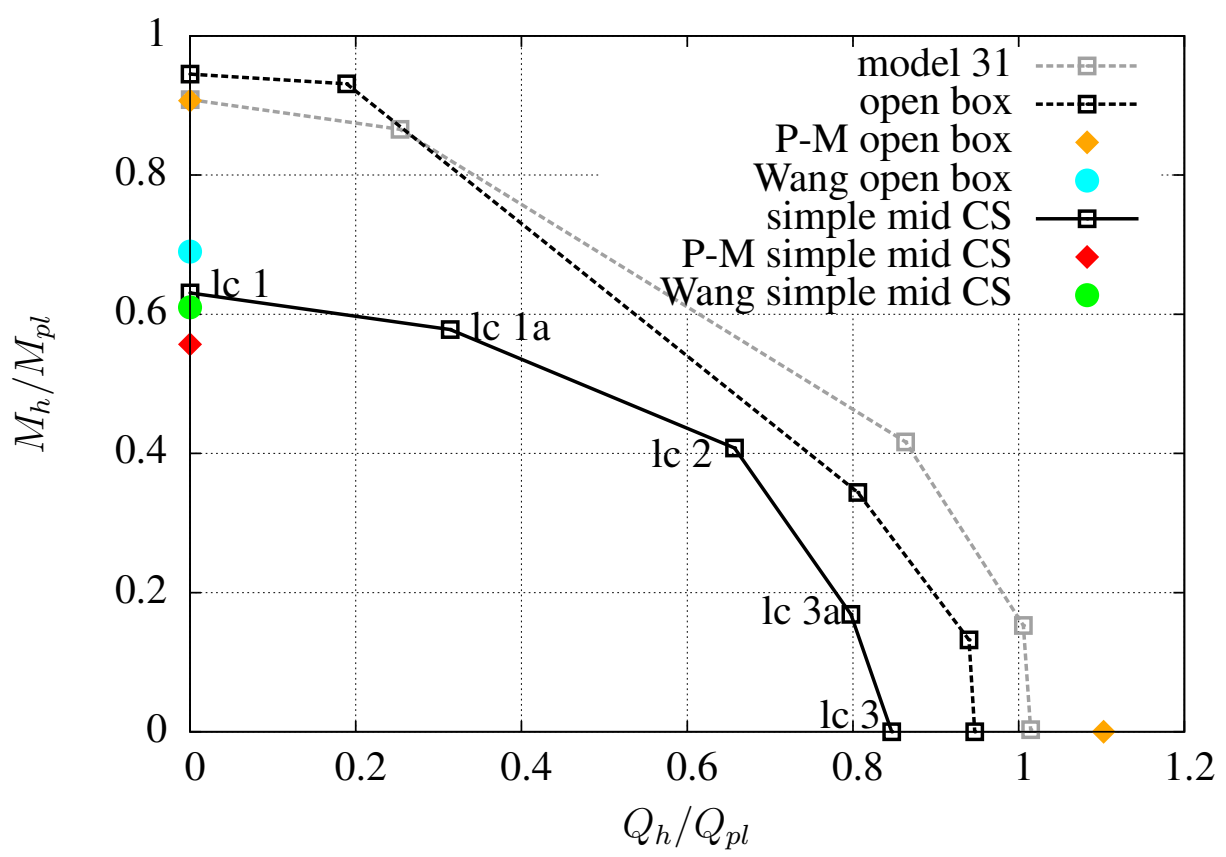

Figure 5.12.: Midship sections: moment-shear force interaction curve.

In figure 5.12 the interaction curves of model 31, the open box and simple mid CS are given. Furthermore, $M_{V u} / M_{p l}, Q_{V u} / Q_{p l}$ and the dimensionless section modu- 
lus $S M^{\prime} / S M^{0}$ given in equation (2.5) which is plotted for the open box girder. For the simplified midship section $Q_{V u} / Q_{p l}$ cannot be calculated. The simplified cross section has no longitudinal stiffeners and the longest plate field is between stringer s2 and s3. This results in $b^{\prime}=8730 \mathrm{~mm}$ needed for equation (2.9a) and in a negative square in equation (2.11).

Both the formulas of Paik et al. [51] and the equation of Wang [79] do not yield in reliable results. One time they fit and then they lie alongside the FE results. The formulas/ equation are not useful for calculating the ultimate capacity under combined loading.

The curves of the closed (model 31) and open box do not differ much. A closed cross section has a better shear flow and thus model 31 can sustain higher shear forces. The open box on the other hand can absorb a higher bending moment because of a stiffer double bottom and side structure.

The differences between the open box girder and the simplified midship section are mainly due to the very diverse slenderness ratios $\beta$ given in equation (2.9a). Due to the experimental sizes the plate thickness are comparable large. The slenderness ratios of a bottom shell plate in the open box girder and of the equivalent plate in the (simplified) cross sections are given in table 5.2. In addition the form factor of all three cross sections is listed.

Table 5.2.: Different midship sections: slenderness ratio and form factor.

\begin{tabular}{rrrr}
\hline & open box & simple mid CS & mid CS \\
\hline$\beta$ & 1.10 & 4.40 & 1.54 \\
$\alpha$ & 1.60 & 1.91 & 1.26 \\
\hline
\end{tabular}

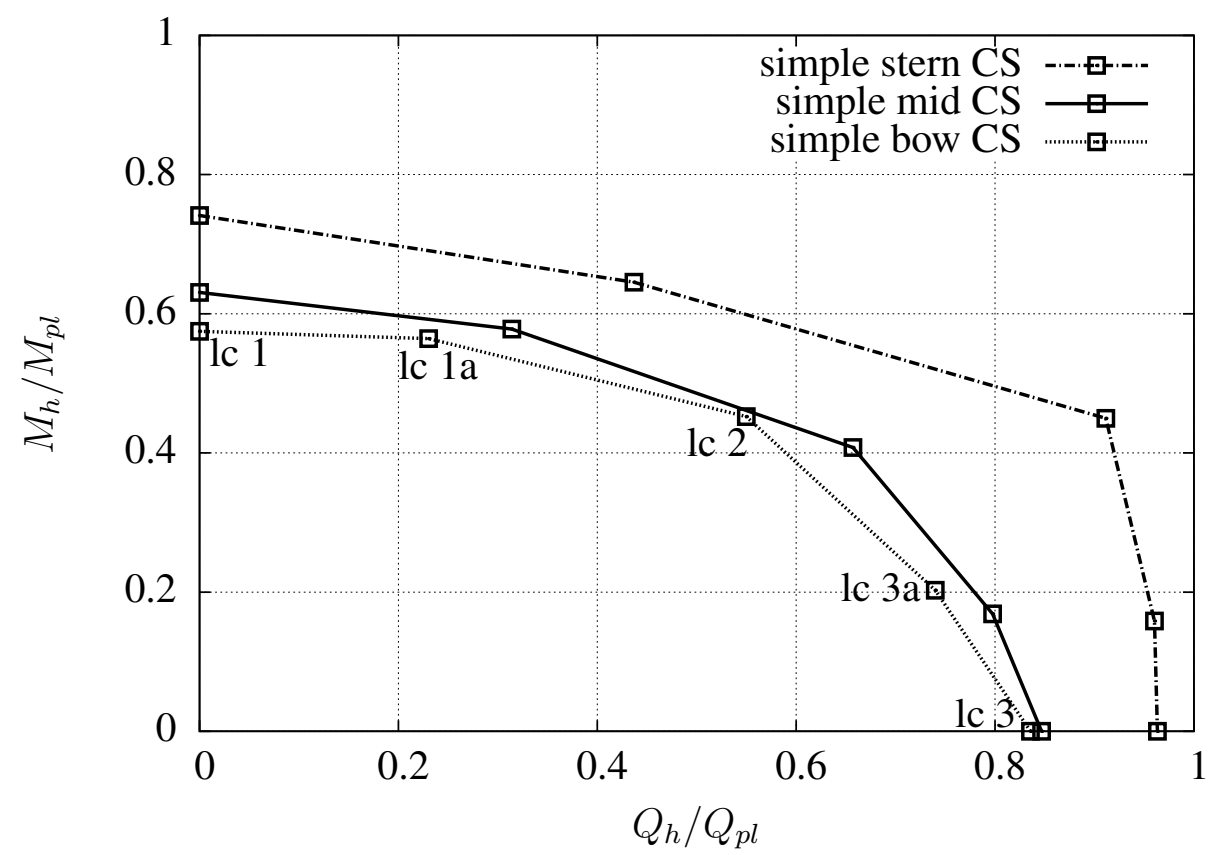

Figure 5.13.: Simple midship, bow and stern cross sections: moment-shear force interaction curve. 
In figure 5.13 the moment-shear force interaction curves of the three simplified cross sections are plotted. The stern cross section has the best ability to absorb high moments and shear forces. The stern cross section has a very stiff double bottom structure to compensate the weight from the main engine which results in a higher compressive resistance of the compression flange. The maximal shear force is almost the same as the perfectly plastic shear force $Q_{p l}$ due to the closed structure with two longitudinal bulkheads.

The curve of the bow section agrees with the curve of the midship section and lies slightly below it. The shear force can be compensated a little bit better than the pure bending moment. The step-wise closed side structure enables better shear flow, whereas the bottom structure is less stiff than the midship section.

In table 5.3 the area $\mathrm{A}$, shear area $\mathrm{A}_{s}$ and the normalised ultimate capacities of each cross section are summarised. The normalised ultimate bending moment $M_{l c 1} / M_{p l}$ and the normalised ultimate shear force $Q_{l c 3} / Q_{p l}$ can be given as a function of the cross section area. The moments lie on a straight line and the shear forces have a small bend at the midship sampling point (see figure 5.14).

Table 5.3.: Area of simplified cross sections.

\begin{tabular}{|c|c|c|c|c|}
\hline cross section & $\mathbf{A}\left[\mathbf{m}^{2}\right]$ & $\mathbf{A}_{s}\left[\mathbf{m}^{2}\right]$ & $\mathbf{M}_{l c 1} / \mathbf{M}_{p l}[-]$ & $\mathbf{Q}_{l c 3} / \mathbf{Q}_{p l}[-]$ \\
\hline stern & 6.24 & 2.03 & 0.74 & 0.96 \\
\hline midship & 4.60 & 2.25 & 0.63 & 0.85 \\
\hline bow & 3.56 & 1.79 & 0.57 & 0.84 \\
\hline
\end{tabular}

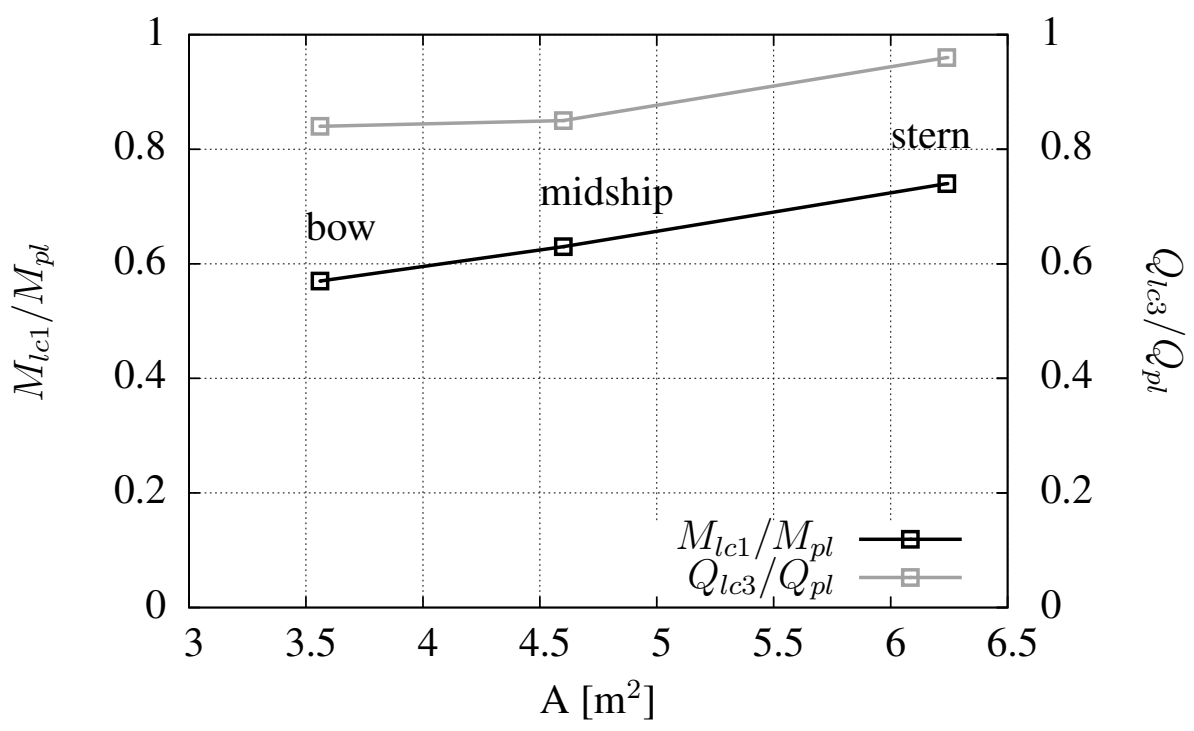

Figure 5.14.: Dependancy of ultimate capacity on cross section area.

If the ultimate bending moment $M_{l c 1}$ is known the ultimate shear force $Q_{l c 1}$ can be 
estimated and vice versa (see equation (5.3)). In equation (5.3) the ultimate bending moment $M_{l c 1}$ and shear force $Q_{l c} 3$ are now referred to as $M_{u}$ and $Q_{u}$ for universal use. The ultimate values $M_{u}$ respectively $Q_{u}$ are achieved when the cross section is only loaded with a bending moment respectively shear force.

$$
Q_{u}=\left(\frac{M_{u}}{M_{p l}}+0.2\right) \cdot Q_{p l}
$$

Once the ultimate capacity under pure bending and shear force is known the values for combined loading can be estimated via a modification of equation (2.14):

$$
\left(\frac{M}{M_{u}}\right)^{c_{1}}+\left(\frac{Q}{Q_{u}}\right)^{c_{2}}=1
$$

The values of the coefficient in equation (2.14) are proposed to be set to $c_{3}=2$ and $c_{4}$ $=5$. It is shown in figure 5.10 that those coefficients are not useful for the cross sections but they can be fitted to the particular curves. If, for example, the coefficients are set to $c_{3}=c_{1}=1.67, c_{4}=c_{2}=2$ and the maximal moment $/$ shear force of the FE calculations with the simple midship section are taken, reliable results are gained. Figure 5.15 shows a good conformance of both curves. The new coefficients also provide acceptable results for the simple bow section but for the stern section other coefficients are needed. Therefore, the coefficients $c_{1}$ and $c_{2}$ depend on the location of the cross section and are given for a container vessel in table 5.4. Cross sections that lie between the aft and the engine room front bulkhead, have a different construction and need other coefficients in equation (5.4).

Equation (2.14) may give a reliable moment-shear force interaction curve if the coefficients are adjusted, but the ultimate capacity for the load case pure bending or pure shear force of a cross section should not or in some cases cannot be calculated with the formulas given in the same publication (Paik et al. [51]).

Table 5.4.: Coefficient for equation (5.4).

\begin{tabular}{lrr}
\hline location of cross section & $\mathbf{c}_{\boldsymbol{1}}$ & $\mathbf{c}_{\boldsymbol{2}}$ \\
\hline aft engine room bulkhead & 1.67 & 2.00 \\
fore engine room bulkhead & 4.35 & 1.00 \\
\hline
\end{tabular}

According to figure 5.14 it is only necessary to calculate one ultimate bending moment or shear force for one cross section $c s 1$. The values for another cross section $c s 2$ can be calculated by equation (5.5). Equation (5.5) gives a conservative estimation of the ultimate shear force.

$$
M_{u c s 2}=\left(\frac{M_{u c s 1}}{M_{p l c s 1}}+\tan (\alpha)\right) \cdot M_{p l c s 2} \text { with } \alpha=35.31^{\circ}
$$




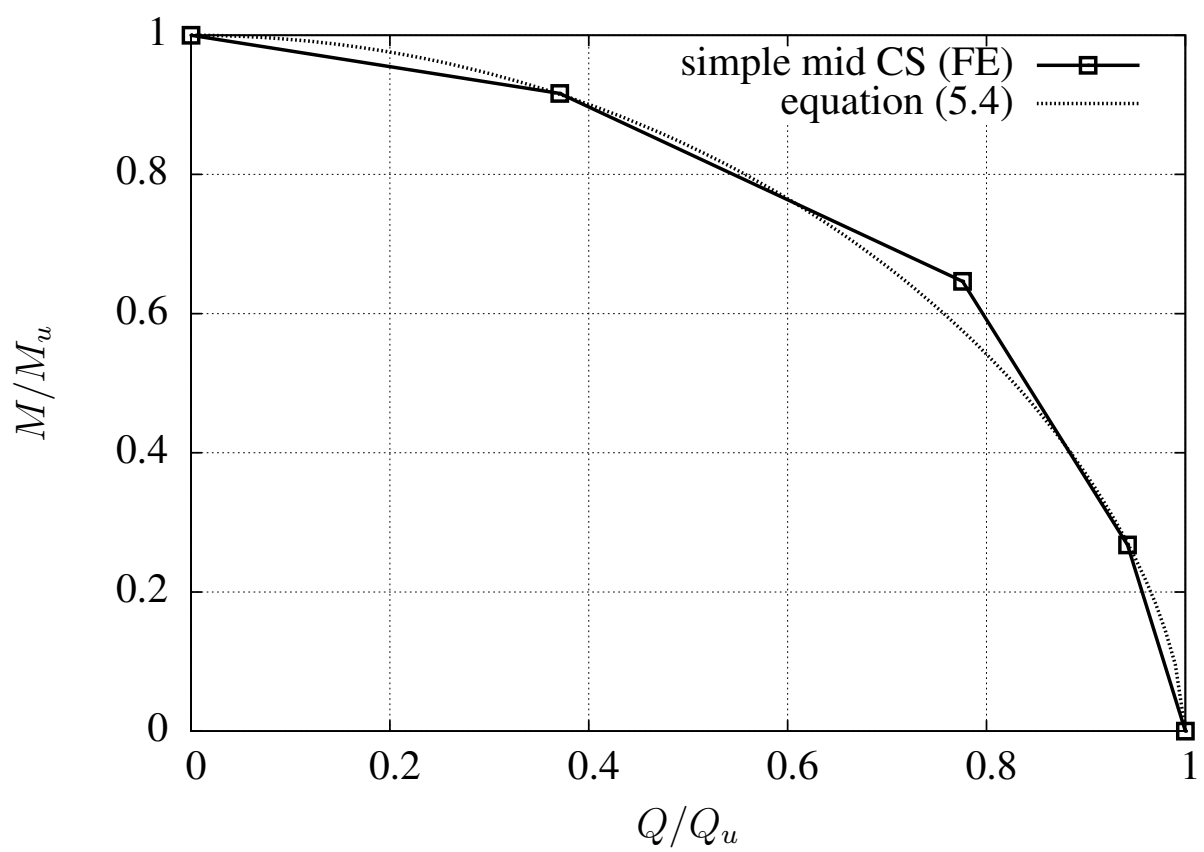

Figure 5.15.: Simple midship section: moment-shear force interaction curve.

\subsection{Comparing UHGS of simplified sections, stranding scenario and classification rules}

The ultimate hull girder strength of the vessel Postpanmax under combined loading is given in this work via different methods. The UHGS for pure bending is calculated with classification rules that mainly use Smith's method (see chapter 2). With two more simple FE calculations of the models used for the calculation of stranding scenarios in chapter 4 as well as with their results, moment-shear force interaction curves can be gained. Consequently the interaction curves of the simplified cross sections can be compared to curves of the same but not simplified cross sections in real loading conditions.

The shell part of the FE models (midship, bow and stern) of chapter 4 are also loaded with a pure bending moment. Secondly, the rigid ground is pressed into each model that is fixed at its ends so that a very small bending moment appears. Additional examination points for the interaction curve are taken from each conservative scenario with the ground geometry a7p2. The examination points are normalised by their plastic limit values.

The UHGS of the midship cross section, shown in figure 4.3, is determined according to different classification rules (see subsection 2.4.1). In table $2.8 M_{U, h o g}$ is given for hogging condition in pure bending and the averaged value is $M_{U, h o g}=1.18 \mathrm{E} 7 \mathrm{kNm}$. The rules of Germanischer Lloyd are taken for comparison because the complete vessel Postpanmax is implemented in POSEIDON. The UHGS calculated with POSEIDON is slightly higher $(1.19 \mathrm{E} 7 \mathrm{kNm})$ than the averaged value. The moments $M_{U, h o g}$ of the three cross sections are also normalised with the plastic limit values. 
In figure 5.16 the interaction curve of the simplified midship cross section is compared to $M_{U, h o g} / M_{p l}$ (GL mid CS) and the interaction curve gained with the fully modelled FE midship section called 'mid CS with eq. (5.4)'. The two black points at the ends of curve 'mid CS with eq. (5.4)' are the result of the additional FE calculation (pure bending moment and rigid ground pushed into fixed model). The curve is then assessed with the equation (5.4).

The UHGS predicted by the classification societies cannot be reached. $M_{U, h o g} / M_{p l}$ is more than $20 \%$ higher than the maximal bending moment of 'mid CS'. If the lowest value of $M_{U, h o g}$ in table 2.8, received with the rules of $D N V$, is taken for comparison, $M_{U, h o g} / M_{p l}$ is only $6 \%$ higher. The disadvantages and the simplifications of Smith's method are already described in section 2.4 and are the reason for the differences. Especially the dependency on the accuracy of the average stress-average stain relationship is essential. The finite element method is the most accurate method for progressive collapse analysis and is accepted of all classification societies as evidence of the UHGS.

Furthermore, the curve 'mid CS with eq. (5.4)' lies below the curve generated with the simple midship section. Although the simplified cross section has no longitudinal stiffeners it is not less stiff because they are also considered in the value $M_{p l}$. But the disregard of the manholes leads to a stiffer section for case simple mid CS.

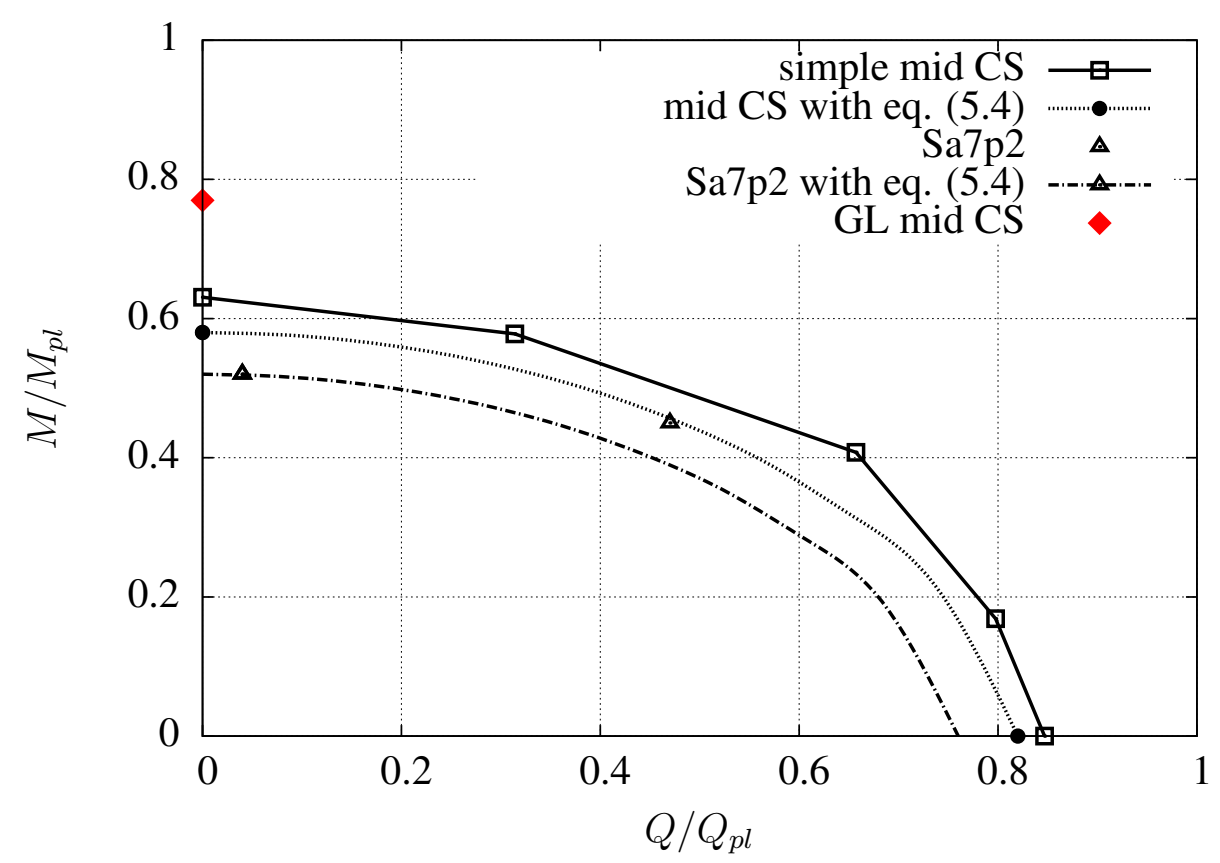

Figure 5.16.: Moment-shear force interaction curve of midship sections.

Figure 5.16 illustrates the influence of the high shear forces on the ultimate strength during stranding. The point of 'Sa7p2' (black triangles) are examination points taken from the collapse state of stranding scenario Sa7p2. The other points also lie on that line and are therefore not given. The curve 'Sa7p2 with eq. (5.4)' is assessed via equation (5.5) and modified equation (5.4) and is the final interaction curve for the midship section. It is a conservative interaction curve because one value is higher. $M_{S a 7 p 2} / M_{p l}$ is $10 \%$ smaller 
than $M_{\text {mid } C S} / M_{p l}$ and $17 \%$ smaller than $M_{\text {simple mid } C S} / M_{p l}$.

For the bow and stern section equal results as for the midship section are found. In appendix C.2 the graphs for bow and stern section are given. Only the final interaction curve together with the simple CS curve and the value of the classification society is plotted. The percentage differences to the $G L$ value is different. But the final curves have the same difference to the simple CS curves as in the midship section.

\subsection{Discussion}

If the pre-deformation is modelled as exactly as possible the resulting buckling mode is not automatically the same as in the experiment. Neither for the FE calculation nor for the experiment is it possible to predict the collapse mode of the girder due to the predeformation. The introduction of initial stress results in an identical maximum bending moment, a similar gradient of the moment-displacement curve and an equal buckling mode. The results of the calculation with the original pre-deformation and initial stress are satisfactorily equal to those of the experiment and further calculation with the additional shear force gives reasonable results.

Various load cases give the supporting points for the moment-shear force interaction curve. For three different box girders, one open box girder and three simplified cross sections of the vessel Postpanmax an interaction curve is gained. Comparison to the literature reveals that the curves are reliable. Not only different cross section but also different length of the models lead to a different moment-shear force interaction. For the open box girder a similar interaction curve is assessed. The curve of the open box girder is much higher than the one calculated with the equivalent simplified midship cross section because of very different slenderness ratios.

The interaction curves of the simplified cross section can be ordered starting with the maximum curve: stern, midship and bow. Their stiffness degrees and sectional areas have the same order. With the linear dependency of section area to ultimate strength and of ultimate moment to ultimate shear force a relation between the ultimate values can be found. Furthermore, the coefficients of an equation already referenced in literature are modified. Thereby different coefficients are needed for the stern section than for the rest of the ship due to its different construction. The proposed equations are not applied to other vessels. The tanker ship-like cross section (box girder) gives similar results to the container ship-like cross section (open box) suggesting that the procedure should be valid for other vessels, too.

The comparison of the simple sections to the equivalent fully modelled cross section shows that the cut outs decrease the stiffness of the section and thus the ultimate strength. In contrast to the fictive load cases, the true loading of the structure during stranding reduces the ultimate strength by $10 \%$. 


\subsection{Conclusion}

The described procedure to gain the moment-shear force interaction curve for a cross section is simple and validated. For any cross section of a ship a curve can be calculated using the presented procedure and the influence of the shear force on the ultimate hull girder strength during stranding can be evaluated.

The two proposed equations can be used to gain interaction curves for any cross section whereby only one ultimate value (moment or shear force) is needed. This value should be calculated with FE methods. The formulas from the literature do not give reliable results and the UHGS determined with classification rules overestimates the maximum bending moment.

A significant simplification of a vessels' cross section is only recommended for the calculation of moment-shear force interaction curves if equal plate slenderness ratios are used. When simplified cross sections are used the interaction curves should be corrected by $20 \%$ to obtain a conservative estimation. Thereby are $10 \%$ for the differences of simplified and non-simplified cross section and $10 \%$ for the difference of fictive and real combined loading conditions. The shear force reduces the ultimate hull girder strength during stranding by $10 \%$ in all ship sections. 


\section{Conclusions}

This work contributes to the design of vessels against accidental load. The ideas and procedures presented here can form a basis for further work to build ships with an improved safety performance.

The thesis reports on different methods developed to assess the structural response of ships to stranding incidents. The stranding process can be divided into two stages. The first stage begins with the initial contact, includes the sliding phase and ends in the final stranding position. The vessel does not suffer important damage during this stage. The process can be simulated independently using existing kinetic models. In the second stage the vessel is subjected to tidal actions. The structural damage and the integrity of the stranded vessel has to be solved. The second stage is investigated in the present work. During stranding a vessel comes to rest upon a soft seabed and can be subjected to the tide. If the water recedes, the hydrostatic forces change significantly. They depend on the water level and the trim, which result from the new equilibrium position. The maximum shear force is at the same position as the maximal change or sometimes at the maximum of the bending moment. The interaction of the contact force with the hogging bending moment affects the longitudinal resistance of the hull. If the ultimate hull girder strength is reached, global damage occurs.

All of the proposed methods have been exemplarily applied to a Postpanmax container vessel.

First a simple method based on the ideal-elastic beam theory is given for calculating the bending moment, the shear force and the heel angle during stranding, whereby flooding of tanks can be taken into account (see chapter 3 ). The new equilibrium position is determined on the basis of the real ship form. The correct hydrostatic forces, as a function of water level and stranding position, are used to determine the moments and forces due to stranding. The method is not limited to a specific ship type or load case and comparison with existing formulas showed that the method gives reasonable results. The critical combinations of grounding point/area, surface drawdown and load case (with or without flooding) that lead to an exceedance of the class permissible moment and shear force can be calculated.

A combinational optimisation is solved and implemented with a user interface for an easy and accurate representation of the results. All critical combinations can be determined at once even for parameters that lie in between calculated stranding incidents. It is possible to superimpose the critical cases and weight the influence of bending moment versus shear force.

Diverse standing scenarios are calculated with the Postpanmax vessel. Of all calculations stranding incidents amidship result in the highest bending moments and shear forces. The overstepping of the permissible moment and shear force is always close to the contact point. Considering the shear force, almost all positions of grounding are critical for 5 
$\mathrm{m}$ of receding tide. Considering only the bending moment, midship stranding positions are critical. The highest exceedance of permissible moment and shear force occurs for stranding in the bow and stern region and is due to the shear forces.

To get information about local and global damage, the influence of the ground and the point of total collapse of the structure, a simulation procedure based on the finite element method is introduced. Neither the ship nor the ground nor the external forces are simplified. The method is based on true loading conditions that are gained from the simple method presented in chapter 3 . The chosen ship is completely modelled and the ground is simulated with a well-known ground model. For the purpose of stranding simulations the Mohr-Coulomb model is sufficient. The local and global damage of the structure can be examined as a function of the soil characteristics, the ground geometry, the position of stranding and the ebb tide. The simulation method is applicable to all ship types and ground geometries and to many soil characteristics. It is very insusceptible to modifications and is robust. The verification of the ship and ground model shows that reasonable results are achieved.

Most calculated scenarios end in global damage to the structure and a fractured outer hull. The degree of damage, the damage mode and the ultimate strength depend on the ground geometry and the soil characteristics. It is necessary to model the ground with a sand material model because the hull girder is not always less prone to hull collapse if set onto a soft bank.

The midship stranding scenario is the worst-case scenario and the main findings are:

- In all calculated midship scenarios the main transverse fold/fracture in the double bottom and side structure is mainly due to the bending moment, whereas the isotropic buckles in the side structure and the compression of the bilge structure (longitudinal folds) are caused by shear forces.

- In the plastic range it is valid that the longer the contact area is the higher the moments that can be absorbed by the structure.

- During stranding amidship the structure does not reach the class permissible ultimate hull girder strength.

- A rigid ground is not a conservative border for soil characteristics.

- The bending moment curves for different soft ground geometries can be estimated if one simulation is solved. The larger the initial ground plateau and the smaller the slope angle the higher the ultimate strength, whereby the detailed failure mode is different in each case.

Stranding in the bow results in the second highest degree of damage. The damage is almost exclusively caused by the shear forces. For the stern scenario the damage is insignificant. For the bow and stern scenarios the rigid ground is a conservative border. Calculating stranding in the stern section gives only a little more information than the estimation with the simple method, whereas stranding in the bow region results in an 
unexpected early failure of the outer shell plating. The degree of collapse cannot be concluded from the height of the external forces and moments by the simple method (chapter 3).

Furthermore, it is shown that real incidents can be recalculated with very good conformance. The damage caused by the stranding of the Fowairet is reproduced by applying the simulation method.

The bending moments and shear forces have a different influence not only on the damage but also on the hull capacity. A procedure and the resultant equations are given to gain moment-shear force interaction curves. Those curves are based on the true and combined loading conditions during stranding. For any cross section of a ship a curve can be calculated and the influence of the shear force on the ultimate hull girder strength during stranding can be evaluated. The procedure is validated by experiments and compared to the literature.

Different cross sections lead to different moment-shear force interactions. The curves of the cross sections can be ordered starting with the maximum curve (stern, midship and bow) in the same order as their stiffness degrees and their sectional area. With the linear dependency of section area to ultimate strength and of ultimate moment to ultimate shear force a relation between the ultimate values can be found. The proposed equations can be used to gain interaction curves for any cross section whereby only one ultimate value (moment or shear force) is needed. This value should be calculated with FE methods. The formulas from the literature do not give reliable results and the ultimate strength determined with classification rules overestimates the maximum bending moment. Furthermore, the coefficients of an equation already referenced in literature are modified. Thereby different coefficients are needed for the stern section of a container vessel than for the rest of the ship.

The comparison of the simple sections to the equivalent fully modelled cross sections shows that the cut outs decrease the stiffness of the section and thus the ultimate strength. In contrast to the fictive load cases, the true loading of the structure during stranding reduces the ultimate strength by $10 \%$. 



\section{Bibliography}

[1] Alsos, H. S. and Amdahl, J. On the resistance of tanker bottom structures during stranding. Marine Structures, 2007(4):218-237, 2007.

[2] Amdahl, J.; Kavlie, D.; and Johansen, A. Tanker grounding resistance. In H. Kim and J. W. Lee, editors, PRADS 1995, volume 2 of Proc. PRADS 1995, pages 2.10722.1083. 1995. ISBN 89-9500160-7.

[3] American Bureau of Shipping. Guide for dynamic based design and evaluation of bulk carrier structures. 1995 .

[4] Ansys. Release 14.0 Documentation for ANSYS. 2011.

[5] Bleich, F. Buckling strength of metal structures. McGraw-Hill, New York, 1952.

[6] Bureau Veritas. Rules for the classification fo steel ships: Part B, Chapter 6. 2003.

[7] Caldwell, J. B. Ulitmate longitudinal stength. Transactions of RINA, (107):411-430, 1965.

[8] Committee III.1. Discussion of the technical Committee III.1. In P. S. Frieze and R. A. Shenoi, editors, ISSC 2006, volume 3 of Proc. ISSC 2006, pages 117-142. 2006. ISBN 0-85432-855-6.

[9] Committee III.1. Ultimate strength. In P. S. Frieze and R. A. Shenoi, editors, ISSC 2006, volume 1 of Proc. ISSC 2006, pages 353-438. 2006. ISBN 0-85432-855-6.

[10] Committee V.1. Damage assessment after accidental events. In C. D. Jang and S. Y. Hong, editors, ISSC 2009, volume 2, pages 1-72. 2009. ISBN 978-89-954730-1-6.

[11] Committee VI.2. Ultimate hull girder strength. In H. S. Y. Ohtsubo, editor, ISSC 2000, volume 2 of Proc. ISSC 2000, pages 321-391. Elsevier, 2000. ISBN 0-08043602-1.

[12] Det Norske Veritas. Rules for classification of ships: Part 3, Chapter 1. 2009. URL http: / / exchange.dnv.com

[13] DIN 18137-2. Soil, investigation and testing- determination of shear strength. 2011.

[14] Drucker, D. C. The effect of shear on the plastic bending of beams. Journal of Applied Mechanics, 1956(23):509-514, 1956.

[15] Egge, E. D.; Jamaillo, D.; Scharrer, M.; Zhang, L.; and Germanischer Lloyd. Abschlussbericht zum Vorhaben MTK0614, Kollisionsberechnung in schiffbaulichen Entwurfssystemen: Bericht Nr. ESS 2002.183. 2002. 
[16] European Union. Tide times: Tidal river development.

URL www.tide-project.eu

[17] Germanischer Lloyd. POSEIDON- User Manual. 2013.

[18] Germanischer Lloyd. Rules \& guidelines 2013; Chapter 1. 2013.

URL http: / / www.gl-group.com

[19] Gordo, J. M. and Soares, C. G. Experimental evaluation of the ultimate bending moment of a box girder. Marine Systems and Ocean Technology, 1(1):33-46, 2004.

[20] Grabe, J. Bodenmechanik und Grundbau. Hamburg-Harburg, 3. edition, 2004.

[21] Großmann, C. and Terno, J. Numerik der Optimierung. Teubner-Studienbücher Mathematik. Teubner, Stuttgart, 2., durchges. aufl edition, 1997. ISBN 3519120909.

[22] Gudehus, G. Bodenmechanik. Ferdinand Enke Verlag, Stuttgart, 1981. ISBN 9783-432-92181-0.

[23] Hampel, B. Diplomarbeit: Auswertung und Analyse der Schadensfalldaten über Kollisionen und Grundberührungen von Schiffen des Germanischen Lloyd. 1997.

[24] Hugenschmidt, M. Lasermesstechnik: Diagnostik der Kurzzeitphysik. Lasermesstechnik, 2007.

[25] IACS. Common structural rules for bulk carriers. 2006.

URL http: / /www.iacs.org.uk

[26] IACS. Common structural rules for double hull oil tankers. 2006.

URL http: / /www.iacs.org.uk

[27] Janele, T. O. Tragfähigkeit unterschiedlicher Schiffsquerschnitte unter Biege- und Querkraftbelastung. Project Thesis, Hamburg University of Technology, 2013. Supervised by Zipfel, B. and Lehmann, E.

[28] Jungnickel, D. Optimierungsmethoden: Eine Einführung. Springer-Lehrbuch. Springer, Berlin, 1999. ISBN 3-540-66057-7.

[29] Karlsson, U. B.; Ringsberg, J. W.; Johnson, E.; Hoseini, M.; and Ulfvarson, A. Experimental and numerical investigation of bulb impact with a ship side-shell structure. Marine Technology, (Vol. 46 No. 1):16-26, 2009.

[30] Kolymbas, D. Geotechnik: Bodenmechanik, Grundbau und Tunnelbau. SpringerVerlag Berlin Heidelberg, Berlin and Heidelberg, 2007. ISBN 978-3-540-68965-2.

[31] Krüger, S. Dokumentation von e4. Hamburg, 2001.

[32] Krüger, S. Dokumentation von GROUND. Hamburg, 2009.

[33] Kulzep, A. Verhalten von ausgeschäumten Schiffsstrukturen bei Kollisionen und Grundberührungen: Schriftenreihe Schiffbau Bericht 609. Ph.D. thesis, Hamburg University of Technology, Hamburg, 2001. 
[34] Lehmann, E. Grundzüge des Schiffbaus. 2005.

[35] Lehmann, E. Shipbuilding. In H. Meier-Peter and F. Bernhardt, editors, Compendium marine engineering, pages 813-892. Seehafen Verlag, Hamburg, 2009. ISBN 978-3-87743-822-0.

[36] Lehmann, E. and Zhang, L. Nichtlineares Verhalten von ausgesteiften Tragwerken. Springer, Berlin, 1998. ISBN 3-540-63444-4.

[37] Livermore Software Technology Corporation. LS-DYNA theoretical manual. 1998.

[38] Livermore Software Technology Corporation. LS-DYNA keyword user's manual. 2013.

[39] Lloyd's Register. Rules and regulations for the classification of ships. 2005.

[40] Lützen, M. and Simonsen, B. C. Grounding damage to conventional vessels. In World Maritime Technology Conference, pages 1-13. 2003.

[41] MathWorks. R2009b MathWorks Documentation for MATLAB 7.9.0. 2009.

[42] Meier, C. Berechnung von Strukturschäden bei einem Strandungsszenario im Hinterschiffsbereich. Diploma Thesis, Hamburg University of Technology, 2013. Supervised by Zipfel, B. and Lehmann, E.

[43] MSC software. Marc 2008 r1: Volume A-E. 2008.

[44] Naar, H.; Kujala, P.; Simonsen, B. C.; and Ludolphy, H. Comparison of the crashworthiness of various bottom and side structures. Marine Structures, (15):443-460, 2002 .

[45] Nippon Kaiji Kyokai. Rules for the survey and construction of steel ships. 2009.

[46] Nitta, A.; Arai, H.; and Magaino, A. Basis of IACS unified longitudinal strength standard. Marine Structures, (5):1-21, 1992.

[47] Östergaard, C.; Pleß, E.; and Fricke, W. Das Binnenschiff in Flußmündungen. In Jahrbuch der Schiffbautechnischen Gesellschaft, volume 82, pages 134-141. Springer, Berlin, 1988. ISBN 3-540-50431-1.

[48] Paik, J. K. A new concept of the effective shear modulus for a plate buckled in shear. Journal of Ship Research, 39(1):70-75, 1995.

[49] Paik, J. K. and Lee, J. M. An empirical formulation for predicting the ultimate compressive strength of plates and stiffened plates: Joint PNU-SHI Project. Pusan and Korea, 1995.

[50] Paik, J. K. and Mansour, A. E. A simple formulation for predicting the ultimate strength of ships. Marine Science and Technology, (1):53-62, 1995. 
[51] Paik, J. K.; Thayamballi, D. K.; and Che, J. S. Ultimate strength of ship hulls under combined vertical bending, horizontal bending and shearing forces. Transactions of SNAME, (104):31-59, 1996.

[52] Pedersen, P. T. Ship grounding and hull girder strength. Marine Structures, 1994(7):1-29, 1994.

[53] Pedersen, P. T. and Sterndorff, M. J. Grounding experiments on soft bottoms. Journal of Marine Science an Technology, (1):174-181, 1996.

[54] Peschmann, J. Berechnung der Energieabsorption der Stahlstruktur von Schiffen bei Kollisionen und Grundberührungen: Schriftenreihe Schiffbau Bericht 613. Ph.D. thesis, Hamburg University of Technology, Hamburg, 2001.

[55] Peschmann, J.; Kulzep, A.; and Lehmann, E. Grundberührung von Schiffen. In Jahrbuch der Schiffbautechnischen Gesellschaft, volume 91. Springer, Berlin, 1997. ISBN 3-540-65179-9.

[56] Petersen, C. Stahlbau: Grundlagen der Berechnung und baulichen Ausbildung von Stahlbauten. Braunschweig, 2. edition, 1990.

[57] Radaj, D. Schweissprozesssimulation: Grundlagen und Anwendungen. Verl. für Schweissen und Verwandte Verfahren, DVS-Verl., Düsseldorf, 1999. ISBN 9783871551888 .

[58] Reckling, K.-A. Plastizitätstheorie und ihre Anwendung auf Festigkeitsprobleme. Springer, Berlin, 1967.

[59] Reckling, K.-A.; Dannenberg, L.; and Immenköter, K. Die experimentelle Ermittlung der Tragfähigkeit längsversteifter Kastenträger unter reiner Biegebeanspruchung. Schiff und Hafen, 1984(3), 1984.

[60] Reich, M. and Röhr, U. Strukturkollaps von Doppelhüllenkonstruktionen unter Extrembelastungen. In Jahrbuch der Schiffbautechnischen Gesellschaft, volume 94, pages 305-318. Schifffahrts-Verl. Hansa Schroedter, Hamburg, 2000. ISBN 3-54043417-8.

[61] Reich, M. and Röhr, U. BMBF-Vorhaben Fertigungsgerechte Gestaltung von Doppelhüllen: Bauteilfestigkeit. 2001.

[62] Reich, M. and Röhr, U. A hybrid computational model for ship grounding. Schiffstechnik, (52):115-123, 2005.

[63] Reich, M. and Röhr, U. Zur Dynamik der Strandung von Schiffen-Bewegungsgleichungen und Rechenmodell. 2005.

[64] Rutherford, S. and Caldwell, J. B. Ultimate longitudinal strength of ships: a case study. Transactions of SNAME, (98):14.1-14.26, 1990. 
[65] Samuelides, M. S.; Voudouris G.; Toulios, M.; Amdahl, J.; and Dow, R. Simulation of the behaviour of double bottoms subjected to grounding actions. In E. Lehmann and F. Biehl, editors, ICCGS 2007, Proc. ICCGS 2007, pages 93-102. 2007. ISBN 978-3-89220-639-2.

[66] Schenke, S. Optimierung einer Methode zur Vorhersage von Kräften und Momenten bei Grundberührung. Bachelor Thesis, Hamburg University of Technology, 2012. Supervised by Zipfel, B. and Lehmann, E.

[67] Schneider, K.-J.; Goris, A.; and Berner, K. Bautabellen für Ingenieure: Mit Berechnungshinweisen und Beispielen. Werner, Neuwied, 17. edition, 2006. ISBN 3-80415228-7.

[68] Schultz, H.-G. Buckling and post buckling behavior of a transversely stiffened ship hull model. Journal of Ship Research, 1964:6-21, 1964.

[69] Simonsen, B. C. Mechanics of ship grounding. Ph.D. thesis, Technical University of Denmark, 1997.

[70] Simonsen, B. C. and Pedersen, P. T. Analysis of ship groundings on soft sea beds. In H. Kim and J. W. Lee, editors, PRADS 1995, volume 2 of Proc. PRADS 1995, pages 2.1096-2.1109. 1995. ISBN 89-9500160-7.

[71] Simonsen, B. C. and Pedersen, P. T. Dynamics of ships running aground. Journal of Marine Science an Technology, 1995(1):37-45, 1995.

[72] Simonsen, B. C.; Törnqvist, R.; and Lützen, M. A simplified grounding damage prediction method and its application in modern damage stability requirements. Marine Structures, (22):62-83, 2009.

[73] Simonsen, B. C. and Wierzbicki, T. Grounding bottom damage and ship motion over a rock. International Journal of Offshore and Polar Engineering, (Vol. 6 No. 1):195-202, 1996.

[74] Smith, C. Influence of local compressive failure on ultimate longitudinal strength of a ship's hull. In PRADS 1977, pages 73-79. 1977.

[75] Söding, H. Schwimmfähigkeit und Stabilität von Schiffen. Hannover, 1975.

[76] Steimer, K. N. Berechnung von Strukturschäden bei einem Strandungsszenario im Vorschiffsbereich. Bachelor Thesis, Hamburg University of Technology, 2013. Supervised by Zipfel, B. and Lehmann, E.

[77] United Nations. Review of maritime transport 2011. 2011.

[78] van Rijn, L. C. Tidal phenomena in the Scheldt Estuary: Project Nr. 1202016-000. 2010.

[79] Wang, G. Longitudinal strength of ships with accidental damage. Marine Structures, 2002(15):119-138, 2002. 
[80] Wang, G.; Arita, K.; and Liu, D. Behavior of double hull in a variety of stranding or collision scenarios. Marine Structures, (13):147-187, 2000.

[81] Wang, G.; Spencer, J.; and Chen, Y. Assessment of a ship's performance in accidents. Marine Structures, (15), 2002.

[82] Windels, R. Traglasten von Balkenquerschnitten bei Angriff von Biegemoment, Längs- und Querkraft. Stahlbau, 1970(39):10-16, 1970.

[83] Windels, R. Der Querkrafteinfluß bei plastischer Balkenbiegung. Stahlbau, 1947(37):82-85, 1974.

[84] Yao, T.; Imayasu, E.; Maeno, Y.; and Y., F. Influence of warping due to vertical shear force on ultimate hull girder strength. In H. Keil and E. Lehmann, editors, PRADS 2004, volume 1 of Proc. PRADS 2004, pages 322-328. Seehafen Verlag, 2004. ISBN 3-87743-807-5.

[85] Zhang, A. and Suzuki, K. Dynamic FE simulations of the effect of selected parameters. Journal of Ships and Offshore Structures, (1):117-125, 2006. 


\section{A. Parameter Study}

\section{A.1. Optimisation method}

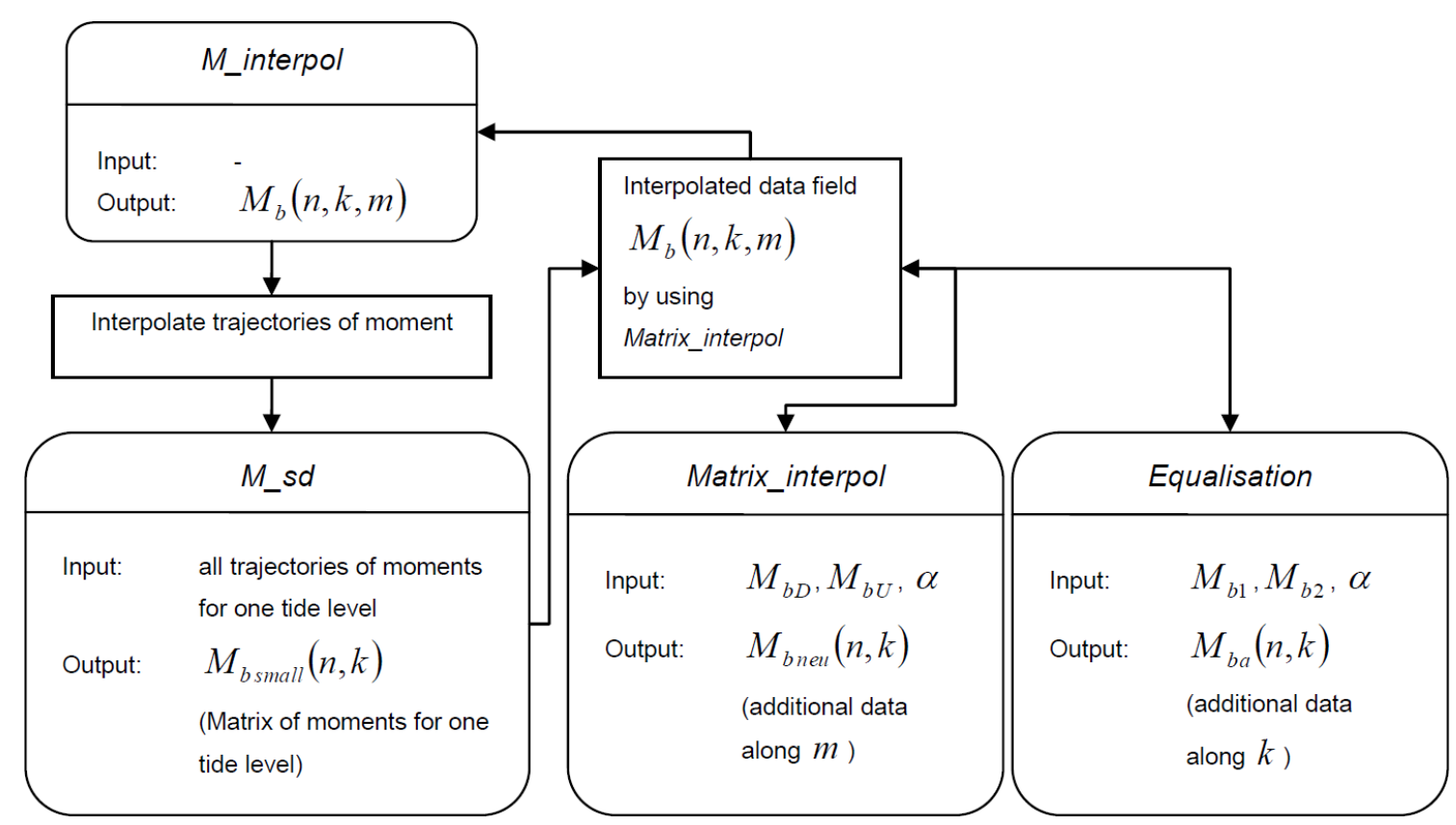

Figure A.1.: Flow chart of function $M_{-}$interpol.

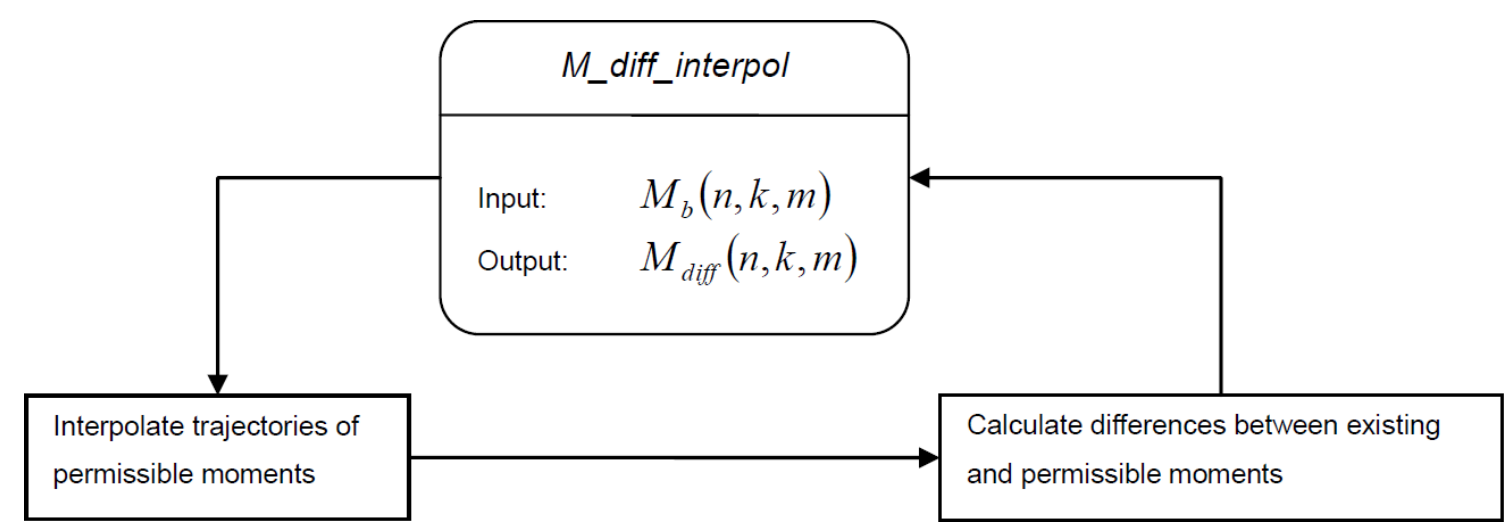

Figure A.2.: Flow chart of function $M \_$diff_interpol. 


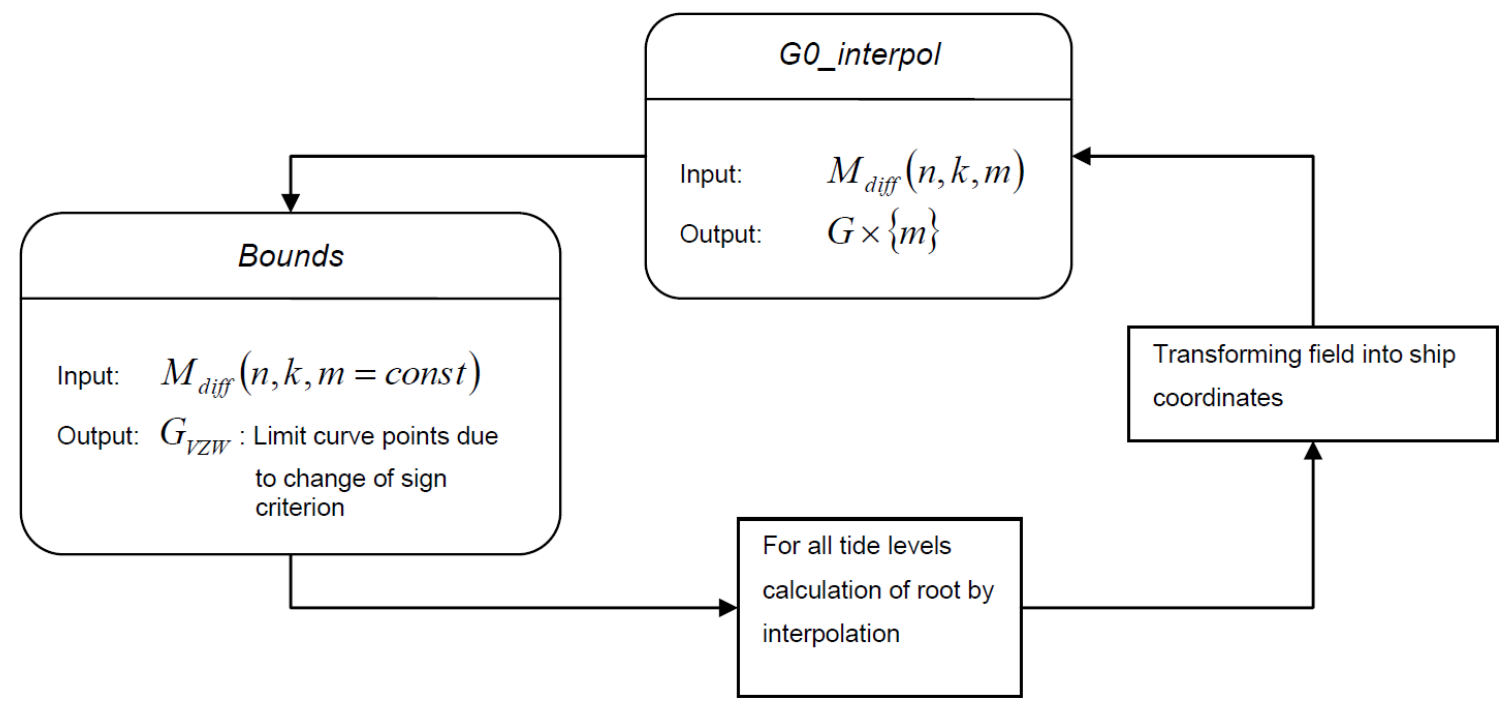

Figure A.3.: Flow chart of function G0_interpol.

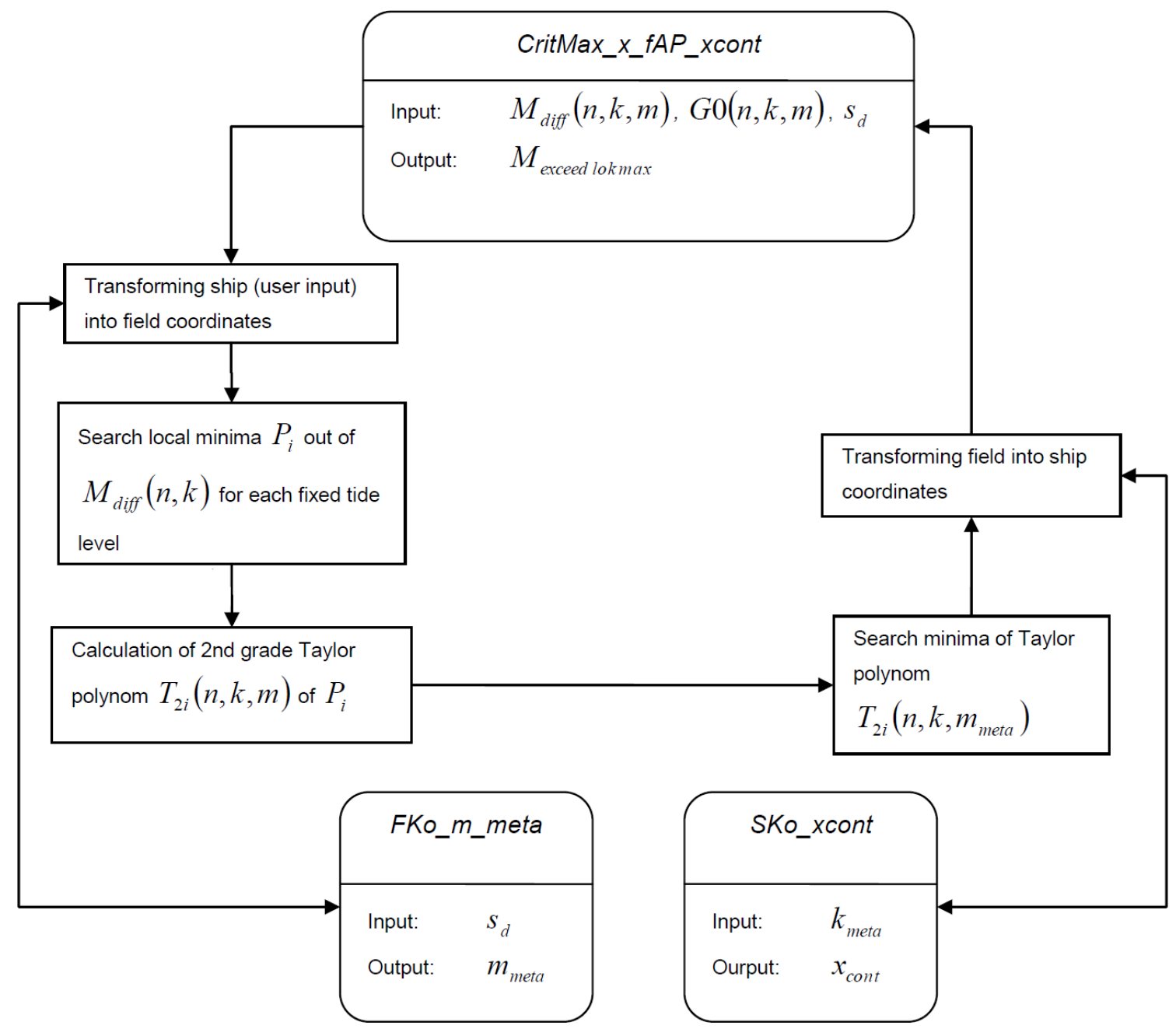

Figure A.4.: Flow chart of function CritMax_x_fAP_xcont. 


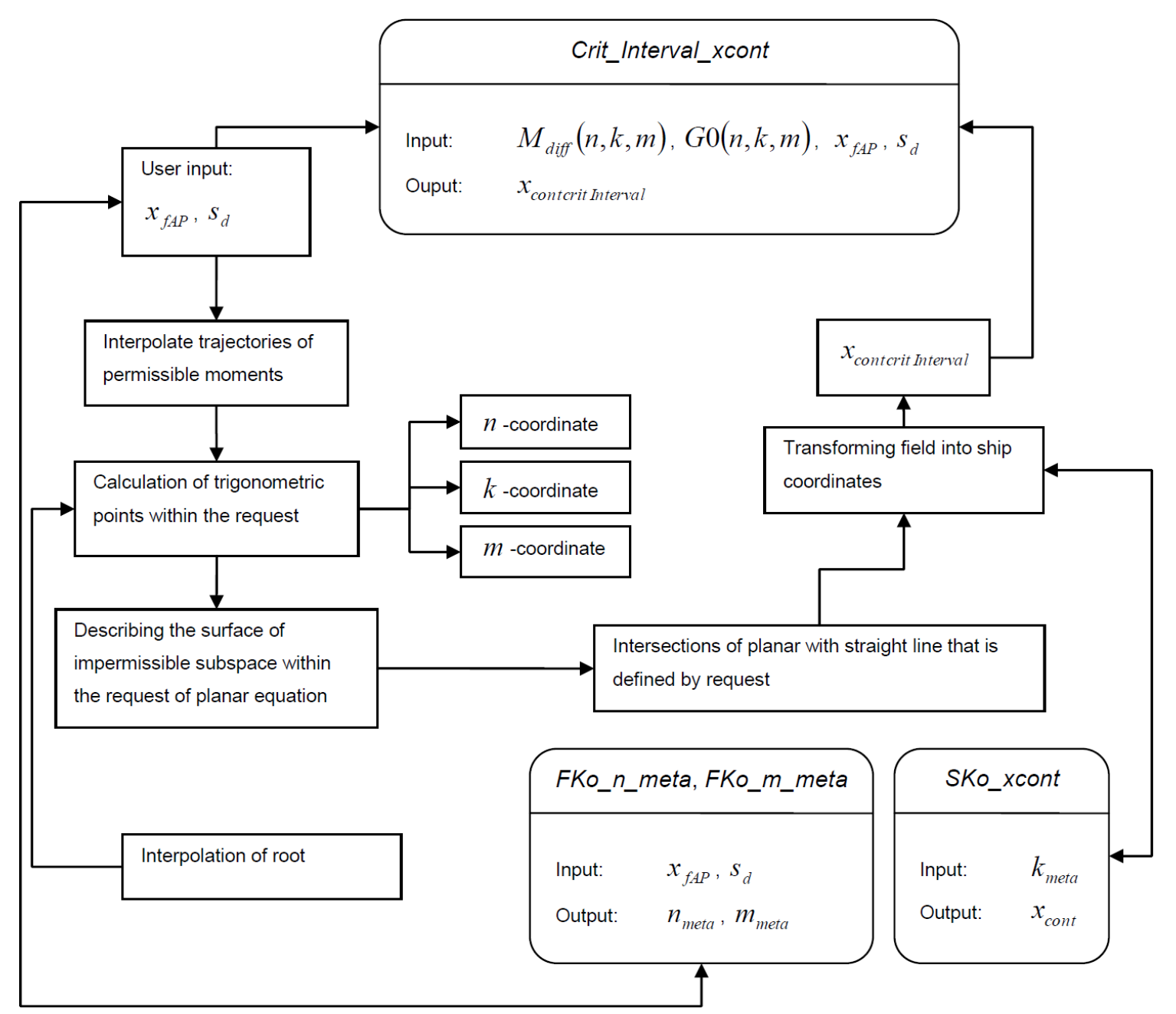

Figure A.5.: Flow chart of function Crit_Interval_xcont. 


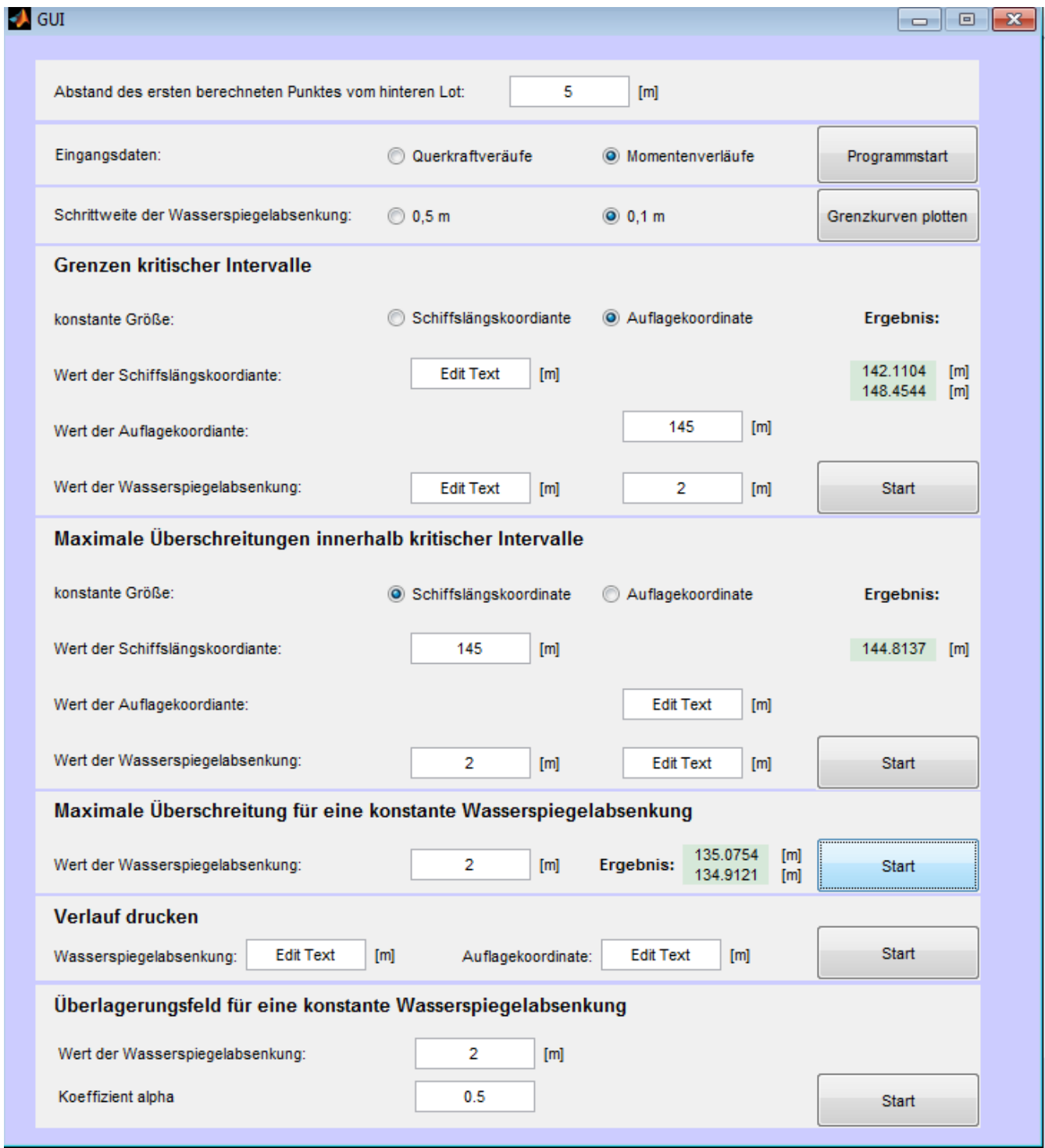

Figure A.6.: GUI for optimisation method. 


\section{A.2. Stranding case B1 and C1}

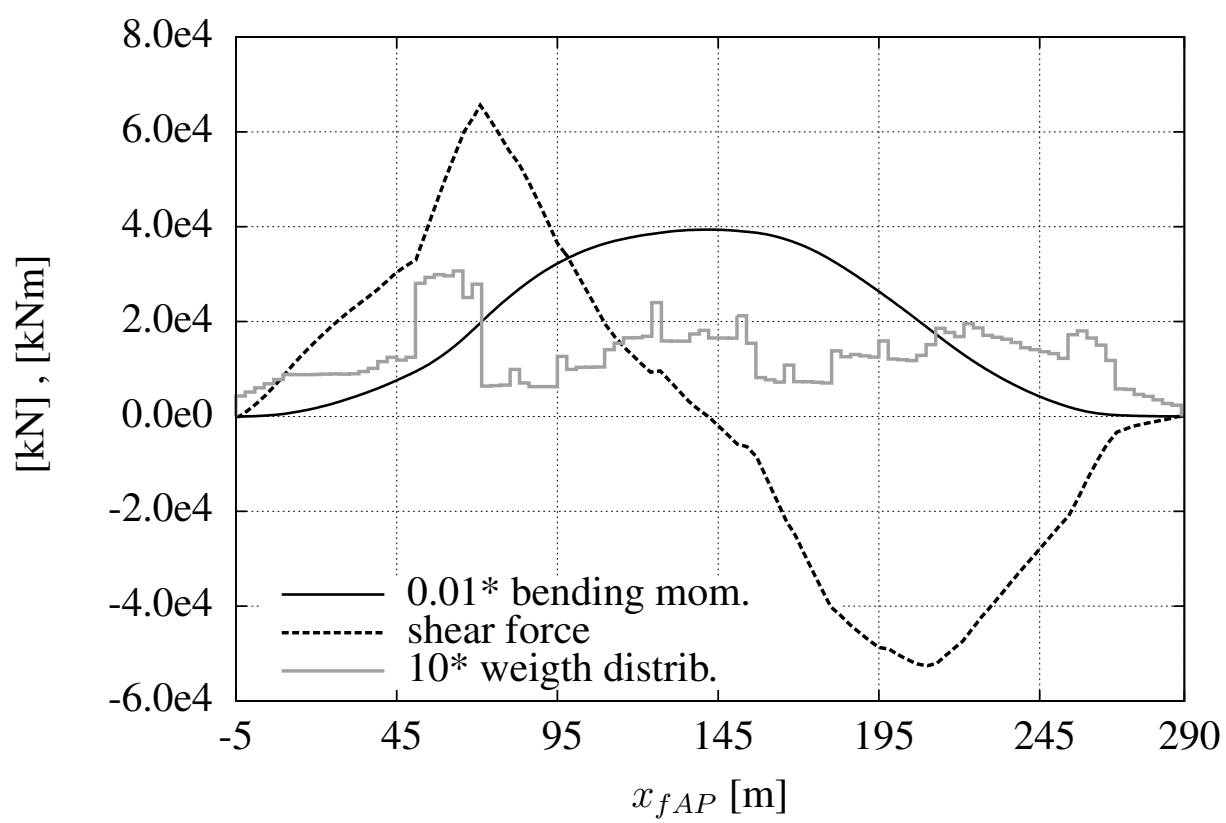

Figure A.7.: Load case B: weight distribution, shear force and still water bending moment.

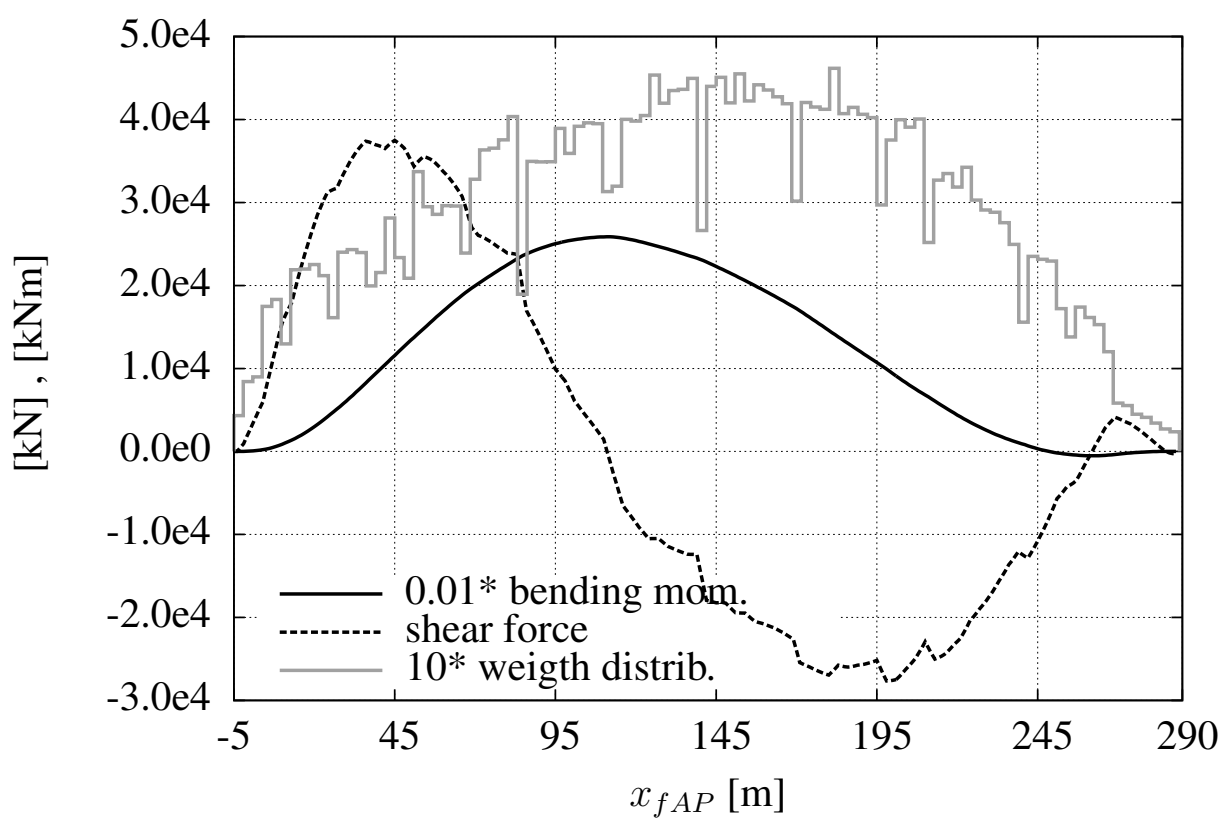

Figure A.8.: Load case C: weight distribution, shear force and still water bending moment. 

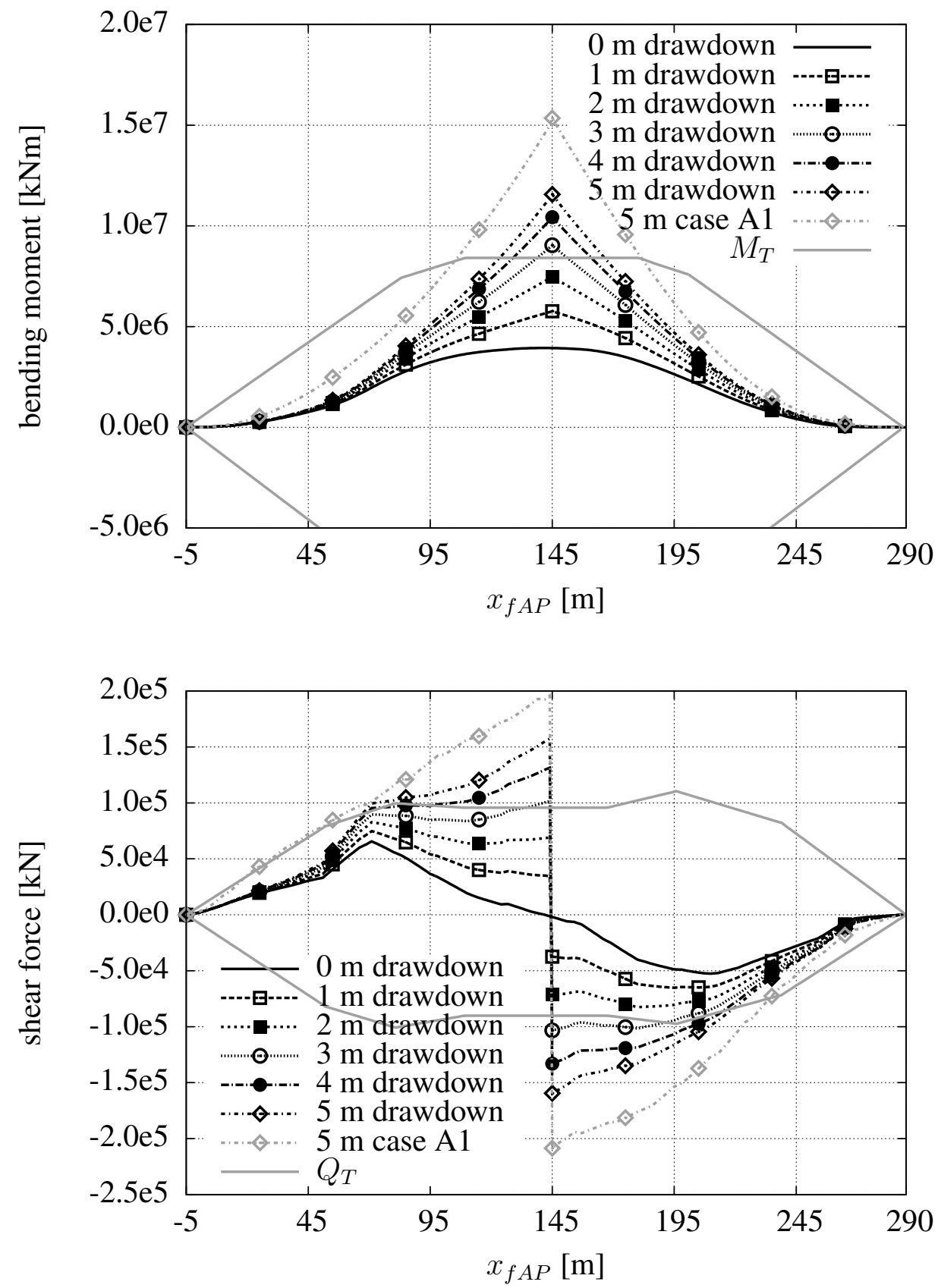

Figure A.9.: Case B1: bending moments and shear forces due to stranding at $145 \mathrm{~m} \mathrm{AP.}$ 

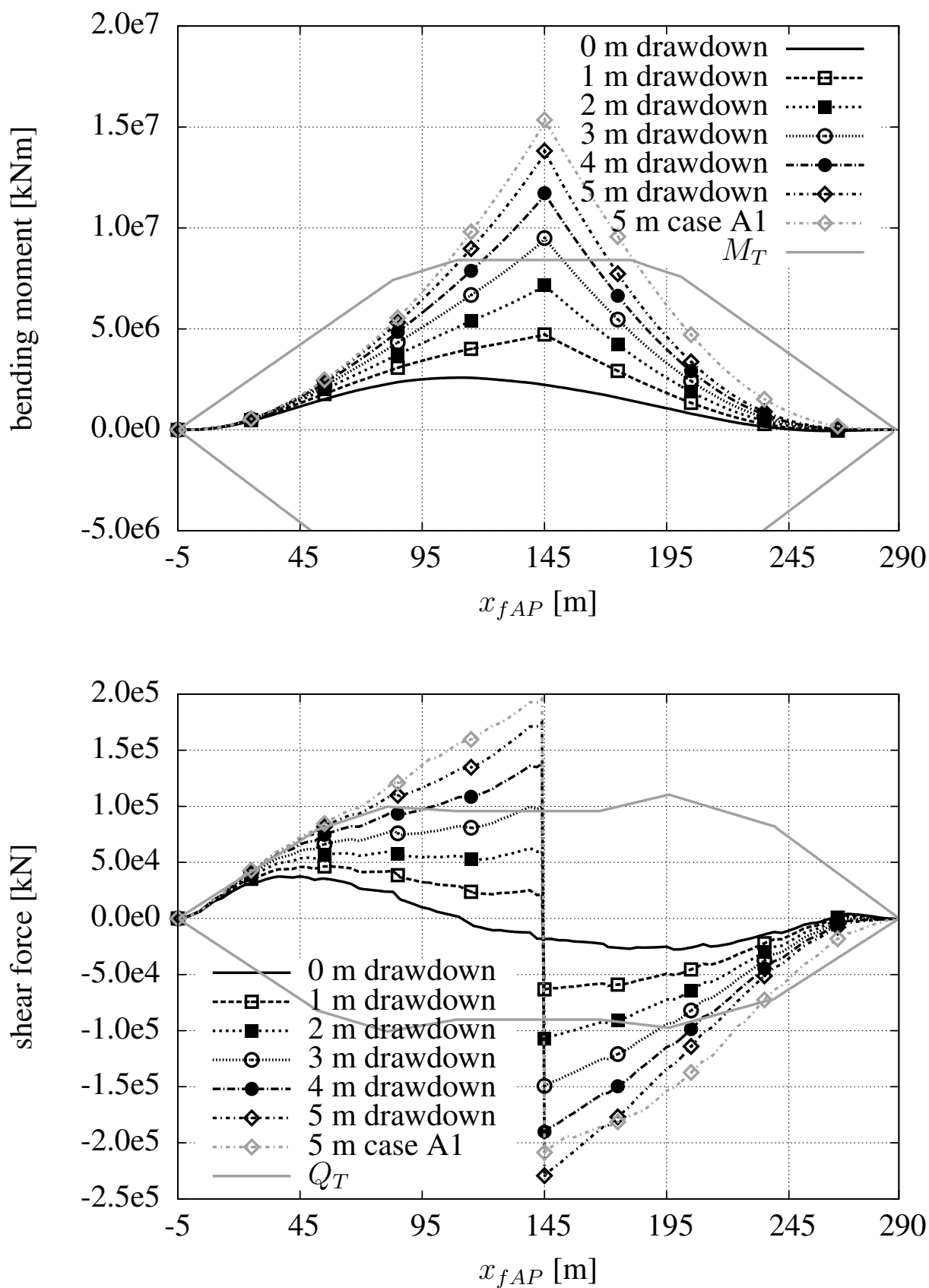

Figure A.10.: Case C1: bending moments and shear forces due to stranding at $145 \mathrm{~m} \mathrm{AP.}$ 


\section{A.3. Stranding case A1f}

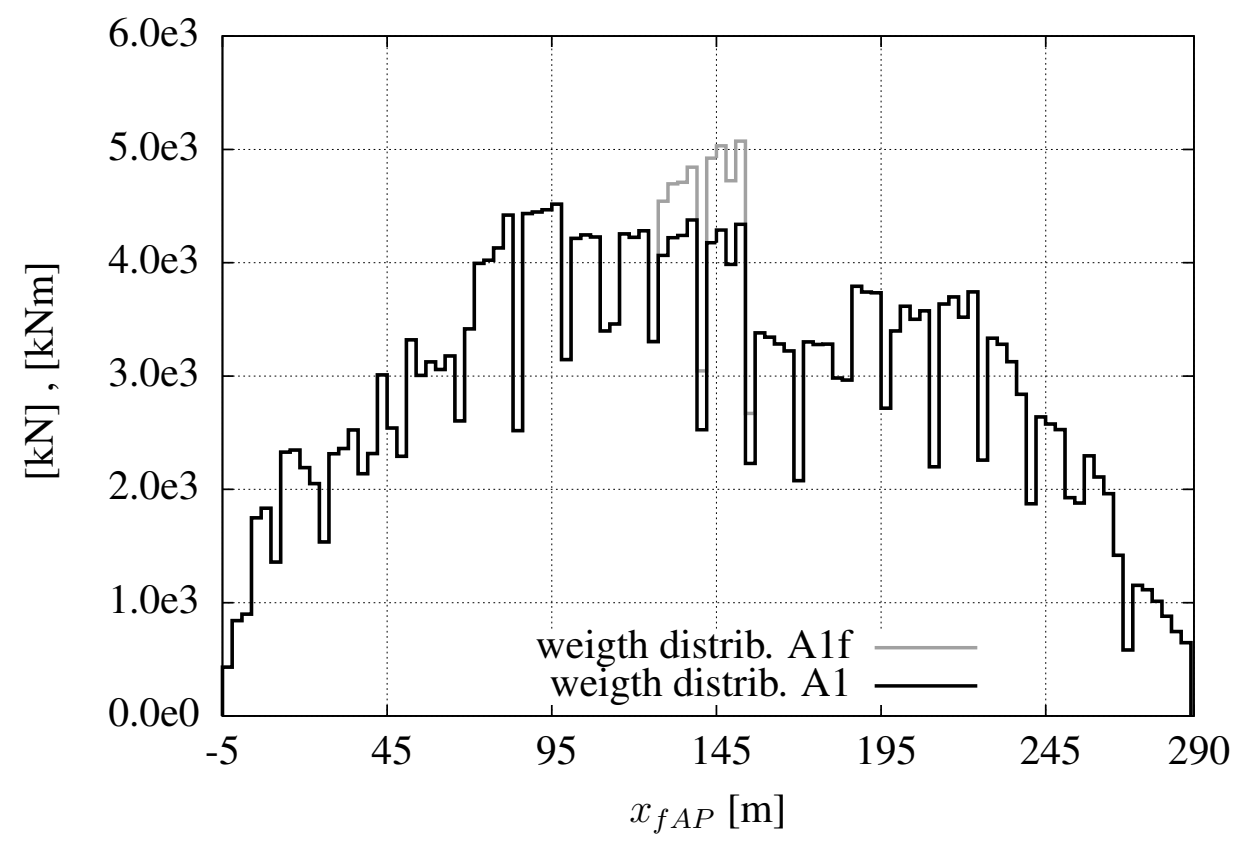

Figure A.11.: Case A1 vs. A1f: weight distribution. 


\section{B. Simulation of stranding scenarios}

\section{B.1. Moment of inertia}

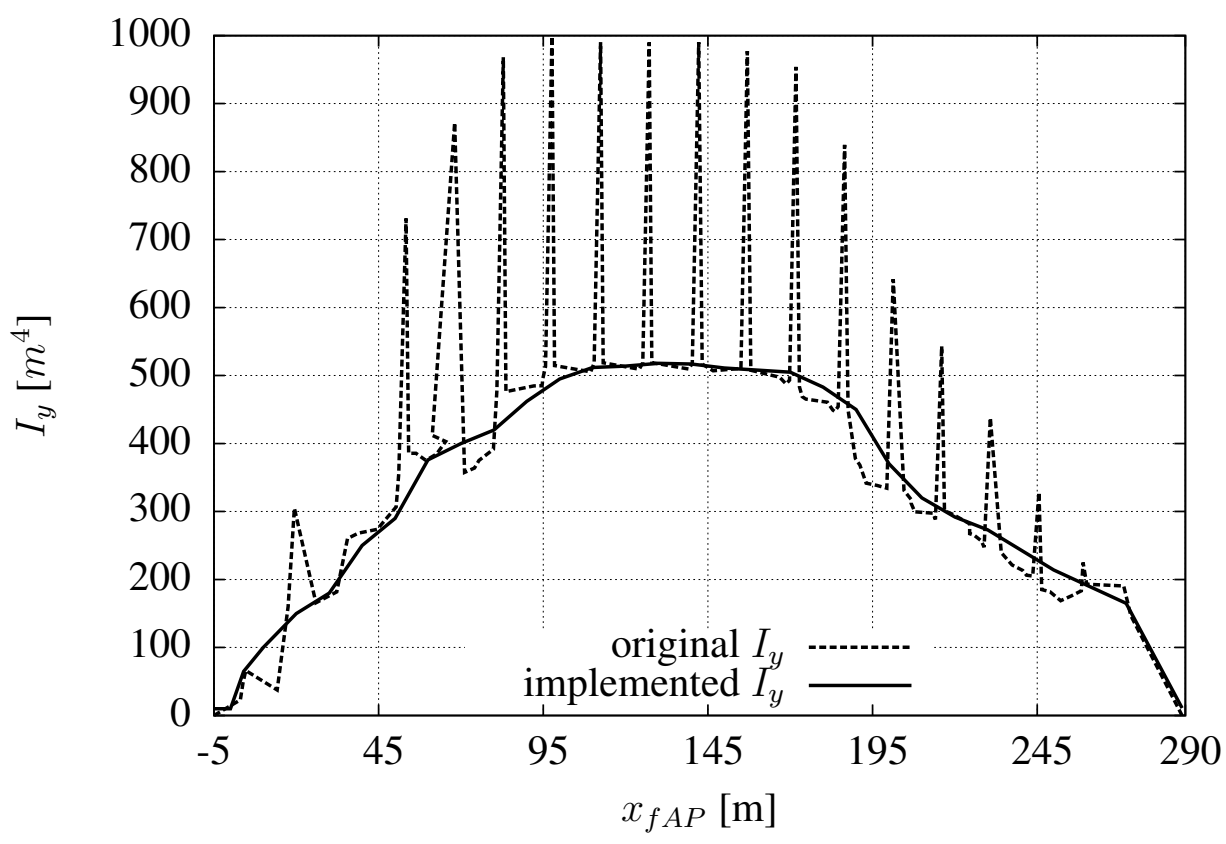

Figure B.1.: Moment of inertia $I_{y}$ of the Panmax and FE model. 


\section{B.2. Construction drawings}

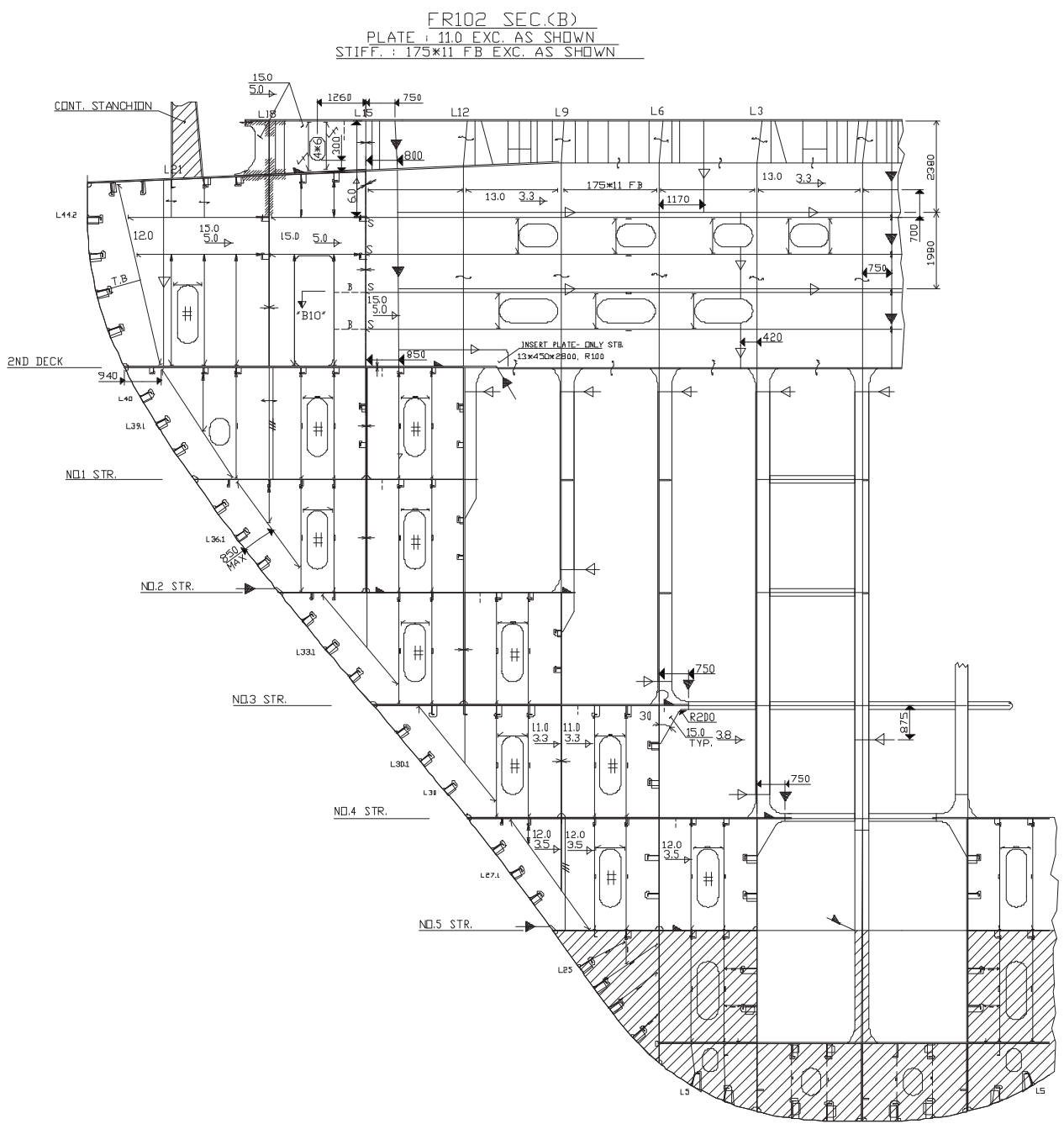

Figure B.2.: Construction drawing of bow section. 


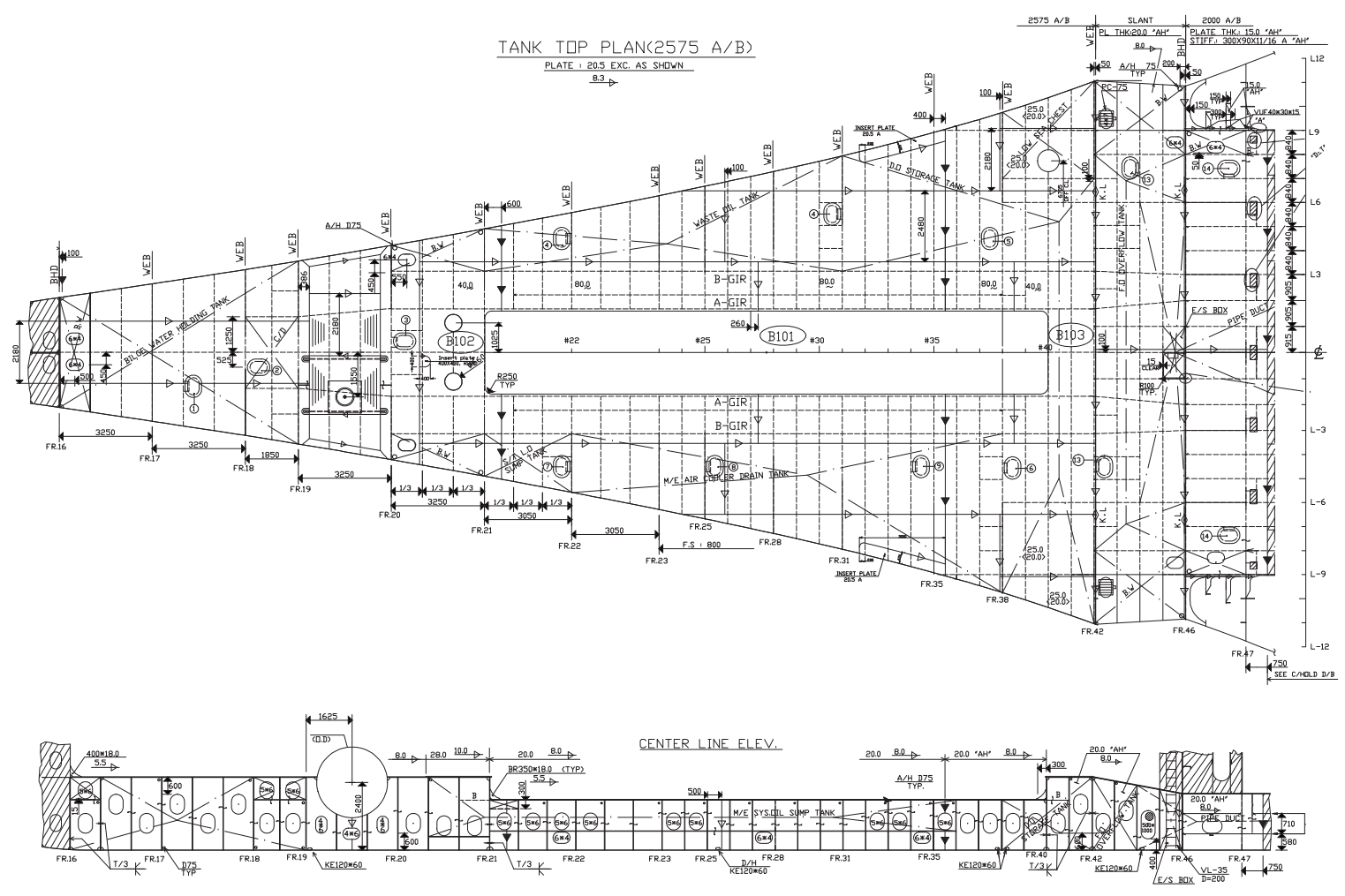

Figure B.3.: Construction drawing of machinery room.

\section{B.3. Oedometer test}

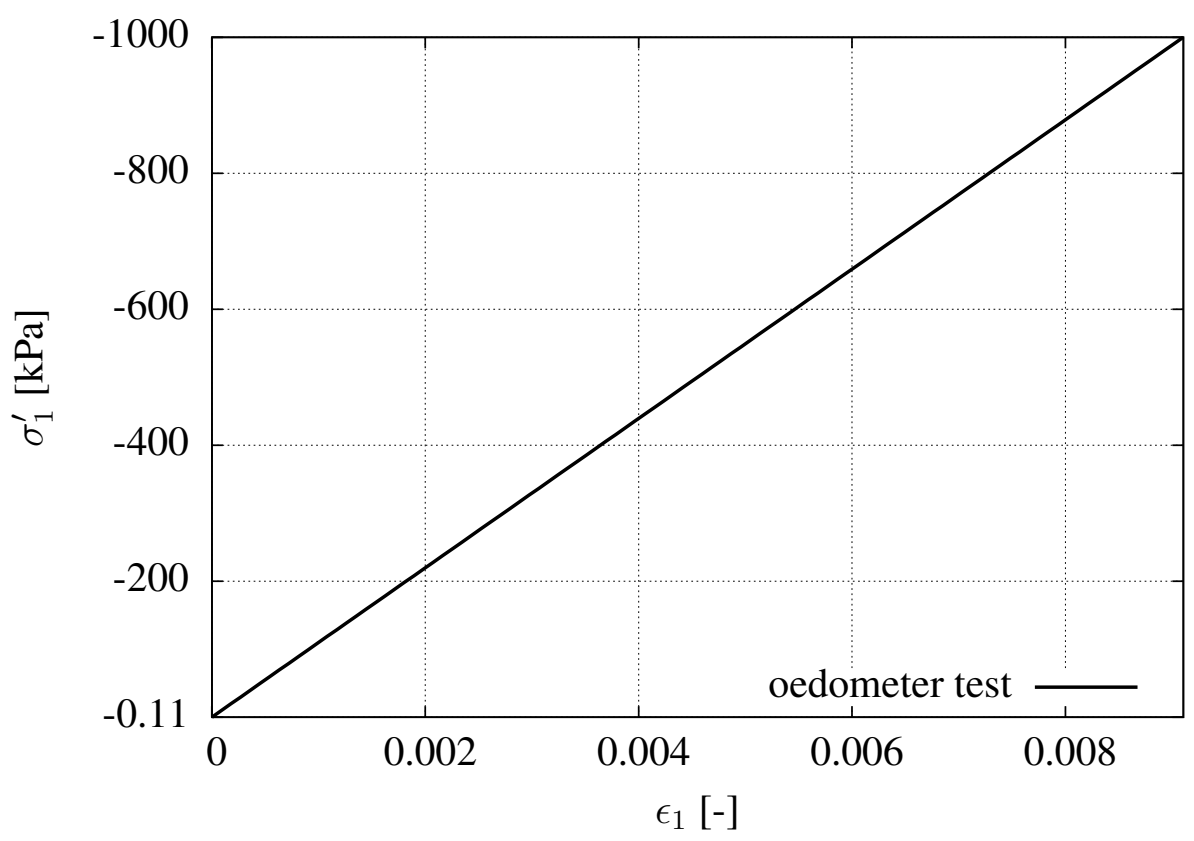

Figure B.4.: Stress-strain diagram of oedometer test. 


\section{B.4. Shear force of Ha7p2 and Sa7p2 at 146, 147 and $148 \mathrm{~m} \mathrm{AP}$}

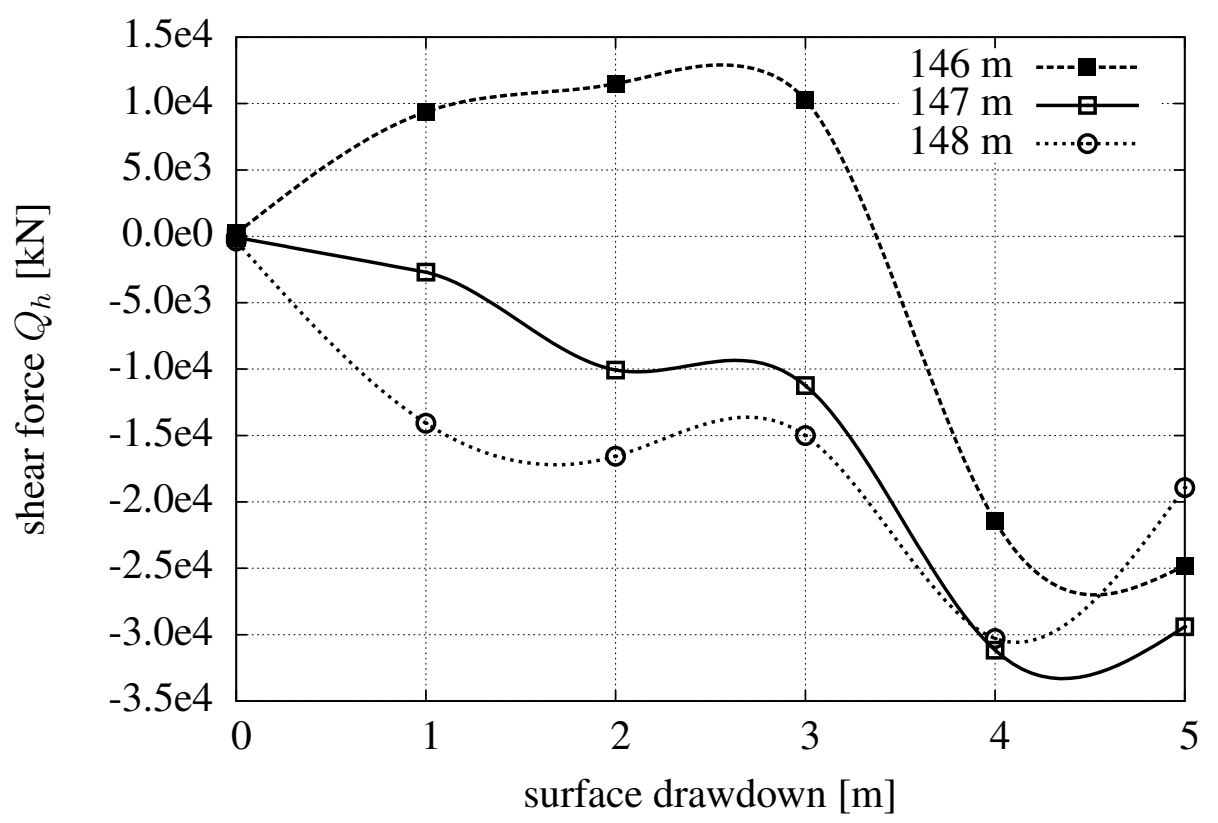

Figure B.5.: Sa7p2: shear force $Q_{h}$ at 146, 147 and $148 \mathrm{~m}$.

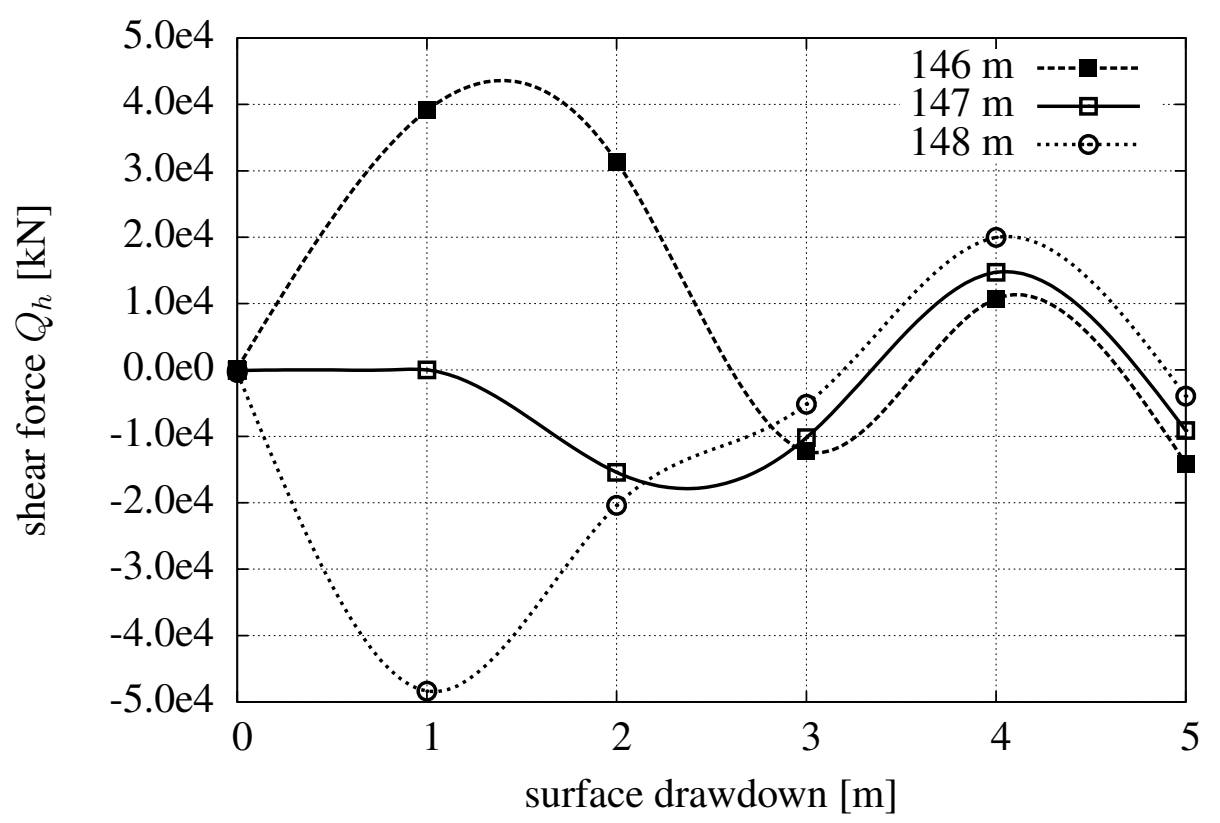

Figure B.6.: Ha7p2: shear force at 146, 147 and $148 \mathrm{~m}$. 


\section{B.5. Influence of ground}

\section{B.5.1. Ground parameters}

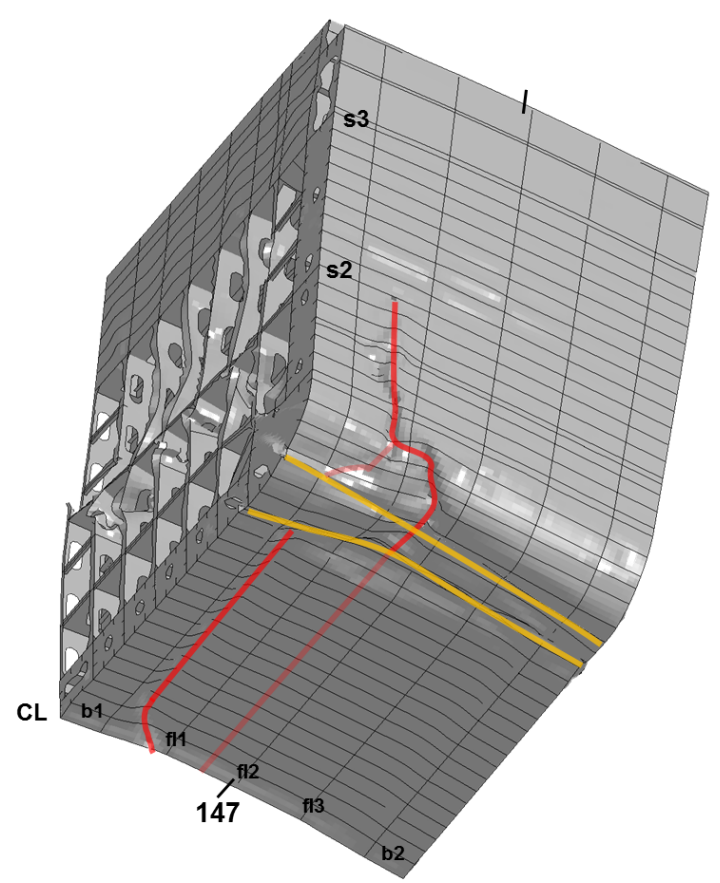

Figure B.7.: SGa7p2: deformation at $5 \mathrm{~m}$ of surface drawdown.

\section{B.5.2. Ground geometries}

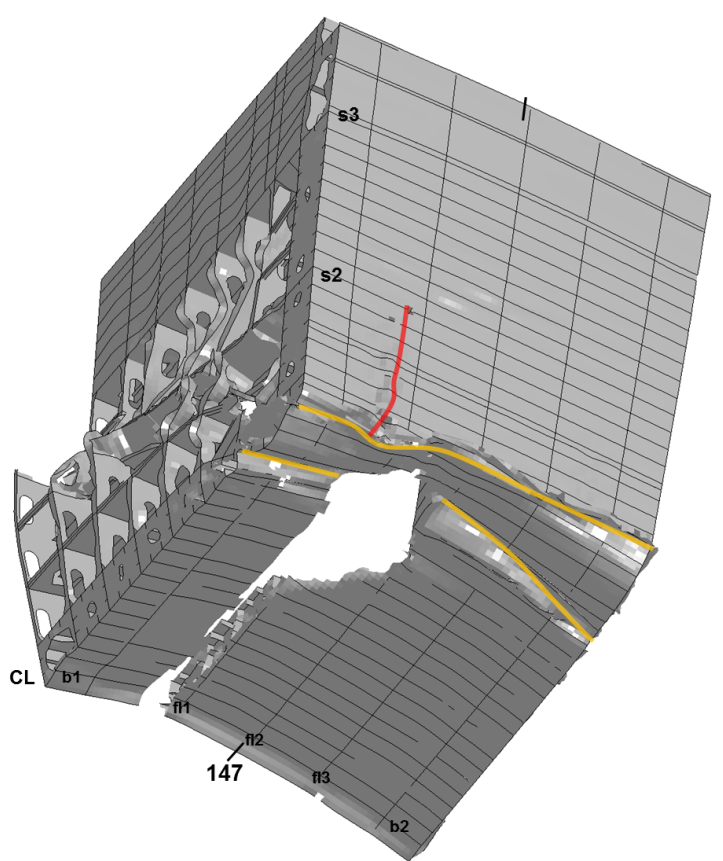

Figure B.8.: Sa15p2: deformation at $5 \mathrm{~m}$ of surface drawdown. 


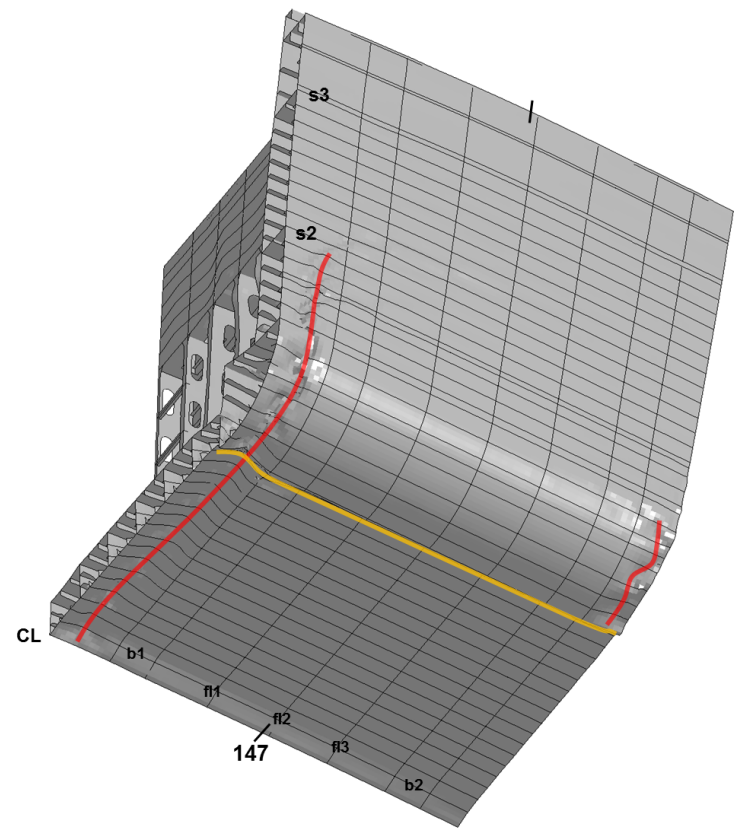

Figure B.9.: Sa7p22: deformation at $5 \mathrm{~m}$ of surface drawdown.

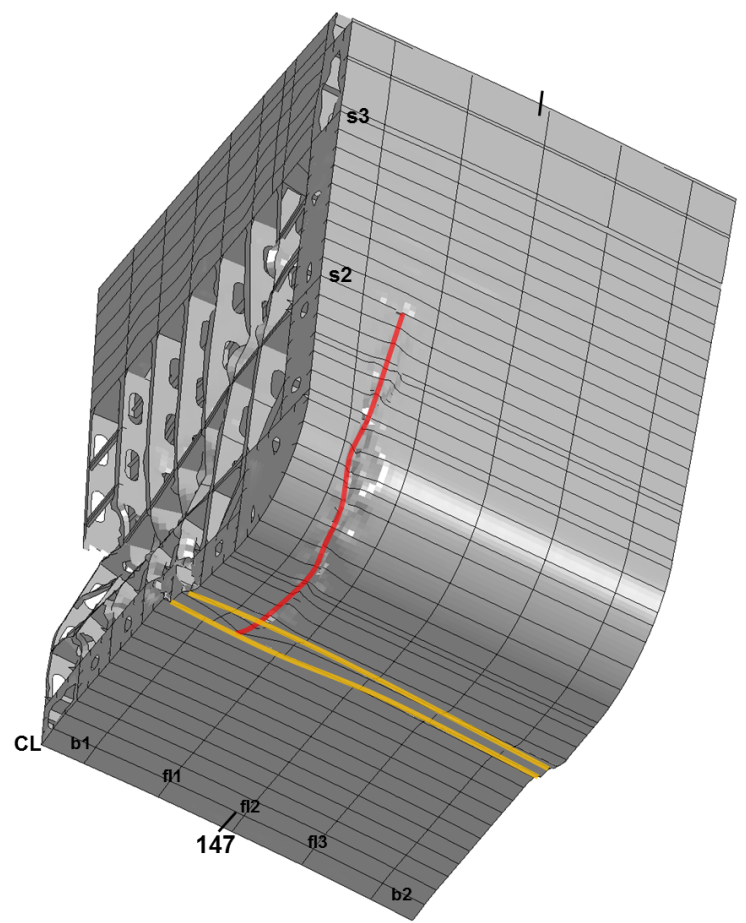

Figure B.10.: Sa7p22w10: deformation at $5 \mathrm{~m}$ of surface drawdown. 


\section{B.6. Results of bow and stern scenarios}

\section{B.6.1. Bow}

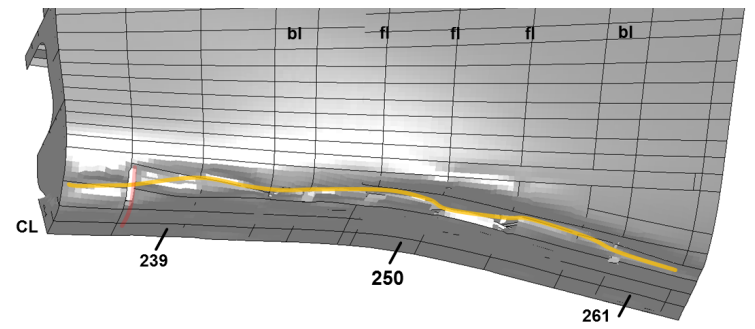

Figure B.11.: Ha7p2 bow: deformation at $5 \mathrm{~m}$ of surface drawdown.

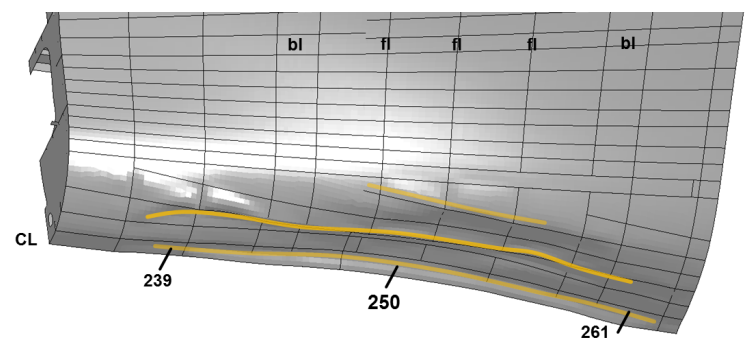

Figure B.12.: Sa7p2 bow: deformation at $5 \mathrm{~m}$ of surface drawdown.

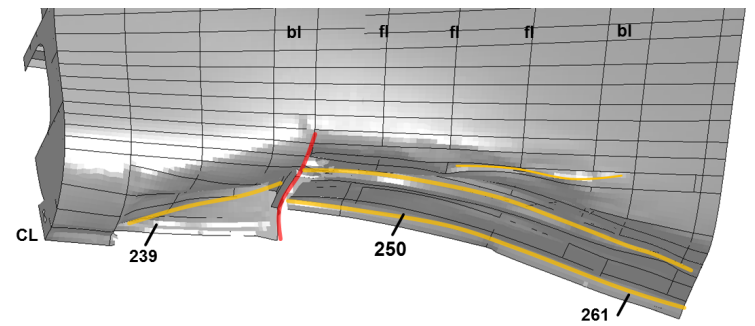

Figure B.13.: Sa15p2 bow: deformation at $5 \mathrm{~m}$ of surface drawdown. 


\section{B.6.2. Stern}

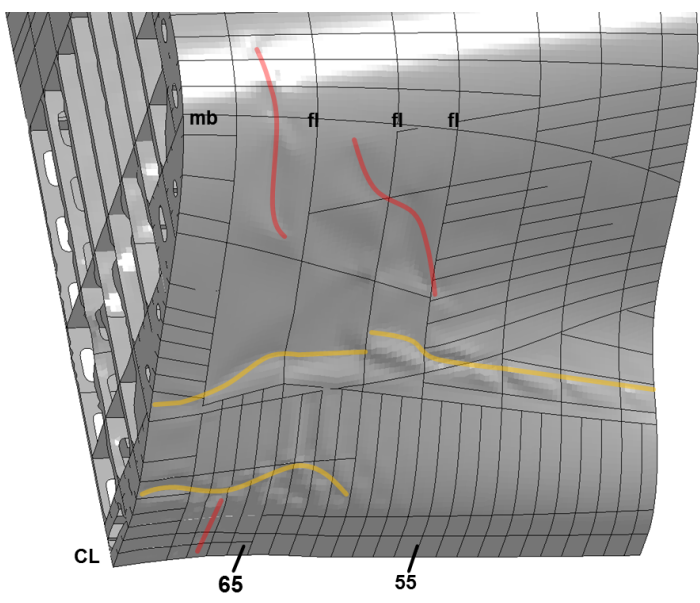

Figure B.14.: Ha7p2 stern: deformation at $5 \mathrm{~m}$ of surface drawdown.

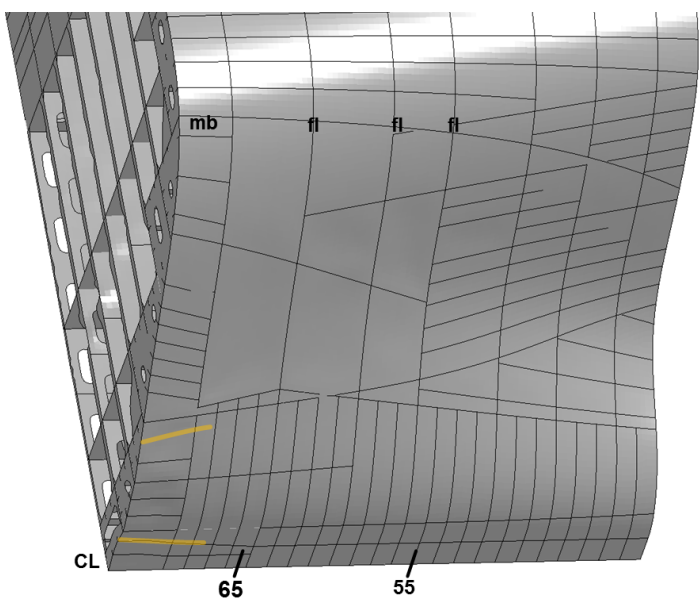

Figure B.15.: Sa7p2 stern: deformation at $5 \mathrm{~m}$ of surface drawdown.

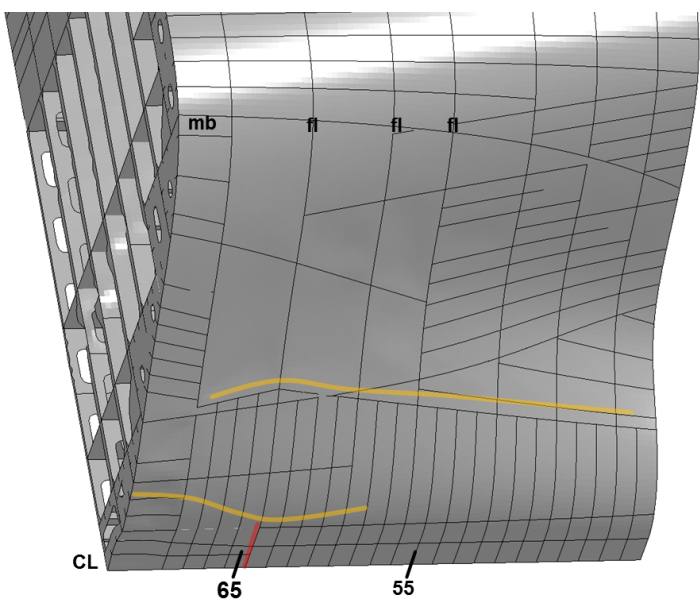

Figure B.16.: Sa15p2 stern: deformation at $5 \mathrm{~m}$ of surface drawdown. 


\section{Ultimate load calculation}

\section{C.1. Cross sections}

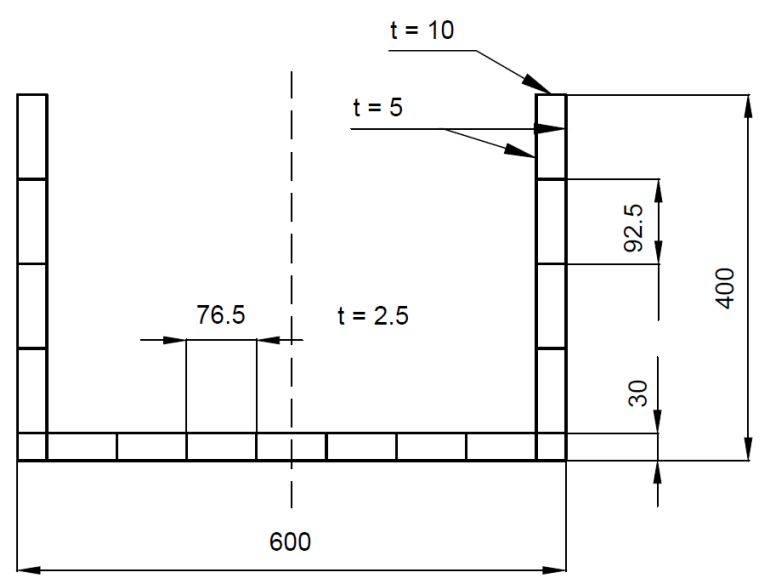

Figure C.1.: Cross section of open box girder.
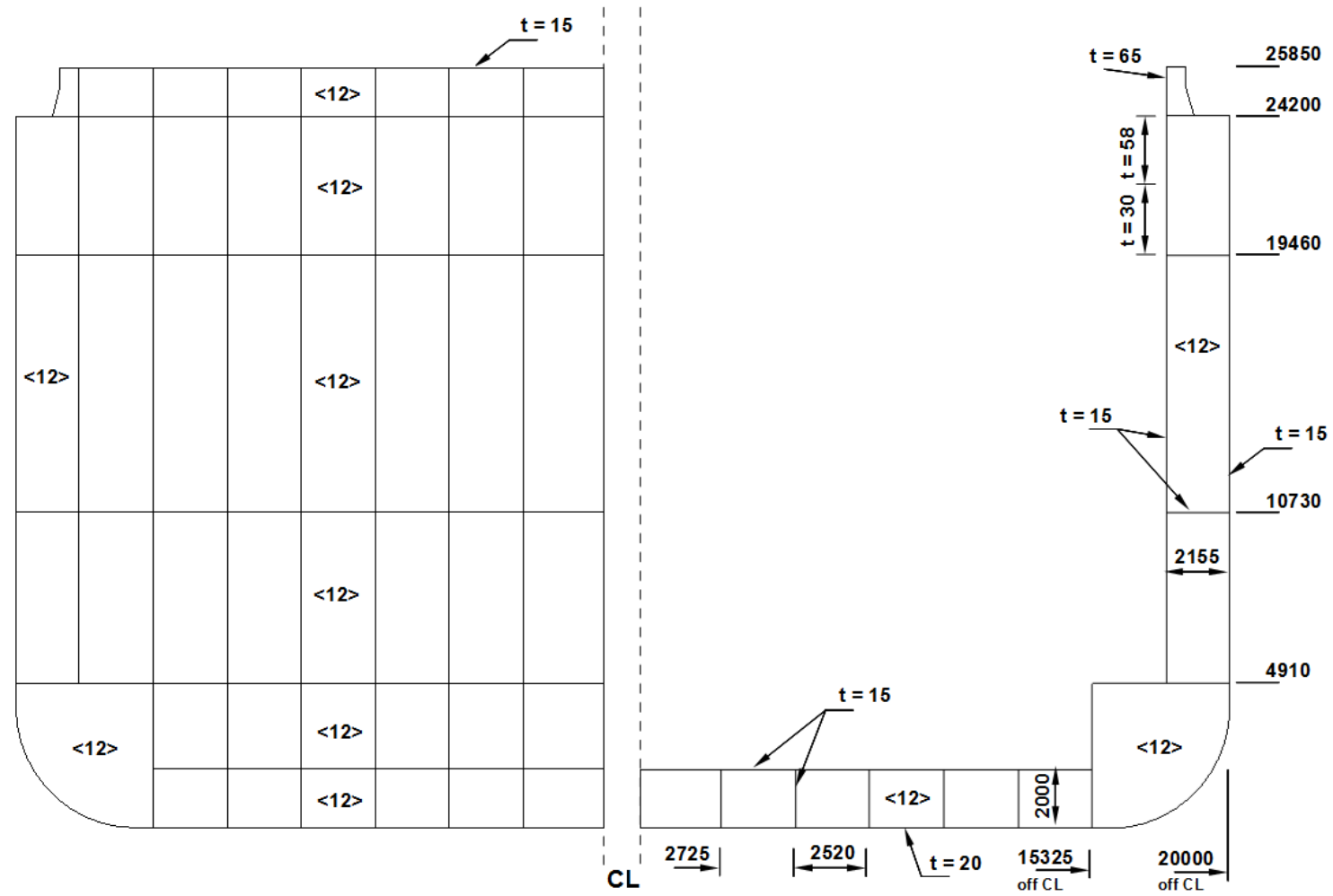

Figure C.2.: Cross section of simplified midship section. 


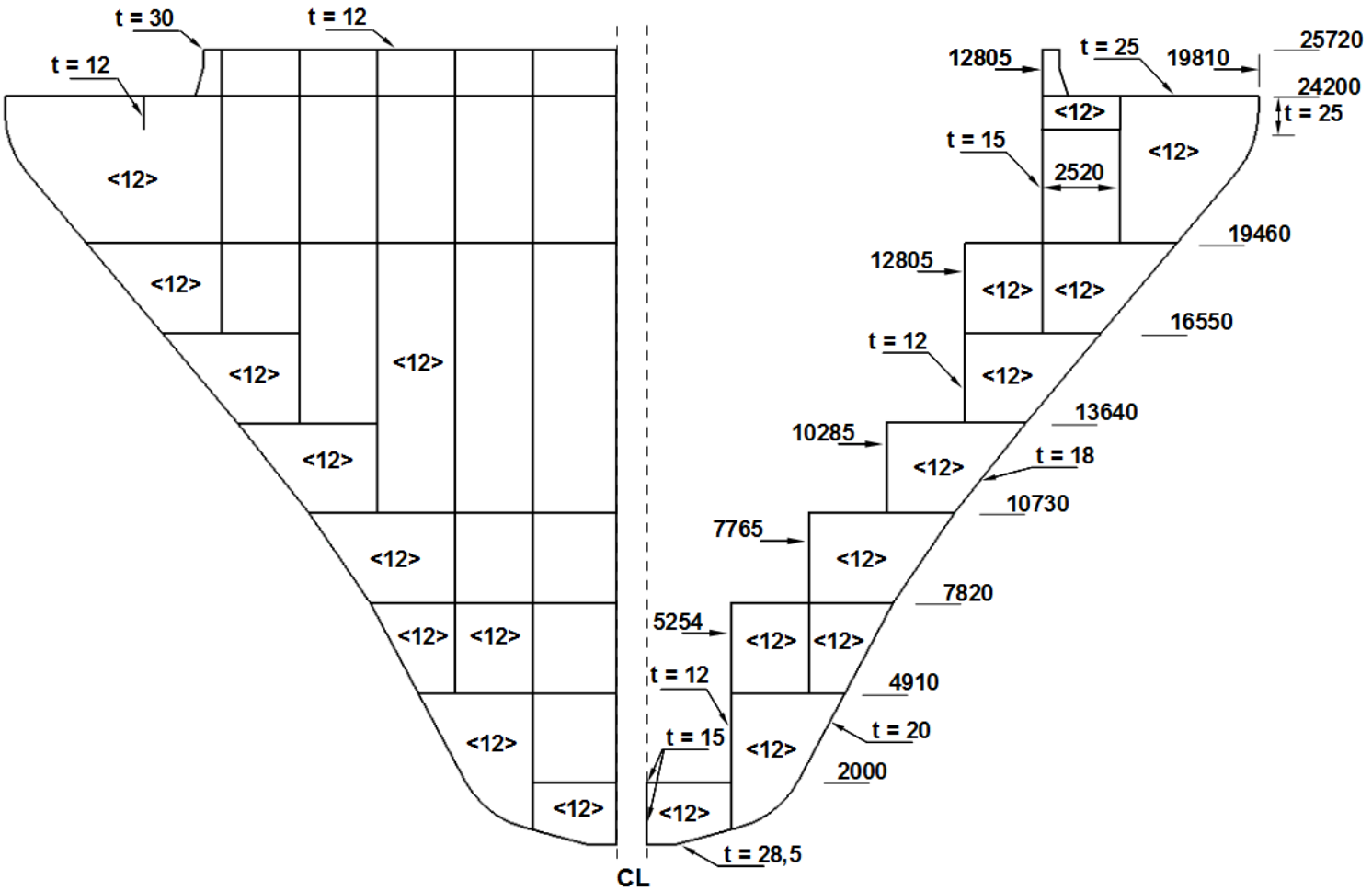

Figure C.3.: Cross section of simplified bow section.

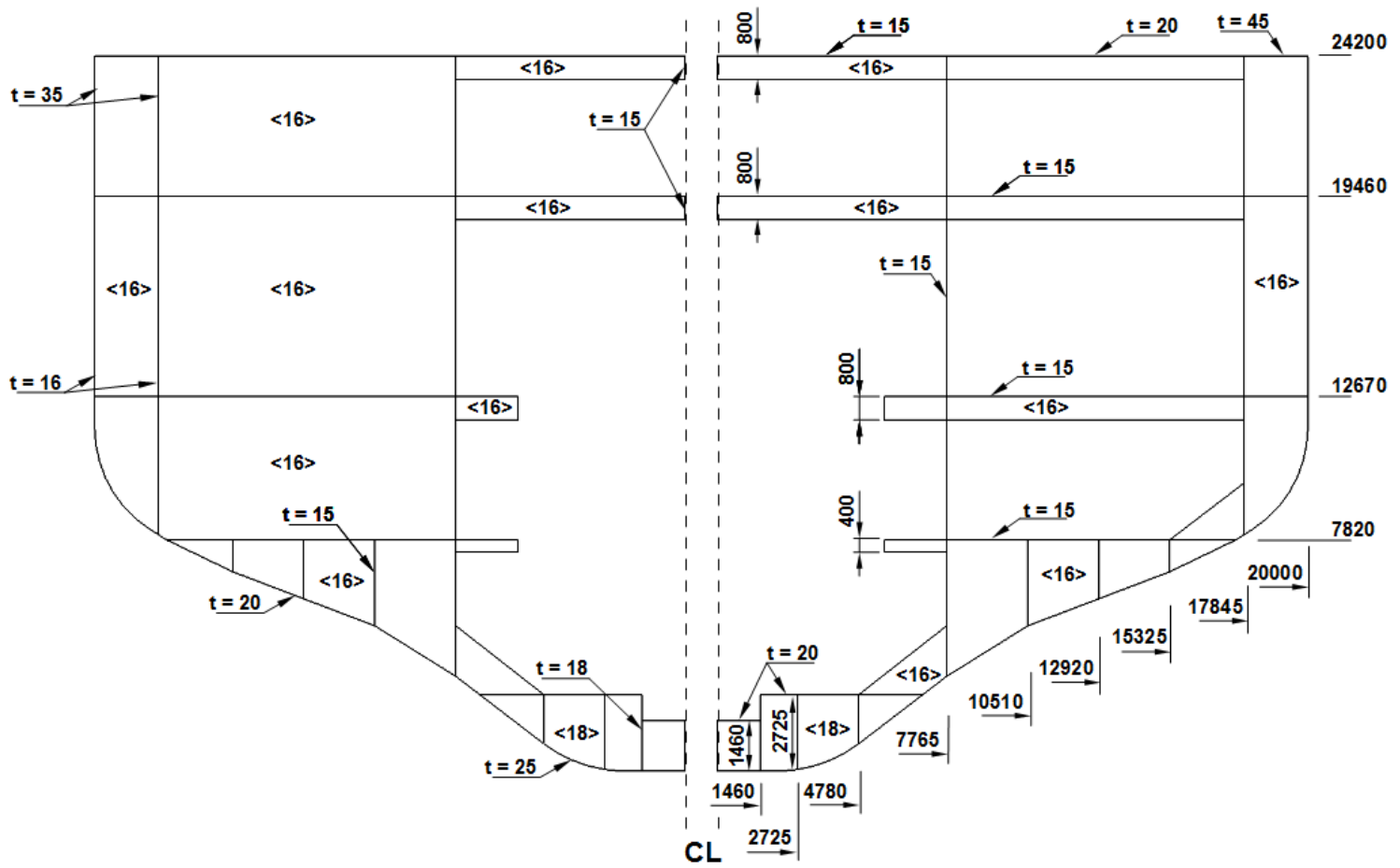

Figure C.4.: Cross section of simplified stern section. 


\section{C.2. Moment-shear force interaction of bow and stern sections}

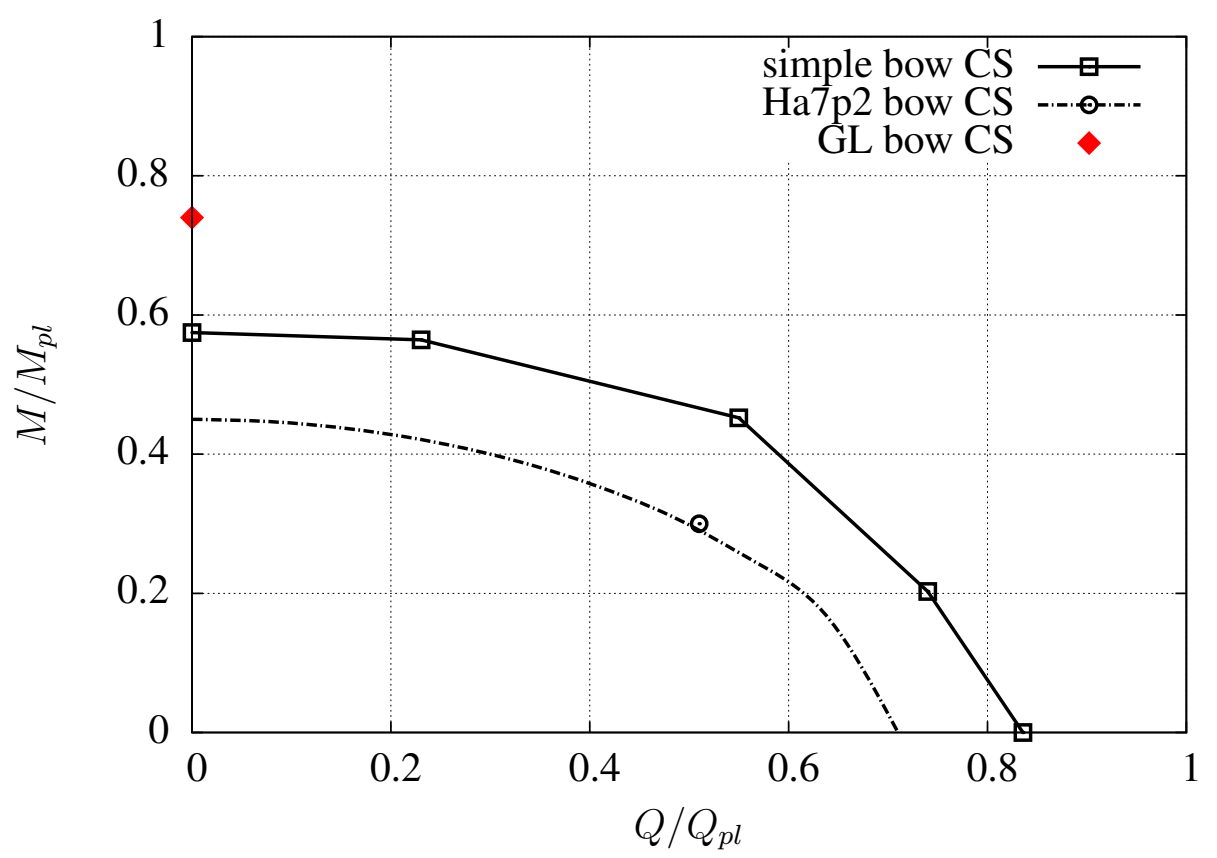

Figure C.5.: Bow section: moment-shear force interaction curve.

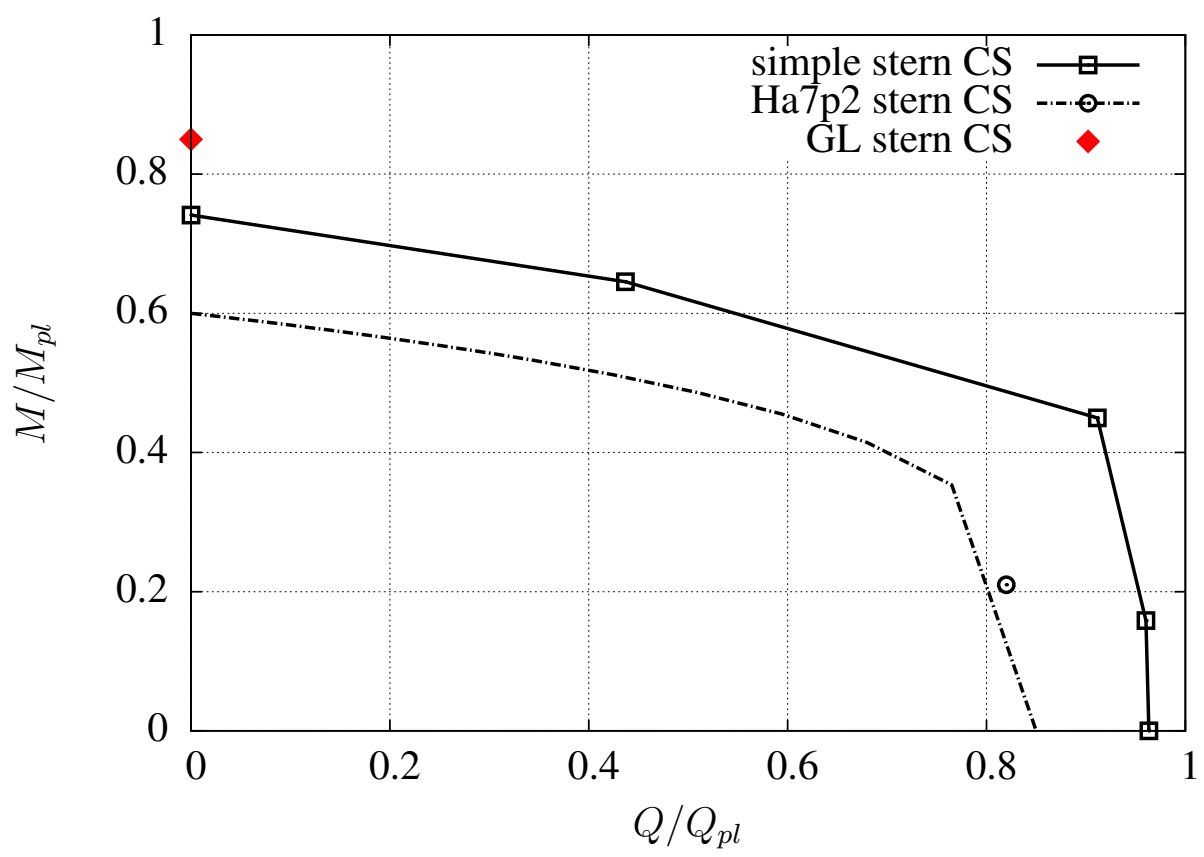

Figure C.6.: Stern sections: moment-shear force interaction curve. 
